\title{
Mechanistic Insight Towards Understanding the Role of Charge in Thiourea Organocatalysis
}

Ivor Smajlagic, ${ }^{\dagger}$ Matt Guest, ${ }^{\dagger}$ Rocio Durán, ${ }^{\ddagger}$ Barbara Herrera,${ }^{\ddagger}$ and Travis Dudding ${ }^{+*}$

†Department of Chemistry, Brock University, 1812 Sir Isaac Brock Way, St. Catharines, Ontario, Canada, L2S 3A1.

${ }^{\ddagger}$ Laboratorio de Química Teórica Computacional (QTC), Departamento de Química-Física, Facultad de Química y de Farmacia, Pontificia Universidad Católica de Chile, Av. Vicuña Mackenna 4860, Macul, Santiago, Chile.

Email: tdudding@brocku.ca

\section{SUPPORTING INFORMATION}

\section{Table of Contents:}

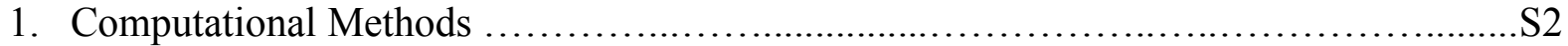

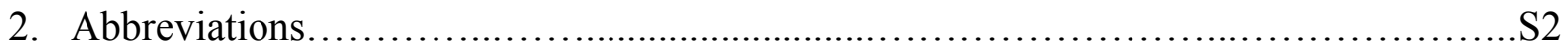

3. Thiourea Conformers, Methanol, DHP and MTP Optimized Structures ............ S3-S10

4. Pathway 1 (Dual Hydrogen Bond Donor-Catalyzed Mechanism).................. S11-S17

5. Pathway 2 (Brønsted Acid-Catalyzed Mechanism)............................ S18-S84

6. Natural Bond Order (NBO) Studies......................................... 84

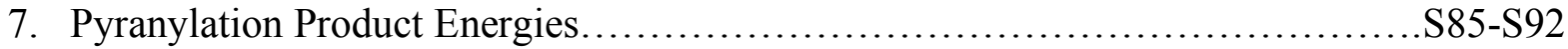

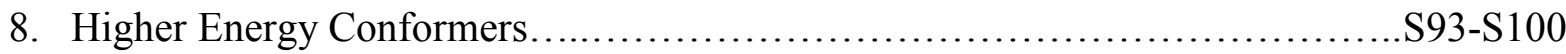

9. Rotational Energy Barriers Between $E, Z$ - and Z,Z-Conformers...............S101-S111

10. Conformational Search for $\alpha$ - vs. $\beta$-Stereofacial Alcohol Addition.................S112

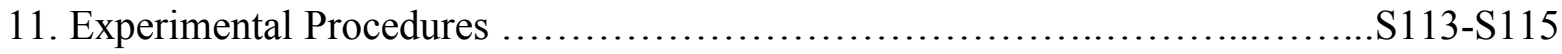

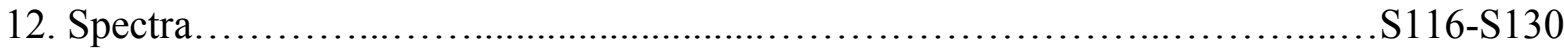

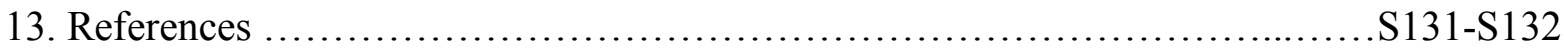




\section{Computational Methods}

All calculations were performed with the Gaussian $09^{1}$ suite of programs at the Density Functional Theory (DFT) level employing the range-separated hybrid $\omega$ B97X-D functional ${ }^{2}$ with a $6-311 \mathrm{G}(\mathrm{d}, \mathrm{p})$ basis set. ${ }^{3}$ This functional was selected as it accounts for dispersion and has been shown to provide accurate thermochemical and kinetic energies. Single-point

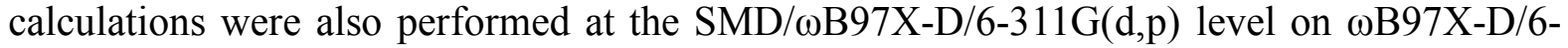
$311 \mathrm{G}(\mathrm{d}, \mathrm{p})$ optimized geometries. Thermal corrections from the vibrational frequencies at the $\omega \mathrm{B} 97 \mathrm{X}-\mathrm{D} / 6-311 \mathrm{G}(\mathrm{d}, \mathrm{p})$ were added to the electronic energies of the abovementioned methods to gain the free energies. All of the optimized geometries (minimum stationary points (reactants, intermediates and products)) were confirmed by frequency computations as minima (zero imaginary frequencies) or transition states (one imaginary frequency) using analytic second derivatives. ${ }^{4}$ Intrinsic reaction coordinate (IRC) calculations were performed to ensure that the transition states found were first order saddle points connecting the reactants and the products. ${ }^{5,6,7}$ Solvent dichloromethane (DCM $\left.-\varepsilon=8.93\right)$ in these calculations was accounted for by the Self-Consistent Reaction Field (SCRF) method using the Solvation Model based on Density (SMD) model to gain a more accurate treatment of medium/long ranged attractive noncovalent interactions. ${ }^{8,9}$ The Gibbs free energy differences $\left(\Delta G^{0}\right)$ were taken with respect to the most stable conformer. The 3D images of all optimized geometries were generated with CYLview $^{10}$. GaussView ${ }^{11}$ was used to construct all structures prior to geometrical optimization. Monte Carlo conformational searches (MCCS) with an OPLS3 ${ }^{12}$ force field were performed on the full catalyst systems using Macro-model program in Schrodinger. ${ }^{13}$

\section{Abbreviations}

INT - Intermediate

TS - Transition State

PA - Product Assisted

MA - Methanol Assisted

An - Anilino Protonation Pathway

Cy - Cyclopropenium Protonation Pathway 


\title{
3. Thiourea Conformers, Methanol, DHP and MTP Optimized Structures
}

\author{
a. Methanol, DHP and MTP Optimized Structures
}

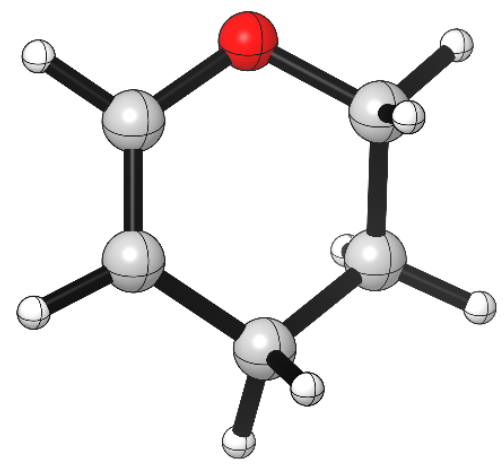

Figure S1. Optimized Structure of DHP.

- Thermochemistry -

(0 imaginary frequencies)

Zero-point correction $=$

Thermal correction to Energy=

0.123525 (Hartree/Particle)

Thermal correction to Enthalpy=

0.128718

0.129662

Thermal correction to Gibbs Free Energy=

0.095132

Sum of electronic and zero-point Energies=

$-270.403457$

Sum of electronic and thermal Energies=

$-270.398264$

Sum of electronic and thermal Enthalpies=

$-270.397320$

Sum of electronic and thermal Free Energies=

$-270.431850$

$\begin{array}{rrrc}\mathrm{H} & -0.64766000 & 2.41093800 & 0.09524600 \\ \mathrm{C} & 0.91359900 & 1.05500200 & -0.08177700 \\ \mathrm{C} & -0.37391700 & 1.36348800 & 0.06244200 \\ \mathrm{C} & -1.44414700 & 0.30830200 & 0.12582700 \\ \mathrm{C} & -0.86010300 & -1.03348500 & -0.32286700 \\ \mathrm{C} & 0.51897700 & -1.21851700 & 0.29381700 \\ \mathrm{H} & -1.84990000 & 0.22676900 & 1.14180100\end{array}$

$\mathrm{H} \quad \begin{array}{llll}-2.28438100 & 0.58826600 & -0.51726100\end{array}$

$\mathrm{H} \quad-1.50547500-1.86745600 \quad-0.03494100$

$\mathrm{H} \quad-0.76026900 \quad-1.05171300 \quad-1.41256200$

$\mathrm{H} \quad 0.44481200 \quad-1.19741800 \quad 1.38949700$

$\mathrm{H} \quad 0.97869200 \quad-2.15970500 \quad-0.00740900$

$\begin{array}{llll}\mathrm{O} & 1.42560700 & -0.19971800 & -0.11544900\end{array}$

$\begin{array}{llll}\mathrm{H} & -1.84990000 & 0.22676900 & 1.14180100\end{array}$

$\mathrm{H}$

$\begin{array}{llll}1.69287200 & 1.79932600 & -0.19543900\end{array}$ 


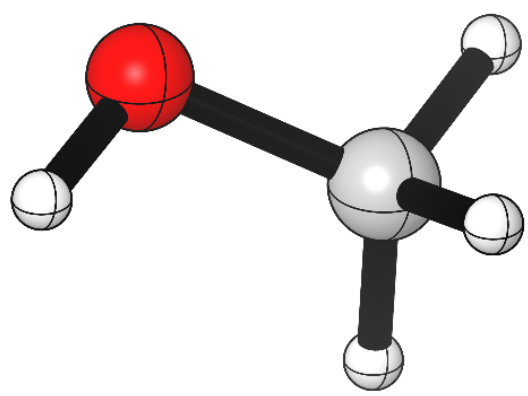

Figure S2. Optimized Structure of Methanol.

\section{- Thermochemistry -}

(0 imaginary frequencies)

Zero-point correction $=$ Thermal correction to Energy= 0.051766 (Hartree/Particle) Thermal correction to Enthalpy= 0.055076

Thermal correction to Gibbs Free Energy $=\quad 0.029039$

Sum of electronic and zero-point Energies $=\quad-115.665488$

Sum of electronic and thermal Energies=

$-115.662178$

Sum of electronic and thermal Enthalpies=

$-115.661234$

Sum of electronic and thermal Free Energies=

$-115.688215$

$\begin{array}{cccccrcc}\mathrm{C} & 0.65848200 & -0.01957300 & 0.00000800 & \mathrm{H} & 1.08204600 & 0.98553300 & 0.00113000 \\ \mathrm{H} & 1.02945200 & -0.54148000 & -0.89234200 & \mathrm{O} & -0.74534100 & 0.12146400 & 0.00001600 \\ \mathrm{H} & 1.02937400 & -0.54356800 & 0.89115600 & \mathrm{H} & -1.12903900 & -0.75476300 & -0.00011900\end{array}$

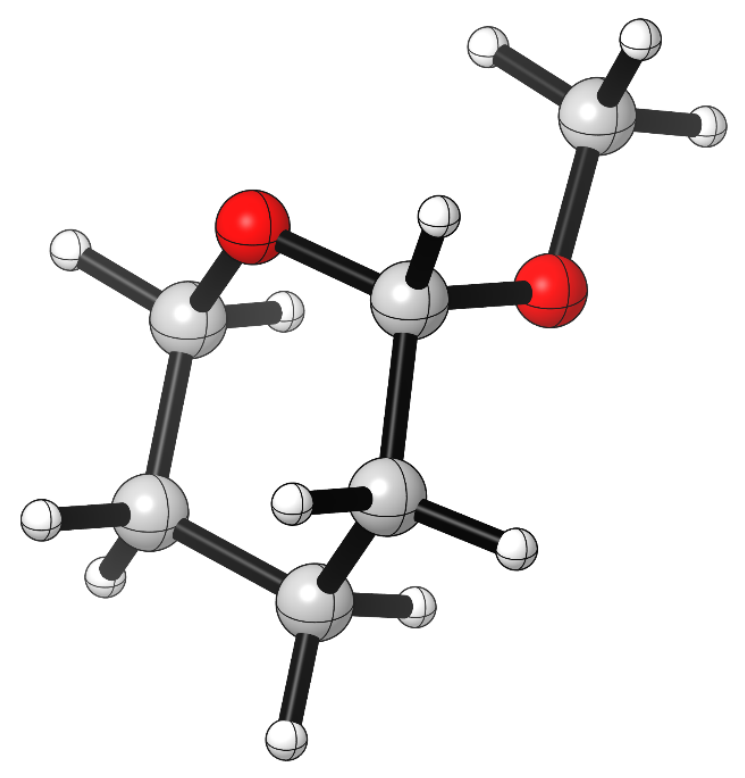


Figure S3. Optimized Structure of MTP.

- Thermochemistry -

(0 imaginary frequencies)

Zero-point correction $=$

Thermal correction to Energy=

0.180759 (Hartree/Particle)

Thermal correction to Enthalpy=

0.188582

Thermal correction to Gibbs Free Energy=

0.189526

Sum of electronic and zero-point Energies=

0.148695

Sum of electronic and thermal Energies $=$

$-386.104437$

$-386.096615$

Sum of electronic and thermal Enthalpies=

$-386.095670$

Sum of electronic and thermal Free Energies=

$-386.136501$

$\begin{array}{rrrrrrrr}\mathrm{H} & -1.12543900 & -1.45597400 & 1.44154200 & \mathrm{H} & -0.17049200 & 1.48610700 & -1.05994100 \\ \mathrm{C} & 0.56123200 & -0.42583800 & 0.65141900 & \mathrm{H} & -0.95875900 & 2.43336700 & 0.21654500 \\ \mathrm{C} & -0.61112100 & -1.38264500 & 0.47788300 & \mathrm{O} & 0.13807400 & 0.89364700 & 0.90044400 \\ \mathrm{C} & -1.57807300 & -0.87401100 & -0.59187500 & \mathrm{H} & 1.15984700 & -0.68535700 & 1.53453400 \\ \mathrm{C} & -1.96737700 & 0.57307400 & -0.28584000 & \mathrm{H} & -0.22107200 & -2.37168700 & 0.22820200 \\ \mathrm{C} & -0.71565000 & 1.42114000 & -0.10893100 & \mathrm{C} & 2.55432100 & 0.21829500 & -0.41977700 \\ \mathrm{H} & -1.08956000 & -0.92351500 & -1.56998500 & \mathrm{H} & 3.17600500 & -0.13813300 & 0.41320900 \\ \mathrm{H} & -2.46352900 & -1.51358700 & -0.63682200 & \mathrm{H} & 2.36634800 & 1.28794000 & -0.28167800 \\ \mathrm{H} & -2.58287100 & 0.99284100 & -1.08724700 & \mathrm{H} & 3.08788800 & 0.05930000 & -1.35638400 \\ \mathrm{H} & -2.55599600 & 0.61161200 & 0.63782900 & \mathrm{O} & 1.35163200 & -0.51402200 & -0.50257900\end{array}$

\section{b. Thiourea Conformers}

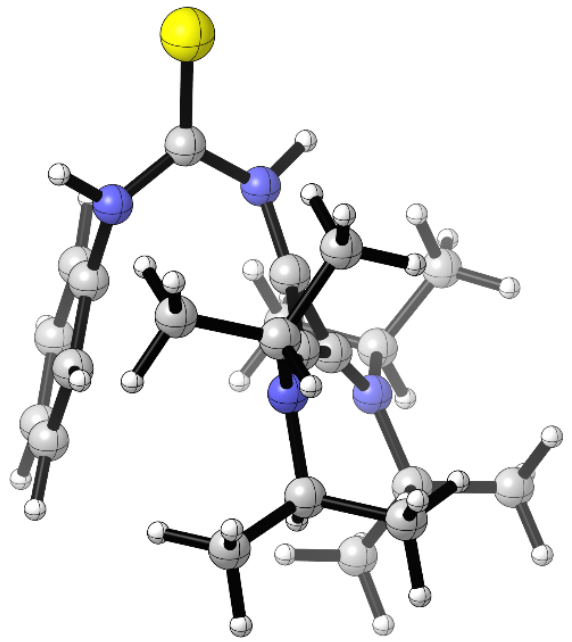

Figure S4. Optimized Structure of Thiourea $E, E-1$ Conformer.

- Thermochemistry - 
(0 imaginary frequencies)

Zero-point correction $=$

Thermal correction to Energy=

Thermal correction to Enthalpy=

Thermal correction to Gibbs Free Energy=

Sum of electronic and zero-point Energies=

Sum of electronic and thermal Energies=

Sum of electronic and thermal Enthalpies=

Sum of electronic and thermal Free Energies=

\subsection{4 (Hartree/Particle)}

0.578341

0.579285

0.488250

$-1475.778161$

$-1475.748223$

$-1475.747279$

$-1475.838315$

$\begin{array}{lrrr}\mathrm{C} & -2.67507200 & -0.90203300 & -1.00755200 \\ \mathrm{~S} & -3.91896400 & -1.04556400 & -2.07371300 \\ \mathrm{~N} & -1.34767200 & -1.08138300 & -1.41388600 \\ \mathrm{H} & -1.23730600 & -1.54782700 & -2.30246100 \\ \mathrm{~N} & -2.81900800 & -0.60337800 & 0.31032700 \\ \mathrm{C} & -1.94325200 & -1.04565800 & 1.35934100 \\ \mathrm{C} & -1.63223700 & -2.39601200 & 1.47994200 \\ \mathrm{C} & -1.44525200 & -0.12513000 & 2.27477700 \\ \mathrm{C} & -0.80043600 & -2.81980600 & 2.50740400 \\ \mathrm{H} & -2.05036700 & -3.10776900 & 0.77690300 \\ \mathrm{C} & -0.61691100 & -0.55613800 & 3.30308900 \\ \mathrm{H} & -1.70749900 & 0.92049000 & 2.17758400 \\ \mathrm{C} & -0.28809200 & -1.90205300 & 3.41713000 \\ \mathrm{H} & 0.35092400 & -2.23828800 & 4.22498900 \\ \mathrm{H} & -3.78106200 & -0.43535200 & 0.57130100 \\ \mathrm{H} & -0.23905900 & 0.15916600 & 4.02441400 \\ \mathrm{H} & -0.56661300 & -3.87316400 & 2.60714300 \\ \mathrm{C} & -0.31516200 & -0.32929500 & -0.94774000 \\ \mathrm{C} & 0.15094000 & 0.85577200 & -0.43761600 \\ \mathrm{C} & 1.02292000 & -0.23408900 & -0.65485700 \\ \mathrm{~N} & -0.02814100 & 2.09222700 & -0.03073300 \\ \mathrm{~N} & 2.21985400 & -0.76353800 & -0.64376300 \\ \mathrm{C} & 1.07403300 & 2.76115300 & 0.70053800 \\ \mathrm{C} & -1.15988000 & 2.91153600 & -0.54970300 \\ \mathrm{C} & 3.39997000 & 0.03981100 & -0.25429400 \\ \mathrm{C} & 2.38492900 & -2.18931800 & -1.01966900 \\ \mathrm{C} & 1.93430200 & 3.60406200 & -0.23788800 \\ \mathrm{H} & 1.67807300 & 1.94612100 & 1.10620100 \\ \mathrm{C} & 0.54976800 & 3.55816900 & 1.88973500 \\ \mathrm{C} & -2.40041100 & 2.84264100 & 0.33216900 \\ \mathrm{H} & -0.77738900 & 3.93415200 & -0.52526800\end{array}$

$\begin{array}{lrrr}\mathrm{C} & -1.47358200 & 2.57504100 & -2.00373200 \\ \mathrm{H} & 3.02679600 & 1.05721800 & -0.13664100 \\ \mathrm{C} & 3.94994400 & -0.43352400 & 1.08755900 \\ \mathrm{C} & 4.45016800 & 0.06379300 & -1.36017300 \\ \mathrm{H} & 3.43145100 & -2.41666100 & -0.81292200 \\ \mathrm{C} & 1.51987500 & -3.08989000 & -0.14783400 \\ \mathrm{C} & 2.13483400 & -2.39060100 & -2.51076800 \\ \mathrm{H} & 2.28902400 & 3.01577000 & -1.08854100 \\ \mathrm{H} & 1.38098000 & 4.46156400 & -0.62830400 \\ \mathrm{H} & 2.80374300 & 3.99163500 & 0.29742900 \\ \mathrm{H} & -0.06609500 & 4.40456400 & 1.57724900 \\ \mathrm{H} & -0.03734100 & 2.92517100 & 2.55797200 \\ \mathrm{H} & 1.39443900 & 3.95885800 & 2.45316500 \\ \mathrm{H} & -2.83779900 & 1.84404200 & 0.29844700 \\ \mathrm{H} & -2.17681100 & 3.09984700 & 1.36868800 \\ \mathrm{H} & -3.14595100 & 3.55069000 & -0.03577500 \\ \mathrm{H} & -0.57361300 & 2.60474800 & -2.62261600 \\ \mathrm{H} & -1.94712400 & 1.59525000 & -2.10794100 \\ \mathrm{H} & -2.17815900 & 3.30988000 & -2.39659300 \\ \mathrm{H} & 4.34903600 & -1.44908700 & 1.02244900 \\ \mathrm{H} & 4.76506900 & 0.21909900 & 1.40683400 \\ \mathrm{H} & 3.17200400 & -0.41785200 & 1.85469500 \\ \mathrm{H} & 4.02173400 & 0.41564700 & -2.30101200 \\ \mathrm{H} & 5.25624700 & 0.74358500 & -1.07734000 \\ \mathrm{H} & 4.89668600 & -0.91955500 & -1.52595400 \\ \mathrm{H} & 1.74670200 & -4.13687900 & -0.35822800 \\ \mathrm{H} & 1.69980900 & -2.90073500 & 0.91218700 \\ \mathrm{H} & 0.45623400 & -2.93260700 & -0.34418000 \\ \mathrm{H} & 1.09453100 & -2.17137200 & -2.76948100 \\ \mathrm{H} & 2.78084200 & -1.74988500 & -3.11314000 \\ \mathrm{H} & 2.32767900 & -3.42951400 & -2.78460100\end{array}$




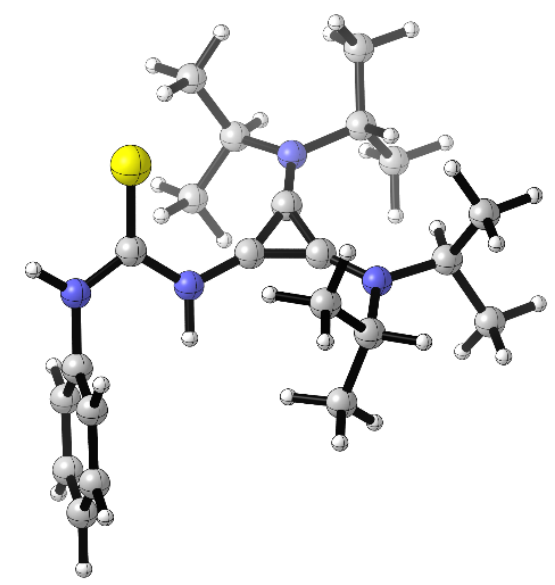

Figure S5. Optimized Structure of Thiourea E,Z-1 Conformer.

- Thermochemistry -

(0 imaginary frequencies)

Zero-point correction $=$

Thermal correction to Energy=

Thermal correction to Enthalpy=

Thermal correction to Gibbs Free Energy=

Sum of electronic and zero-point Energies=

Sum of electronic and thermal Energies=

Sum of electronic and thermal Enthalpies=

Sum of electronic and thermal Free Energies=
0.547610 (Hartree/Particle)

0.578095

0.579039

0.484071

$-1475.780215$

$-1475.749730$

$-1475.748786$

$-1475.843754$

$\begin{array}{lrrr}\mathrm{C} & 2.67232200 & 2.64355700 & 0.14263800 \\ \mathrm{C} & 0.24591000 & 3.05570200 & -0.58981300 \\ \mathrm{C} & 4.20023400 & -0.51277400 & 1.78620400 \\ \mathrm{H} & 3.76685300 & 0.04155600 & -0.24520000 \\ \mathrm{C} & 4.75281900 & -1.82825300 & -0.31206900 \\ \mathrm{C} & 2.02712100 & -3.75699900 & -0.32879100 \\ \mathrm{H} & 2.94578000 & -2.99548200 & 1.45353800 \\ \mathrm{C} & 0.84136500 & -2.78757400 & 1.68213600 \\ \mathrm{H} & 3.21072600 & 1.80220800 & 0.57963300 \\ \mathrm{C} & 3.37503300 & 3.06260500 & -1.14420000 \\ \mathrm{C} & 2.63687700 & 3.75051400 & 1.19199100 \\ \mathrm{H} & 0.74685700 & 4.01238900 & -0.74337200 \\ \mathrm{C} & -0.34237800 & 2.61496900 & -1.92669900 \\ \mathrm{C} & -0.81253100 & 3.24033200 & 0.49378200 \\ \mathrm{H} & 3.49906500 & 0.19105600 & 2.24337400 \\ \mathrm{H} & 4.24810800 & -1.40222200 & 2.41948100 \\ \mathrm{H} & 5.19209100 & -0.05567000 & 1.78364100 \\ \mathrm{H} & 4.84382300 & -2.77201500 & 0.22992100 \\ \mathrm{H} & 4.45307100 & -2.03854500 & -1.33971500 \\ \mathrm{H} & 5.74201300 & -1.36691600 & -0.33453500 \\ \mathrm{H} & 1.18746500 & -3.52801300 & -0.98533000 \\ \mathrm{H} & 2.94219500 & -3.76555300 & -0.92034500 \\ \mathrm{H} & 1.88248200 & -4.75819500 & 0.08391900 \\ \mathrm{H} & 0.87739800 & -2.03274500 & 2.47175400\end{array}$




$\begin{array}{rrrrrrrr}\mathrm{H} & -0.06488500 & -2.64533000 & 1.08891900 & \mathrm{H} & 2.12892900 & 4.64706000 & 0.82847900 \\ \mathrm{H} & 0.76775600 & -3.76820300 & 2.15499400 & \mathrm{H} & -1.04729000 & 3.36882300 & -2.28253200 \\ \mathrm{H} & 2.87249600 & 3.91059600 & -1.61720000 & \mathrm{H} & 0.43955900 & 2.49494100 & -2.67890900 \\ \mathrm{H} & 4.40080100 & 3.36785300 & -0.92776200 & \mathrm{H} & -0.88406700 & 1.66911000 & -1.84116800 \\ \mathrm{H} & 3.40424600 & 2.23914600 & -1.86168500 & \mathrm{H} & -1.34962200 & 2.30764600 & 0.68963600 \\ \mathrm{H} & 2.14020000 & 3.41261400 & 2.10386600 & \mathrm{H} & -0.36664500 & 3.58111100 & 1.42955600 \\ \mathrm{H} & 3.65898300 & 4.03708300 & 1.44683900 & \mathrm{H} & -1.54964000 & 3.97932500 & 0.17420200\end{array}$

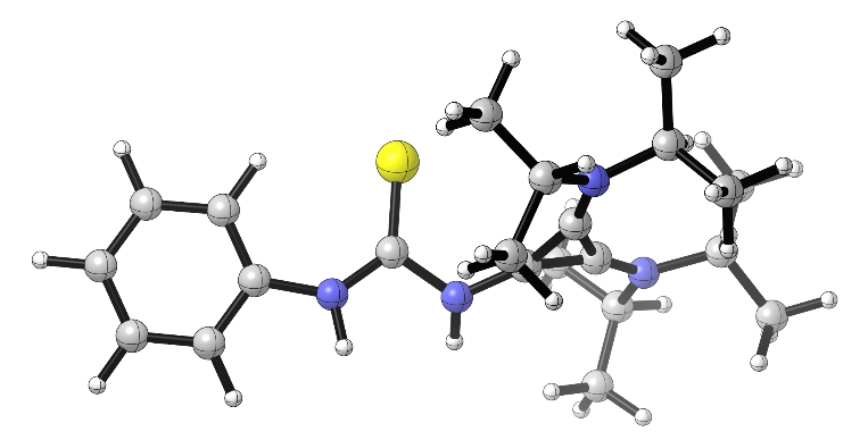

Figure S6. Optimized Structure of Thiourea Z,Z-1 Conformer.

- Thermochemistry -

(0 imaginary frequencies)

Zero-point correction=

Thermal correction to Energy=

Thermal correction to Enthalpy=

Thermal correction to Gibbs Free Energy=

Sum of electronic and zero-point Energies=

Sum of electronic and thermal Energies $=$

Sum of electronic and thermal Enthalpies=

Sum of electronic and thermal Free Energies=
0.547201 (Hartree/Particle)

$$
0.577826
$$

0.578770

$$
\begin{gathered}
0.483301 \\
-1475.771861 \\
-1475.741236 \\
-1475.740292 \\
-1475.835761
\end{gathered}
$$

$\begin{array}{lrrr}\mathrm{C} & 1.86398800 & -0.31005200 & 0.05797100 \\ \mathrm{~S} & 1.62562600 & 0.30685800 & 1.56955500 \\ \mathrm{~N} & 0.76360300 & -0.76800100 & -0.72160900 \\ \mathrm{H} & 0.84943900 & -1.70703900 & -1.08799000 \\ \mathrm{~N} & 3.00543700 & -0.48496400 & -0.62887500 \\ \mathrm{C} & 4.37426500 & -0.38255800 & -0.26307100 \\ \mathrm{C} & 4.83592200 & 0.17170800 & 0.92696400 \\ \mathrm{C} & 5.28314800 & -0.87334700 & -1.20404000 \\ \mathrm{C} & 6.20563300 & 0.21929900 & 1.16246900 \\ \mathrm{H} & 4.14417900 & 0.56412100 & 1.65472800 \\ \mathrm{C} & 6.64338200 & -0.81272500 & -0.95769400 \\ \mathrm{H} & 4.92393900 & -1.30941400 & -2.13178800 \\ \mathrm{C} & 7.11176200 & -0.26654100 & 0.23220200 \\ \mathrm{H} & 8.17586000 & -0.21762200 & 0.42785600 \\ \mathrm{H} & 2.87654600 & -0.74291900 & -1.59737600 \\ \mathrm{H} & 6.56122400 & 0.65154600 & 2.09011000 \\ \mathrm{H} & 7.33822000 & -1.19496500 & -1.69558200\end{array}$

$\begin{array}{lrrr}\mathrm{C} & -0.50524000 & -0.37087900 & -0.43124600 \\ \mathrm{C} & -1.80755500 & -0.70717900 & -0.17843000 \\ \mathrm{C} & -1.40996300 & 0.64604200 & -0.25448800 \\ \mathrm{~N} & -2.72122900 & -1.63271700 & -0.00505100 \\ \mathrm{~N} & -1.72956400 & 1.91392500 & -0.18051900 \\ \mathrm{C} & -4.15346600 & -1.27767200 & 0.10001600 \\ \mathrm{C} & -2.28481500 & -3.03873800 & 0.16837700 \\ \mathrm{C} & -3.10916400 & 2.27581900 & 0.22813100 \\ \mathrm{C} & -0.81938100 & 2.96680400 & -0.70787900 \\ \mathrm{C} & -4.99892900 & -2.08580300 & -0.87969500 \\ \mathrm{H} & -4.21357500 & -0.23285700 & -0.20855400 \\ \mathrm{C} & -4.63515700 & -1.39395100 & 1.54262400 \\ \mathrm{C} & -1.27494100 & -3.16773500 & 1.30461000 \\ \mathrm{H} & -3.18480500 & -3.58249000 & 0.45831000 \\ \mathrm{C} & -1.78191400 & -3.61950900 & -1.14979100 \\ \mathrm{H} & -3.48063000 & 1.40620700 & 0.77426900 \\ \mathrm{C} & -3.12798000 & 3.44982600 & 1.19985300\end{array}$




$\begin{array}{lrrrrrrr}\mathrm{C} & -3.99060800 & 2.51089200 & -0.99581100 & \mathrm{H} & -0.89417900 & -3.08758000 & -1.50631900 \\ \mathrm{H} & -1.48917000 & 3.77412000 & -1.01271500 & \mathrm{H} & -1.50832800 & -4.66805500 & -1.01861100 \\ \mathrm{C} & 0.13543600 & 3.49540200 & 0.35674300 & \mathrm{H} & -2.77688600 & 4.37448600 & 0.73643200 \\ \mathrm{C} & -0.07959500 & 2.49157300 & -1.95438900 & \mathrm{H} & -4.15488000 & 3.61899000 & 1.52942500 \\ \mathrm{H} & -4.62834100 & -1.97532100 & -1.90089800 & \mathrm{H} & -2.51703100 & 3.24284200 & 2.07988400 \\ \mathrm{H} & -5.01983500 & -3.14902400 & -0.62867300 & \mathrm{H} & -3.97558300 & 1.64805100 & -1.66750200 \\ \mathrm{H} & -6.02879100 & -1.72495500 & -0.84963300 & \mathrm{H} & -5.02324500 & 2.68764500 & -0.68738400 \\ \mathrm{H} & -4.57949100 & -2.42572800 & 1.89992500 & \mathrm{H} & -3.66184100 & 3.38620300 & -1.56178600 \\ \mathrm{H} & -4.03295200 & -0.76999100 & 2.20760400 & \mathrm{H} & 0.71626100 & 4.32325600 & -0.05646800 \\ \mathrm{H} & -5.67667700 & -1.07499100 & 1.61863500 & \mathrm{H} & -0.39823100 & 3.86119200 & 1.23398300 \\ \mathrm{H} & -0.33340000 & -2.65919300 & 1.07894500 & \mathrm{H} & 0.82758400 & 2.71452100 & 0.67585500 \\ \mathrm{H} & -1.67237300 & -2.74369900 & 2.22869500 & \mathrm{H} & 0.67779100 & 1.74140200 & -1.71440200 \\ \mathrm{H} & -1.05010200 & -4.22151700 & 1.47941200 & \mathrm{H} & -0.76822800 & 2.07833500 & -2.69547000 \\ \mathrm{H} & -2.54531100 & -3.55708300 & -1.92694300 & \mathrm{H} & 0.43613000 & 3.34004300 & -2.40703800\end{array}$

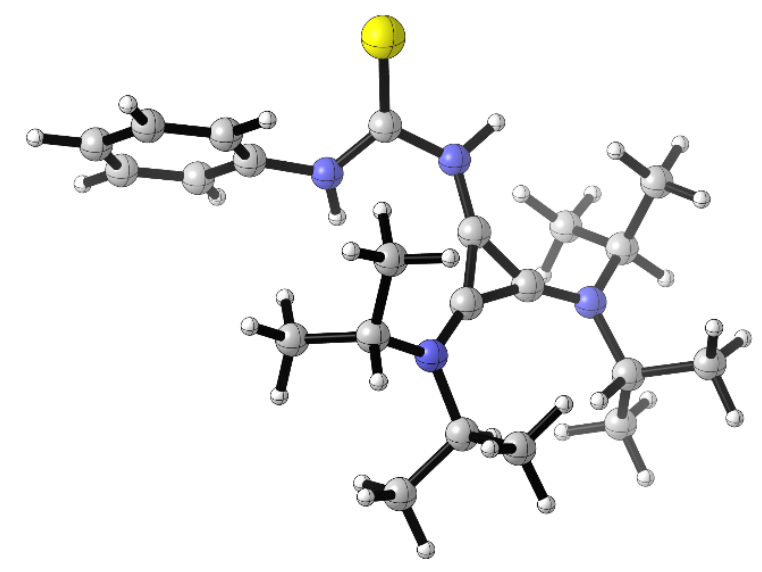

Figure S7. Optimized Structure of Thiourea Z,E-1 Conformer.

- Thermochemistry -

(0 imaginary frequencies)

Zero-point correction $=$

Thermal correction to Energy=

Thermal correction to Enthalpy=

Thermal correction to Gibbs Free Energy=
Sum of electronic and zero-point Energies

Sum of electronic and thermal Energies=

Sum of electronic and thermal Enthalpies=

Sum of electronic and thermal Free Energies=

\subsection{1 (Hartree/Particle)}

0.578206

0.579150

0.486208

$-1475.771922$

$-1475.741657$

$-1475.740712$

$-1475.833654$
$\begin{array}{lrrr}\mathrm{C} & -1.63146500 & -1.76941200 & -0.42887300 \\ \mathrm{~S} & -2.43186200 & -2.79788500 & -1.42558100 \\ \mathrm{~N} & -0.22422200 & -1.81905600 & -0.35961700 \\ \mathrm{H} & 0.16714300 & -2.66589200 & -0.74736500 \\ \mathrm{~N} & -2.14163400 & -0.84144700 & 0.41110000 \\ \mathrm{C} & -3.50495600 & -0.44938500 & 0.53280800\end{array}$

$\begin{array}{lrrr}\text { C } & -4.25399200 & -0.10000800 & -0.58550700 \\ \mathrm{C} & -4.04658900 & -0.34179100 & 1.80817600 \\ \mathrm{C} & -5.55680500 & 0.34443200 & -0.41741800 \\ \mathrm{H} & -3.82742500 & -0.18499300 & -1.57602700 \\ \mathrm{C} & -5.34743400 & 0.11741900 & 1.96679000 \\ \mathrm{H} & -3.45816300 & -0.62691200 & 2.67423800\end{array}$




$\begin{array}{lrrrrrrr}\mathrm{C} & -6.10605500 & 0.45780800 & 0.85475400 & \mathrm{C} & 3.32183900 & -3.07460300 & -0.71521000 \\ \mathrm{H} & -7.12346800 & 0.80832700 & 0.97827100 & \mathrm{H} & 2.85801100 & 2.33133300 & -1.74409700 \\ \mathrm{H} & -1.54371900 & -0.55189500 & 1.17180300 & \mathrm{H} & 1.86836400 & 3.79222500 & -1.86416700 \\ \mathrm{H} & -6.14535100 & 0.60690400 & -1.28833900 & \mathrm{H} & 3.36896300 & 3.82602200 & -0.94123200 \\ \mathrm{H} & -5.77014000 & 0.19849800 & 2.96082700 & \mathrm{H} & 0.57559600 & 4.63590300 & 0.27105700 \\ \mathrm{C} & 0.62113500 & -0.78081900 & -0.17271000 & \mathrm{H} & 0.74394600 & 3.70569500 & 1.77495500 \\ \mathrm{C} & 0.95102900 & 0.55925200 & -0.15213300 & \mathrm{H} & 2.10650700 & 4.63292400 & 1.13751400 \\ \mathrm{C} & 1.92846100 & -0.42812900 & 0.06211600 & \mathrm{H} & -1.99251000 & 1.67019300 & 0.59707700 \\ \mathrm{~N} & 0.66910000 & 1.84347100 & -0.23602200 & \mathrm{H} & -1.39632900 & 3.31423500 & 0.86623500 \\ \mathrm{~N} & 3.16683400 & -0.80708200 & 0.28305000 & \mathrm{H} & -2.56705200 & 2.98511600 & -0.41457900 \\ \mathrm{C} & 1.75587500 & 2.80682400 & 0.07440100 & \mathrm{H} & -0.16377600 & 1.28288200 & -2.75286200 \\ \mathrm{C} & -0.56677600 & 2.34443100 & -0.89725200 & \mathrm{H} & -1.37371300 & 0.49046800 & -1.71950300 \\ \mathrm{C} & 4.27855000 & 0.17022000 & 0.27901100 & \mathrm{H} & -1.79615100 & 1.94363100 & -2.60727800 \\ \mathrm{C} & 3.44410300 & -2.23866700 & 0.55545700 & \mathrm{H} & 5.25131500 & -0.51230400 & 2.10476800 \\ \mathrm{C} & 2.50464300 & 3.20815100 & -1.19467900 & \mathrm{H} & 5.58353800 & 1.16510100 & 1.68018500 \\ \mathrm{H} & 2.43437800 & 2.26369000 & 0.73559300 & \mathrm{H} & 4.00981000 & 0.72397200 & 2.36290200 \\ \mathrm{C} & 1.25237200 & 4.01236500 & 0.85943600 & \mathrm{H} & 4.95996500 & -0.36950100 & -1.71167000 \\ \mathrm{C} & -1.68985600 & 2.59272400 & 0.10408800 & \mathrm{H} & 6.12447100 & 0.55445900 & -0.75261100 \\ \mathrm{H} & -0.27096500 & 3.30585000 & -1.32322400 & \mathrm{H} & 5.87677000 & -1.15335600 & -0.41007600 \\ \mathrm{C} & -0.99011600 & 1.45323700 & -2.05895400 & \mathrm{H} & 2.90767000 & -3.77211600 & 1.96785800 \\ \mathrm{H} & 3.84317300 & 1.09986800 & -0.08608200 & \mathrm{H} & 2.69945700 & -2.12968400 & 2.59015700 \\ \mathrm{C} & 4.80756900 & 0.39713100 & 1.69137100 & \mathrm{H} & 1.52962400 & -2.81195900 & 1.44357200 \\ \mathrm{C} & 5.36962000 & -0.23281100 & -0.70874300 & \mathrm{H} & 2.31098000 & -3.03151500 & -1.13156100 \\ \mathrm{H} & 4.48499900 & -2.26661000 & 0.88088200 & \mathrm{H} & 4.01265500 & -2.72678600 & -1.48459700 \\ \mathrm{C} & 2.58977700 & -2.76127400 & 1.70609600 & \mathrm{H} & 3.54510200 & -4.12136800 & -0.50104400\end{array}$




\section{Pathway 1 (Dual Hydrogen Bond Donor-Catalyzed Mechanism)}

Scheme S1. Pathway 1: Dual Hydrogen Bond Activation Featuring Conformer Z,Z-1.

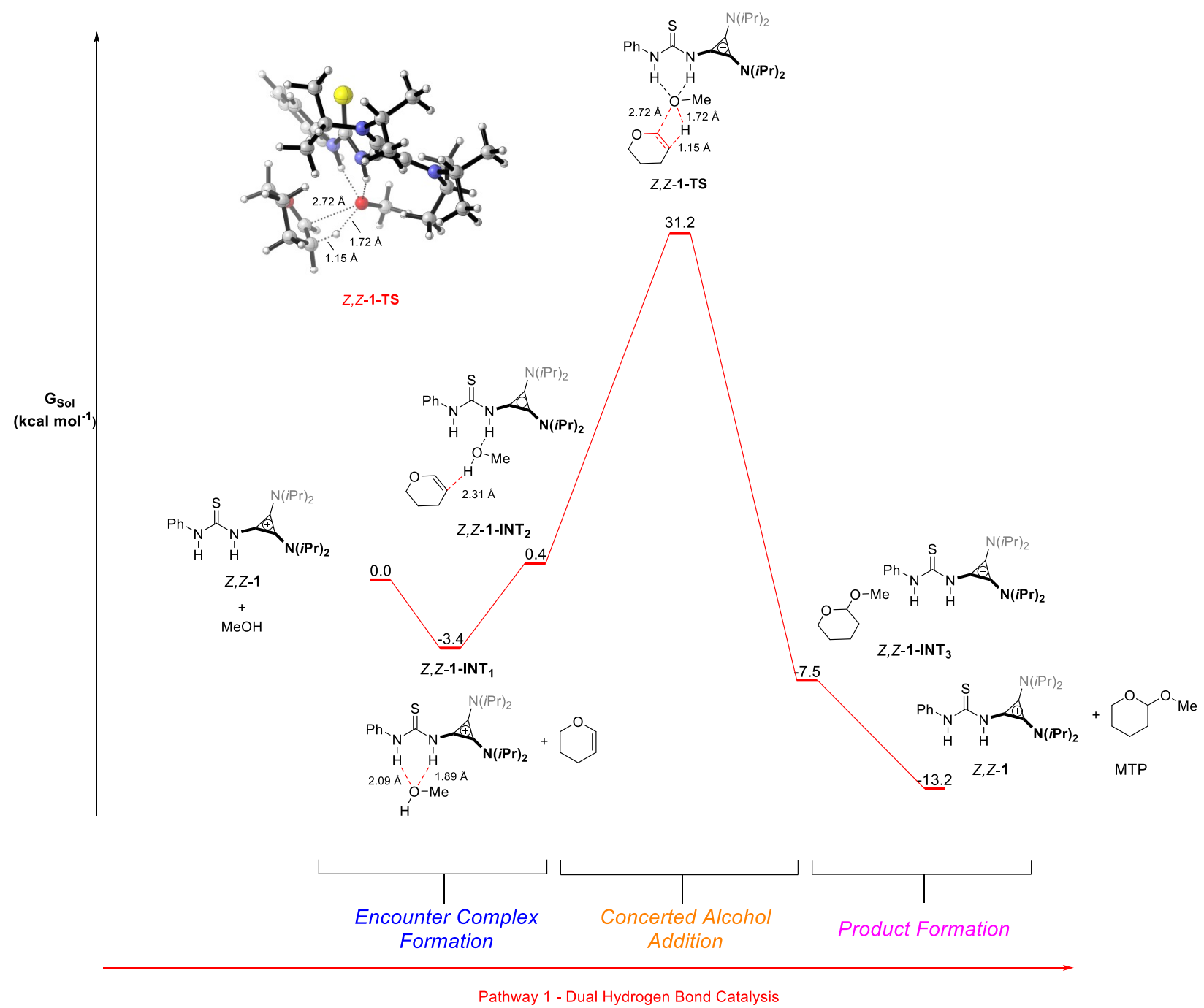




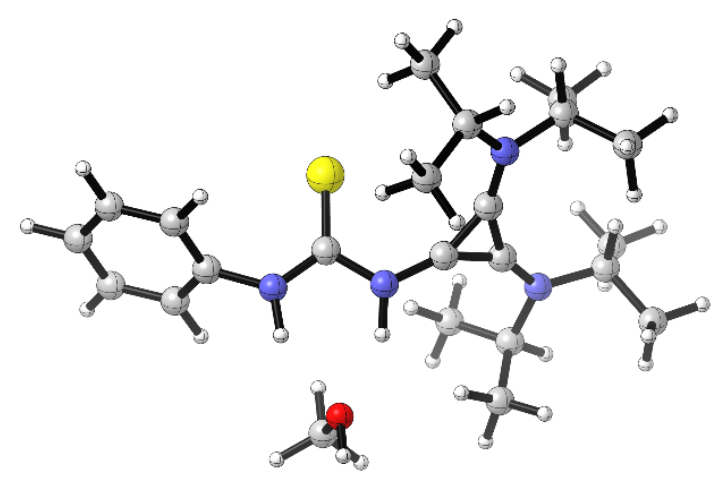

Figure S8. Optimized Structure of $Z, Z-1-$ INT $_{1}$.

- Thermochemistry -

\section{(0 imaginary frequencies)}

Zero-point correction=

Thermal correction to Energy=

Thermal correction to Enthalpy=

Thermal correction to Gibbs Free Energy=

Sum of electronic and zero-point Energies=

Sum of electronic and thermal Energies=

Sum of electronic and thermal Enthalpies=

Sum of electronic and thermal Free Energies=
0.602340 (Hartree/Particle)

0.637014

0.637958

0.534908

$-1591.465376$

$-1591.430702$

$-1591.429758$

$-1591.532808$

$\begin{array}{lrrr}\mathrm{C} & 1.69603800 & -0.20427400 & 0.12460100 \\ \mathrm{~S} & 1.49794700 & -1.23057500 & -1.16348200 \\ \mathrm{~N} & 2.86733700 & 0.23386100 & 0.62066600 \\ \mathrm{H} & 2.83053100 & 0.93058900 & 1.35682800 \\ \mathrm{~N} & 0.60924300 & 0.34511600 & 0.80579100 \\ \mathrm{H} & 0.77172800 & 1.19848800 & 1.33993900 \\ \mathrm{O} & 1.81316400 & 2.64542400 & 1.96899100 \\ \mathrm{H} & 1.85734100 & 3.03542800 & 2.84329900 \\ \mathrm{C} & 2.11991900 & 3.63202200 & 0.98271400 \\ \mathrm{H} & 3.12323600 & 4.03906100 & 1.12968500 \\ \mathrm{H} & 2.07899900 & 3.12766100 & 0.01670500 \\ \mathrm{H} & 1.38581300 & 4.44186100 & 0.99132100 \\ \mathrm{C} & 4.15111600 & -0.03909400 & 0.05444900 \\ \mathrm{C} & 4.71713100 & -1.30140900 & 0.17299700 \\ \mathrm{C} & 4.83810400 & 0.98977000 & -0.57658900 \\ \mathrm{C} & 5.97958700 & -1.53310600 & -0.35415300 \\ \mathrm{H} & 4.16582800 & -2.09218400 & 0.66641000 \\ \mathrm{C} & 6.10537100 & 0.75481800 & -1.09352000 \\ \mathrm{H} & 4.37552200 & 1.96596800 & -0.67222200 \\ \mathrm{C} & 6.67484400 & -0.50722800 & -0.98435600 \\ \mathrm{H} & 7.66095800 & -0.69257500 & -1.39303300 \\ \mathrm{H} & 6.42443400 & -2.51713800 & -0.26824500 \\ \mathrm{H} & 6.64274300 & 1.55527600 & -1.58775800 \\ \mathrm{C} & -0.67446200 & 0.01684100 & 0.49476200 \\ \mathrm{C} & -1.65736900 & -0.92935100 & 0.33358900 \\ \mathrm{C} & -1.90724500 & 0.45131600 & 0.09803600 \\ \mathrm{~N} & -2.06080200 & -2.17422500 & 0.36328100\end{array}$

$\begin{array}{lrrr}\mathrm{N} & -2.65634500 & 1.47011900 & -0.26312600 \\ \mathrm{C} & -3.36783000 & -2.58520700 & -0.19908300 \\ \mathrm{C} & -1.26892800 & -3.25380400 & 1.01482600 \\ \mathrm{C} & -4.10785400 & 1.34249900 & -0.50545200 \\ \mathrm{C} & -2.01512300 & 2.79969900 & -0.40022900 \\ \mathrm{H} & -3.31131500 & -3.67469300 & -0.23874000 \\ \mathrm{C} & -3.53405000 & -2.10709900 & -1.63660900 \\ \mathrm{C} & -4.50957200 & -2.22643800 & 0.74865700 \\ \mathrm{C} & -0.28469400 & -2.72364300 & 2.04655400 \\ \mathrm{H} & -2.02338000 & -3.83983600 & 1.55111900 \\ \mathrm{C} & -0.59689900 & -4.14387000 & -0.02512000 \\ \mathrm{H} & -4.33242900 & 0.28992500 & -0.34679900 \\ \mathrm{C} & -4.91072100 & 2.15266000 & 0.50785900 \\ \mathrm{C} & -4.45936900 & 1.68716200 & -1.95021900 \\ \mathrm{H} & -2.79677300 & 3.45025700 & -0.79416100 \\ \mathrm{C} & -1.58748300 & 3.35822100 & 0.95478100 \\ \mathrm{C} & -0.87971000 & 2.76122000 & -1.41845100 \\ \mathrm{H} & -3.55229200 & -1.02019900 & -1.72275100 \\ \mathrm{H} & -2.71233200 & -2.47646300 & -2.25343400 \\ \mathrm{H} & -4.47149300 & -2.48778600 & -2.04664500 \\ \mathrm{H} & -4.37171000 & -2.71664200 & 1.71509100 \\ \mathrm{H} & -4.57462000 & -1.15175600 & 0.93128800 \\ \mathrm{H} & -5.46323000 & -2.55888400 & 0.33366700 \\ \mathrm{H} & 0.56781500 & -2.23108200 & 1.57800400 \\ \mathrm{H} & -0.76337700 & -2.03458200 & 2.74666200 \\ \mathrm{H} & 0.10129200 & -3.56841400 & 2.61960500 \\ \mathrm{H} & -1.31431200 & -4.53198700 & -0.75203500\end{array}$




$\begin{array}{rrrrrrrr}\mathrm{H} & 0.17284000 & -3.58465900 & -0.55879300 & \mathrm{H} & -5.51435800 & 1.47179100 & -2.13104500 \\ \mathrm{H} & -0.13107900 & -4.99904600 & 0.46949100 & \mathrm{H} & -1.24976200 & 4.39022000 & 0.83437800 \\ \mathrm{H} & -4.73549200 & 3.22610600 & 0.39784100 & \mathrm{H} & -2.41814600 & 3.35346300 & 1.66267800 \\ \mathrm{H} & -5.97753200 & 1.97860400 & 0.35375900 & \mathrm{H} & -0.76001100 & 2.79117900 & 1.38830100 \\ \mathrm{H} & -4.66050300 & 1.86288000 & 1.53095500 & \mathrm{H} & -0.06096900 & 2.11639600 & -1.08851600 \\ \mathrm{H} & -4.30184600 & 2.74637900 & -2.16764000 & \mathrm{H} & -1.23325400 & 2.39093200 & -2.38239400 \\ \mathrm{H} & -3.86319000 & 1.10006100 & -2.65206200 & \mathrm{H} & -0.47895900 & 3.76688400 & -1.56352200\end{array}$

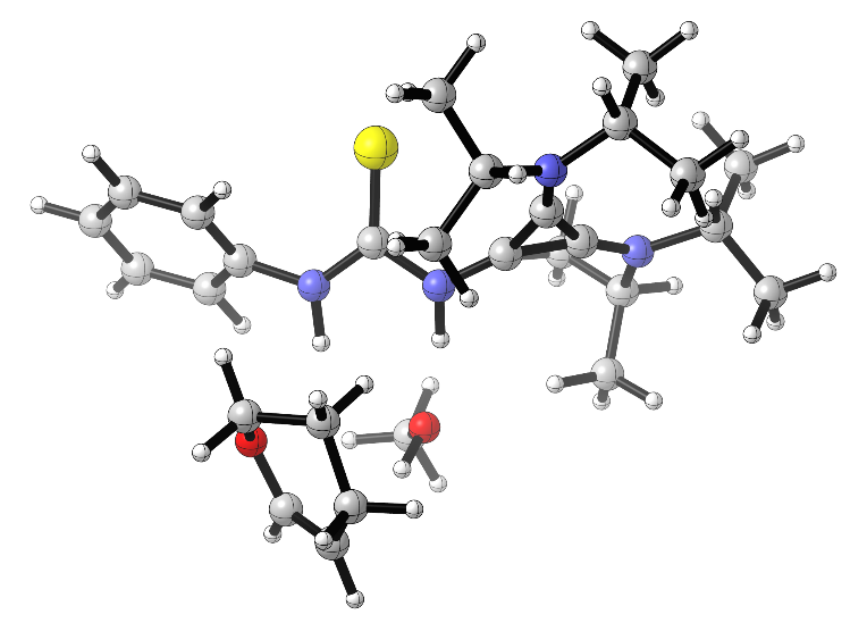

Figure S9. Optimized Structure of $Z, Z$-1-INT 2.

- Thermochemistry -

(0 imaginary frequencies)

Zero-point correction $=$

Thermal correction to Energy=

Thermal correction to Enthalpy=

Thermal correction to Gibbs Free Energy=

Sum of electronic and zero-point Energies=

Sum of electronic and thermal Energies=

Sum of electronic and thermal Enthalpies=

Sum of electronic and thermal Free Energies=
0.728118 (Hartree/Particle)

0.769320

0.770265
0.652003

$-1861.890966$

$-1861.849764$

$-1861.848820$

$-1861.967081$

$\begin{array}{lrrr}\mathrm{H} & -3.83026600 & 0.78646100 & 1.53705600 \\ \mathrm{O} & -4.05363600 & -1.20293800 & 1.27646900 \\ \mathrm{H} & -4.39731200 & -3.19960400 & 1.28514700 \\ \mathrm{H} & -3.12549700 & -3.67541500 & 3.32070000 \\ \mathrm{O} & -1.30467400 & -2.73023100 & 0.26825200 \\ \mathrm{H} & -1.83452300 & -2.90146900 & 1.06094200 \\ \mathrm{C} & -1.79552200 & -3.50722400 & -0.81724600 \\ \mathrm{H} & -2.85885200 & -3.31661900 & -0.99418800 \\ \mathrm{H} & -1.23445200 & -3.21059100 & -1.70406800 \\ \mathrm{H} & -1.64071800 & -4.57486000 & -0.64093800 \\ \mathrm{C} & -3.40997300 & 0.81939300 & -1.29677400 \\ \mathrm{C} & -3.70297600 & 2.16121100 & -1.08193600 \\ \mathrm{C} & -4.27023700 & 0.02086500 & -2.04039800 \\ \mathrm{C} & -4.85519400 & 2.70398800 & -1.63324000 \\ \mathrm{H} & -3.02934900 & 2.77507600 & -0.49876700 \\ \mathrm{C} & -5.42587300 & 0.56821800 & -2.58011500\end{array}$




$\begin{array}{lrrrrrrr}\mathrm{H} & -4.03043100 & -1.02478500 & -2.19731600 & \mathrm{C} & 1.77976800 & -1.97696600 & -2.64059600 \\ \mathrm{C} & -5.71724200 & 1.91178600 & -2.38185300 & \mathrm{H} & 4.55651000 & 1.46441800 & -0.98069700 \\ \mathrm{H} & -6.61532500 & 2.34119000 & -2.80933400 & \mathrm{H} & 3.92339000 & 3.10588700 & -1.07496100 \\ \mathrm{H} & -5.07970700 & 3.75184700 & -1.47388300 & \mathrm{H} & 5.56949000 & 2.81929100 & -0.48510200 \\ \mathrm{H} & -6.09386900 & -0.05464200 & -3.16280000 & \mathrm{H} & 4.51223100 & 1.66333100 & 2.93920200 \\ \mathrm{C} & 1.20075700 & -0.00343900 & -0.01787100 & \mathrm{H} & 4.84508300 & 0.46934800 & 1.68791400 \\ \mathrm{C} & 2.20971700 & 0.82377100 & 0.40737400 & \mathrm{H} & 5.91555600 & 1.86403500 & 1.88275700 \\ \mathrm{C} & 2.48590600 & -0.40350900 & -0.25586000 & \mathrm{H} & -0.24708300 & 1.85331100 & 1.45934900 \\ \mathrm{~N} & 2.61119700 & 1.92790400 & 0.98831500 & \mathrm{H} & 0.71725900 & 1.11014000 & 2.75879300 \\ \mathrm{~N} & 3.28617800 & -1.30346400 & -0.78176700 & \mathrm{H} & -0.05288700 & 2.68149900 & 2.99924800 \\ \mathrm{C} & 4.02678700 & 2.35917100 & 0.94827800 & \mathrm{H} & 2.22273100 & 4.59619500 & 0.66907800 \\ \mathrm{C} & 1.69071000 & 2.79323500 & 1.77346500 & \mathrm{H} & 0.73852900 & 3.79705000 & 0.11677900 \\ \mathrm{C} & 4.75524100 & -1.22968900 & -0.65244800 & \mathrm{H} & 0.74874000 & 4.72613800 & 1.63017000 \\ \mathrm{C} & 2.68082500 & -2.45004000 & -1.50423100 & \mathrm{H} & 5.15056800 & -3.35679300 & -0.37065800 \\ \mathrm{H} & 4.00690200 & 3.38281700 & 1.32740200 & \mathrm{H} & 6.37658600 & -2.28169800 & 0.29703500 \\ \mathrm{C} & 4.54785800 & 2.43438300 & -0.48240100 & \mathrm{H} & 4.82526100 & -2.46934500 & 1.12956300 \\ \mathrm{C} & 4.87439200 & 1.53674800 & 1.91630100 & \mathrm{H} & 5.31159400 & -1.97807800 & -2.62884000 \\ \mathrm{C} & 0.45711600 & 2.05142600 & 2.26807700 & \mathrm{H} & 5.00346900 & -0.23479000 & -2.56870400 \\ \mathrm{H} & 2.28086600 & 3.07190800 & 2.65357600 & \mathrm{H} & 6.49205000 & -0.90601500 & -1.88327000 \\ \mathrm{C} & 1.33165900 & 4.05491400 & 0.99550200 & \mathrm{H} & 1.62699800 & -4.28272600 & -1.08706600 \\ \mathrm{H} & 4.94859800 & -0.32149300 & -0.08589000 & \mathrm{H} & 2.66801200 & -3.74705900 & 0.23713300 \\ \mathrm{C} & 5.30211100 & -2.40704100 & 0.14870200 & \mathrm{H} & 1.11497300 & -2.94676900 & -0.06158600 \\ \mathrm{C} & 5.42234600 & -1.07937300 & -2.01729700 & \mathrm{H} & 0.89173700 & -1.45920900 & -2.26964100 \\ \mathrm{H} & 3.52691700 & -2.97661800 & -1.94762500 & \mathrm{H} & 2.31482200 & -1.29810400 & -3.30712700 \\ \mathrm{C} & 1.98278200 & -3.40580600 & -0.54104200 & \mathrm{H} & 1.44647200 & -2.83768700 & -3.22446000\end{array}$

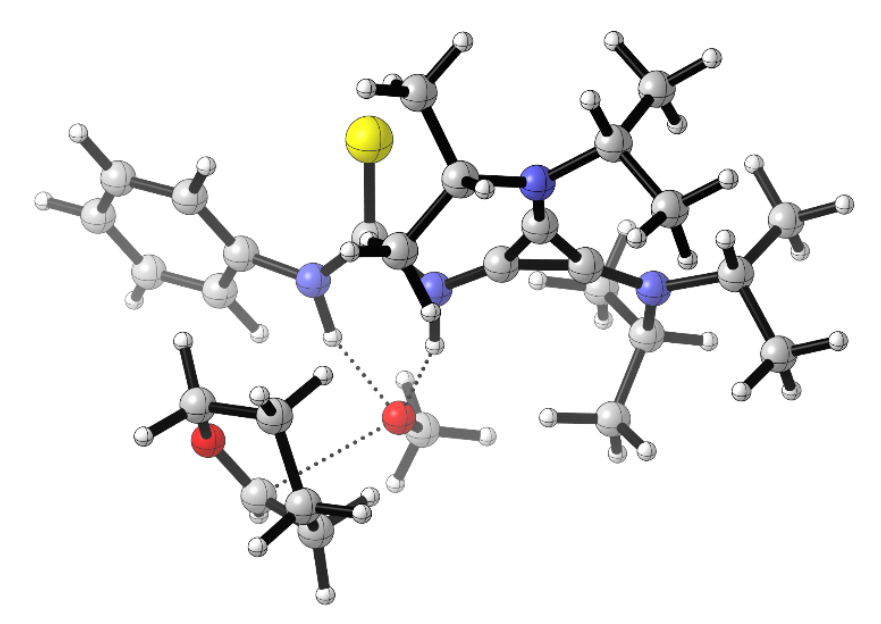

Figure S10. Optimized Structure of Z,Z-1-TS.

- Thermochemistry -

$(1$ imaginary frequency $=-303.24 \mathrm{~Hz})$

Zero-point correction $=$

Thermal correction to Energy=

Thermal correction to Enthalpy=

Thermal correction to Gibbs Free Energy=
0.723846 (Hartree/Particle)

0.763874

0.764818

0.650166 
Sum of electronic and zero-point Energies= Sum of electronic and thermal Energies $=$ Sum of electronic and thermal Enthalpies= Sum of electronic and thermal Free Energies=
$-1861.840949$

$-1861.800921$

$-1861.799977$

$-1861.914630$

$\begin{array}{lrrr}\mathrm{C} & -1.03874800 & 0.63428600 & -0.82301800 \\ \mathrm{~S} & -0.58966100 & 2.05376400 & -1.56504700 \\ \mathrm{~N} & -2.27463700 & 0.09674500 & -0.73579700 \\ \mathrm{H} & -2.26619900 & -0.87776400 & -0.37925000 \\ \mathrm{~N} & -0.11993600 & -0.21790500 & -0.21854500 \\ \mathrm{H} & -0.48773500 & -1.20829100 & -0.02925600 \\ \mathrm{C} & -3.68981100 & -2.04946500 & 1.90988600 \\ \mathrm{C} & -2.58791800 & -2.27772000 & 2.80384100 \\ \mathrm{C} & -2.15130600 & -1.06712200 & 3.61653400 \\ \mathrm{C} & -2.29548500 & 0.18563700 & 2.75134900 \\ \mathrm{C} & -3.70964000 & 0.31804400 & 2.23324000 \\ \mathrm{H} & -2.75644100 & -0.97593100 & 4.52355900 \\ \mathrm{H} & -1.11569100 & -1.19444200 & 3.93343200 \\ \mathrm{H} & -2.06549300 & 1.08976600 & 3.31936100 \\ \mathrm{H} & -1.60108500 & 0.14390600 & 1.90920000 \\ \mathrm{H} & -4.43761600 & 0.52237700 & 3.01960200 \\ \mathrm{H} & -3.81654100 & 1.04807400 & 1.43381400 \\ \mathrm{O} & -4.19004600 & -0.93184800 & 1.60687300 \\ \mathrm{H} & -4.14334000 & -2.87466000 & 1.36394000 \\ \mathrm{H} & -2.71018700 & -3.21673100 & 3.34588800 \\ \mathrm{O} & -1.54265800 & -2.32556400 & 0.26082900 \\ \mathrm{H} & -1.85486800 & -2.50234000 & 1.94030300 \\ \mathrm{C} & -1.69613700 & -3.38421200 & -0.63505900 \\ \mathrm{H} & -2.43504700 & -4.12303400 & -0.27868200 \\ \mathrm{H} & -2.04278000 & -3.04086300 & -1.62462200 \\ \mathrm{H} & -0.75952400 & -3.93523500 & -0.80251000 \\ \mathrm{C} & -3.50010400 & 0.55129300 & -1.26803500 \\ \mathrm{C} & -3.87146500 & 1.89402500 & -1.30063300 \\ \mathrm{C} & -4.40177000 & -0.42642400 & -1.69160000 \\ \mathrm{C} & -5.13253800 & 2.24373300 & -1.76836100 \\ \mathrm{H} & -3.17526000 & 2.65639900 & -0.98235200 \\ \mathrm{C} & -5.65950500 & -0.06617200 & -2.14940500 \\ \mathrm{H} & -4.10342300 & -1.47004100 & -1.67110700 \\ \mathrm{C} & -6.02990200 & 1.27297800 & -2.19247900 \\ \mathrm{H} & -7.00892400 & 1.55666400 & -2.55926400 \\ \mathrm{H} & -5.41019000 & 3.29076700 & -1.80292800 \\ \mathrm{H} & -6.34847400 & -0.83285700 & -2.48393100 \\ \mathrm{C} & 1.19811500 & 0.05922900 & -0.14336100 \\ \mathrm{~N} & 2.24808000 & 0.89469500 & 0.16124300 \\ & 2.47175600 & -0.42638100 & -0.28810300 \\ & 2.71518700 & 2.05128400 & 0.58523600\end{array}$

$\begin{array}{lrrr}\mathrm{N} & 3.23389900 & -1.44353300 & -0.63469600 \\ \mathrm{C} & 4.14624700 & 2.39648400 & 0.45121700 \\ \mathrm{C} & 1.89339100 & 3.00992400 & 1.36684400 \\ \mathrm{C} & 4.70416200 & -1.40265300 & -0.52027100 \\ \mathrm{C} & 2.58036200 & -2.69998800 & -1.07462600 \\ \mathrm{H} & 4.18845100 & 3.46760200 & 0.66050300 \\ \mathrm{C} & 4.62946800 & 2.22279700 & -0.98482900 \\ \mathrm{C} & 4.98249800 & 1.69578000 & 1.52182000 \\ \mathrm{C} & 0.67509900 & 2.35658000 & 2.00484600 \\ \mathrm{H} & 2.55911500 & 3.32174900 & 2.17998300 \\ \mathrm{C} & 1.52726700 & 4.23757600 & 0.53927300 \\ \mathrm{H} & 4.93851800 & -0.40179800 & -0.16629400 \\ \mathrm{C} & 5.21649200 & -2.39624600 & 0.51838600 \\ \mathrm{C} & 5.36690500 & -1.57740300 & -1.88403400 \\ \mathrm{H} & 3.40117300 & -3.34530600 & -1.39126000 \\ \mathrm{C} & 1.86651400 & -3.38554800 & 0.08862400 \\ \mathrm{C} & 1.67793100 & -2.47026800 & -2.28237400 \\ \mathrm{H} & 4.54949000 & 1.19489400 & -1.34070400 \\ \mathrm{H} & 4.03745000 & 2.85003200 & -1.65418500 \\ \mathrm{H} & 5.67633300 & 2.52221800 & -1.06466100 \\ \mathrm{H} & 4.67399800 & 2.02061300 & 2.51842500 \\ \mathrm{H} & 4.87403000 & 0.61018100 & 1.48431300 \\ \mathrm{H} & 6.04039200 & 1.93800000 & 1.40090100 \\ \mathrm{H} & -0.07859600 & 2.11046400 & 1.25620900 \\ \mathrm{H} & 0.94687800 & 1.45412500 & 2.55938200 \\ \mathrm{H} & 0.23072500 & 3.06548800 & 2.70652500 \\ \mathrm{H} & 2.41121400 & 4.69908900 & 0.09293100 \\ \mathrm{H} & 0.83743500 & 3.96109900 & -0.25798200 \\ \mathrm{H} & 1.04958800 & 4.98410000 & 1.17828200 \\ \mathrm{H} & 5.02975900 & -3.43087400 & 0.21931700 \\ \mathrm{H} & 6.29543500 & -2.27794200 & 0.63837800 \\ \mathrm{H} & 4.74353900 & -2.22703600 & 1.48873400 \\ \mathrm{H} & 5.20525000 & -2.57717500 & -2.29408200 \\ \mathrm{H} & 4.98581700 & -0.84705700 & -2.60127900 \\ \mathrm{H} & 6.44514500 & -1.43381700 & -1.78753000 \\ \mathrm{H} & 1.52966300 & -4.37745300 & -0.22072000 \\ \mathrm{H} & 2.53935600 & -3.50707200 & 0.93981200 \\ \mathrm{H} & 0.98242200 & -2.82926500 & 0.41309900 \\ \mathrm{H} & 0.81008700 & -1.85561200 & -2.03311500 \\ & 2.22592100 & -1.98352800 & -3.09161900 \\ 1.30509300 & -3.42932900 & -2.64830400\end{array}$




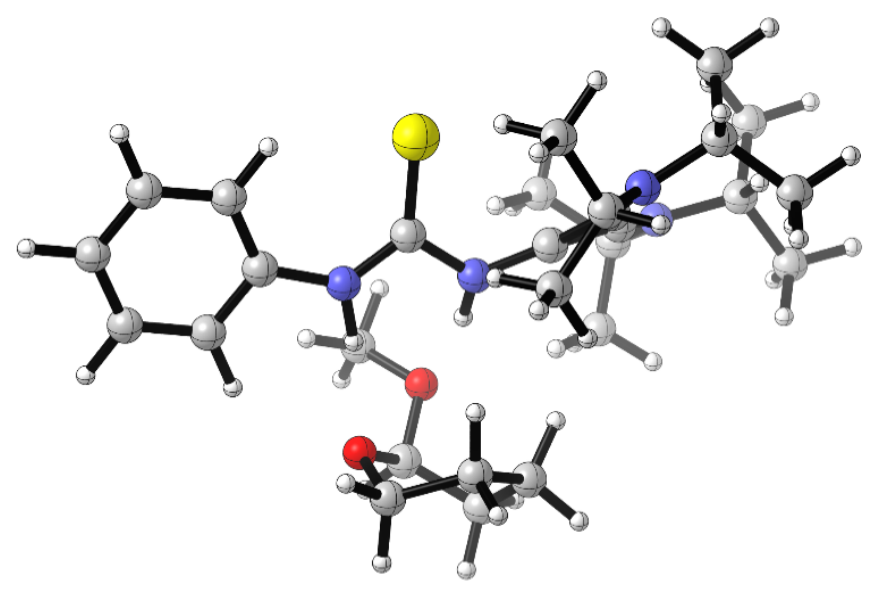

Figure S11. Optimized Structure of Z,Z-1-INT 3.

- Thermochemistry -

(0 imaginary frequencies)

Zero-point correction $=$

Thermal correction to Energy=

Thermal correction to Enthalpy=

Thermal correction to Gibbs Free Energy=

Sum of electronic and zero-point Energies=

Sum of electronic and thermal Energies $=$

Sum of electronic and thermal Enthalpies=

Sum of electronic and thermal Free Energies=
0.731375 (Hartree/Particle)

0.771006

0.771951

0.658826

$-1861.908964$

$-1861.869333$

$-1861.868389$

$-1861.981514$

$\begin{array}{lrrr}\mathrm{C} & -1.26281000 & 0.87192900 & -0.58405400 \\ \mathrm{~S} & -0.72057700 & 2.20617800 & -1.41042800 \\ \mathrm{~N} & -2.52067400 & 0.51367700 & -0.27902800 \\ \mathrm{H} & -2.61206800 & -0.33679400 & 0.27717600 \\ \mathrm{~N} & -0.34986700 & -0.09165900 & -0.11708400 \\ \mathrm{H} & -0.64901000 & -1.07216000 & -0.14663700 \\ \mathrm{C} & -2.22449500 & -3.06902800 & 0.82971900 \\ \mathrm{C} & -1.23625600 & -3.54302300 & 1.88194800 \\ \mathrm{C} & -0.47786100 & -2.38475900 & 2.53752700 \\ \mathrm{C} & -1.43977900 & -1.25971900 & 2.98790900 \\ \mathrm{C} & -2.88203800 & -1.59928200 & 2.64349100 \\ \mathrm{H} & 0.09127500 & -2.77269900 & 3.38393000 \\ \mathrm{H} & 0.26057100 & -1.98550000 & 1.83634500 \\ \mathrm{H} & -1.36584600 & -1.07936100 & 4.06296200 \\ \mathrm{H} & -1.17932500 & -0.32364300 & 2.48818200 \\ \mathrm{H} & -3.27417900 & -2.40599600 & 3.27138600 \\ \mathrm{H} & -3.53780600 & -0.73712700 & 2.76718100 \\ \mathrm{O} & -3.00107600 & -1.96268200 & 1.26372700 \\ \mathrm{H} & -2.91540900 & -3.87078700 & 0.54314000 \\ \mathrm{H} & -1.78977800 & -4.10247800 & 2.64075600 \\ \mathrm{O} & -1.51461200 & -2.66807500 & -0.31870100 \\ \mathrm{H} & -0.55401300 & -4.25024900 & 1.40710900 \\ \mathrm{C} & -2.28153900 & -2.65779800 & -1.52154200 \\ \mathrm{H} & -2.63241800 & -3.66696500 & -1.75489600 \\ \mathrm{H} & -3.13456700 & -1.97734400 & -1.44905000\end{array}$

$\begin{array}{lrrr}\mathrm{H} & -1.61520600 & -2.31591200 & -2.31270100 \\ \mathrm{C} & -3.78040400 & 1.03596200 & -0.67117700 \\ \mathrm{C} & -3.97815500 & 2.26491600 & -1.29489100 \\ \mathrm{C} & -4.88027700 & 0.22597800 & -0.36576100 \\ \mathrm{C} & -5.27197400 & 2.66146200 & -1.61639000 \\ \mathrm{H} & -3.14098700 & 2.90365500 & -1.52539700 \\ \mathrm{C} & -6.16175700 & 0.63872400 & -0.68899000 \\ \mathrm{H} & -4.72354500 & -0.72643200 & 0.13028500 \\ \mathrm{C} & -6.36465300 & 1.86076300 & -1.32002800 \\ \mathrm{H} & -7.36648200 & 2.18521200 & -1.57389900 \\ \mathrm{H} & -5.41804600 & 3.61854200 & -2.10310600 \\ \mathrm{H} & -7.00405300 & 0.00183600 & -0.44581800 \\ \mathrm{C} & 0.97959800 & 0.16620100 & -0.05939500 \\ \mathrm{C} & 2.00978400 & 0.98066500 & 0.34489000 \\ \mathrm{C} & 2.25610600 & -0.21742200 & -0.37091700 \\ \mathrm{~N} & 2.43710000 & 2.06938800 & 0.94061200 \\ \mathrm{~N} & 3.04107600 & -1.09056500 & -0.96532100 \\ \mathrm{C} & 3.83556100 & 2.53490800 & 0.80754100 \\ \mathrm{C} & 1.57676400 & 2.86943400 & 1.85282900 \\ \mathrm{C} & 4.51225500 & -1.02535000 & -0.85716000 \\ \mathrm{C} & 2.42215900 & -2.21163600 & -1.71295900 \\ \mathrm{H} & 3.81392900 & 3.55822800 & 1.18783900 \\ \mathrm{C} & 4.26018100 & 2.62514300 & -0.65357500 \\ \mathrm{C} & 4.76815700 & 1.73587600 & 1.71543400 \\ \mathrm{C} & 0.42537100 & 2.06200200 & 2.43324000\end{array}$




$\begin{array}{lrrrrrrr}\mathrm{H} & 2.24590900 & 3.12549600 & 2.68170300 & \mathrm{H} & -0.03427300 & 2.63810700 & 3.23810400 \\ \mathrm{C} & 1.10539100 & 4.15734900 & 1.18654200 & \mathrm{H} & 1.94100200 & 4.72848700 & 0.77524800 \\ \mathrm{H} & 4.71753300 & -0.13114100 & -0.27284500 & \mathrm{H} & 0.40677900 & 3.93216800 & 0.37975400 \\ \mathrm{C} & 5.06812200 & -2.22230100 & -0.09168300 & \mathrm{H} & 0.60069500 & 4.78840100 & 1.92155100 \\ \mathrm{C} & 5.16190200 & -0.84631700 & -2.22696200 & \mathrm{H} & 4.90666200 & -3.15930200 & -0.63093900 \\ \mathrm{H} & 3.25744300 & -2.71355900 & -2.20311100 & \mathrm{H} & 6.14493700 & -2.10282700 & 0.04420300 \\ \mathrm{C} & 1.75905200 & -3.21186200 & -0.77041200 & \mathrm{H} & 4.60537300 & -2.30640400 & 0.89440700 \\ \mathrm{C} & 1.48331000 & -1.69791600 & -2.79962000 & \mathrm{H} & 5.04815700 & -1.73317700 & -2.85498500 \\ \mathrm{H} & 4.27320500 & 1.65597300 & -1.15293700 & \mathrm{H} & 4.73400600 & 0.00730100 & -2.75699000 \\ \mathrm{H} & 3.57636100 & 3.27426700 & -1.20413900 & \mathrm{H} & 6.23245400 & -0.67151700 & -2.10185700 \\ \mathrm{H} & 5.26519800 & 3.04612200 & -0.72105400 & \mathrm{H} & 1.42452500 & -4.08495800 & -1.33535200 \\ \mathrm{H} & 4.47503200 & 1.85273600 & 2.76138200 & \mathrm{H} & 2.45676100 & -3.54846400 & -0.00130200 \\ \mathrm{H} & 4.75307900 & 0.66809200 & 1.48770000 & \mathrm{H} & 0.87575800 & -2.78893200 & -0.28903900 \\ \mathrm{H} & 5.79514000 & 2.09205300 & 1.61153200 & \mathrm{H} & 0.61880600 & -1.17786000 & -2.37892700 \\ \mathrm{H} & -0.34842900 & 1.86835900 & 1.68919000 & \mathrm{H} & 2.00090100 & -1.00743800 & -3.46798600 \\ \mathrm{H} & 0.77087300 & 1.11367900 & 2.85302000 & \mathrm{H} & 1.11407600 & -2.53777100 & -3.39206300\end{array}$




\section{Pathway 2 (Brønsted Acid-Catalyzed Mechanism)}

\section{a. Anilino Nitrogen Protonation}

Scheme S2. Representative Scheme for Pathway 2: Brønsted Acid Catalysis Anilino N(4) Protonation Featuring Conformer E,Z-1. See Table S1 for energies of E,E-1, Z,Z-1 and Z,E-1 pathways.

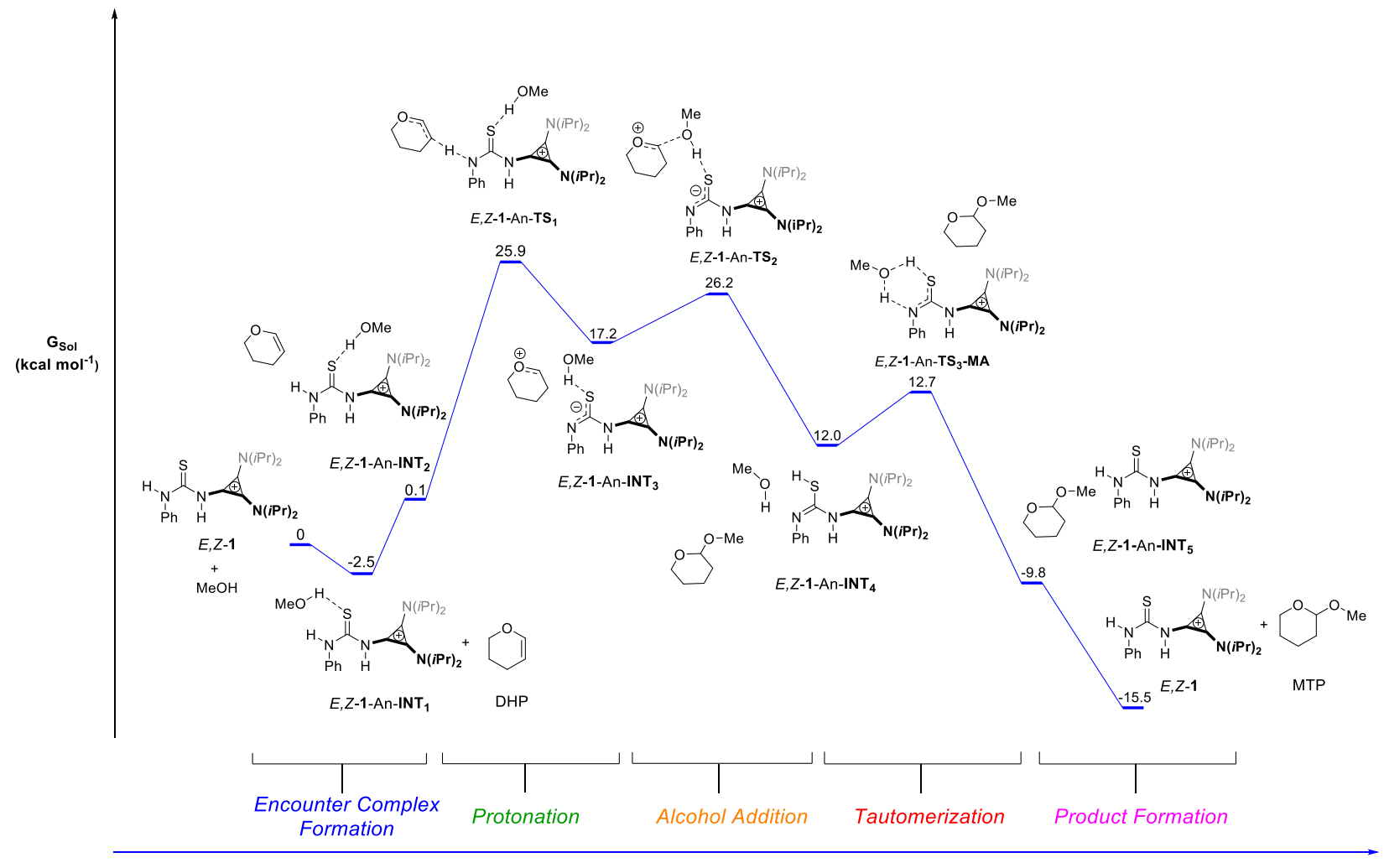

Pathway 2 - Brønsted Acid Catalysis

Table S1. Relative Energies of Conformations for Pathway 2 - Anilino N(4) Protonation.

\begin{tabular}{|c|c|c|c|c|c|c|c|c|c|c|c|}
\hline & \multicolumn{10}{|c|}{$\Delta \mathrm{G}_{\mathrm{Sol}}\left(\mathrm{kcal} / \mathrm{mol}^{\prime}\right)$} \\
\hline Conformer & $\begin{array}{c}\text { Pre- } \\
\text { Eq. }\end{array}$ & $\mathrm{INT}_{1}$ & $\mathrm{INT}_{2}$ & $\mathrm{TS}_{1}$ & $\mathrm{INT}_{3}$ & $\mathrm{TS}_{2}$ & $\mathrm{INT}_{4}$ & $\begin{array}{l}\mathrm{TS}_{3} \\
\mathrm{PA}\end{array}$ & $\begin{array}{c}\mathrm{TS}_{3} \\
\mathrm{MA}\end{array}$ & $\mathrm{INT}_{5}$ & $\begin{array}{c}\text { Prod. } \\
\text { Diff. }\end{array}$ \\
\hline$E, E-1$ & 0.0 & -1.1 & 4.8 & 26.6 & 10.2 & 25.2 & 9.5 & 21.4 & 14.8 & -7.4 & -12.7 \\
\hline$E, Z-1$ & 0.0 & -2.5 & 0.1 & 25.9 & 17.2 & 26.2 & 12.0 & 21.5 & 12.7 & -9.8 & -15.5 \\
\hline$Z, Z-1$ & 0.0 & -1.3 & -0.8 & 26.9 & 24.8 & 36.9 & 5.9 & 29.5 & 30.0 & -7.5 & -13.2 \\
\hline$Z, E-1$ & 0.0 & 2.9 & 4.1 & 28.3 & 26.6 & 28.4 & 6.6 & 27.1 & 30.0 & -10.4 & -8.2 \\
\hline
\end{tabular}

- "An" added to names in SI to differentiate mode of catalysis. 


\section{i. E,E-Conformation}

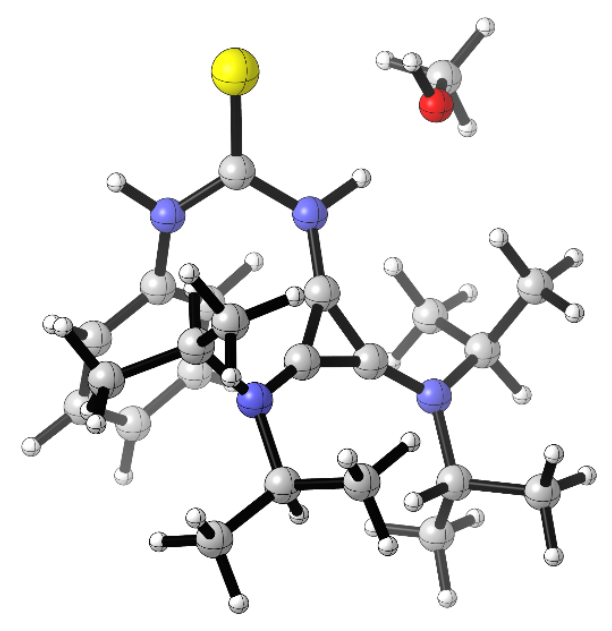

Figure S12. Optimized Structure of E,E-1-An-INT 1 .

- Thermochemistry -

(0 imaginary frequencies)

Zero-point correction $=$

Thermal correction to Energy=

Thermal correction to Enthalpy=

Thermal correction to Gibbs Free Energy=
Sum of electronic and zero-point Energies=

Sum of electronic and thermal Energies=

Sum of electronic and thermal Enthalpies=

Sum of electronic and thermal Free Energies=
0.602894 (Hartree/Particle)

0.636314

0.637258

0.537826

$-1591.464198$

$-1591.430778$

$-1591.429834$

$-1591.529266$

$\begin{array}{lrrr}\mathrm{C} & -1.70543000 & 2.14334800 & -0.50602100 \\ \mathrm{~S} & -2.81206300 & 2.97260100 & -1.41891200 \\ \mathrm{~N} & -0.81954300 & 2.77203700 & 0.30901300 \\ \mathrm{H} & -0.85067700 & 3.77728200 & 0.21223000 \\ \mathrm{~N} & -1.64481500 & 0.75966400 & -0.53741500 \\ \mathrm{C} & -0.21201300 & 2.28230300 & 1.50170700 \\ \mathrm{C} & 1.00870000 & 2.83030900 & 1.88298900 \\ \mathrm{C} & -0.82810900 & 1.33091200 & 2.31128700 \\ \mathrm{C} & 1.63171500 & 2.40014300 & 3.04611900 \\ \mathrm{H} & 1.46052900 & 3.60261500 & 1.27166900 \\ \mathrm{C} & -0.18955800 & 0.89268200 & 3.46331000 \\ \mathrm{H} & -1.80727800 & 0.94861200 & 2.05486100 \\ \mathrm{C} & 1.04392100 & 1.41622700 & 3.83160600 \\ \mathrm{H} & 1.53056900 & 1.07918500 & 4.73863800\end{array}$

$\begin{array}{lrrr}\mathrm{H} & -2.48346200 & 0.29438500 & -0.92728300 \\ \mathrm{C} & -5.04871500 & -0.27340500 & -0.73967900 \\ \mathrm{H} & -5.14040800 & 0.66378900 & -0.18185500 \\ \mathrm{H} & -4.84548300 & -1.08906500 & -0.04526000 \\ \mathrm{H} & -5.98617100 & -0.48226200 & -1.26002100 \\ \mathrm{O} & -3.95549100 & -0.22799900 & -1.65437000 \\ \mathrm{H} & -4.08651300 & 0.51402800 & -2.25335400 \\ \mathrm{C} & -0.51993000 & 0.01666200 & -0.40903100 \\ \mathrm{C} & 0.83622200 & -0.14865600 & -0.52650200 \\ \mathrm{C} & 0.01082400 & -1.21859300 & -0.13065000 \\ \mathrm{~N} & 2.02860800 & 0.32995500 & -0.82605000 \\ \mathrm{~N} & -0.17436100 & -2.47215300 & 0.21359600 \\ \mathrm{C} & 3.14149300 & -0.65867900 & -0.82936700 \\ \mathrm{C} & 2.04498700 & 1.64668800 & -1.52533700\end{array}$




$\begin{array}{lrrrrrrr}\mathrm{C} & 0.97030800 & -3.39417300 & 0.37478700 & \mathrm{H} & 2.04308400 & 2.46235500 & -3.51475100 \\ \mathrm{C} & -1.54519500 & -2.95067400 & 0.51595600 & \mathrm{H} & 0.35666600 & -4.27956200 & 2.27265300 \\ \mathrm{C} & 3.04910600 & -1.61346000 & -2.02201700 & \mathrm{H} & 2.08950000 & -4.33648400 & 1.96047900 \\ \mathrm{H} & 2.99121400 & -1.22701100 & 0.09297400 & \mathrm{H} & 1.33069200 & -2.80622600 & 2.43129800 \\ \mathrm{C} & 4.52547500 & -0.03505300 & -0.72477200 & \mathrm{H} & -0.01776300 & -5.26109400 & -0.18451500 \\ \mathrm{C} & 2.19528100 & 1.49251900 & -3.03637000 & \mathrm{H} & 0.67633300 & -4.36346500 & -1.54684500 \\ \mathrm{H} & 1.83716700 & -2.84272000 & 0.01451600 & \mathrm{H} & 1.72606300 & -5.24102100 & -0.42496100 \\ \mathrm{C} & 1.19482700 & -3.72056700 & 1.84883400 & \mathrm{H} & -3.15722900 & -2.55209900 & 1.88490400 \\ \mathrm{C} & 0.82093400 & -4.63496000 & -0.49895400 & \mathrm{H} & -1.59298500 & -1.92807400 & 2.43255800 \\ \mathrm{H} & -1.40435600 & -3.93874800 & 0.95605300 & \mathrm{H} & -2.49324100 & -1.09510300 & 1.14909200 \\ \mathrm{C} & -2.23156700 & -2.07372400 & 1.55870200 & \mathrm{H} & -2.55574700 & -2.14065700 & -1.24283300 \\ \mathrm{C} & -2.36614300 & -3.10334800 & -0.76063700 & \mathrm{H} & -1.86114100 & -3.75660300 & -1.47424900 \\ \mathrm{H} & 2.04951300 & -2.04480800 & -2.12383700 & \mathrm{H} & -3.33651100 & -3.54699300 & -0.52578500 \\ \mathrm{H} & 3.29249900 & -1.10684700 & -2.95695000 & \mathrm{H} & 2.57729400 & 2.84023700 & 3.33988000 \\ \mathrm{H} & 3.75844400 & -2.43455500 & -1.89238400 & \mathrm{H} & -0.67685600 & 0.15715500 & 4.09297400 \\ \mathrm{H} & 4.80591200 & 0.49706400 & -1.63513500 & \mathrm{H} & 1.05023400 & 2.04954500 & -1.32712400 \\ \mathrm{H} & 4.60485900 & 0.64209700 & 0.12528900 & \mathrm{C} & 3.04755100 & 2.64614300 & -0.94784200 \\ \mathrm{H} & 5.25008800 & -0.83950000 & -0.58445200 & \mathrm{H} & 4.04370600 & 2.53211800 & -1.37406200 \\ \mathrm{H} & 3.19525400 & 1.14629900 & -3.30734600 & \mathrm{H} & 2.70804700 & 3.65698600 & -1.18647900 \\ \mathrm{H} & 1.45929900 & 0.79436100 & -3.44149400 & \mathrm{H} & 3.11847400 & 2.55477900 & 0.13573900\end{array}$

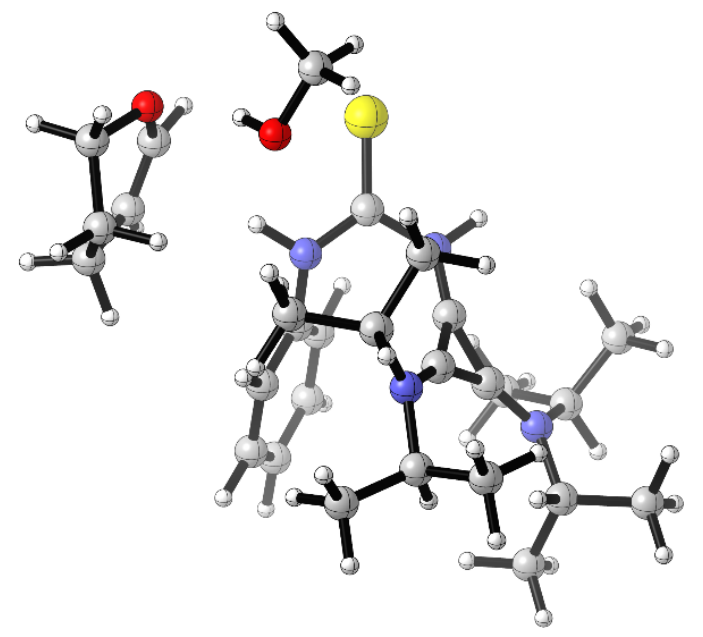

Figure S13. Optimized Structure of E,E-1-An-INT 2 .

- Thermochemistry -

(0 imaginary frequencies)

Zero-point correction $=$

Thermal correction to Energy=

0.727990 (Hartree/Particle)

Thermal correction to Enthalpy=

0.769375

Thermal correction to Gibbs Free Energy=

0.770319

Sum of electronic and zero-point Energies=

0.650709

Sum of electronic and thermal Energies=

$-1861.880770$

Sum of electronic and thermal Enthalpies=

$-1861.839385$

Sum of electronic and thermal Free Energies=

$-1861.838441$

$-1861.958051$ 


$\begin{array}{lrrr}\mathrm{C} & -0.99664100 & -1.11551400 & -1.52635600 \\ \mathrm{~S} & -2.03686700 & -1.40693500 & -2.77055800 \\ \mathrm{~N} & 0.37244700 & -0.98142500 & -1.77052700 \\ \mathrm{H} & 0.67887500 & -1.30420100 & -2.67625300 \\ \mathrm{~N} & -1.34853300 & -0.99699500 & -0.22212500 \\ \mathrm{C} & -0.57356700 & -1.50528500 & 0.87058200 \\ \mathrm{C} & 0.02437900 & -2.75883500 & 0.77878100 \\ \mathrm{C} & -0.47540900 & -0.77046100 & 2.04755100 \\ \mathrm{C} & -4.20580900 & -1.71162800 & 1.21641600 \\ \mathrm{C} & -4.30783200 & -0.66538300 & 2.29176300 \\ \mathrm{C} & -4.67239200 & 0.68437000 & 1.66965200 \\ \mathrm{C} & -5.73808100 & 0.49235400 & 0.60317600 \\ \mathrm{H} & -5.06007100 & -0.96319200 & 3.03063200 \\ \mathrm{H} & -3.36040000 & -0.59442600 & 2.83546600 \\ \mathrm{H} & -5.03471400 & 1.38651600 & 2.42382500 \\ \mathrm{H} & -3.79749600 & 1.13784900 & 1.19511700 \\ \mathrm{H} & -6.64502800 & 0.04974000 & 1.02892200 \\ \mathrm{H} & -5.99332500 & 1.42893900 & 0.11032900 \\ \mathrm{O} & -5.27725700 & -0.37303900 & -0.44520300 \\ \mathrm{H} & -4.56956100 & -2.23110900 & -0.82274200 \\ \mathrm{H} & -3.76215900 & -2.67438300 & 1.44249500 \\ \mathrm{C} & -3.76269700 & 1.99274100 & -2.86622600 \\ \mathrm{H} & -4.83027300 & 2.17480200 & -3.03473800 \\ \mathrm{H} & -3.40719100 & 1.26625400 & -3.60665900 \\ \mathrm{H} & -3.23227600 & 2.93367400 & -3.02465000 \\ \mathrm{O} & -3.49857700 & 1.56821300 & -1.54389400 \\ \mathrm{H} & -4.03131700 & 0.78094000 & -1.37893800 \\ \mathrm{H} & 0.30491600 & -0.71397000 & 4.04353900 \\ \mathrm{H} & 1.20698200 & -4.23806800 & 1.77789700 \\ \mathrm{C} & 1.21312700 & -0.21092600 & -1.03412100 \\ \mathrm{C} & 1.44204500 & 0.89453500 & -0.25219000 \\ \mathrm{C} & 2.48574900 & -0.00333000 & -0.56587200 \\ \mathrm{~N} & 1.04880900 & 1.99079800 & 0.35349700 \\ \mathrm{~N} & 3.74464000 & -0.36653800 & -0.51467200 \\ \mathrm{C} & 1.98626400 & 2.64188300 & 1.29860500 \\ \mathrm{C} & -0.14985800 & 2.74481500 & -0.11853000 \\ \mathrm{C} & 4.75201700 & 0.47764700 & 0.16351700 \\ \mathrm{C} & 4.15332600 & -1.65156800 & -1.13139100 \\ \mathrm{C} & 2.79256800 & 3.74121000 & 0.61001900 \\ \mathrm{C} & 2.66418800 & 1.84715100 & 1.61873200 \\ & 1.26855600 & 3.13752900 & 2.54850700\end{array}$

$\begin{array}{lrrr}\mathrm{C} & 0.74739500 & -3.25944900 & 1.85258800 \\ \mathrm{H} & -0.09175400 & -3.34166400 & -0.12819800 \\ \mathrm{C} & 0.23981200 & -1.28242600 & 3.12295400 \\ \mathrm{H} & -0.96056000 & 0.19371000 & 2.11848500 \\ \mathrm{C} & 0.86084400 & -2.52206500 & 3.02526200 \\ \mathrm{H} & 1.41274400 & -2.92177200 & 3.86755700 \\ \mathrm{H} & -2.35560200 & -1.02436300 & -0.06787100 \\ \mathrm{C} & -4.66373300 & -1.50926400 & -0.02070200 \\ \mathrm{C} & -1.41320700 & 2.38568600 & 0.64911600 \\ \mathrm{H} & 0.10003500 & 3.78893600 & 0.08459000 \\ \mathrm{C} & -0.34588100 & 2.60155100 & -1.62370200 \\ \mathrm{H} & 4.23440000 & 1.40608800 & 0.40340800 \\ \mathrm{C} & 5.20953500 & -0.17575600 & 1.46369900 \\ \mathrm{C} & 5.90673800 & 0.83142900 & -0.76857800 \\ \mathrm{H} & 5.19723000 & -1.78468400 & -0.84436500 \\ \mathrm{C} & 3.35719600 & -2.81897400 & -0.56213700 \\ \mathrm{C} & 4.08277500 & -1.56964200 & -2.65318500 \\ \mathrm{H} & 3.29237500 & 3.36487500 & -0.28666100 \\ \mathrm{H} & 2.15700800 & 4.57932700 & 0.31449000 \\ \mathrm{H} & 3.55342700 & 4.12937900 & 1.29082700 \\ \mathrm{H} & 0.56942200 & 3.94641600 & 2.32567200 \\ \mathrm{H} & 0.72421700 & 2.32474000 & 3.03301800 \\ \mathrm{H} & 2.00572100 & 3.52444900 & 3.25474100 \\ \mathrm{H} & -1.69696300 & 1.35820500 & 0.42636700 \\ \mathrm{H} & -1.28790100 & 2.51701000 & 1.72519900 \\ \mathrm{H} & -2.23703300 & 3.01410100 & 0.30833700 \\ \mathrm{H} & 0.58423200 & 2.78417500 & -2.16795100 \\ \mathrm{H} & -0.75045200 & 1.62319700 & -1.89072400 \\ \mathrm{H} & -1.08290100 & 3.33573200 & -1.95112500 \\ \mathrm{H} & 5.73283600 & -1.11717800 & 1.27595800 \\ \mathrm{H} & 5.89942800 & 0.48470900 & 1.99290100 \\ \mathrm{H} & 4.35797300 & -0.38238700 & 2.11675500 \\ \mathrm{H} & 5.54215800 & 1.30452900 & -1.68275600 \\ \mathrm{H} & 6.57501700 & 1.53246100 & -0.26486400 \\ \mathrm{H} & 6.50016000 & -0.04435900 & -1.04198900 \\ \mathrm{H} & 3.75991900 & -3.75894200 & -0.94462000 \\ \mathrm{H} & 3.41112000 & -2.83450000 & 0.52814900 \\ \mathrm{H} & 2.30424700 & -2.76449300 & -0.84906900 \\ & 3.05439200 & -1.42067000 & -2.99536800 \\ \mathrm{H} & 4.69067300 & -0.74730400 & -3.03391000 \\ & 4.44496300 & -2.49902700 & -3.09665200\end{array}$




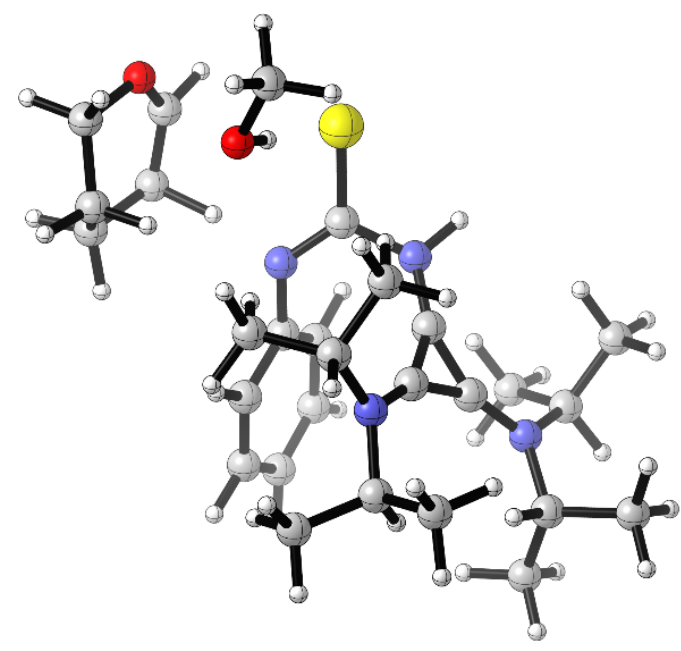

Figure S14. Optimized Structure of E,E-1-An-TS . $_{\text {. }}$

- Thermochemistry -

$(1$ imaginary frequency $=-506.37 \mathrm{~Hz})$

Zero-point correction=

Thermal correction to Energy=

Thermal correction to Enthalpy=

Thermal correction to Gibbs Free Energy=

Sum of electronic and zero-point Energies=

Sum of electronic and thermal Energies=

Sum of electronic and thermal Enthalpies=

Sum of electronic and thermal Free Energies=

$$
\begin{array}{rrr}
-1.17845900 & -0.87227500 & -1.24766900 \\
-2.36865700 & -0.66931800 & -2.44555200 \\
0.16893600 & -0.81907900 & -1.68713600 \\
0.29593500 & -0.93177100 & -2.68322100 \\
-1.42661500 & -1.07680100 & 0.01709700 \\
-0.47893800 & -1.60310100 & 0.92723100 \\
0.15970200 & -2.81928600 & 0.67154100 \\
-0.25444000 & -0.96733900 & 2.14959600 \\
1.03760900 & -3.36028500 & 1.60061100 \\
-0.04067200 & -3.33606300 & -0.26042300 \\
0.63174500 & -1.50858700 & 3.07164100 \\
-0.77620900 & -0.04610000 & 2.37090100 \\
1.28865300 & -2.70305900 & 2.79949900 \\
1.97002300 & -3.13048200 & 3.52539200 \\
-2.83048400 & -1.55225100 & 0.25078000 \\
-4.70948600 & -1.69662500 & -0.49096500 \\
-3.92840500 & -1.99327800 & 0.64043300 \\
-4.21947900 & -1.20030500 & 1.90366900 \\
-4.57815700 & 0.22654100 & 1.49298000 \\
-5.71017800 & 0.23494400 & 0.49000600 \\
-5.03555800 & -1.65871300 & 2.47073500 \\
-3.33756100 & -1.20192000 & 2.54765900 \\
-4.88579200 & 0.82623600 & 2.35257200 \\
-3.71464400 & 0.71884900 & 1.04130800 \\
-6.66074300 & -0.08328200 & 0.92238900
\end{array}
$$
2648 (Hartree/Particle)
0.763218
0.764162
0.648624
$-1861.852291$
$-1861.811721$
$-1861.810777$
$-1861.926315$

$\begin{array}{lrrr}\mathrm{H} & -5.81511700 & 1.20280300 & 0.00884100 \\ \mathrm{O} & -5.47876800 & -0.68371100 & -0.62990800 \\ \mathrm{H} & -4.67163100 & -2.31223600 & -1.38403300 \\ \mathrm{H} & -3.70878300 & -3.05566700 & 0.73967700 \\ \mathrm{C} & -4.07921300 & 2.81533500 & -2.22066500 \\ \mathrm{H} & -4.80093600 & 2.29647700 & -2.86204700 \\ \mathrm{H} & -3.21547600 & 3.10268100 & -2.83329000 \\ \mathrm{H} & -4.54719700 & 3.72301400 & -1.83711000 \\ \mathrm{O} & -3.69850400 & 2.02900500 & -1.10785300 \\ \mathrm{H} & -3.29940000 & 1.21678600 & -1.45365800 \\ \mathrm{H} & 0.79566700 & -1.00306600 & 4.01695500 \\ \mathrm{H} & 1.51983400 & -4.30827200 & 1.39012200 \\ \mathrm{C} & 1.11612100 & -0.14342900 & -0.99371700 \\ \mathrm{C} & 1.44710800 & 0.86893100 & -0.12668800 \\ \mathrm{C} & 2.43690300 & 0.01570100 & -0.65266900 \\ \mathrm{~N} & 1.12386300 & 1.89872700 & 0.62507300 \\ \mathrm{~N} & 3.69280200 & -0.34021700 & -0.78416600 \\ \mathrm{C} & 2.12226000 & 2.41702500 & 1.58538500 \\ \mathrm{C} & -0.09627600 & 2.70248600 & 0.32862500 \\ \mathrm{C} & 4.76864200 & 0.43893500 & -0.13687700 \\ \mathrm{C} & 4.02628100 & -1.53251500 & -1.59880500 \\ \mathrm{C} & 2.87184100 & 3.62173100 & 1.02078600 \\ \mathrm{H} & 2.82827900 & 1.59595400 & 1.73305700 \\ \mathrm{C} & 1.48579400 & 2.70147100 & 2.94128200 \\ \mathrm{C} & -1.32966900 & 2.20974300 & 1.07028900\end{array}$




$\begin{array}{rrrrrrrr}\mathrm{H} & 0.15073000 & 3.70206200 & 0.69373400 & \mathrm{H} & -2.18091500 & 2.84495300 & 0.82216100 \\ \mathrm{C} & -0.34124300 & 2.81222900 & -1.17293200 & \mathrm{H} & 0.55938900 & 3.13857300 & -1.69913200 \\ \mathrm{H} & 4.27355300 & 1.31028200 & 0.29257200 & \mathrm{H} & -0.68967600 & 1.87047900 & -1.60381400 \\ \mathrm{C} & 5.40525100 & -0.36196100 & 0.99476200 & \mathrm{H} & -1.12956100 & 3.54525500 & -1.34950700 \\ \mathrm{C} & 5.78408700 & 0.94782100 & -1.15565100 & \mathrm{H} & 5.91875900 & -1.24997800 & 0.61686000 \\ \mathrm{H} & 5.09669100 & -1.68603700 & -1.45403100 & \mathrm{H} & 6.14482800 & 0.24919700 & 1.51620600 \\ \mathrm{C} & 3.29755400 & -2.77099700 & -1.09308300 & \mathrm{H} & 4.64955500 & -0.68348000 & 1.71548500 \\ \mathrm{C} & 3.77272100 & -1.26469600 & -3.07943900 & \mathrm{H} & 5.29495100 & 1.52796300 & -1.94112100 \\ \mathrm{H} & 3.31849600 & 3.39202700 & 0.04962000 & \mathrm{H} & 6.50883200 & 1.59295700 & -0.65505000 \\ \mathrm{H} & 2.21043000 & 4.48201200 & 0.89256200 & \mathrm{H} & 6.34093800 & 0.13174400 & -1.62243800 \\ \mathrm{H} & 3.66983600 & 3.92035700 & 1.70427500 & \mathrm{H} & 3.65391700 & -3.65260400 & -1.62977000 \\ \mathrm{H} & 0.75984800 & 3.51631000 & 2.88862900 & \mathrm{H} & 3.47068600 & -2.91811900 & -0.02547600 \\ \mathrm{H} & 0.98615300 & 1.81093500 & 3.32735800 & \mathrm{H} & 2.21972200 & -2.69126200 & -1.25476100 \\ \mathrm{H} & 2.26079900 & 2.99668300 & 3.65132200 & \mathrm{H} & 2.70860600 & -1.09769200 & -3.27105300 \\ \mathrm{H} & -1.58039300 & 1.19840500 & 0.74762900 & \mathrm{H} & 4.32490600 & -0.38924100 & -3.42524400 \\ \mathrm{H} & -1.17935200 & 2.21797300 & 2.15158200 & \mathrm{H} & 4.08255800 & -2.12534400 & -3.67540800\end{array}$

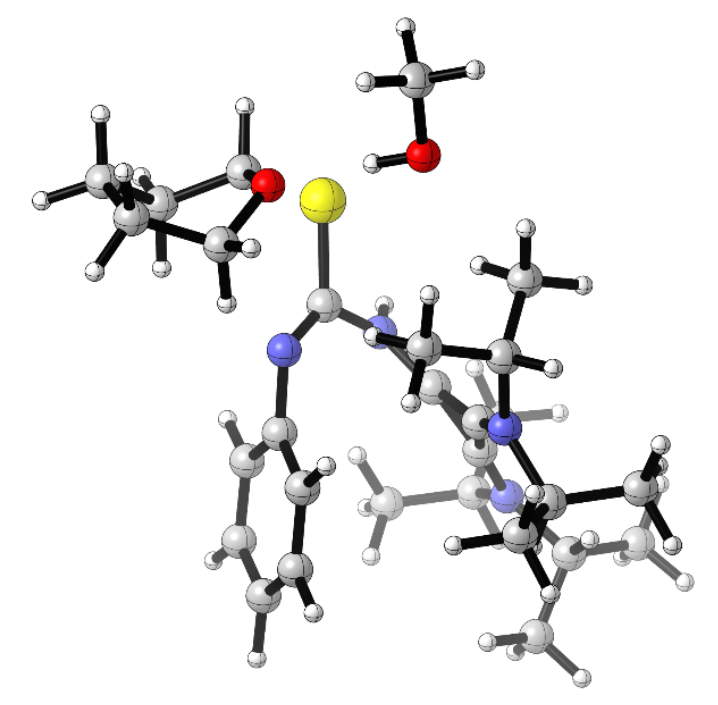

Figure S15. Optimized Structure of $E, E-1-A n-I N T_{3}$.

- Thermochemistry -

(0 imaginary frequencies)

Zero-point correction=

Thermal correction to Energy=

0.728170 (Hartree/Particle)

Thermal correction to Enthalpy=

0.768994

0.769939

Thermal correction to Gibbs Free Energy=

Sum of electronic and zero-point Energies=

0.653515

Sum of electronic and thermal Energies=

$-1861.878500$

Sum of electronic and thermal Enthalpies=

$-1861.837676$

Sum of electronic and thermal Free Energies=

$-1861.836732$

$-1861.953155$ 


$\begin{array}{lrrr}\mathrm{C} & -1.15184100 & -1.06528700 & -0.90962800 \\ \mathrm{~S} & -2.42445100 & -0.76569000 & -2.08346600 \\ \mathrm{~N} & 0.12775800 & -0.99968500 & -1.52077000 \\ \mathrm{H} & 0.27561300 & -1.41320900 & -2.42855900 \\ \mathrm{~N} & -1.33105800 & -1.24250500 & 0.32907500 \\ \mathrm{C} & -0.29108200 & -1.66473500 & 1.18817900 \\ \mathrm{C} & 0.31947200 & -2.90495500 & 0.99598200 \\ \mathrm{C} & 0.06448600 & -0.89193500 & 2.29357200 \\ \mathrm{C} & 1.29320200 & -3.34787300 & 1.88046600 \\ \mathrm{H} & 0.01122000 & -3.52677200 & 0.16273600 \\ \mathrm{C} & 1.05579900 & -1.33194500 & 3.16023900 \\ \mathrm{H} & -0.43962200 & 0.04843700 & 2.46810200 \\ \mathrm{C} & 1.67809600 & -2.55863400 & 2.95742000 \\ \mathrm{H} & 2.43438700 & -2.90887300 & 3.64952700 \\ \mathrm{H} & -3.41457300 & -2.55253500 & 0.14854500 \\ \mathrm{C} & -4.03195500 & -0.94238200 & -1.16128700 \\ \mathrm{C} & -4.25694700 & -2.29470200 & -0.49484500 \\ \mathrm{C} & -5.54963600 & -2.21169700 & 0.32301900 \\ \mathrm{C} & -5.45784500 & -1.10724400 & 1.39773200 \\ \mathrm{C} & -4.31383600 & -0.14551900 & 1.09074100 \\ \mathrm{H} & -6.38097500 & -1.99987500 & -0.35603100 \\ \mathrm{H} & -5.75850500 & -3.18091700 & 0.77815800 \\ \mathrm{H} & -6.40230800 & -0.56232500 & 1.45279700 \\ \mathrm{H} & -5.28024200 & -1.54035800 & 2.38583800 \\ \mathrm{H} & -4.45539300 & 0.82393200 & 1.56767900 \\ \mathrm{H} & -3.35146800 & -0.55375300 & 1.40625600 \\ \mathrm{O} & -4.25489700 & 0.14658600 & -0.31488800 \\ \mathrm{H} & -4.72231500 & -0.83381400 & -2.00435300 \\ \mathrm{H} & -4.33048500 & -3.05648800 & -1.27291500 \\ \mathrm{C} & -5.05365100 & 3.45670200 & -1.11621500 \\ \mathrm{H} & -5.79290500 & 3.26819700 & -0.32771100 \\ \mathrm{H} & -5.45440600 & 3.08127700 & -2.06593100 \\ \mathrm{H} & -4.92002900 & 4.53512200 & -1.20755600 \\ \mathrm{O} & -3.79430100 & 2.89402700 & -0.81205900 \\ \mathrm{H} & -3.90216200 & 1.93651500 & -0.74464500 \\ \mathrm{H} & 1.32514700 & -0.72264400 & 4.01592600 \\ \mathrm{H} & 1.74953700 & -4.31935400 & 1.72809900 \\ \mathrm{C} & 1.09423600 & -0.22996600 & -0.96918300 \\ \mathrm{C} & 1.41771000 & 0.86906500 & -0.21038800 \\ \mathrm{C} & 2.41900300 & 0.01365300 & -0.71148100 \\ \mathrm{~N} & 1.08494200 & 1.93202800 & 0.48559100\end{array}$

$\begin{array}{lrrr}\mathrm{N} & 3.68262800 & -0.30148500 & -0.87355800 \\ \mathrm{C} & 2.11200300 & 2.56330600 & 1.34389700 \\ \mathrm{C} & -0.20375600 & 2.64457500 & 0.23268300 \\ \mathrm{C} & 4.74950100 & 0.57866600 & -0.35117900 \\ \mathrm{C} & 4.03147400 & -1.56335200 & -1.56600300 \\ \mathrm{C} & 2.75496400 & 3.76313700 & 0.65292800 \\ \mathrm{H} & 2.86931300 & 1.79030600 & 1.49788600 \\ \mathrm{C} & 1.54683300 & 2.90685600 & 2.71771200 \\ \mathrm{C} & -1.36136100 & 2.12088300 & 1.07147400 \\ \mathrm{H} & -0.00118400 & 3.67263400 & 0.54062400 \\ \mathrm{C} & -0.53768800 & 2.67368600 & -1.25363500 \\ \mathrm{H} & 4.23866600 & 1.48268500 & -0.01831200 \\ \mathrm{C} & 5.43209700 & -0.06600100 & 0.85132200 \\ \mathrm{C} & 5.72712200 & 0.98738200 & -1.44865300 \\ \mathrm{H} & 5.11157300 & -1.66308700 & -1.45025900 \\ \mathrm{C} & 3.37461400 & -2.76333500 & -0.89586800 \\ \mathrm{C} & 3.71244000 & -1.46918100 & -3.05515700 \\ \mathrm{H} & 3.15803200 & 3.48868800 & -0.32566000 \\ \mathrm{H} & 2.03475200 & 4.57152200 & 0.50563700 \\ \mathrm{H} & 3.57124300 & 4.15498500 & 1.26376000 \\ \mathrm{H} & 0.78076200 & 3.68360100 & 2.66289900 \\ \mathrm{H} & 1.11557900 & 2.02271300 & 3.19085400 \\ \mathrm{H} & 2.34998100 & 3.28075300 & 3.35577300 \\ \mathrm{H} & -1.60441700 & 1.09132100 & 0.80351500 \\ \mathrm{H} & -1.13993100 & 2.16946700 & 2.13943100 \\ \mathrm{H} & -2.24478000 & 2.72737400 & 0.86310600 \\ \mathrm{H} & 0.26733800 & 3.13508000 & -1.83119200 \\ \mathrm{H} & -0.72695300 & 1.67258900 & -1.64761600 \\ \mathrm{H} & -1.46278400 & 3.23375000 & -1.39880900 \\ \mathrm{H} & 5.95542500 & -0.98354900 & 0.56920400 \\ \mathrm{H} & 6.17053700 & 0.61817100 & 1.27440000 \\ \mathrm{H} & 4.70163300 & -0.31328400 & 1.62555100 \\ \mathrm{H} & 5.20464400 & 1.45227400 & -2.28735000 \\ \mathrm{H} & 6.43985300 & 1.71097200 & -1.04811000 \\ \mathrm{H} & 6.30305600 & 0.13827400 & -1.82463000 \\ \mathrm{H} & 3.74944600 & -3.68609800 & -1.34331900 \\ \mathrm{H} & 3.58931500 & -2.78115800 & 0.17411800 \\ \mathrm{H} & 2.28855700 & -2.74744800 & -1.01822700 \\ & 2.63788800 & -1.34034500 & -3.21953800 \\ \mathrm{H} & 4.22866600 & -0.62710100 & -3.51906600 \\ & 4.01744500 & -2.38499400 & -3.56503700\end{array}$




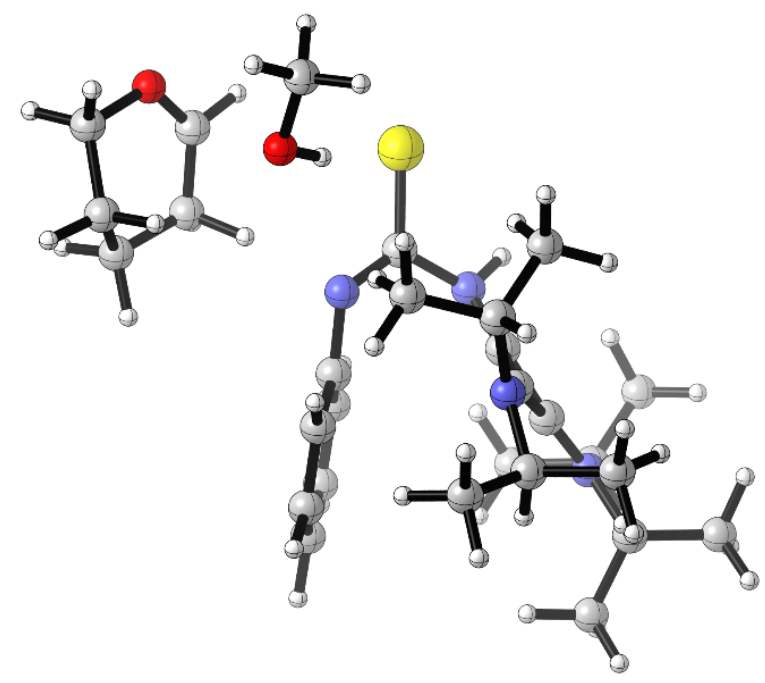

Figure S16. Optimized Structure of $E, E-\mathbf{1}-A n-\mathbf{T S}_{2}$.

- Thermochemistry -

$(1$ imaginary frequency $=-59.09 \mathrm{~Hz})$

Zero-point correction $=$

Thermal correction to Energy=

0.724296 (Hartree/Particle)

Thermal correction to Enthalpy=

0.765235

Thermal correction to Gibbs Free Energy=

0.766179

Sum of electronic and zero-point Energies=

0.648126

Sum of electronic and thermal Energies=

$-1861.848532$

Sum of electronic and thermal Enthalpies=

$-1861.807593$

Sum of electronic and thermal Free Energies=

$-1861.806649$

$-1861.924702$

$\begin{array}{lrrr}\mathrm{C} & -1.04354000 & -0.62884500 & -1.35539400 \\ \mathrm{~S} & -2.11888000 & -0.37418600 & -2.66295200 \\ \mathrm{~N} & 0.32075400 & -0.85995400 & -1.71305000 \\ \mathrm{H} & 0.48344400 & -1.16742700 & -2.66086000 \\ \mathrm{~N} & -1.38137400 & -0.60392300 & -0.10641600 \\ \mathrm{C} & -0.57507100 & -1.10668000 & 0.92920000 \\ \mathrm{C} & -0.08506700 & -2.41745100 & 0.89776700 \\ \mathrm{C} & -0.34681500 & -0.33705000 & 2.07435200 \\ \mathrm{C} & 0.65037800 & -2.92288900 & 1.96066600 \\ \mathrm{H} & -0.28994700 & -3.03510200 & 0.03025400 \\ \mathrm{C} & 0.40089600 & -0.84367500 & 3.12867900 \\ \mathrm{H} & -0.76413900 & 0.65994000 & 2.12535500 \\ \mathrm{C} & 0.91216600 & -2.13617300 & 3.07633900 \\ \mathrm{H} & 1.48261200 & -2.53457400 & 3.90687400 \\ \mathrm{H} & -3.18174800 & -1.15143600 & 0.06541900 \\ \mathrm{C} & -5.01277300 & -1.17531300 & -0.81686700 \\ \mathrm{C} & -4.15111200 & -1.70791100 & 0.21372400 \\ \mathrm{C} & -4.67195300 & -1.53973100 & 1.63456600 \\ \mathrm{C} & -5.37385500 & -0.18651600 & 1.73205200 \\ \mathrm{C} & -6.49596400 & -0.11034900 & 0.72212700 \\ \mathrm{H} & -5.36424700 & -2.34789300 & 1.89042300 \\ \mathrm{H} & -3.83752600 & -1.59201700 & 2.33431000 \\ \mathrm{H} & -5.80816500 & -0.03155900 & 2.72198400\end{array}$

$\begin{array}{lrrr}\mathrm{H} & -4.66078400 & 0.61537300 & 1.53127500 \\ \mathrm{H} & -7.32713200 & -0.77920300 & 0.95084100 \\ \mathrm{H} & -6.87228700 & 0.89795900 & 0.56781900 \\ \mathrm{O} & -6.06863900 & -0.51511100 & -0.63358300 \\ \mathrm{H} & -4.77521100 & -1.33452500 & -1.86727400 \\ \mathrm{H} & -3.87813700 & -2.73043100 & -0.07073600 \\ \mathrm{C} & -4.45582900 & 2.40771700 & -1.41529600 \\ \mathrm{H} & -4.90078400 & 1.99805800 & -2.33016400 \\ \mathrm{H} & -3.70930400 & 3.15814800 & -1.69995800 \\ \mathrm{H} & -5.23857100 & 2.90649800 & -0.84074200 \\ \mathrm{O} & -3.89853400 & 1.39701200 & -0.59939300 \\ \mathrm{H} & -3.22114500 & 0.94284300 & -1.12834800 \\ \mathrm{H} & 0.57029200 & -0.23044800 & 4.00735200 \\ \mathrm{H} & 1.01668900 & -3.94283100 & 1.91778500 \\ \mathrm{C} & 1.31303500 & -0.22334800 & -1.05741800 \\ \mathrm{C} & 1.71562500 & 0.84572800 & -0.29536900 \\ \mathrm{C} & 2.61375500 & -0.18743300 & -0.61265900 \\ \mathrm{~N} & 1.47360500 & 1.99940100 & 0.29499200 \\ \mathrm{~N} & 3.81224100 & -0.72368700 & -0.57051800 \\ \mathrm{C} & 2.45385200 & 2.50051100 & 1.28114200 \\ \mathrm{C} & 0.43924000 & 2.92651200 & -0.24390400 \\ \mathrm{C} & 4.92825600 & -0.01920600 & 0.09191400 \\ \mathrm{C} & 4.03522800 & -2.04737600 & -1.19592900\end{array}$




$\begin{array}{lrrrrrrr}\mathrm{C} & 3.44316600 & 3.47824800 & 0.64903900 & \mathrm{H} & -1.36085700 & 1.78405500 & 0.18068500 \\ \mathrm{H} & 2.99391900 & 1.61224900 & 1.61874900 & \mathrm{H} & -0.86812300 & 2.87538000 & 1.49282500 \\ \mathrm{C} & 1.75846500 & 3.08519600 & 2.50568100 & \mathrm{H} & -1.60574700 & 3.52374800 & 0.02712500 \\ \mathrm{C} & -0.92637400 & 2.75788900 & 0.40929800 & \mathrm{H} & 1.33954400 & 2.93898700 & -2.22667100 \\ \mathrm{H} & 0.82042600 & 3.91920000 & 0.00738900 & \mathrm{H} & -0.11560300 & 1.91778800 & -2.10430400 \\ \mathrm{C} & 0.35323000 & 2.84505900 & -1.76522800 & \mathrm{H} & -0.26827400 & 3.66467100 & -2.13092400 \\ \mathrm{H} & 4.52690900 & 0.95574200 & 0.36873500 & \mathrm{H} & 5.75565800 & -1.73659800 & 1.14768000 \\ \mathrm{C} & 5.34213800 & -0.74847400 & 1.36655300 & \mathrm{H} & 6.11308500 & -0.17903800 & 1.89020200 \\ \mathrm{C} & 6.09011400 & 0.21972500 & -0.86780600 & \mathrm{H} & 4.48766000 & -0.87417900 & 2.03584100 \\ \mathrm{H} & 5.06286500 & -2.31285000 & -0.94294400 & \mathrm{H} & 5.75638100 & 0.75207000 & -1.76111500 \\ \mathrm{C} & 3.11151000 & -3.10399500 & -0.60379300 & \mathrm{H} & 6.85266600 & 0.82508200 & -0.37350800 \\ \mathrm{C} & 3.92536600 & -1.95233500 & -2.71511300 & \mathrm{H} & 6.56545000 & -0.71420800 & -1.17774100 \\ \mathrm{H} & 3.91707800 & 3.04704500 & -0.23690400 & \mathrm{H} & 3.36887100 & -4.08607100 & -1.00607200 \\ \mathrm{H} & 2.95146200 & 4.40688500 & 0.34896300 & \mathrm{H} & 3.20212400 & -3.13581900 & 0.48350800 \\ \mathrm{H} & 4.22519700 & 3.73755600 & 1.36625400 & \mathrm{H} & 2.06639000 & -2.90133000 & -0.85041400 \\ \mathrm{H} & 1.20016500 & 3.99269200 & 2.26335700 & \mathrm{H} & 2.91085300 & -1.67844600 & -3.01952000 \\ \mathrm{H} & 1.07331900 & 2.35744400 & 2.94407300 & \mathrm{H} & 4.61669500 & -1.20848000 & -3.11511500 \\ \mathrm{H} & 2.50711800 & 3.35049200 & 3.25478100 & \mathrm{H} & 4.15532800 & -2.91754600 & -3.17050100\end{array}$

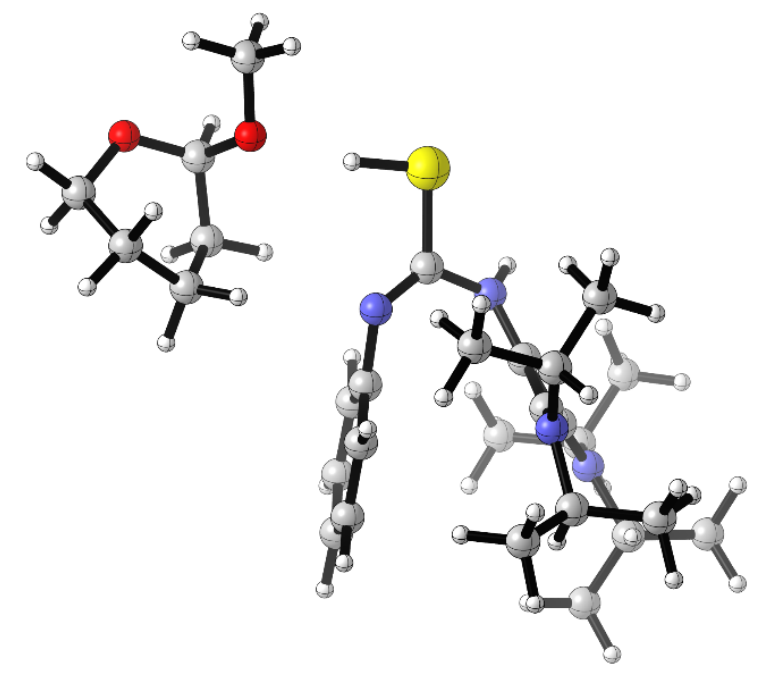

Figure S17. Optimized Structure of $E, E-1-A n-I_{N T}$.

- Thermochemistry -

(0 imaginary frequencies)

Zero-point correction $=$

Thermal correction to Energy=

0.726287 (Hartree/Particle)

Thermal correction to Enthalpy=

0.766382

Thermal correction to Gibbs Free Energy=

0.767326

Sum of electronic and zero-point Energies=

0.651748

Sum of electronic and thermal Energies=

$-1861.876862$

Sum of electronic and thermal Enthalpies=

$-1861.836766$

$-1861.835822$ 
Sum of electronic and thermal Free Energies=

$\begin{array}{lrrr}\mathrm{C} & -1.02379400 & -0.04235100 & -1.28245700 \\ \mathrm{~S} & -2.10352300 & 0.29164000 & -2.62558800 \\ \mathrm{~N} & 0.23163100 & -0.53994200 & -1.71702900 \\ \mathrm{H} & 0.26205100 & -1.22310800 & -2.45833500 \\ \mathrm{~N} & -1.33346200 & 0.17744800 & -0.07597800 \\ \mathrm{C} & -0.61826900 & -0.35278600 & 1.01486100 \\ \mathrm{C} & -0.45342200 & -1.73267700 & 1.15197900 \\ \mathrm{C} & -0.17221300 & 0.48913200 & 2.03434000 \\ \mathrm{C} & 0.18958700 & -2.25337000 & 2.26685700 \\ \mathrm{H} & -0.85475200 & -2.39490900 & 0.39258700 \\ \mathrm{C} & 0.48951900 & -0.03664800 & 3.13465000 \\ \mathrm{H} & -0.35664900 & 1.55162300 & 1.95805800 \\ \mathrm{C} & 0.68132000 & -1.40903000 & 3.25464900 \\ \mathrm{H} & 1.17557600 & -1.81859800 & 4.12737400 \\ \mathrm{H} & -3.36285400 & -1.47128200 & 0.03958900 \\ \mathrm{C} & -5.37515800 & -0.94526100 & -0.48859300 \\ \mathrm{C} & -4.32009800 & -1.30847900 & 0.53929600 \\ \mathrm{C} & -4.19943900 & -0.22366200 & 1.62287600 \\ \mathrm{C} & -5.54670500 & 0.48928700 & 1.83627500 \\ \mathrm{C} & -6.70150900 & -0.41668100 & 1.44268300 \\ \mathrm{H} & -3.85657700 & -0.68748000 & 2.55163700 \\ \mathrm{H} & -3.44107100 & 0.50043700 & 1.32539300 \\ \mathrm{H} & -5.67049000 & 0.79712900 & 2.87741200 \\ \mathrm{H} & -5.59105200 & 1.39077700 & 1.22016500 \\ \mathrm{H} & -6.70869200 & -1.32107200 & 2.06578700 \\ \mathrm{H} & -7.66328800 & 0.08166500 & 1.56662100 \\ \mathrm{O} & -6.64412500 & -0.80516200 & 0.06746500 \\ \mathrm{H} & -5.47829300 & -1.71410400 & -1.26504800 \\ \mathrm{H} & -4.62152800 & -2.26385000 & 0.97848500 \\ \mathrm{C} & -5.86326300 & 0.73511400 & -2.10071500 \\ \mathrm{H} & -6.01183900 & -0.01376500 & -2.88962000 \\ \mathrm{H} & -5.42701800 & 1.63673600 & -2.53127800 \\ \mathrm{H} & -6.82880600 & 0.97286800 & -1.65170200 \\ \mathrm{O} & -4.95412000 & 0.26385400 & -1.11882500 \\ \mathrm{H} & -3.24921500 & 0.30280300 & -1.87327600 \\ \mathrm{H} & 0.83168200 & 0.62889000 & 3.91952200 \\ \mathrm{H} & 0.29557000 & -3.32747300 & 2.36876200 \\ \mathrm{C} & 1.37088100 & -0.17988200 & -1.08682200 \\ \mathrm{C} & 2.06780300 & 0.80134000 & -0.42980900 \\ \mathrm{~N} & 2.62729200 & -0.47662200 & -0.61733600 \\ & 2.16503200 & 2.02939600 & 0.03279800\end{array}$

$-1861.951400$

$\begin{array}{lrrr}\mathrm{N} & 3.62386100 & -1.32092300 & -0.49894500 \\ \mathrm{C} & 3.24596600 & 2.33968800 & 0.99498200 \\ \mathrm{C} & 1.41090300 & 3.14130800 & -0.61082500 \\ \mathrm{C} & 4.89356100 & -0.89714300 & 0.12997900 \\ \mathrm{C} & 3.47396700 & -2.70347800 & -1.00946800 \\ \mathrm{C} & 4.46495000 & 2.93716500 & 0.29623800 \\ \mathrm{H} & 3.51801100 & 1.37650900 & 1.43419000 \\ \mathrm{C} & 2.73471900 & 3.21426100 & 2.13457200 \\ \mathrm{C} & 0.03656600 & 3.37876500 & 0.00278900 \\ \mathrm{H} & 2.02610600 & 4.02328500 & -0.41993200 \\ \mathrm{C} & 1.33660900 & 2.95628600 & -2.12341900 \\ \mathrm{H} & 4.77831800 & 0.17151500 & 0.31131900 \\ \mathrm{C} & 5.08420600 & -1.59676500 & 1.47190400 \\ \mathrm{C} & 6.07609000 & -1.07719900 & -0.81670600 \\ \mathrm{H} & 4.39372800 & -3.21356400 & -0.72026600 \\ \mathrm{C} & 2.30887100 & -3.42161300 & -0.34175000 \\ \mathrm{C} & 3.38332200 & -2.71013900 & -2.53264300 \\ \mathrm{H} & 4.81517900 & 2.29215000 & -0.51431000 \\ \mathrm{H} & 4.24316700 & 3.91982400 & -0.12729200 \\ \mathrm{H} & 5.28091200 & 3.06408800 & 1.01117700 \\ \mathrm{H} & 2.45052400 & 4.21147700 & 1.79033700 \\ \mathrm{H} & 1.87438500 & 2.75121600 & 2.62073000 \\ \mathrm{H} & 3.52463700 & 3.33702900 & 2.87808000 \\ \mathrm{H} & -0.62890800 & 2.52967300 & -0.16445900 \\ \mathrm{H} & 0.10579700 & 3.56827200 & 1.07482200 \\ \mathrm{H} & -0.41642400 & 4.25723400 & -0.46238300 \\ \mathrm{H} & 2.32475000 & 2.77211000 & -2.55178600 \\ \mathrm{H} & 0.66900400 & 2.13697500 & -2.40231500 \\ \mathrm{H} & 0.93411800 & 3.86345000 & -2.57713300 \\ \mathrm{H} & 5.20606100 & -2.67604300 & 1.34675000 \\ \mathrm{H} & 5.98299600 & -1.21979500 & 1.96413300 \\ \mathrm{H} & 4.22788300 & -1.41984700 & 2.12721200 \\ \mathrm{H} & 5.90528600 & -0.55851400 & -1.76249500 \\ \mathrm{H} & 6.97488800 & -0.66159100 & -0.35696100 \\ \mathrm{H} & 6.27629900 & -2.13042800 & -1.02840600 \\ \mathrm{H} & 2.29006500 & -4.46724900 & -0.65542300 \\ \mathrm{H} & 2.40044800 & -3.38278500 & 0.74477700 \\ \mathrm{H} & 1.35120600 & -2.97066800 & -0.61376900 \\ \mathrm{H} & 2.48689300 & -2.18661900 & -2.87983200 \\ & 4.25284200 & -2.22810500 & -2.98266600 \\ & 3.32838700 & -3.73585200 & -2.90219200\end{array}$




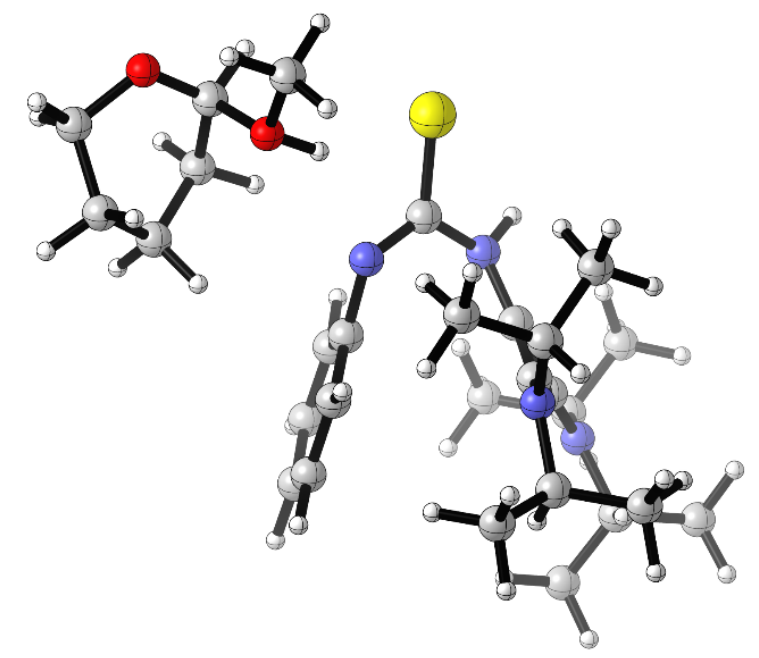

Figure S18. Optimized Structure of E,E-1-An-TS 3 -PA.

- Thermochemistry -

$(1$ imaginary frequency $=-317.72 \mathrm{~Hz})$

Zero-point correction=

0.728079 (Hartree/Particle)

Thermal correction to Energy=

0.767512

Thermal correction to Enthalpy=

0.768456

Thermal correction to Gibbs Free Energy $=0.654958$

Sum of electronic and zero-point Energies $=$

$-1861.859264$

Sum of electronic and thermal Energies $=$

$-1861.819831$

Sum of electronic and thermal Enthalpies=

$-1861.818887$

Sum of electronic and thermal Free Energies=

$-1861.932384$

$\begin{array}{lrrr}\mathrm{C} & -1.02513900 & -0.07878800 & -1.49616200 \\ \mathrm{~S} & -2.03548400 & 0.34850800 & -2.80577500 \\ \mathrm{~N} & 0.28796000 & -0.52910000 & -1.82186500 \\ \mathrm{H} & 0.42724000 & -0.89749400 & -2.75058200 \\ \mathrm{~N} & -1.40289200 & 0.00230900 & -0.26087300 \\ \mathrm{C} & -0.74871100 & -0.65644500 & 0.79309100 \\ \mathrm{C} & -0.49908700 & -2.03319100 & 0.74339900 \\ \mathrm{C} & -0.44930000 & 0.03024100 & 1.97390600 \\ \mathrm{C} & 0.06791800 & -2.69008900 & 1.82646900 \\ \mathrm{H} & -0.76361400 & -2.58481700 & -0.15231200 \\ \mathrm{C} & 0.12974100 & -0.62933200 & 3.04940100 \\ \mathrm{H} & -0.68222300 & 1.08468300 & 2.04075200 \\ \mathrm{C} & 0.39931300 & -1.99200600 & 2.98177700 \\ \mathrm{H} & 0.83689000 & -2.50721400 & 3.82844200 \\ \mathrm{H} & -3.28809500 & -1.74646000 & -0.50513700 \\ \mathrm{C} & -5.03058300 & -0.55118100 & -0.79995300 \\ \mathrm{C} & -4.27737600 & -1.62713200 & -0.06235900 \\ \mathrm{C} & -4.20121700 & -1.34962300 & 1.44948400 \\ \mathrm{C} & -5.37735100 & -0.47378400 & 1.91535000 \\ \mathrm{C} & -6.57805900 & -0.65281700 & 1.00614100 \\ \mathrm{H} & -4.20211500 & -2.30433400 & 1.97885600 \\ \mathrm{H} & -3.25738400 & -0.85781000 & 1.68417100 \\ \mathrm{H} & -5.66866500 & -0.71873900 & 2.93870300\end{array}$

$\begin{array}{lrrr}\mathrm{H} & -5.08937800 & 0.58085000 & 1.90980100 \\ \mathrm{H} & -6.92630900 & -1.69075900 & 1.00452400 \\ \mathrm{H} & -7.41097700 & -0.01086900 & 1.28688100 \\ \mathrm{O} & -6.27542500 & -0.28842000 & -0.36179900 \\ \mathrm{H} & -5.03937100 & -0.66265200 & -1.88467600 \\ \mathrm{H} & -4.83247100 & -2.54872300 & -0.26450600 \\ \mathrm{C} & -4.75950900 & 1.93465700 & -1.22171900 \\ \mathrm{H} & -4.81383000 & 1.78650900 & -2.30047400 \\ \mathrm{H} & -4.09147000 & 2.75645600 & -0.97742100 \\ \mathrm{H} & -5.74348800 & 2.07947600 & -0.78706600 \\ \mathrm{O} & -4.19557800 & 0.75371100 & -0.59635900 \\ \mathrm{H} & -3.27844500 & 0.58849600 & -0.98610800 \\ \mathrm{H} & 0.35394800 & -0.07854100 & 3.95666600 \\ \mathrm{H} & 0.24360800 & -3.75872800 & 1.76804200 \\ \mathrm{C} & 1.35289100 & -0.10716600 & -1.11265600 \\ \mathrm{C} & 1.90948600 & 0.85116500 & -0.30301600 \\ \mathrm{C} & 2.61848900 & -0.31981000 & -0.61754800 \\ \mathrm{~N} & 1.85303100 & 2.01649500 & 0.31157100 \\ \mathrm{~N} & 3.69778100 & -1.06448900 & -0.54368300 \\ \mathrm{C} & 2.85664000 & 2.30836600 & 1.35680900 \\ \mathrm{C} & 1.02805700 & 3.12459000 & -0.24305400 \\ \mathrm{C} & 4.88651100 & -0.58650700 & 0.19142100 \\ \mathrm{C} & 3.70143800 & -2.40278000 & -1.17732000\end{array}$




$\begin{array}{lrrrrrrr}\mathrm{C} & 4.02423400 & 3.12749500 & 0.80931100 & \mathrm{H} & -0.95306200 & 2.30039000 & 0.06753100 \\ \mathrm{H} & 3.22623100 & 1.33023200 & 1.67516400 & \mathrm{H} & -0.35665200 & 3.27753000 & 1.42874600 \\ \mathrm{C} & 2.21020000 & 2.95505200 & 2.57687700 & \mathrm{H} & -0.88819400 & 4.06899000 & -0.05756000 \\ \mathrm{C} & -0.37861300 & 3.18816100 & 0.34130300 & \mathrm{H} & 2.01063300 & 3.00516100 & -2.18245900 \\ \mathrm{H} & 1.56353200 & 4.02843900 & 0.05718500 & \mathrm{H} & 0.38359500 & 2.27915700 & -2.15340400 \\ \mathrm{C} & 1.00307500 & 3.09345600 & -1.76865700 & \mathrm{H} & 0.56733700 & 4.02328300 & -2.13874600 \\ \mathrm{H} & 4.67735500 & 0.45777200 & 0.42535000 & \mathrm{H} & 5.25450300 & -2.41273600 & 1.31656400 \\ \mathrm{C} & 5.04989800 & -1.35431500 & 1.49967700 & \mathrm{H} & 5.88777800 & -0.94982600 & 2.07141400 \\ \mathrm{C} & 6.13825900 & -0.61454800 & -0.68043400 & \mathrm{H} & 4.14445100 & -1.28154600 & 2.10733900 \\ \mathrm{H} & 4.64213400 & -2.86188400 & -0.86962400 & \mathrm{H} & 5.98286800 & -0.05992600 & -1.60818600 \\ \mathrm{C} & 2.56247000 & -3.27089600 & -0.65764600 & \mathrm{H} & 6.96731900 & -0.15241500 & -0.14081200 \\ \mathrm{C} & 3.70140800 & -2.27802500 & -2.69823800 & \mathrm{H} & 6.44157600 & -1.63389800 & -0.93123800 \\ \mathrm{H} & 4.45739200 & 2.65960100 & -0.07888900 & \mathrm{H} & 2.65530500 & -4.27980500 & -1.06505800 \\ \mathrm{H} & 3.71394200 & 4.13984200 & 0.53910100 & \mathrm{H} & 2.58205700 & -3.32969500 & 0.43210300 \\ \mathrm{H} & 4.80647400 & 3.21645200 & 1.56664900 & \mathrm{H} & 1.58961900 & -2.87366000 & -0.95732100 \\ \mathrm{H} & 1.81654400 & 3.94932400 & 2.35180500 & \mathrm{H} & 2.77852200 & -1.81044200 & -3.05459100 \\ \mathrm{H} & 1.39810000 & 2.33241700 & 2.95654800 & \mathrm{H} & 4.54438600 & -1.67803100 & -3.04555200 \\ \mathrm{H} & 2.95695200 & 3.06824200 & 3.36539500 & \mathrm{H} & 3.76955200 & -3.26573600 & -3.15832200\end{array}$

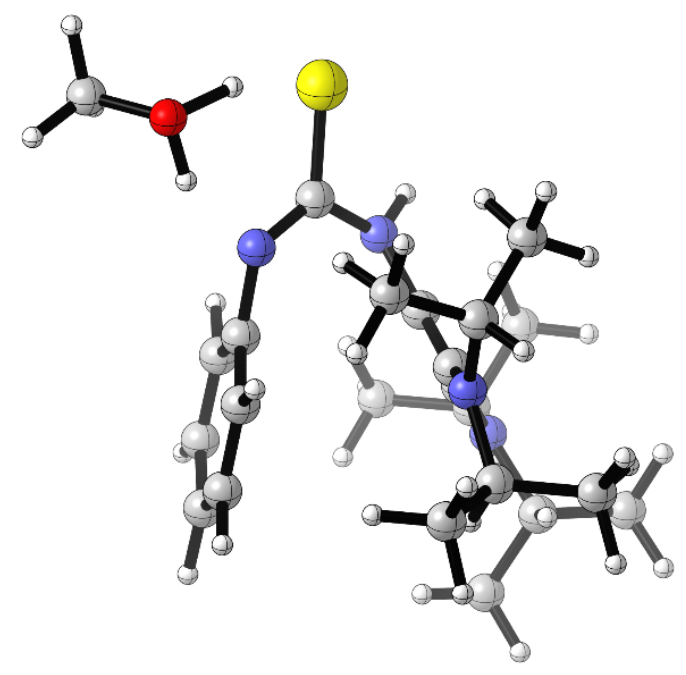

Figure S19. Optimized Structure of E,E-1-An-TS 3 -MA.

- Thermochemistry -

$(1$ imaginary frequency $=-707.17 \mathrm{~Hz})$

Zero-point correction=

Thermal correction to Energy=

Thermal correction to Enthalpy=

Thermal correction to Gibbs Free Energy=

Sum of electronic and zero-point Energies=

Sum of electronic and thermal Energies=

Sum of electronic and thermal Enthalpies=
0.596566 (Hartree/Particle)

0.630160

0.631104

0.531491

$-1591.442070$

$-1591.408476$

$-1591.407532$ 
Sum of electronic and thermal Free Energies=

$\begin{array}{lrrr}\mathrm{C} & -2.26647700 & -0.66928200 & -0.93660700 \\ \mathrm{~S} & -3.46826900 & -0.62304100 & -2.18533000 \\ \mathrm{~N} & -0.97781600 & -1.04482600 & -1.37376600 \\ \mathrm{H} & -0.91740200 & -1.59539800 & -2.21720700 \\ \mathrm{~N} & -2.50435100 & -0.36939000 & 0.29683400 \\ \mathrm{C} & -1.59845600 & -0.65107000 & 1.34855200 \\ \mathrm{C} & -1.15720500 & -1.95482400 & 1.58184300 \\ \mathrm{C} & -1.21575000 & 0.36257300 & 2.22687100 \\ \mathrm{C} & -0.31120200 & -2.22702000 & 2.64792700 \\ \mathrm{H} & -1.49343500 & -2.75334700 & 0.93002400 \\ \mathrm{C} & -0.35798900 & 0.08645200 & 3.28341000 \\ \mathrm{H} & -1.59792400 & 1.36349300 & 2.07759800 \\ \mathrm{C} & 0.10645600 & -1.20677500 & 3.49444000 \\ \mathrm{H} & 0.76077400 & -1.42395100 & 4.33023300 \\ \mathrm{H} & -0.06927700 & 0.88266200 & 3.96060000 \\ \mathrm{H} & 0.01212100 & -3.24659300 & 2.82456500 \\ \mathrm{C} & 0.12701900 & -0.39403500 & -0.93423100 \\ \mathrm{C} & 0.68955700 & 0.76916400 & -0.47477900 \\ \mathrm{C} & 1.45949300 & -0.40587100 & -0.60032900 \\ \mathrm{~N} & 0.60645500 & 2.04475400 & -0.16509500 \\ \mathrm{~N} & 2.60692400 & -1.03444600 & -0.52820800 \\ \mathrm{C} & 1.71037400 & 2.66023800 & 0.60550600 \\ \mathrm{C} & -0.39651900 & 2.92001600 & -0.83509700 \\ \mathrm{C} & 3.83379100 & -0.31935800 & -0.11513100 \\ \mathrm{C} & 2.67747400 & -2.47010500 & -0.89004900 \\ \mathrm{C} & 2.71715100 & 3.34902900 & -0.31293600 \\ \mathrm{H} & 2.19830500 & 1.82463400 & 1.11381400 \\ \mathrm{C} & 1.17379600 & 3.58531700 & 1.69195900 \\ \mathrm{C} & -1.72223000 & 3.00215100 & -0.08937100 \\ \mathrm{H} & 0.06617400 & 3.90898400 & -0.82027100 \\ \mathrm{C} & -0.58806900 & 2.52834000 & -2.29722400 \\ \mathrm{H} & 3.53364400 & 0.72163200 & 0.00384700 \\ \mathrm{C} & 4.32318300 & -0.83553300 & 1.23441000 \\ \mathrm{C} & 4.90314300 & -0.36789800 & -1.20196100\end{array}$

$-1591.507145$

$\begin{array}{lrrr}\mathrm{H} & 3.70704800 & -2.76302400 & -0.68080800 \\ \mathrm{C} & 1.75850100 & -3.31305500 & -0.01644400 \\ \mathrm{C} & 2.41972600 & -2.66678300 & -2.38089400 \\ \mathrm{H} & 3.07905000 & 2.66760600 & -1.08772600 \\ \mathrm{H} & 2.27800700 & 4.21916500 & -0.80703200 \\ \mathrm{H} & 3.57558600 & 3.69826100 & 0.26512100 \\ \mathrm{H} & 0.67236100 & 4.46146200 & 1.27415200 \\ \mathrm{H} & 0.47404500 & 3.05435400 & 2.33976000 \\ \mathrm{H} & 2.00415500 & 3.94359800 & 2.30345600 \\ \mathrm{H} & -2.22460600 & 2.03332500 & -0.07653400 \\ \mathrm{H} & -1.58386700 & 3.34528900 & 0.93713800 \\ \mathrm{H} & -2.37615000 & 3.71489400 & -0.59668700 \\ \mathrm{H} & 0.37102600 & 2.44029500 & -2.81337500 \\ \mathrm{H} & -1.13950400 & 1.59048100 & -2.40246400 \\ \mathrm{H} & -1.17435100 & 3.29836600 & -2.80139200 \\ \mathrm{H} & 4.64025600 & -1.88000900 & 1.17405500 \\ \mathrm{H} & 5.18285900 & -0.25054700 & 1.56752200 \\ \mathrm{H} & 3.53595600 & -0.75781700 & 1.98809100 \\ \mathrm{H} & 4.51957400 & 0.02301100 & -2.14697300 \\ \mathrm{H} & 5.75533500 & 0.24434700 & -0.90017000 \\ \mathrm{H} & 5.27421300 & -1.38198300 & -1.36940600 \\ \mathrm{H} & 1.91890800 & -4.37218200 & -0.22789000 \\ \mathrm{H} & 1.95463500 & -3.13437600 & 1.04199600 \\ \mathrm{H} & 0.70703500 & -3.08609400 & -0.21002600 \\ \mathrm{H} & 1.39134200 & -2.39972200 & -2.64269900 \\ \mathrm{H} & 3.09664500 & -2.05964800 & -2.98441200 \\ \mathrm{H} & 2.56393600 & -3.71489800 & -2.64968000 \\ \mathrm{H} & -4.60197700 & -0.24767400 & -1.07109000 \\ \mathrm{O} & -5.08091000 & 0.01570600 & 0.01612700 \\ \mathrm{H} & -4.17991500 & -0.00089100 & 0.45895000 \\ \mathrm{C} & -5.88713100 & -1.06794800 & 0.51169900 \\ \mathrm{H} & -6.08819900 & -0.89940000 & 1.56856900 \\ \mathrm{H} & -6.82122000 & -1.05710300 & -0.04515400 \\ \mathrm{H} & -5.37588100 & -2.02316400 & 0.37002800 \\ & & & \\ & & & \\ & & & \end{array}$

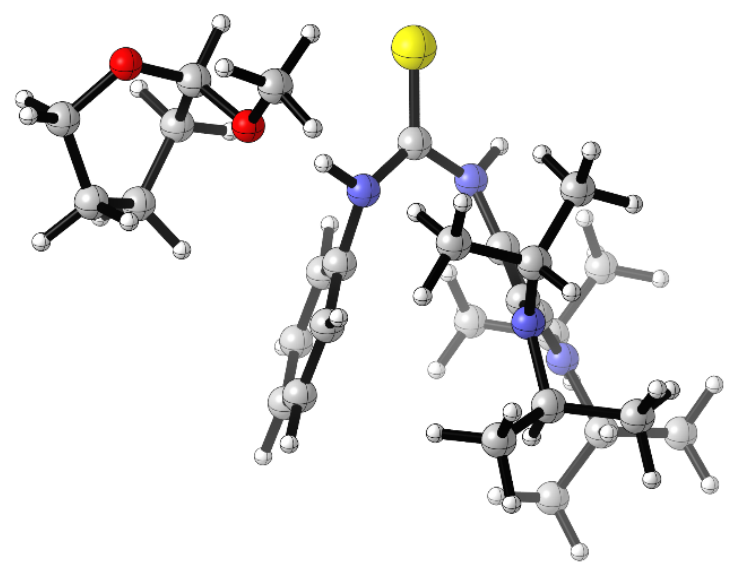

Figure S20. Optimized Structure of $E, E-\mathbf{1}-A n-I_{N} \mathbf{5}_{5}$ 
- Thermochemistry -

(0 imaginary frequencies)

Zero-point correction=

Thermal correction to Energy=

Thermal correction to Enthalpy=

Thermal correction to Gibbs Free Energy=

Sum of electronic and zero-point Energies=

Sum of electronic and thermal Energies=

Sum of electronic and thermal Enthalpies=

Sum of electronic and thermal Free Energies=
0.730400 (Hartree/Particle)

0.770169

0.771113

0.656411

$-1861.900976$

$-1861.861207$

$-1861.860263$

$-1861.974965$

$\begin{array}{lrrr}\mathrm{C} & -0.85985200 & -0.18939500 & -1.90299800 \\ \mathrm{~S} & -1.76340200 & 0.04318200 & -3.26558200 \\ \mathrm{~N} & 0.49086900 & -0.55828700 & -2.00174400 \\ \mathrm{H} & 0.77542600 & -0.91690000 & -2.90135100 \\ \mathrm{~N} & -1.31347600 & -0.04019400 & -0.64314400 \\ \mathrm{C} & -0.84683300 & -0.74481100 & 0.50573500 \\ \mathrm{C} & -0.60804400 & -2.11479700 & 0.44750900 \\ \mathrm{C} & -0.72801100 & -0.06830100 & 1.71572400 \\ \mathrm{C} & -0.22145700 & -2.79747300 & 1.59218100 \\ \mathrm{H} & -0.74915300 & -2.64334600 & -0.48837900 \\ \mathrm{C} & -0.34833500 & -0.75886100 & 2.85929700 \\ \mathrm{H} & -0.95108500 & 0.98975400 & 1.75841700 \\ \mathrm{C} & -0.08564600 & -2.12268800 & 2.79972800 \\ \mathrm{H} & 0.20102800 & -2.66122600 & 3.69503700 \\ \mathrm{H} & -3.42057700 & -1.78416700 & -0.71239800 \\ \mathrm{C} & -4.87211600 & -0.20157300 & -0.69886700 \\ \mathrm{C} & -4.37512500 & -1.55776200 & -0.23192800 \\ \mathrm{C} & -4.28359400 & -1.63458500 & 1.30396300 \\ \mathrm{C} & -5.28537400 & -0.66764900 & 1.95795500 \\ \mathrm{C} & -6.49029700 & -0.46088800 & 1.05387400 \\ \mathrm{H} & -4.48854900 & -2.66150400 & 1.61734100 \\ \mathrm{H} & -3.27185200 & -1.40203800 & 1.64169000 \\ \mathrm{H} & -5.62262700 & -1.04692300 & 2.92557200 \\ \mathrm{H} & -4.81081500 & 0.30133900 & 2.12980000 \\ \mathrm{H} & -7.00291800 & -1.41604300 & 0.87994100 \\ \mathrm{H} & -7.20926400 & 0.22944600 & 1.49571800 \\ \mathrm{O} & -6.13640100 & 0.10757200 & -0.21060600 \\ \mathrm{H} & -4.94542600 & -0.14751600 & -1.79016000 \\ \mathrm{H} & -5.09257200 & -2.29280300 & -0.60660300 \\ \mathrm{C} & -4.21012100 & 2.08326400 & -0.75799700 \\ \mathrm{H} & -4.11970100 & 2.11975300 & -1.85004000 \\ \mathrm{H} & -3.48429700 & 2.76192700 & -0.30941900 \\ \mathrm{H} & -5.21786900 & 2.37880200 & -0.46141700 \\ \mathrm{O} & -3.91637700 & 0.77962500 & -0.27645500 \\ \mathrm{H} & -2.28400600 & 0.31085500 & -0.56877100 \\ \mathrm{H} & -0.27024700 & -0.23207700 & 3.80334100 \\ \mathrm{C} & -0.04819300 & -3.86625400 & 1.54318200 \\ \mathrm{C} & 1.43949800 & -0.09842700 & -1.14793600 \\ \mathrm{~N} & 1.87891700 & 0.87785600 & -0.29067400 \\ & 2.63629800 & -0.29334600 & -0.50240200 \\ & 1.74102000 & 2.04290700 & 0.30450100\end{array}$

$\begin{array}{lrrr}\mathrm{N} & 3.70595400 & -1.02074600 & -0.29509000 \\ \mathrm{C} & 2.64837800 & 2.36679800 & 1.42957000 \\ \mathrm{C} & 0.95064300 & 3.13575500 & -0.32964000 \\ \mathrm{C} & 4.80384700 & -0.52360400 & 0.56301700 \\ \mathrm{C} & 3.80922400 & -2.35857900 & -0.92726000 \\ \mathrm{C} & 3.85694100 & 3.17433900 & 0.96034700 \\ \mathrm{H} & 2.98845500 & 1.39873200 & 1.80562100 \\ \mathrm{C} & 1.89864100 & 3.04502000 & 2.57036100 \\ \mathrm{C} & -0.49431900 & 3.18960200 & 0.15029600 \\ \mathrm{H} & 1.45456300 & 4.04849500 & -0.00417500 \\ \mathrm{C} & 1.03953600 & 3.08239100 & -1.85137400 \\ \mathrm{H} & 4.54656900 & 0.51144800 & 0.78797400 \\ \mathrm{C} & 4.85396300 & -1.30834300 & 1.87002700 \\ \mathrm{C} & 6.13518200 & -0.50905900 & -0.18172000 \\ \mathrm{H} & 4.71301200 & -2.80149000 & -0.50703100 \\ \mathrm{C} & 2.62925300 & -3.24445900 & -0.54893800 \\ \mathrm{C} & 3.99158600 & -2.22997200 & -2.43638600 \\ \mathrm{H} & 4.36121900 & 2.68579500 & 0.12207300 \\ \mathrm{H} & 3.57008400 & 4.17917700 & 0.64077400 \\ \mathrm{H} & 4.57493100 & 3.28294700 & 1.77623500 \\ \mathrm{H} & 1.52335200 & 4.03068300 & 2.28549900 \\ \mathrm{H} & 1.05961400 & 2.43112600 & 2.90341600 \\ \mathrm{H} & 2.57798100 & 3.18448600 & 3.41343400 \\ \mathrm{H} & -1.03935600 & 2.30725000 & -0.18585300 \\ \mathrm{H} & -0.56027300 & 3.25887100 & 1.23708200 \\ \mathrm{H} & -0.98034900 & 4.07156500 & -0.27335600 \\ \mathrm{H} & 2.07636700 & 3.01021200 & -2.18848100 \\ \mathrm{H} & 0.46864200 & 2.24893300 & -2.26862700 \\ \mathrm{H} & 0.61316500 & 3.99690000 & -2.26685400 \\ \mathrm{H} & 5.09700800 & -2.35988800 & 1.69601100 \\ \mathrm{H} & 5.62377800 & -0.89671600 & 2.52591800 \\ \mathrm{H} & 3.89332700 & -1.26023000 & 2.38867600 \\ \mathrm{H} & 6.05992100 & 0.05965100 & -1.11095900 \\ \mathrm{H} & 6.89443800 & -0.03824900 & 0.44577800 \\ \mathrm{H} & 6.48748100 & -1.51616500 & -0.41720500 \\ \mathrm{H} & 2.79391200 & -4.25570000 & -0.92603500 \\ \mathrm{H} & 2.50530900 & -3.29143100 & 0.53449600 \\ \mathrm{H} & 1.69747100 & -2.87383400 & -0.98322200 \\ & 3.11278000 & -1.77402600 & -2.90239500 \\ \mathrm{H} & 4.86302000 & -1.62023700 & -2.68055900 \\ & 4.12566200 & -3.21635800 & -2.88438300\end{array}$




\section{ii. E,Z Conformation}

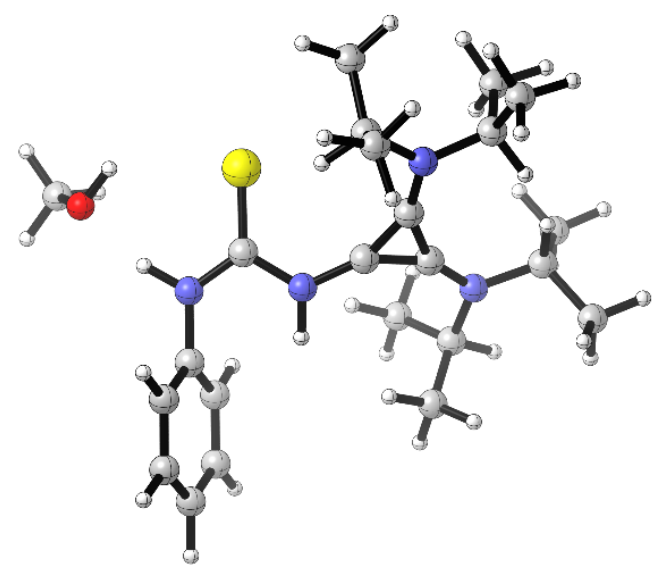

Figure S21. Optimized Structure of E,Z-1-An-INT 1.

- Thermochemistry -

(0 imaginary frequencies)

Zero-point correction $=$

Thermal correction to Energy=

Thermal correction to Enthalpy=

Thermal correction to Gibbs Free Energy=

Sum of electronic and zero-point Energies
Sum of electronic and thermal Energies=

Sum of electronic and thermal Enthalpies=

Sum of electronic and thermal Free Energies=
0.602056 (Hartree/Particle)

\subsection{7}

0.637792

$\begin{array}{lrrr}\mathrm{C} & -1.69895000 & -0.94482800 & 0.27324700 \\ \mathrm{~S} & -0.99553400 & -2.23085900 & 1.08507800 \\ \mathrm{~N} & -3.01681100 & -0.77676700 & 0.14122900 \\ \mathrm{H} & -3.60683100 & -1.53307000 & 0.52637900 \\ \mathrm{~N} & -0.93408200 & 0.06200400 & -0.29718200 \\ \mathrm{C} & -3.63938800 & 0.33865000 & -0.49887000 \\ \mathrm{C} & -4.22286400 & 0.17675200 & -1.75073700 \\ \mathrm{C} & -3.66561100 & 1.57787200 & 0.13902300 \\ \mathrm{C} & -4.83174600 & 1.26219200 & -2.36747600 \\ \mathrm{H} & -4.19858900 & -0.79593700 & -2.22742500 \\ \mathrm{C} & -4.26368500 & 2.66225700 & -0.49131600 \\ \mathrm{H} & -3.23739200 & 1.67713300 & 1.13100800 \\ \mathrm{C} & -4.84653900 & 2.50419000 & -1.74337300 \\ \mathrm{H} & -5.32212000 & 3.34808700 & -2.22849600 \\ \mathrm{H} & -1.42075300 & 0.89740400 & -0.59807400 \\ \mathrm{C} & -4.78758600 & -2.80617500 & 2.59601100 \\ \mathrm{H} & -4.94501500 & -3.77005200 & 3.08769600 \\ \mathrm{H} & -5.75495700 & -2.32220500 & 2.46552900 \\ \mathrm{H} & -4.15372000 & -2.17643200 & 3.23036000\end{array}$

$\begin{array}{lrrr}\mathrm{O} & -4.22801600 & -2.97858200 & 1.29934800 \\ \mathrm{H} & -3.35675700 & -3.37953400 & 1.39427400 \\ \mathrm{C} & 0.42453100 & 0.13319900 & -0.23865400 \\ \mathrm{C} & 1.67348900 & -0.41492700 & -0.39073400 \\ \mathrm{C} & 1.50506400 & 0.93744700 & -0.00112600 \\ \mathrm{~N} & 2.46169300 & -1.42407300 & -0.66745600 \\ \mathrm{~N} & 1.96052600 & 2.11884400 & 0.34699200 \\ \mathrm{C} & 3.91729000 & -1.23151800 & -0.42781500 \\ \mathrm{C} & 1.81081600 & -2.71318100 & -1.04282000 \\ \mathrm{C} & 3.40830700 & 2.38620300 & 0.48362800 \\ \mathrm{C} & 0.98217200 & 3.17044800 & 0.71614400 \\ \mathrm{C} & 4.25341100 & -1.41021200 & 1.05122800 \\ \mathrm{H} & 4.10185900 & -0.19008000 & -0.70196300 \\ \mathrm{C} & 4.81156100 & -2.06422400 & -1.33597200 \\ \mathrm{C} & 2.13394000 & -3.83331400 & -0.06223100 \\ \mathrm{H} & 3.90228200 & 1.47774800 & 0.14003600 \\ \mathrm{C} & 3.84429800 & 3.53455800 & -0.42056300 \\ \mathrm{C} & 3.78492200 & 2.60113500 & 1.94679200 \\ \mathrm{H} & 1.58620000 & 4.01518200 & 1.04944200\end{array}$




$\begin{array}{lrrrrrrr}\mathrm{C} & 0.17937600 & 3.62270100 & -0.49987500 & \mathrm{H} & 3.47894000 & 1.74967900 & 2.55872600 \\ \mathrm{C} & 0.10572400 & 2.73093400 & 1.88581400 & \mathrm{H} & 4.86679700 & 2.71680500 & 2.03702100 \\ \mathrm{H} & 3.61869900 & -0.77841600 & 1.67887300 & \mathrm{H} & -0.48608700 & 4.44293300 & -0.22388000 \\ \mathrm{H} & 4.11726300 & -2.44593500 & 1.36601500 & \mathrm{H} & 0.83860300 & 3.96640100 & -1.29885100 \\ \mathrm{H} & 5.29485500 & -1.13421000 & 1.23240300 & \mathrm{H} & -0.44472100 & 2.81779400 & -0.89867100 \\ \mathrm{H} & 4.73231600 & -3.13324100 & -1.13845100 & \mathrm{H} & -0.54895100 & 1.89919800 & 1.61183600 \\ \mathrm{H} & 4.59870700 & -1.88018400 & -2.38930100 & \mathrm{H} & 0.71507100 & 2.41971500 & 2.73639200 \\ \mathrm{H} & 5.84732600 & -1.77505500 & -1.14730700 & \mathrm{H} & -0.52749000 & 3.56173100 & 2.20372600 \\ \mathrm{H} & 3.17542800 & -4.15563700 & -0.11657700 & \mathrm{H} & -5.29514700 & 1.13674700 & -3.33858300 \\ \mathrm{H} & 1.90498700 & -3.52997500 & 0.96070000 & \mathrm{H} & -4.29317900 & 3.62488000 & 0.00520000 \\ \mathrm{H} & 1.50996200 & -4.69673000 & -0.30310200 & \mathrm{H} & 0.74765700 & -2.50373600 & -0.92336800 \\ \mathrm{H} & 3.38519100 & 4.48154600 & -0.12566600 & \mathrm{C} & 2.04426000 & -3.09668600 & -2.50256300 \\ \mathrm{H} & 4.92621900 & 3.66356600 & -0.35275700 & \mathrm{H} & 3.03106700 & -3.52417300 & -2.67832600 \\ \mathrm{H} & 3.58726500 & 3.33397200 & -1.46270200 & \mathrm{H} & 1.30664300 & -3.85187600 & -2.78318600 \\ \mathrm{H} & 3.32450600 & 3.50413400 & 2.35597700 & \mathrm{H} & 1.91049200 & -2.23666100 & -3.16256700\end{array}$

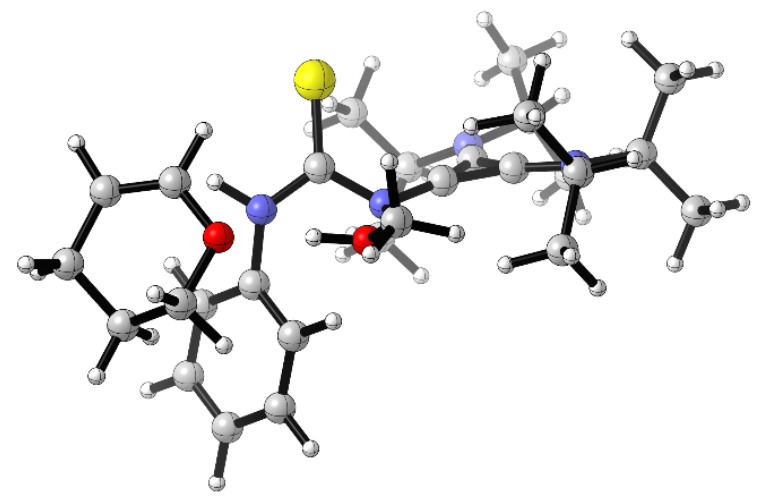

Figure S22. Optimized Structure of $E, Z-1-A n-I_{N} \mathbf{T}_{2}$.

\section{- Thermochemistry -}

\section{(0 imaginary frequencies)}

Zero-point correction $=$

Thermal correction to Energy=

0.728291 (Hartree/Particle)

Thermal correction to Enthalpy=

0.769514

0.770458

Thermal correction to Gibbs Free Energy=

0.651821

Sum of electronic and zero-point Energies $=$

$-1861.892083$

Sum of electronic and thermal Energies=

$-1861.850859$

Sum of electronic and thermal Enthalpies=

$-1861.849915$

Sum of electronic and thermal Free Energies=

$-1861.968552$

$$
\text { C }
$$$$
\begin{array}{lll}
-0.81167100 & -0.47210700 & -1.03059100
\end{array}
$$$$
\begin{array}{lll}
-0.28714800 & -0.66745700 & -2.58947400
\end{array}
$$$$
\begin{array}{llll}
-0.05114600 & 0.20500100 & -0.05259600
\end{array}
$$$$
\begin{array}{lll}
-0.44847700 & 1.12687800 & 0.17979700
\end{array}
$$$$
\begin{array}{lll}
-2.01255100 & -0.89539900 & -0.60429000
\end{array}
$$$$
\begin{array}{lll}
-2.50666000 & -1.13343300 & 0.71042800
\end{array}
$$$$
\begin{array}{lll}
-3.13749900 & -2.35942100 & 0.91823600
\end{array}
$$$$
\begin{array}{lll}
-2.45137200 & -0.19591700 & 1.73676300
\end{array}
$$$$
\begin{array}{lll}
-3.71026900 & -2.64823200 & 2.14797600
\end{array}
$$$$
\begin{array}{lll}
-3.17756900 & -3.08452600 & 0.11252200
\end{array}
$$

$\begin{array}{lrrr}\mathrm{C} & -3.01530600 & -0.50171200 & 2.96953800 \\ \mathrm{H} & -2.01550600 & 0.77989900 & 1.57956400 \\ \mathrm{C} & -3.64710800 & -1.72015600 & 3.18045400 \\ \mathrm{H} & -4.09434700 & -1.94279600 & 4.14156200 \\ \mathrm{H} & -2.58705200 & -1.27434100 & -1.34543700 \\ \mathrm{C} & -4.23132500 & 0.77853200 & -1.88194900 \\ \mathrm{C} & -5.23662600 & -0.07361900 & -2.05485700 \\ \mathrm{C} & -6.28381100 & -0.31052300 & -1.00284800 \\ \mathrm{C} & -5.83236100 & 0.27543100 & 0.33679500 \\ \mathrm{C} & -5.17523700 & 1.62799700 & 0.12314300\end{array}$




\begin{tabular}{|c|c|c|c|c|c|c|c|}
\hline $\mathrm{H}$ & -7.23238900 & 0.13722800 & -1.32010700 & $\mathrm{C}$ & 1.12407100 & -2.99450800 & 1.01972800 \\
\hline $\mathrm{H}$ & -6.47352800 & -1.38244400 & -0.89808100 & $\mathrm{H}$ & 4.88892100 & 0.59234900 & 0.71389600 \\
\hline $\mathrm{H}$ & -6.67681000 & 0.39665300 & 1.01910000 & $\mathrm{C}$ & 5.49094900 & 2.11336200 & -0.66851400 \\
\hline $\mathrm{H}$ & -5.11855100 & -0.39458300 & 0.82103800 & $\mathrm{C}$ & 5.09995400 & 2.38365000 & 1.83015600 \\
\hline $\mathrm{H}$ & -5.88250600 & 2.34141100 & -0.31403700 & $\mathrm{H}$ & 3.40502600 & 3.80123400 & 0.25263500 \\
\hline $\mathrm{H}$ & -4.78148500 & 2.04099400 & 1.05202700 & $\mathrm{C}$ & 1.86126200 & 3.25156100 & -1.10739400 \\
\hline $\mathrm{O}$ & -4.04464100 & 1.53925700 & -0.75995900 & $\mathrm{C}$ & 1.69082500 & 3.31934000 & 1.43238800 \\
\hline $\mathrm{H}$ & -3.44991900 & 0.95165200 & -2.61268400 & $\mathrm{H}$ & 4.71203900 & -1.47340100 & 1.81815400 \\
\hline $\mathrm{H}$ & -5.29984400 & -0.59493000 & -3.00246300 & $\mathrm{H}$ & 4.79544200 & -3.20293300 & 1.45616500 \\
\hline $\mathrm{C}$ & -1.59797100 & 3.84847600 & -0.23923700 & $\mathrm{H}$ & 6.15033500 & -2.15447000 & 1.03820100 \\
\hline $\mathrm{H}$ & -2.51267600 & 4.36380300 & 0.06732400 & $\mathrm{H}$ & 4.70234100 & -3.97378400 & -1.07389200 \\
\hline $\mathrm{H}$ & -1.45754900 & 3.97862600 & -1.31726400 & $\mathrm{H}$ & 4.51222800 & -2.72237100 & -2.31789600 \\
\hline $\mathrm{H}$ & -0.75586000 & 4.30180700 & 0.28224900 & $\mathrm{H}$ & 6.02973000 & -2.87032400 & -1.42168200 \\
\hline $\mathrm{O}$ & -1.64371400 & 2.47582300 & 0.11864100 & $\mathrm{H}$ & 0.89673700 & -2.94772200 & -1.77258500 \\
\hline $\mathrm{H}$ & -2.47662800 & 2.10501100 & -0.22639700 & $\mathrm{H}$ & 2.40934400 & -3.79416200 & -2.12026500 \\
\hline $\mathrm{H}$ & -2.97396400 & 0.23285200 & 3.76514900 & $\mathrm{H}$ & 1.13405400 & -4.61142500 & -1.21014000 \\
\hline $\mathrm{H}$ & -4.20190300 & -3.60174800 & 2.29886800 & $\mathrm{H}$ & 1.53585000 & -2.58801500 & 1.94646200 \\
\hline $\mathrm{C}$ & 1.29250700 & 0.09305800 & -0.06266400 & $\mathrm{H}$ & 0.30238800 & -2.35388500 & 0.69177900 \\
\hline $\mathrm{C}$ & 2.40516800 & -0.71180100 & -0.10293600 & $\mathrm{H}$ & 0.69854300 & -3.97552700 & 1.23807800 \\
\hline $\mathrm{C}$ & 2.52372800 & 0.67241800 & 0.09845500 & $\mathrm{H}$ & 5.32851700 & 3.17539400 & -0.87148200 \\
\hline $\mathrm{N}$ & 2.98011900 & -1.88900300 & -0.22158300 & $\mathrm{H}$ & 6.56004200 & 1.96975600 & -0.49840400 \\
\hline $\mathrm{N}$ & 3.24522600 & 1.75351200 & 0.30509300 & $\mathrm{H}$ & 5.20311200 & 1.54786600 & -1.55815300 \\
\hline $\mathrm{C}$ & 4.45788300 & -1.94766200 & -0.29098200 & $\mathrm{H}$ & 4.52917700 & 2.01870200 & 2.68648700 \\
\hline $\mathrm{C}$ & 2.20019500 & -3.14269800 & -0.05259600 & $\mathrm{H}$ & 6.15974900 & 2.21046100 & 2.02721700 \\
\hline $\mathrm{C}$ & 4.69819500 & 1.65391100 & 0.55148300 & $\mathrm{H}$ & 4.95630800 & 3.46380300 & 1.74965600 \\
\hline $\mathrm{C}$ & 2.58913400 & 3.07733800 & 0.22250500 & $\mathrm{H}$ & 1.48238500 & 4.27236300 & -1.18951200 \\
\hline $\mathrm{C}$ & 5.06049700 & -2.20861500 & 1.08766000 & $\mathrm{H}$ & 2.53494800 & 3.06688800 & -1.94634800 \\
\hline $\mathrm{H}$ & 4.76493400 & -0.95616600 & -0.63167000 & $\mathrm{H}$ & 1.00686800 & 2.57516500 & -1.19826000 \\
\hline $\mathrm{C}$ & 4.94336100 & -2.94188600 & -1.33982400 & $\mathrm{H}$ & 0.82471200 & 2.65082600 & 1.42867600 \\
\hline $\mathrm{C}$ & 1.62931200 & -3.65093000 & -1.37217900 & $\mathrm{H}$ & 2.23792100 & 3.16974600 & 2.36467200 \\
\hline $\mathrm{H}$ & 2.92937700 & -3.86832200 & 0.31570700 & $\mathrm{H}$ & 1.31626300 & 4.34527700 & 1.42069400 \\
\hline
\end{tabular}

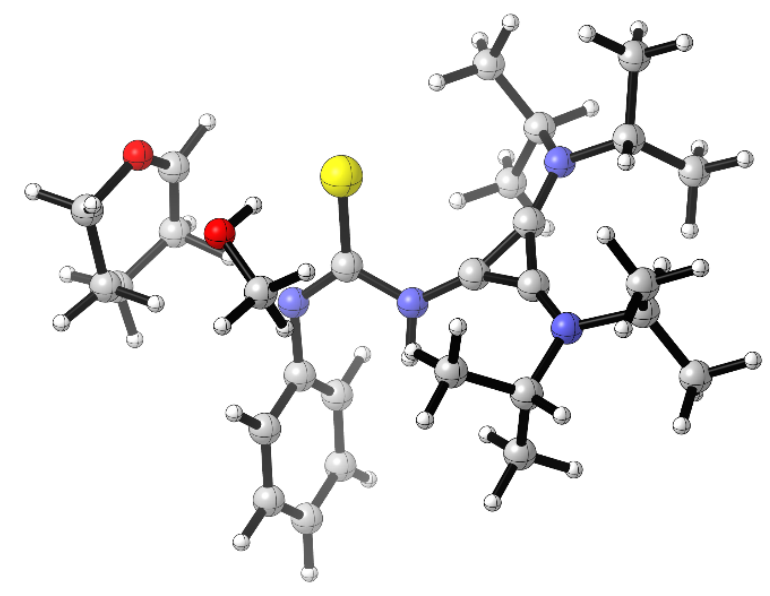

Figure S23. Optimized Structure of E,Z-1-An-TS 1 .

- Thermochemistry -

$(1$ imaginary frequency $=-269.60 \mathrm{~Hz})$

Zero-point correction $=$

Thermal correction to Energy=
0.722611 (Hartree/Particle)

0.763382 


\begin{tabular}{|c|c|c|c|c|c|c|c|}
\hline \multicolumn{5}{|c|}{ Thermal correction to Enthalpy $=\quad 0.764326$} & & & \\
\hline \multicolumn{5}{|c|}{ Thermal correction to Gibbs Free Energy $=0.646270$} & & & \\
\hline \multicolumn{4}{|c|}{ Sum of electronic and zero-point Energies= } & -1861.849215 & & & \\
\hline \multicolumn{4}{|c|}{ Sum of electronic and thermal Energies $=$} & -1861.808444 & & & \\
\hline \multicolumn{4}{|c|}{ Sum of electronic and thermal Enthalpies $=$} & -1861.807500 & & & \\
\hline \multicolumn{4}{|c|}{ Sum of electronic and thermal Free Energies $=$} & -1861.925557 & & & \\
\hline $\mathrm{C}$ & 1.14159700 & -0.37736600 & -0.34932300 & $\mathrm{~N}$ & -3.12524800 & 1.58151600 & 0.75440900 \\
\hline $\mathrm{S}$ & 0.90770400 & -1.89171500 & 0.41135700 & $\mathrm{C}$ & -4.39469300 & -1.96596200 & -0.20246800 \\
\hline $\mathrm{N}$ & 0.01415800 & 0.43592700 & -0.58539300 & $\mathrm{C}$ & -2.44420200 & -2.77910700 & -1.64883400 \\
\hline $\mathrm{H}$ & 0.20588400 & 1.41186600 & -0.77358400 & $\mathrm{C}$ & -4.58644500 & 1.50558700 & 0.95694800 \\
\hline $\mathrm{N}$ & 2.30468400 & 0.09947100 & -0.66834400 & $\mathrm{C}$ & -2.37808200 & 2.77107100 & 1.22375100 \\
\hline $\mathrm{C}$ & 2.46356600 & 1.35597000 & -1.29317400 & $\mathrm{C}$ & -5.34854100 & -1.45087500 & -1.27937100 \\
\hline $\mathrm{C}$ & 2.12747100 & 1.54449600 & -2.63659500 & $\mathrm{H}$ & -4.47955700 & -1.33475600 & 0.68474400 \\
\hline $\mathrm{C}$ & 3.01855600 & 2.41501000 & -0.57128700 & $\mathrm{C}$ & -4.73576600 & -3.38382400 & 0.23966400 \\
\hline $\mathrm{C}$ & 2.33464200 & 2.77784100 & -3.24066300 & $\mathrm{C}$ & -1.72581900 & -3.96746900 & -1.01731800 \\
\hline $\mathrm{H}$ & 1.71288300 & 0.71464400 & -3.19855800 & $\mathrm{H}$ & -3.33291200 & -3.14399700 & -2.17151600 \\
\hline $\mathrm{C}$ & 3.22061400 & 3.64634900 & -1.18160000 & $\mathrm{C}$ & -1.56976000 & -2.06844200 & -2.67681600 \\
\hline $\mathrm{H}$ & 3.29093100 & 2.25766800 & 0.46664200 & $\mathrm{H}$ & -4.90543600 & 0.61035000 & 0.42471400 \\
\hline $\mathrm{C}$ & 2.87920100 & 3.83233100 & -2.51624300 & $\mathrm{C}$ & -4.92180400 & 1.33674200 & 2.43581900 \\
\hline $\mathrm{H}$ & 3.04416600 & 4.79145700 & -2.99209400 & $\mathrm{C}$ & -5.30189800 & 2.69058700 & 0.31485700 \\
\hline $\mathrm{H}$ & 3.46221300 & -0.93373600 & -0.69626800 & $\mathrm{H}$ & -3.10481400 & 3.36040400 & 1.78503400 \\
\hline $\mathrm{C}$ & 4.15789700 & -2.57787200 & 0.19253500 & $\mathrm{C}$ & -1.25822300 & 2.38066000 & 2.18269600 \\
\hline $\mathrm{C}$ & 4.50373100 & -1.57556600 & -0.73782200 & $\mathrm{C}$ & -1.89132200 & 3.61558500 & 0.04901700 \\
\hline $\mathrm{C}$ & 5.57137400 & -0.58400200 & -0.30323000 & $\mathrm{H}$ & -5.09528800 & -0.43124700 & -1.58293900 \\
\hline $\mathrm{C}$ & 5.34565500 & -0.28149200 & 1.17644100 & $\mathrm{H}$ & -5.32106800 & -2.08397200 & -2.16973000 \\
\hline $\mathrm{C}$ & 5.33827400 & -1.55007200 & 1.99954300 & $\mathrm{H}$ & -6.37487400 & -1.45267800 & -0.90509900 \\
\hline $\mathrm{H}$ & 6.57312100 & -0.99061800 & -0.47298700 & $\mathrm{H}$ & -4.70251400 & -4.09444000 & -0.58911300 \\
\hline $\mathrm{H}$ & 5.48531100 & 0.32996200 & -0.89369300 & $\mathrm{H}$ & -4.05959300 & -3.72517300 & 1.02469900 \\
\hline $\mathrm{H}$ & 6.12001900 & 0.37720000 & 1.57555000 & $\mathrm{H}$ & -5.75247200 & -3.39250400 & 0.63784700 \\
\hline $\mathrm{H}$ & 4.37864300 & 0.20829500 & 1.31000300 & $\mathrm{H}$ & -0.83497400 & -3.63007700 & -0.48808200 \\
\hline $\mathrm{H}$ & 6.31811400 & -2.02644300 & 2.06382400 & $\mathrm{H}$ & -2.36595600 & -4.51057200 & -0.32300800 \\
\hline $\mathrm{H}$ & 4.92183700 & -1.38965500 & 2.98961200 & $\mathrm{H}$ & -1.42175300 & -4.66111400 & -1.80542400 \\
\hline $\mathrm{O}$ & 4.45975100 & -2.58547300 & 1.43076400 & $\mathrm{H}$ & -2.07569400 & -1.19856400 & -3.10378000 \\
\hline $\mathrm{H}$ & 3.52170600 & -3.41294900 & -0.08564600 & $\mathrm{H}$ & -0.61744600 & -1.75741300 & -2.24256500 \\
\hline $\mathrm{H}$ & 4.52431200 & -1.94086300 & -1.76364300 & $\mathrm{H}$ & -1.34737900 & -2.76257400 & -3.48917100 \\
\hline $\mathrm{C}$ & 1.91450800 & 0.64969100 & 2.97918600 & $\mathrm{H}$ & -4.61762400 & 2.21063500 & 3.01819600 \\
\hline $\mathrm{H}$ & 0.92514000 & 0.50161500 & 3.43040200 & $\mathrm{H}$ & -5.99920000 & 1.21437400 & 2.56491600 \\
\hline $\mathrm{H}$ & 1.78161500 & 1.15215200 & 2.00947900 & $\mathrm{H}$ & -4.42317000 & 0.45786200 & 2.85135500 \\
\hline $\mathrm{H}$ & 2.49110600 & 1.30893900 & 3.63051700 & $\mathrm{H}$ & -5.05462000 & 2.77174400 & -0.74573500 \\
\hline $\mathrm{O}$ & 2.62621800 & -0.55670900 & 2.85118600 & $\mathrm{H}$ & -6.38126500 & 2.55277900 & 0.40437600 \\
\hline $\mathrm{H}$ & 2.11764700 & -1.13395000 & 2.26260700 & $\mathrm{H}$ & -5.05351500 & 3.63590800 & 0.80366300 \\
\hline $\mathrm{H}$ & 3.65287200 & 4.46223700 & -0.61375700 & $\mathrm{H}$ & -0.80389700 & 3.27945200 & 2.60467500 \\
\hline $\mathrm{H}$ & 2.07879600 & 2.91333600 & -4.28524600 & $\mathrm{H}$ & -1.64333600 & 1.77208100 & 3.00351200 \\
\hline $\mathrm{C}$ & -1.26694200 & 0.12034900 & -0.29042600 & $\mathrm{H}$ & -0.46908200 & 1.81696400 & 1.67997200 \\
\hline $\mathrm{C}$ & -2.36529100 & -0.70747700 & -0.33751800 & $\mathrm{H}$ & -1.17667800 & 3.06809000 & -0.57246100 \\
\hline $\mathrm{C}$ & -2.45576900 & 0.59068800 & 0.20332400 & $\mathrm{H}$ & -2.72300900 & 3.92240200 & -0.58748300 \\
\hline $\mathrm{N}$ & -2.98327200 & -1.82884200 & -0.63678200 & $\mathrm{H}$ & -1.38762400 & 4.51268300 & 0.41416600 \\
\hline
\end{tabular}




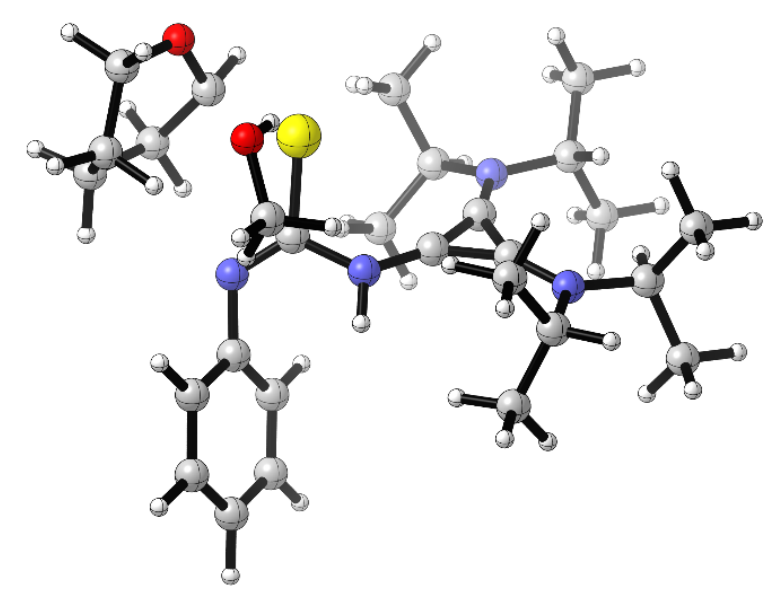

Figure S24. Optimized Structure of $E, Z-\mathbf{1}-A n-\mathbf{I N T}_{3}$.

- Thermochemistry -

(0 imaginary frequencies)

Zero-point correction=

Thermal correction to Energy=

0.726911 (Hartree/Particle)

Thermal correction to Enthalpy=

0.768150

Thermal correction to Gibbs Free Energy=

0.769094

Sum of electronic and zero-point Energies=

0.649656

Sum of electronic and thermal Energies=

$-1861.858585$

Sum of electronic and thermal Enthalpies=

$-1861.817347$

Sum of electronic and thermal Free Energies=

$-1861.816402$

$-1861.935840$

$\begin{array}{lrrr}\mathrm{C} & 1.41940200 & 0.08088000 & -0.11322400 \\ \mathrm{~S} & 1.48062400 & -1.40670100 & 0.82700000 \\ \mathrm{~N} & 0.17641100 & 0.74012600 & 0.04423000 \\ \mathrm{H} & 0.21633100 & 1.74755500 & -0.04588000 \\ \mathrm{~N} & 2.38868200 & 0.59461800 & -0.73680100 \\ \mathrm{C} & 2.23477600 & 1.81158200 & -1.43563100 \\ \mathrm{C} & 1.35985300 & 1.93505500 & -2.51813900 \\ \mathrm{C} & 3.01977700 & 2.89941300 & -1.05436800 \\ \mathrm{C} & 1.26242200 & 3.14321000 & -3.19580900 \\ \mathrm{H} & 0.77918900 & 1.07623600 & -2.83759300 \\ \mathrm{C} & 2.90456100 & 4.10754700 & -1.72926100 \\ \mathrm{H} & 3.71965000 & 2.78189000 & -0.23485100 \\ \mathrm{C} & 2.02593100 & 4.23500000 & -2.79887300 \\ \mathrm{H} & 1.94773900 & 5.17598200 & -3.32967800 \\ \mathrm{H} & 3.15111600 & -1.61072100 & -1.62674000 \\ \mathrm{C} & 3.01749500 & -2.50524400 & 0.32884600 \\ \mathrm{C} & 3.81927100 & -2.04035100 & -0.88072400 \\ \mathrm{C} & 4.97074000 & -1.07767300 & -0.52609100 \\ \mathrm{C} & 4.90517200 & -0.64464100 & 0.93533000 \\ \mathrm{C} & 4.77551100 & -1.84299000 & 1.85414600 \\ \mathrm{H} & 5.92652100 & -1.57700400 & -0.70824800 \\ \mathrm{H} & 4.93016600 & -0.20112200 & -1.17243800 \\ \mathrm{H} & 5.80844100 & -0.09872100 & 1.21794500 \\ \mathrm{H} & 4.06674800 & 0.03590700 & 1.09107200 \\ \mathrm{H} & 5.69780100 & -2.43021300 & 1.86147800\end{array}$

$\begin{array}{lrrr}\mathrm{H} & 4.54610900 & -1.53037100 & 2.87335400 \\ \mathrm{O} & 3.73167500 & -2.76370000 & 1.45565800 \\ \mathrm{H} & 2.47185300 & -3.41979500 & 0.09897400 \\ \mathrm{H} & 4.22646800 & -2.96072600 & -1.30996700 \\ \mathrm{C} & 2.37918100 & 1.61521200 & 2.96316900 \\ \mathrm{H} & 1.30894500 & 1.77422900 & 3.15555300 \\ \mathrm{H} & 2.57260800 & 1.77232500 & 1.89178800 \\ \mathrm{H} & 2.93533100 & 2.37049900 & 3.51910000 \\ \mathrm{O} & 2.81942200 & 0.35428100 & 3.40773200 \\ \mathrm{H} & 2.33840700 & -0.31839000 & 2.91787900 \\ \mathrm{H} & 3.51500600 & 4.94957000 & -1.42472200 \\ \mathrm{H} & 0.59197600 & 3.22891300 & -4.04328400 \\ \mathrm{C} & -1.04931800 & 0.18250700 & 0.06913200 \\ \mathrm{C} & -1.96106800 & -0.80204900 & -0.24168100 \\ \mathrm{C} & -2.36914300 & 0.38519000 & 0.38908800 \\ \mathrm{~N} & -2.30285900 & -1.97123700 & -0.74571200 \\ \mathrm{~N} & -3.28525500 & 1.18484700 & 0.89010600 \\ \mathrm{C} & -3.71470900 & -2.39857000 & -0.58273400 \\ \mathrm{C} & -1.44503400 & -2.68479400 & -1.72862900 \\ \mathrm{C} & -4.72457700 & 0.85808600 & 0.79985200 \\ \mathrm{C} & -2.85327500 & 2.43938100 & 1.55043000 \\ \mathrm{C} & -4.56204400 & -1.93778700 & -1.76768400 \\ \mathrm{H} & -4.05901000 & -1.89550100 & 0.32342300 \\ \mathrm{C} & -3.84308100 & -3.89512500 & -0.32562000 \\ \mathrm{C} & -0.59571900 & -3.76995000 & -1.07420900\end{array}$




$\begin{array}{lrrrrrrr}\mathrm{H} & -2.15399000 & -3.16173600 & -2.41008100 & \mathrm{H} & -0.00659000 & -4.28298800 & -1.83843400 \\ \mathrm{C} & -0.60566400 & -1.72048500 & -2.56125300 & \mathrm{H} & -1.21562400 & -0.91279700 & -2.97402400 \\ \mathrm{H} & -4.77915100 & -0.03365700 & 0.17625300 & \mathrm{H} & 0.21516400 & -1.29077800 & -1.98356700 \\ \mathrm{C} & -5.29471200 & 0.52359700 & 2.17445500 & \mathrm{H} & -0.15982100 & -2.26491200 & -3.39535400 \\ \mathrm{C} & -5.49983700 & 1.95973200 & 0.08292100 & \mathrm{H} & -5.25773600 & 1.38445100 & 2.84725000 \\ \mathrm{H} & -3.75685300 & 2.84501300 & 2.00756600 & \mathrm{H} & -6.34120300 & 0.22635900 & 2.08045300 \\ \mathrm{C} & -1.85180900 & 2.16324100 & 2.66746400 & \mathrm{H} & -4.74338800 & -0.29765500 & 2.63826800 \\ \mathrm{C} & -2.34475100 & 3.45307000 & 0.52822400 & \mathrm{H} & -5.07480600 & 2.15921300 & -0.90312900 \\ \mathrm{H} & -4.45598800 & -0.86290100 & -1.93884800 & \mathrm{H} & -6.53722300 & 1.64653600 & -0.04973800 \\ \mathrm{H} & -4.27629100 & -2.45549500 & -2.68675700 & \mathrm{H} & -5.51169000 & 2.89230900 & 0.65241600 \\ \mathrm{H} & -5.61687300 & -2.15333900 & -1.58360800 & \mathrm{H} & -1.63865500 & 3.08807800 & 3.20690400 \\ \mathrm{H} & -3.53665200 & -4.49051800 & -1.18857500 & \mathrm{H} & -2.24851500 & 1.43543300 & 3.37812300 \\ \mathrm{H} & -3.25514800 & -4.19972000 & 0.54159000 & \mathrm{H} & -0.90366300 & 1.78248800 & 2.27873200 \\ \mathrm{H} & -4.89077300 & -4.12769300 & -0.12540300 & \mathrm{H} & -1.45830700 & 3.08789300 & 0.00100500 \\ \mathrm{H} & 0.08364700 & -3.32554200 & -0.34683200 & \mathrm{H} & -3.10715000 & 3.67665800 & -0.21957700 \\ \mathrm{H} & -1.20811700 & -4.51365900 & -0.56553600 & \mathrm{H} & -2.06971900 & 4.38424800 & 1.02707600\end{array}$

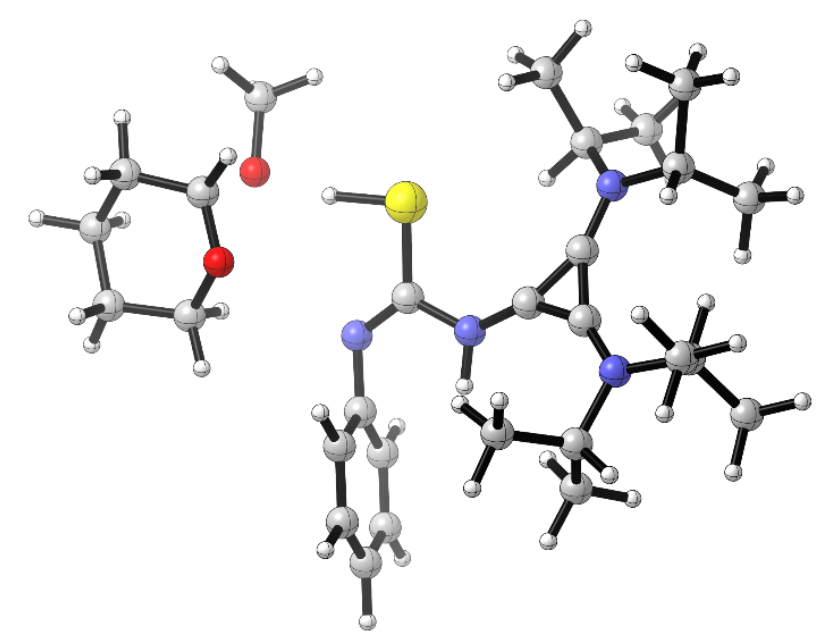

Figure S25. Optimized Structure of E,Z-1-An-TS 2 .

- Thermochemistry -

$(1$ imaginary frequencies $=-283.97 \mathrm{~Hz})$

Zero-point correction $=$

Thermal correction to Energy=

Thermal correction to Enthalpy=

Thermal correction to Gibbs Free Energy=

Sum of electronic and zero-point Energies=

Sum of electronic and thermal Energies=

Sum of electronic and thermal Enthalpies=

Sum of electronic and thermal Free Energies=
0.723228 (Hartree/Particle)

$$
0.762111
$$

0.763056

0.650594

$-1861.852330$

$-1861.813447$

$-1861.812502$

$-1861.924964$ 


$\begin{array}{lrrr}\mathrm{C} & 1.06625800 & -0.00601700 & -0.73137300 \\ \mathrm{~S} & 1.12031800 & -1.72901200 & -0.43440800 \\ \mathrm{~N} & 2.09025700 & 0.68952800 & -1.00298800 \\ \mathrm{~N} & -0.20155500 & 0.60706500 & -0.61768700 \\ \mathrm{C} & 1.96502900 & 2.08966700 & -1.10177800 \\ \mathrm{C} & 1.88168900 & 2.71058100 & -2.34887600 \\ \mathrm{C} & 1.94300400 & 2.87068100 & 0.05898200 \\ \mathrm{C} & 1.76268000 & 4.09214400 & -2.42889200 \\ \mathrm{H} & 1.91515000 & 2.10074700 & -3.24414100 \\ \mathrm{C} & 1.82571800 & 4.25262500 & -0.03230300 \\ \mathrm{H} & 2.03013300 & 2.37977100 & 1.02298800 \\ \mathrm{C} & 1.73053800 & 4.86789600 & -1.27478800 \\ \mathrm{H} & 1.64487800 & 5.94548000 & -1.34593200 \\ \mathrm{H} & 6.08517700 & -2.92092000 & 1.43492300 \\ \mathrm{C} & 4.42571600 & -1.56589700 & 1.41921000 \\ \mathrm{C} & 5.89432100 & -1.85791500 & 1.58637700 \\ \mathrm{C} & 6.75427400 & -0.98458700 & 0.67847300 \\ \mathrm{C} & 6.31811800 & 0.47102800 & 0.83000700 \\ \mathrm{C} & 4.83519900 & 0.59261400 & 0.54130000 \\ \mathrm{H} & 7.80930700 & -1.10897300 & 0.92926900 \\ \mathrm{H} & 6.61952400 & -1.30323900 & -0.35892800 \\ \mathrm{H} & 6.52901300 & 0.82346700 & 1.84552800 \\ \mathrm{H} & 6.86253300 & 1.11993500 & 0.13965500 \\ \mathrm{H} & 4.45056000 & 1.58656600 & 0.76205300 \\ \mathrm{H} & 4.60194900 & 0.35332400 & -0.49741700 \\ \mathrm{O} & 4.04495200 & -0.29238100 & 1.37159000 \\ \mathrm{H} & 6.12089300 & -1.64701100 & 2.63927200 \\ \mathrm{C} & 4.00028400 & -3.62197100 & -0.21131600 \\ \mathrm{H} & 2.97412900 & -4.01813100 & -0.19525600 \\ \mathrm{H} & 4.53665900 & -4.07423500 & 0.62934100 \\ \mathrm{H} & 4.48359000 & -3.94472600 & -1.13669900 \\ \mathrm{O} & 3.99844100 & -2.21306700 & -0.14962000 \\ \mathrm{H} & 2.56541700 & -1.82019200 & -0.35769000 \\ \mathrm{C} & -1.39187400 & 0.11316900 & -0.23652500 \\ \mathrm{C} & -2.38116600 & -0.83992300 & -0.12408400 \\ \mathrm{C} & -2.61246100 & 0.48315800 & 0.27522100 \\ \mathrm{~N} & -2.83684100 & -2.07040300 & -0.26579800 \\ \mathrm{~N} & -3.36825200 & 1.45456600 & 0.74613200 \\ \mathrm{C} & -4.19654600 & -2.33458100 & 0.26944700 \\ \mathrm{C} & -2.06143100 & -3.00331100 & -1.12744800 \\ \mathrm{C} & -4.79373400 & 1.23485300 & 1.06528700\end{array}$

$\begin{array}{lrrr}\mathrm{C} & -2.77196300 & 2.79866900 & 0.92930100 \\ \mathrm{C} & -5.29346600 & -1.95161800 & -0.72617400 \\ \mathrm{H} & -4.27330300 & -1.67373400 & 1.13754700 \\ \mathrm{C} & -4.37496400 & -3.75017400 & 0.80277000 \\ \mathrm{C} & -2.85478000 & -3.47236500 & -2.34374500 \\ \mathrm{H} & -4.99041000 & 0.19483600 & 0.80871100 \\ \mathrm{C} & -5.05612900 & 1.41371900 & 2.55771600 \\ \mathrm{C} & -5.69395400 & 2.10525300 & 0.19336500 \\ \mathrm{H} & -3.55509900 & 3.39028600 & 1.40553800 \\ \mathrm{C} & -1.58375600 & 2.75841300 & 1.88627500 \\ \mathrm{C} & -2.44838600 & 3.45479600 & -0.41155900 \\ \mathrm{H} & -5.11482100 & -0.96331400 & -1.15882600 \\ \mathrm{H} & -5.36341700 & -2.66934400 & -1.54396800 \\ \mathrm{H} & -6.26108900 & -1.93039200 & -0.21822100 \\ \mathrm{H} & -4.37129100 & -4.49726900 & 0.00792700 \\ \mathrm{H} & -3.60360100 & -4.00322000 & 1.53077700 \\ \mathrm{H} & -5.34503400 & -3.81030700 & 1.30046100 \\ \mathrm{H} & -3.66413100 & -4.15374000 & -2.07410100 \\ \mathrm{H} & -3.27289700 & -2.62932000 & -2.89734400 \\ \mathrm{H} & -2.18241200 & -4.01559100 & -3.01096100 \\ \mathrm{H} & -4.88486200 & 2.44423900 & 2.87956200 \\ \mathrm{H} & -6.09717300 & 1.17171500 & 2.78128300 \\ \mathrm{H} & -4.41452200 & 0.75716600 & 3.14956400 \\ \mathrm{H} & -5.55290500 & 3.16957200 & 0.39883600 \\ \mathrm{H} & -5.50012200 & 1.92901600 & -0.86704000 \\ \mathrm{H} & -6.74108000 & 1.86969300 & 0.39389700 \\ \mathrm{H} & -1.24183600 & 3.77534400 & 2.08861100 \\ \mathrm{H} & -1.86469000 & 2.29361200 & 2.83343800 \\ \mathrm{H} & -0.73472900 & 2.20886700 & 1.47228300 \\ \mathrm{H} & -3.34599700 & 3.55183100 & -1.02501700 \\ \mathrm{H} & -2.02906800 & 4.44973600 & -0.25091000 \\ \mathrm{H} & -1.71484400 & 2.88462600 & -0.98864000 \\ \mathrm{H} & 1.69901400 & 4.56638900 & -3.40146200 \\ \mathrm{H} & 1.81909600 & 4.84949100 & 0.87297400 \\ \mathrm{H} & -1.23772900 & -2.39167700 & -1.49697100 \\ \mathrm{C} & -1.43426600 & -4.16005500 & -0.35259800 \\ \mathrm{H} & -2.15250100 & -4.94475200 & -0.11475200 \\ \mathrm{H} & -0.64911300 & -4.60491500 & -0.96874000 \\ & -0.97487700 & -3.80543300 & 0.57132700 \\ & 3.75494300 & -2.12629900 & 2.06876500 \\ -0.14961300 & 1.61839500 & -0.64799900\end{array}$




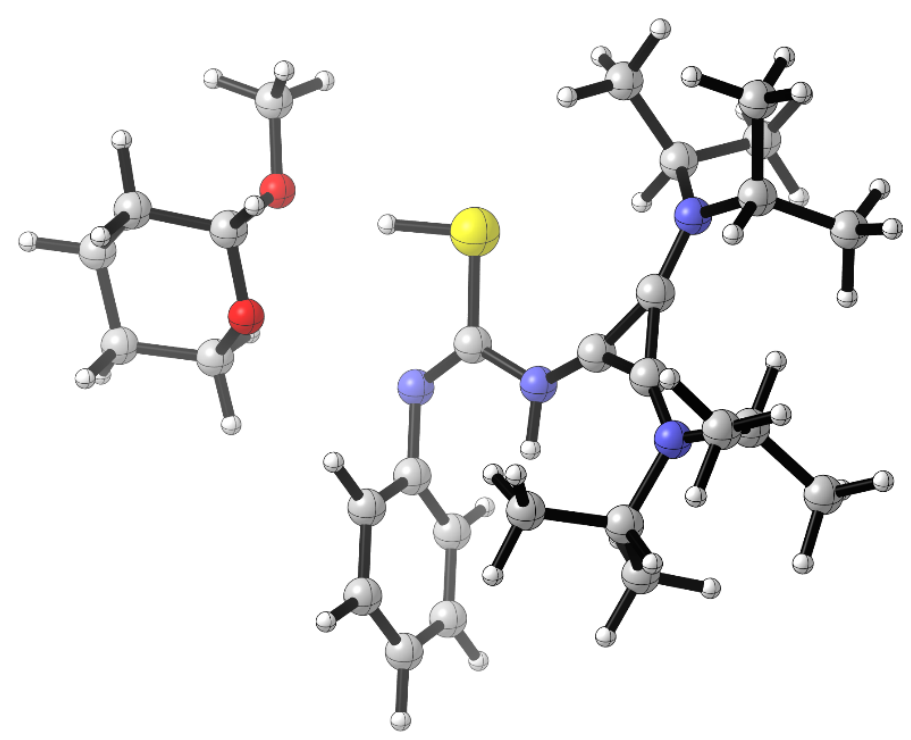

Figure S26. Optimized Structure of $E, Z-1-A n-I_{N} \mathbf{T}_{4}$.

- Thermochemistry -

(0 imaginary frequencies)

Zero-point correction=

Thermal correction to Energy=

Thermal correction to Enthalpy=

Thermal correction to Gibbs Free Energy=

Sum of electronic and zero-point Energies=

Sum of electronic and thermal Energies=

Sum of electronic and thermal Enthalpies=

Sum of electronic and thermal Free Energies=
0.726802 (Hartree/Particle)

0.766493

0.767438

0.652288

$-1861.872595$

$-1861.832904$

$-1861.831960$

$-1861.947109$

$\begin{array}{lrrr}\mathrm{C} & -1.01664000 & -0.04141400 & 1.07628000 \\ \mathrm{~S} & -1.04115500 & -1.79748600 & 0.94923800 \\ \mathrm{~N} & -2.05557800 & 0.63777100 & 1.30669300 \\ \mathrm{~N} & 0.23268200 & 0.58292200 & 0.87851900 \\ \mathrm{C} & -1.97947500 & 2.04418900 & 1.21181000 \\ \mathrm{C} & -1.74291900 & 2.82935300 & 2.34052300 \\ \mathrm{C} & -2.15065300 & 2.65187000 & -0.03575200 \\ \mathrm{C} & -1.66331300 & 4.21174000 & 2.21488500 \\ \mathrm{H} & -1.63154000 & 2.34991700 & 3.30623700 \\ \mathrm{C} & -2.07069400 & 4.03382900 & -0.14766300 \\ \mathrm{H} & -2.36232500 & 2.02419800 & -0.89529900 \\ \mathrm{C} & -1.82137100 & 4.81781800 & 0.97403000 \\ \mathrm{H} & -1.76656200 & 5.89605800 & 0.88364700 \\ \mathrm{H} & -5.75084800 & -2.79031600 & -2.16309900 \\ \mathrm{C} & -4.19444000 & -1.51566100 & -1.36159900 \\ \mathrm{C} & -5.59104800 & -1.73380100 & -1.93545500 \\ \mathrm{C} & -6.65552800 & -1.18694000 & -0.98664000 \\ \mathrm{C} & -6.32388100 & 0.26700500 & -0.65034500\end{array}$

$\begin{array}{lrrr}\mathrm{C} & -4.90095800 & 0.37622100 & -0.12665300 \\ \mathrm{H} & -7.64490200 & -1.26678900 & -1.44215200 \\ \mathrm{H} & -6.67234000 & -1.78212000 & -0.06734900 \\ \mathrm{H} & -6.42718200 & 0.88459300 & -1.54936600 \\ \mathrm{H} & -7.01226700 & 0.66416500 & 0.10050900 \\ \mathrm{H} & -4.59697300 & 1.41564700 & -0.01101200 \\ \mathrm{H} & -4.79497100 & -0.11765500 & 0.84319000 \\ \mathrm{O} & -3.95148300 & -0.19202000 & -1.04252700 \\ \mathrm{H} & -5.62650000 & -1.19690200 & -2.88863100 \\ \mathrm{C} & -4.07208400 & -3.68137700 & -0.30588400 \\ \mathrm{H} & -3.50311200 & -4.02833300 & -1.17856100 \\ \mathrm{H} & -5.11048900 & -4.01222700 & -0.40144300 \\ \mathrm{H} & -3.64967600 & -4.12643100 & 0.59591000 \\ \mathrm{O} & -3.98287000 & -2.27963800 & -0.15495500 \\ \mathrm{H} & -2.38829400 & -1.85796400 & 0.59679300 \\ \mathrm{C} & 1.39390000 & 0.11757200 & 0.38132800 \\ \mathrm{C} & 2.39905100 & -0.80351500 & 0.17884100 \\ \mathrm{C} & 2.54906500 & 0.52561700 & -0.24175000\end{array}$




$\begin{array}{rrrrrrrr}\mathrm{N} & 2.90666000 & -2.01731200 & 0.27703700 & \mathrm{H} & 3.62741600 & -2.63529900 & 2.83420300 \\ \mathrm{~N} & 3.22865800 & 1.51482000 & -0.78304300 & \mathrm{H} & 2.56663700 & -4.03597200 & 3.02672800 \\ \mathrm{C} & 4.21306600 & -2.24516800 & -0.39263600 & \mathrm{H} & 4.56675000 & 2.52611600 & -3.03135900 \\ \mathrm{C} & 2.23919500 & -2.98043800 & 1.19336500 & \mathrm{H} & 5.81724800 & 1.28794700 & -3.00171500 \\ \mathrm{C} & 4.63539400 & 1.33993700 & -1.20073200 & \mathrm{H} & 4.12565600 & 0.82328100 & -3.24778400 \\ \mathrm{C} & 2.57367100 & 2.83616000 & -0.93621700 & \mathrm{H} & 5.37924300 & 3.30800500 & -0.61922300 \\ \mathrm{C} & 5.39322000 & -1.86139200 & 0.50215300 & \mathrm{H} & 5.43838700 & 2.09224000 & 0.67035800 \\ \mathrm{H} & 4.19459000 & -1.56543100 & -1.24906000 & \mathrm{H} & 6.60143800 & 2.04052100 & -0.66293900 \\ \mathrm{C} & 4.36397300 & -3.64593500 & -0.97203200 & \mathrm{H} & 0.91559200 & 3.73765700 & -1.97486600 \\ \mathrm{C} & 3.16096300 & -3.46959800 & 2.30685700 & \mathrm{H} & 1.53455900 & 2.27155600 & -2.75415300 \\ \mathrm{H} & 4.88573700 & 0.31125200 & -0.94508000 & \mathrm{H} & 0.52195400 & 2.16449000 & -1.30386300 \\ \mathrm{C} & 4.78775600 & 1.50518300 & -2.70961300 & \mathrm{H} & 3.27532300 & 3.64401100 & 0.95463000 \\ \mathrm{C} & 5.56237000 & 2.25230000 & -0.40295200 & \mathrm{H} & 1.87131100 & 4.48242400 & 0.27284200 \\ \mathrm{H} & 3.29526300 & 3.44440900 & -1.48343900 & \mathrm{H} & 1.66986100 & 2.92247700 & 1.05816400 \\ \mathrm{C} & 1.31416500 & 2.73797600 & -1.79184800 & \mathrm{H} & -1.48355000 & 4.81696400 & 3.09594400 \\ \mathrm{C} & 2.33619000 & 3.50528800 & 0.41606300 & \mathrm{H} & -2.21406200 & 4.50040400 & -1.11577100 \\ \mathrm{H} & 5.24240100 & -0.88237400 & 0.96542300 & \mathrm{H} & 1.45685000 & -2.38805700 & 1.66971000 \\ \mathrm{H} & 5.54810300 & -2.59056700 & 1.29786100 & \mathrm{C} & 1.54747200 & -4.12548600 & 0.45689300 \\ \mathrm{H} & 6.30887500 & -1.81881500 & -0.09320800 & \mathrm{H} & 2.24969900 & -4.88461900 & 0.11261600 \\ \mathrm{H} & 4.44881900 & -4.40908400 & -0.19742000 & \mathrm{H} & 0.84668100 & -4.60943900 & 1.14149600 \\ \mathrm{H} & 3.53200600 & -3.89901900 & -1.63015500 & \mathrm{H} & 0.98052500 & -3.75152900 & -0.39705700 \\ \mathrm{H} & 5.28366600 & -3.67654500 & -1.55991900 & \mathrm{H} & -3.42097500 & -1.80373300 & -2.08468600 \\ \mathrm{H} & 3.94301600 & -4.13526000 & 1.93680900 & \mathrm{H} & 0.15661700 & 1.59378800 & 0.89160600\end{array}$

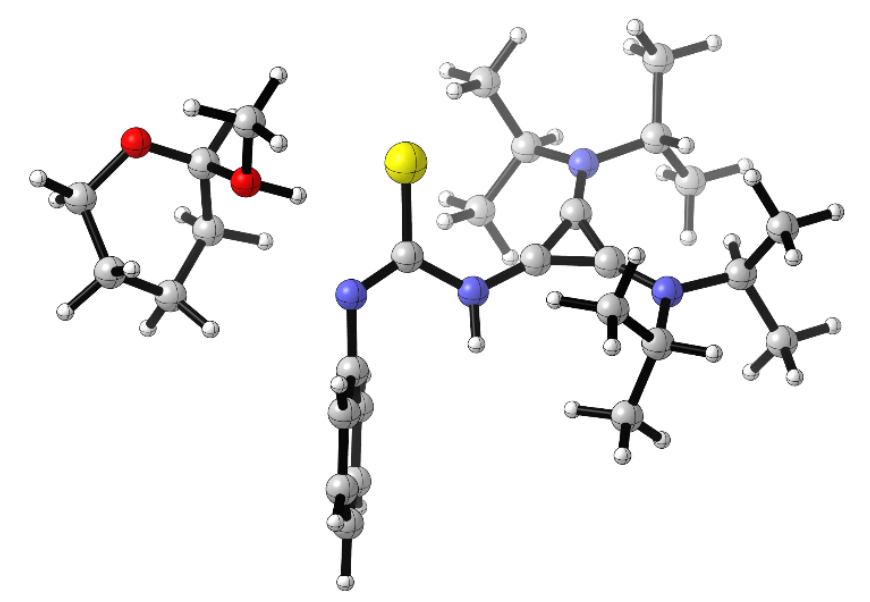

Figure S27. Optimized Structure of E,Z-1-An-TS3-PA.

- Thermochemistry -

$(1$ imaginary frequency $=-273.08 \mathrm{~Hz})$

Zero-point correction $=$

Thermal correction to Energy=

Thermal correction to Enthalpy=

Thermal correction to Gibbs Free Energy=

Sum of electronic and zero-point Energies $=$

Sum of electronic and thermal Energies=

Sum of electronic and thermal Enthalpies=
0.727902 (Hartree/Particle)

0.767538

0.768482

0.652541

$-1861.856001$

$-1861.816366$

$-1861.815422$ 
$\mathrm{N}$

C

C

$\mathrm{C}$

C

$\mathrm{H}$

C

$\mathrm{H}$

C

$\mathrm{H}$

C

$\begin{array}{rrr}3.78210700 & 1.23690200 & -0.65479400 \\ 4.20963600 & -2.47193400 & 0.43223000 \\ 1.92300100 & -2.90614700 & 1.49466100 \\ 5.21916500 & 0.90573400 & -0.58358400 \\ 3.34983100 & 2.52932800 & -1.23473000 \\ 5.02910100 & -2.13119500 & 1.67665800 \\ 4.56518700 & -1.86988900 & -0.40688600 \\ 4.36645100 & -3.93038600 & 0.01929900 \\ 1.11496600 & -3.94044000 & 0.71737300 \\ 2.62492900 & -3.42876900 & 2.15059000 \\ 1.03193300 & -2.03915500 & 2.37873300 \\ 5.27406500 & -0.01983800 & -0.01145800 \\ 5.79242800 & 0.65078500 & -1.97452100 \\ 5.99655900 & 1.96400600 & 0.19426000 \\ 4.25527800 & 2.96726600 & -1.65796500 \\ 2.35808400 & 2.32458500 & -2.37665400 \\ 2.82825000 & 3.47450500 & -0.15518500 \\ 4.90404100 & -1.08232400 & 1.95957100 \\ 4.73151800 & -2.74758900 & 2.52864400 \\ 6.09028200 & -2.31366200 & 1.49242300 \\ 4.04735100 & -4.61844200 & 0.80528600 \\ 3.80217200 & -4.14605400 & -0.88920200 \\ 5.42195100 & -4.12760200 & -0.17914100 \\ 0.40340100 & -3.43949600 & 0.06076800 \\ 1.75276500 & -4.59011400 & 0.11860000 \\ 0.56056400 & -4.56689700 & 1.42100100 \\ 1.60855400 & -1.27175900 & 2.90175000 \\ 0.23776400 & -1.56399400 & 1.79910800 \\ 0.55592900 & -2.67353800 & 3.12877900 \\ 5.75519700 & 1.55033400 & -2.59471300 \\ 6.83875200 & 0.34737200 & -1.89799500 \\ 5.23958900 & -0.14073400 & -2.48612200 \\ 5.56703000 & 2.11337400 & 1.18714700 \\ 7.03203500 & 1.63948600 & 0.31501800 \\ 6.01476400 & 2.92533700 & -0.32523100 \\ 2.15870000 & 3.28111300 & -2.86375200 \\ 2.76031400 & 1.63596500 & -3.12248500 \\ 1.40280400 & 1.92706900 & -2.02450800 \\ 1.93407600 & 3.07578800 & 0.33241900 \\ 3.58152000 & 3.64730100 & 0.61524900 \\ 2.55918400 & 4.43632400 & -0.59605300\end{array}$




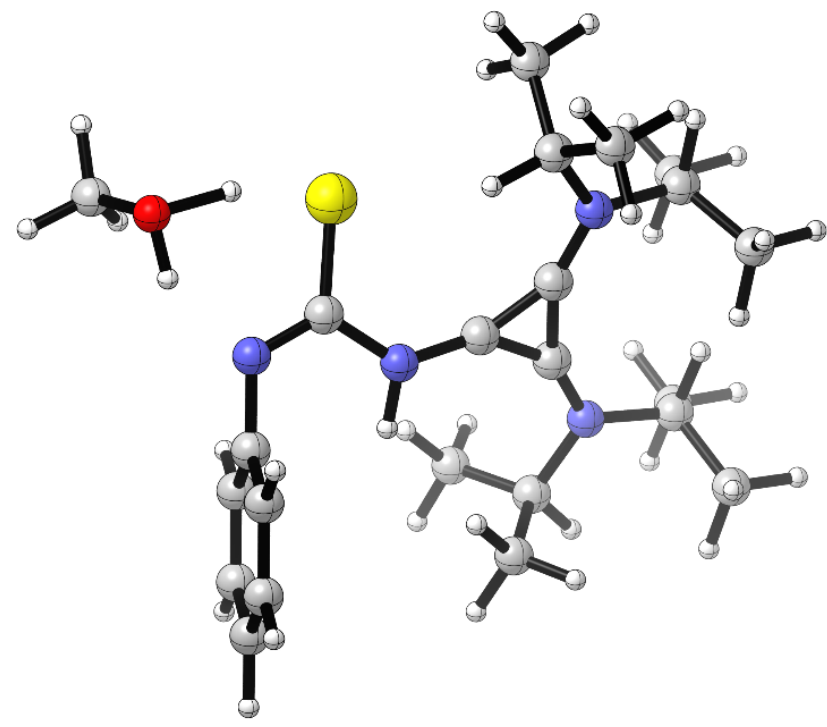

Figure S28. Optimized Structure of E,Z-1-An-TS 3 -MA.

- Thermochemistry -

$(1$ imaginary frequency $=-725.18 \mathrm{~Hz})$

Zero-point correction=

Thermal correction to Energy=

Thermal correction to Enthalpy=

Thermal correction to Gibbs Free Energy=

Sum of electronic and zero-point Energies=

Sum of electronic and thermal Energies=

Sum of electronic and thermal Enthalpies=

Sum of electronic and thermal Free Energies=
0.596299 (Hartree/Particle)

$$
0.630391
$$

0.631335

0.528663

$-1591.443262$

$-1591.409169$

$-1591.408225$

$-1591.510897$

$\begin{array}{lrrr}\mathrm{C} & 1.92032800 & 1.02343500 & 0.08929100 \\ \mathrm{~S} & 1.35153500 & 2.57634200 & 0.63804100 \\ \mathrm{~N} & 0.96890900 & 0.03010300 & -0.16349700 \\ \mathrm{H} & 1.36931700 & -0.89259600 & -0.28524400 \\ \mathrm{~N} & 3.16967000 & 0.74532100 & -0.05046300 \\ \mathrm{C} & 3.54614200 & -0.56839100 & -0.43426000 \\ \mathrm{C} & 3.67718000 & -1.56477000 & 0.53548300 \\ \mathrm{C} & 3.78735500 & -0.87162000 & -1.77378400 \\ \mathrm{C} & 4.03445500 & -2.85545900 & 0.16284100 \\ \mathrm{H} & 3.50837200 & -1.31545200 & 1.57770600 \\ \mathrm{C} & 4.14653300 & -2.16361200 & -2.13675400 \\ \mathrm{H} & 3.69477200 & -0.09000700 & -2.51893500 \\ \mathrm{C} & 4.26723800 & -3.15884500 & -1.17313000 \\ \mathrm{H} & 4.55387300 & -4.16316400 & -1.46104300 \\ \mathrm{H} & 4.33785000 & -2.39111100 & -3.17892400 \\ \mathrm{H} & 4.14341000 & -3.62129800 & 0.92223200 \\ \mathrm{C} & -0.37744700 & 0.06207000 & -0.06378800 \\ \mathrm{C} & -1.62615100 & 0.63333400 & -0.17206100 \\ \mathrm{C} & -1.47410000 & -0.73541200 & 0.13217900 \\ \mathrm{~N} & -2.38710700 & 1.67965900 & -0.40106100 \\ \mathrm{~N} & -1.91342100 & -1.95060600 & 0.39295200\end{array}$

$\begin{array}{lrrr}\mathrm{C} & -1.78004300 & 2.94173900 & -0.89224200 \\ \mathrm{C} & -3.85238800 & 1.61232400 & -0.21164500 \\ \mathrm{C} & -3.35324100 & -2.27709400 & 0.44627400 \\ \mathrm{C} & -0.91933800 & -3.02584100 & 0.62770600 \\ \mathrm{C} & -2.33510100 & 3.31970000 & -2.26191900 \\ \mathrm{H} & -0.71652100 & 2.72937900 & -0.99862400 \\ \mathrm{C} & -1.93404400 & 4.05273100 & 0.13949600 \\ \mathrm{C} & -4.20117700 & 1.23284800 & 1.22535700 \\ \mathrm{H} & -4.19709500 & 2.63687600 & -0.35554800 \\ \mathrm{C} & -4.51281500 & 0.76100700 & -1.29584200 \\ \mathrm{H} & -3.86912100 & -1.33930800 & 0.25415600 \\ \mathrm{C} & -3.73717200 & -3.25891600 & -0.65616900 \\ \mathrm{C} & -3.75967800 & -2.75171000 & 1.83877100 \\ \mathrm{H} & -1.51394300 & -3.90408600 & 0.88171500 \\ \mathrm{C} & -0.13952300 & -3.35826000 & -0.64293600 \\ \mathrm{C} & -0.03032000 & -2.71440600 & 1.82869400 \\ \mathrm{H} & -2.20008700 & 2.50909500 & -2.98138700 \\ \mathrm{H} & -3.39713400 & 3.57668600 & -2.22012400 \\ \mathrm{H} & -1.80297200 & 4.19684100 & -2.63498800 \\ \mathrm{H} & -2.98077000 & 4.33241300 & 0.28822400 \\ \mathrm{H} & -1.50927100 & 3.75048100 & 1.09825400\end{array}$




$\begin{array}{rrrrrrrr}\mathrm{H} & -1.40172300 & 4.94266300 & -0.20267200 & \mathrm{H} & 0.55732700 & -4.17597000 & -0.44983900 \\ \mathrm{H} & -3.79524700 & 0.26154600 & 1.51477400 & \mathrm{H} & -0.81534800 & -3.66185200 & -1.44446900 \\ \mathrm{H} & -3.80283700 & 1.97739700 & 1.91748700 & \mathrm{H} & 0.44917100 & -2.51376500 & -1.01184900 \\ \mathrm{H} & -5.28487700 & 1.19273800 & 1.35128700 & \mathrm{H} & 0.62236800 & -1.85617100 & 1.65202400 \\ \mathrm{H} & -4.38426300 & 1.22689300 & -2.27424100 & \mathrm{H} & -0.63480100 & -2.50699700 & 2.71391100 \\ \mathrm{H} & -4.08437700 & -0.24142500 & -1.35502400 & \mathrm{H} & 0.61017600 & -3.57210000 & 2.04373200 \\ \mathrm{H} & -5.58312600 & 0.66201900 & -1.10356300 & \mathrm{H} & 2.84630000 & 3.10573400 & 0.67627000 \\ \mathrm{H} & -3.25451700 & -4.23050600 & -0.52175100 & \mathrm{O} & 4.11089100 & 3.12183200 & 0.60760100 \\ \mathrm{H} & -4.81631600 & -3.42466300 & -0.64182400 & \mathrm{H} & 4.13678800 & 2.18974700 & 0.25582400 \\ \mathrm{H} & -3.46317400 & -2.86966800 & -1.63952900 & \mathrm{C} & 4.70982200 & 3.14506500 & 1.91212000 \\ \mathrm{H} & -3.48026900 & -2.01882600 & 2.59905900 & \mathrm{H} & 5.77710200 & 2.94888700 & 1.81513400 \\ \mathrm{H} & -4.84214100 & -2.88927800 & 1.87738300 & \mathrm{H} & 4.55965800 & 4.14137500 & 2.32246800 \\ \mathrm{H} & -3.30009300 & -3.70892600 & 2.09727600 & \mathrm{H} & 4.24640400 & 2.39943900 & 2.56357800\end{array}$

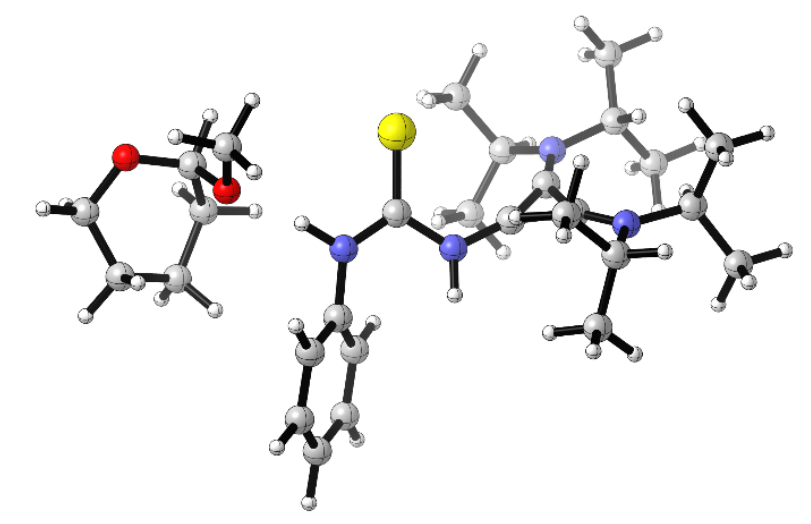

Figure S29. Optimized Structure of E,Z-1-An-INT 5.

- Thermochemistry -

(0 imaginary frequencies)

Zero-point correction=

Thermal correction to Energy=

Thermal correction to Enthalpy=

Thermal correction to Gibbs Free Energy=

Sum of electronic and zero-point Energies=

Sum of electronic and thermal Energies=

Sum of electronic and thermal Enthalpies=

Sum of electronic and thermal Free Energies=
0.729562 (Hartree/Particle)

0.769857

0.770801

0.650830

$-1861.900872$

$-1861.860577$

$-1861.859633$

$-1861.979604$

$\begin{array}{lrrr}\mathrm{C} & 0.66451700 & 0.01693500 & 0.46465900 \\ \mathrm{~S} & 0.40131600 & -1.25430900 & 1.50945400 \\ \mathrm{~N} & -0.38913800 & 0.66060200 & -0.17667100 \\ \mathrm{H} & -0.22831300 & 1.59397800 & -0.53352800 \\ \mathrm{~N} & 1.87098100 & 0.53134000 & 0.21100700 \\ \mathrm{C} & 2.20374500 & 1.52833600 & -0.74569200 \\ \mathrm{C} & 1.86552300 & 1.37893000 & -2.09008300 \\ \mathrm{C} & 2.96337700 & 2.61892900 & -0.33124500 \\ \mathrm{C} & 2.27339100 & 2.33466200 & -3.01082100 \\ \mathrm{H} & 1.31481300 & 0.50286100 & -2.41337400 \\ \mathrm{C} & 3.38074500 & 3.56076500 & -1.26169300\end{array}$

$\begin{array}{lrrr}\mathrm{H} & 3.23392700 & 2.71088000 & 0.71407700 \\ \mathrm{C} & 3.03269800 & 3.42424600 & -2.60042300 \\ \mathrm{H} & 3.36088900 & 4.15996600 & -3.32454400 \\ \mathrm{H} & 3.39970000 & -1.58123200 & -0.70387100 \\ \mathrm{C} & 4.63327300 & -1.61193200 & 1.05164600 \\ \mathrm{C} & 4.46378200 & -1.61332700 & -0.45743200 \\ \mathrm{C} & 5.24361800 & -0.46020900 & -1.11891100 \\ \mathrm{C} & 6.44875600 & -0.05163300 & -0.25505900 \\ \mathrm{C} & 6.94957900 & -1.23919700 & 0.55214500 \\ \mathrm{H} & 5.57981600 & -0.78371500 & -2.10749600 \\ \mathrm{H} & 4.59559300 & 0.40360800 & -1.27781000\end{array}$




\begin{tabular}{|c|c|c|c|c|c|c|c|}
\hline $\mathrm{H}$ & 7.26280000 & 0.33279600 & -0.87405400 & $\mathrm{H}$ & -5.39968400 & 0.25245100 & -0.05125300 \\
\hline $\mathrm{H}$ & 6.15881300 & 0.74094800 & 0.43879100 & $\mathrm{C}$ & -5.88725800 & 0.85106200 & 1.94624000 \\
\hline $\mathrm{H}$ & 7.27033400 & -2.04637700 & -0.11972300 & $\mathrm{C}$ & -6.03226000 & 2.27568700 & -0.15756000 \\
\hline $\mathrm{H}$ & 7.80015000 & -0.96673000 & 1.17753700 & $\mathrm{H}$ & -4.24543600 & 3.12429300 & 1.72135900 \\
\hline $\mathrm{O}$ & 5.95923300 & -1.75244900 & 1.44777600 & $\mathrm{C}$ & -2.38624000 & 2.35304600 & 2.41919700 \\
\hline $\mathrm{H}$ & 4.08917500 & -2.43800900 & 1.52273200 & $\mathrm{C}$ & -2.78167200 & 3.61195900 & 0.24426200 \\
\hline $\mathrm{H}$ & 4.84025200 & -2.57723100 & -0.81071500 & $\mathrm{H}$ & -5.12623600 & -0.97652900 & -2.06444400 \\
\hline $\mathrm{C}$ & 4.02915900 & -0.32434500 & 2.96267300 & $\mathrm{H}$ & -4.93282100 & -2.66230600 & -2.56717000 \\
\hline $\mathrm{H}$ & 3.36381600 & -1.10170600 & 3.35508300 & $\mathrm{H}$ & -6.30803300 & -2.19930900 & -1.56599400 \\
\hline $\mathrm{H}$ & 3.62717600 & 0.65535800 & 3.22101600 & $\mathrm{H}$ & -4.27803600 & -4.46246500 & -0.73050000 \\
\hline $\mathrm{H}$ & 5.02697700 & -0.43843100 & 3.39002300 & $\mathrm{H}$ & -4.05381700 & -3.89909000 & 0.93886900 \\
\hline $\mathrm{O}$ & 4.08561000 & -0.38844200 & 1.54473400 & $\mathrm{H}$ & -5.66406200 & -3.91890800 & 0.20855700 \\
\hline $\mathrm{H}$ & 2.65442500 & 0.14726800 & 0.76500600 & $\mathrm{H}$ & -0.63442900 & -3.20503000 & 0.02715800 \\
\hline $\mathrm{H}$ & 3.98034200 & 4.40346100 & -0.93891700 & $\mathrm{H}$ & -1.95597000 & -4.38438300 & 0.01761300 \\
\hline $\mathrm{H}$ & 2.01905800 & 2.21311000 & -4.05709700 & $\mathrm{H}$ & -0.73294900 & -4.42483700 & -1.25621700 \\
\hline $\mathrm{C}$ & -1.67902400 & 0.23200600 & -0.08895100 & $\mathrm{H}$ & -1.82721500 & -1.27650500 & -2.99717400 \\
\hline $\mathrm{C}$ & -2.63164900 & -0.73479200 & -0.29718100 & $\mathrm{H}$ & -0.46679100 & -1.45904200 & -1.86079700 \\
\hline $\mathrm{C}$ & -2.97674400 & 0.54240900 & 0.21144900 & $\mathrm{H}$ & -0.74465700 & -2.66997200 & -3.10660800 \\
\hline $\mathrm{N}$ & -3.01928300 & -1.92821800 & -0.66776500 & $\mathrm{H}$ & -5.80170100 & 1.71507600 & 2.61048000 \\
\hline $\mathrm{N}$ & -3.85389000 & 1.41160100 & 0.65147000 & $\mathrm{H}$ & -6.94803000 & 0.60698100 & 1.85949500 \\
\hline $\mathrm{C}$ & -4.44344600 & -2.29910900 & -0.47683000 & $\mathrm{H}$ & -5.37555500 & 0.00740300 & 2.41556500 \\
\hline $\mathrm{C}$ & -2.13251200 & -2.80871500 & -1.47786600 & $\mathrm{H}$ & -5.59676300 & 2.45165600 & -1.14339900 \\
\hline $\mathrm{C}$ & -5.30645600 & 1.14594400 & 0.56727000 & $\mathrm{H}$ & -7.08170800 & 2.00644000 & -0.29111400 \\
\hline $\mathrm{C}$ & -3.36024900 & 2.65880900 & 1.28546700 & $\mathrm{H}$ & -6.00560400 & 3.21091100 & 0.40693300 \\
\hline $\mathrm{C}$ & -5.24638500 & -2.01516900 & -1.74412300 & $\mathrm{H}$ & -2.13135500 & 3.27740400 & 2.94106800 \\
\hline $\mathrm{H}$ & -4.79948600 & -1.64911700 & 0.32519900 & $\mathrm{H}$ & -2.82834600 & 1.66237000 & 3.13984300 \\
\hline $\mathrm{C}$ & -4.60623900 & -3.73276200 & 0.01290500 & $\mathrm{H}$ & -1.45584700 & 1.91237800 & 2.05112800 \\
\hline $\mathrm{C}$ & -1.31876400 & -3.76429800 & -0.61251400 & $\mathrm{H}$ & -1.89173300 & 3.18742000 & -0.22965600 \\
\hline $\mathrm{H}$ & -2.82034000 & -3.38789200 & -2.09900000 & $\mathrm{H}$ & -3.51087800 & 3.83698400 & -0.53566500 \\
\hline $\mathrm{C}$ & -1.24470200 & -1.99279100 & -2.41216400 & $\mathrm{H}$ & -2.48412300 & 4.54894300 & 0.71877100 \\
\hline
\end{tabular}

iii. Z,Z Conformation

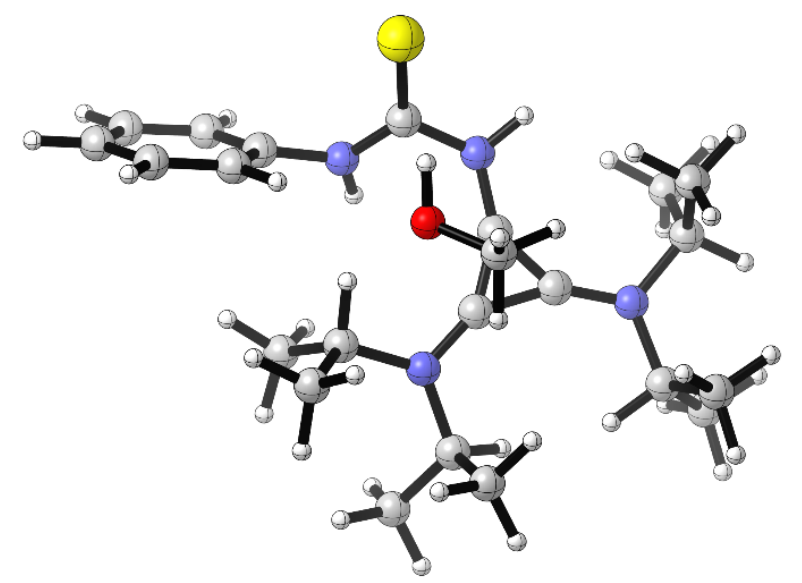

Figure S30. Optimized Structure of Z,Z-1-An-INT ${ }_{1}$. 
- Thermochemistry -

(0 imaginary frequencies)

Zero-point correction=

Thermal correction to Energy=

Thermal correction to Enthalpy=

Thermal correction to Gibbs Free Energy=

Sum of electronic and zero-point Energies=

Sum of electronic and thermal Energies=

Sum of electronic and thermal Enthalpies=

Sum of electronic and thermal Free Energies=
0.603114 (Hartree/Particle)

0.637273

0.638217

0.538294

$-1591.457405$

$-1591.423246$

$-1591.422301$

$-1591.522225$ $\begin{array}{rrc}-3.69476400 & 1.37739200 & -0.20284900 \\ -5.23296100 & 0.54636500 & -1.42193100 \\ -4.97294300 & 0.27731200 & 1.09540700 \\ -4.79487300 & -1.98371200 & -0.57595000 \\ -3.23256300 & -2.67905400 & -1.84054000 \\ -3.30879900 & -2.81027700 & 0.70591300 \\ -2.39987000 & 2.20801500 & 1.55857500 \\ -1.28410400 & 3.56294700 & 1.77788000 \\ -2.85648000 & 3.83045200 & 1.02059800 \\ -0.13425700 & 4.61793500 & -0.38150400 \\ -0.54169200 & 3.91281000 & -1.95794000 \\ -1.74778300 & 4.82530500 & -1.04693700 \\ 1.02287700 & 3.80283400 & 1.19764300 \\ 0.76447600 & 2.32977300 & 2.16770200 \\ 2.35813800 & 2.69994800 & 1.51466800 \\ -5.85960700 & -0.34469300 & -1.50889900 \\ -5.90051300 & 1.39868300 & -1.28048500 \\ -4.69504500 & 0.68348800 & -2.36226000 \\ -5.57503400 & -0.63477900 & 1.12076500 \\ -4.25110900 & 0.23895100 & 1.91490000 \\ -5.64607500 & 1.11890300 & 1.27048400 \\ -3.65125500 & -3.68353500 & -1.92564200 \\ -3.55779000 & -2.09869200 & -2.70603400 \\ -2.14369800 & -2.77246600 & -1.88344200 \\ -2.22286800 & -2.87221700 & 0.82516000 \\ -3.71556300 & -2.34393400 & 1.60509900 \\ -3.69113800 & -3.83104500 & 0.64690000 \\ 5.40321800 & 0.71502600 & 1.84092500 \\ 6.39236100 & -0.07416700 & -2.25477000 \\ 1.33762600 & 0.91839500 & 0.29970400 \\ 1.81501900 & 2.40544000 & -1.15620400 \\ 1.67400600 & 3.46729100 & -1.35813500 \\ 2.87835600 & 2.23908200 & -0.96897200 \\ 1.53922700 & 1.85248100 & -2.05940900\end{array}$ 


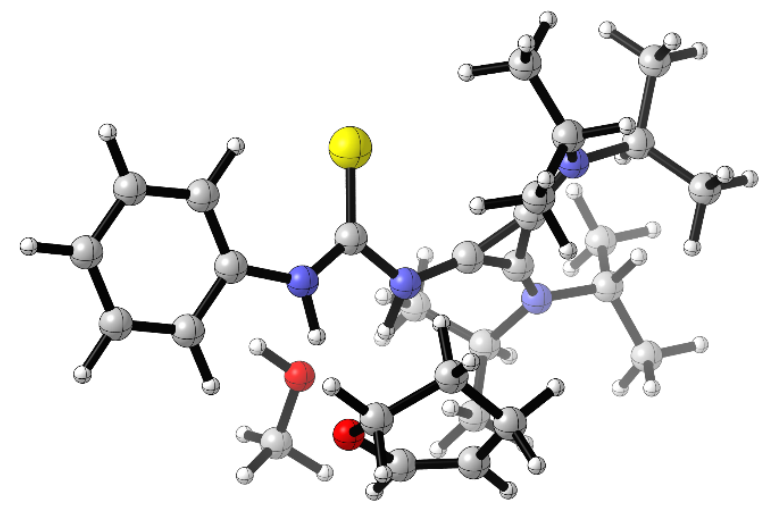

Figure S31. Optimized Structure of Z,Z-1-An-INT 2.

- Thermochemistry -

(0 imaginary frequencies)

Zero-point correction $=$

Thermal correction to Energy=

0.728586 (Hartree/Particle)

Thermal correction to Enthalpy=

0.769970

Thermal correction to Gibbs Free Energy=

0.770914

Sum of electronic and zero-point Energies=

0.652420

Sum of electronic and thermal Energies=

$-1861.889420$

Sum of electronic and thermal Enthalpies=

$-1861.848036$

Sum of electronic and thermal Free Energies=

$-1861.847092$

$-1861.965586$

$\begin{array}{lrrr}\mathrm{C} & -1.39131800 & 0.71678400 & -0.75545300 \\ \mathrm{~S} & -0.99527400 & 1.97677900 & -1.75184100 \\ \mathrm{~N} & -0.40841200 & -0.16574100 & -0.26344700 \\ \mathrm{H} & -0.68246900 & -1.14942000 & -0.27401200 \\ \mathrm{~N} & -2.59961300 & 0.32172300 & -0.31857600 \\ \mathrm{C} & -3.91566400 & 0.70253000 & -0.67753400 \\ \mathrm{C} & -4.25293400 & 1.86499900 & -1.36493300 \\ \mathrm{C} & -4.91833100 & -0.18385200 & -0.26782800 \\ \mathrm{C} & -5.59112100 & 2.11942400 & -1.64599300 \\ \mathrm{H} & -3.48816500 & 2.56047800 & -1.67241900 \\ \mathrm{C} & -6.24605000 & 0.08825000 & -0.55108900 \\ \mathrm{H} & -4.64795400 & -1.08130300 & 0.28060300 \\ \mathrm{C} & -6.58884400 & 1.24215000 & -1.24739100 \\ \mathrm{H} & -7.62691600 & 1.45571400 & -1.47089300 \\ \mathrm{H} & -2.58622300 & -0.45083700 & 0.34871000 \\ \mathrm{C} & -1.60234300 & -2.35665000 & 2.10395700 \\ \mathrm{C} & -0.55396400 & -2.06194800 & 2.86669800 \\ \mathrm{C} & -0.44032600 & -0.76310600 & 3.61367700 \\ \mathrm{C} & -1.53097800 & 0.21034600 & 3.15961300 \\ \mathrm{C} & -2.83682400 & -0.53492400 & 2.94193500 \\ \mathrm{H} & -0.51633800 & -0.94922700 & 4.69077600 \\ \mathrm{H} & 0.54970000 & -0.32372500 & 3.45261900 \\ \mathrm{H} & -1.68160900 & 1.00765000 & 3.89070200 \\ \mathrm{H} & -1.24325400 & 0.68192900 & 2.21716100 \\ \mathrm{H} & -3.17007200 & -1.02820400 & 3.86087600 \\ \mathrm{H} & -3.63085100 & 0.12076200 & 2.58379700 \\ \mathrm{O} & -2.68702200 & -1.54327900 & 1.92912400\end{array}$

$\begin{array}{lrrr}\mathrm{H} & -1.70083100 & -3.27742200 & 1.54382700 \\ \mathrm{H} & 0.23131100 & -2.80299800 & 2.95770200 \\ \mathrm{C} & -2.17619800 & -3.82550100 & -1.13936300 \\ \mathrm{H} & -3.12099900 & -3.98063100 & -0.60919800 \\ \mathrm{H} & -1.38027400 & -4.34008900 & -0.60126700 \\ \mathrm{H} & -2.24839500 & -4.25431800 & -2.14280700 \\ \mathrm{O} & -1.82352400 & -2.44947600 & -1.18540300 \\ \mathrm{H} & -2.47565700 & -1.97761900 & -1.70771000 \\ \mathrm{H} & -5.84898600 & 3.02531200 & -2.18178500 \\ \mathrm{H} & -7.01373000 & -0.60469500 & -0.22784500 \\ \mathrm{C} & 0.91587900 & 0.08585900 & -0.30367700 \\ \mathrm{C} & 2.16480900 & -0.45772800 & -0.45558800 \\ \mathrm{C} & 2.01150800 & 0.89822900 & -0.11665000 \\ \mathrm{~N} & 2.90754500 & -1.51182000 & -0.72345600 \\ \mathrm{~N} & 2.59637700 & 2.04580800 & 0.15928200 \\ \mathrm{C} & 4.37588600 & -1.46772200 & -0.56603900 \\ \mathrm{C} & 2.26294300 & -2.73403800 & -1.25687300 \\ \mathrm{C} & 4.07421700 & 2.10191700 & 0.04585300 \\ \mathrm{C} & 1.88926300 & 3.15043400 & 0.86052700 \\ \mathrm{C} & 4.87705000 & -2.60186700 & 0.32369300 \\ \mathrm{H} & 4.58090200 & -0.53626800 & -0.04003100 \\ \mathrm{C} & 5.07056800 & -1.42469000 & -1.92377400 \\ \mathrm{C} & 1.42224500 & -2.43068900 & -2.49351300 \\ \mathrm{H} & 3.08998100 & -3.37338800 & -1.56978700 \\ \mathrm{C} & 1.48449700 & -3.46429600 & -0.16626800 \\ \mathrm{H} & 4.33106800 & 1.33669000 & -0.68903200 \\ \mathrm{C} & 4.57029000 & 3.42812200 & -0.51815700\end{array}$




$\begin{array}{lrrrrrrr}\mathrm{C} & 4.73533900 & 1.76013100 & 1.38063300 & \mathrm{H} & 0.65666900 & -2.85788700 & 0.21136600 \\ \mathrm{H} & 2.65221300 & 3.58930800 & 1.50927200 & \mathrm{H} & 1.06704200 & -4.39059500 & -0.56748600 \\ \mathrm{C} & 1.38207100 & 4.22576900 & -0.09521900 & \mathrm{H} & 4.37730100 & 4.26230600 & 0.15987800 \\ \mathrm{C} & 0.77528400 & 2.62535900 & 1.75745300 & \mathrm{H} & 5.65094900 & 3.36515500 & -0.66070700 \\ \mathrm{H} & 4.36962200 & -2.59426400 & 1.29064500 & \mathrm{H} & 4.11093000 & 3.64290100 & -1.48398500 \\ \mathrm{H} & 4.73531800 & -3.58230900 & -0.13735500 & \mathrm{H} & 4.36159700 & 0.81339200 & 1.78087000 \\ \mathrm{H} & 5.94738100 & -2.47669700 & 0.49898200 & \mathrm{H} & 5.81712500 & 1.67603600 & 1.25455400 \\ \mathrm{H} & 4.89775300 & -2.34360300 & -2.49027600 & \mathrm{H} & 4.55013200 & 2.53713700 & 2.12647400 \\ \mathrm{H} & 4.71170400 & -0.58260500 & -2.52051900 & \mathrm{H} & 0.94017000 & 5.04173100 & 0.48185000 \\ \mathrm{H} & 6.14905500 & -1.31778300 & -1.78983900 & \mathrm{H} & 2.18295600 & 4.64099300 & -0.70564000 \\ \mathrm{H} & 0.51696100 & -1.87067200 & -2.25052900 & \mathrm{H} & 0.61703000 & 3.81673000 & -0.75492100 \\ \mathrm{H} & 2.00191400 & -1.86753900 & -3.22760600 & \mathrm{H} & -0.07274600 & 2.27409300 & 1.16704400 \\ \mathrm{H} & 1.10416800 & -3.36809700 & -2.95482400 & \mathrm{H} & 1.12482700 & 1.81816000 & 2.40646400 \\ \mathrm{H} & 2.13150600 & -3.71712000 & 0.67583700 & \mathrm{H} & 0.41393600 & 3.43685400 & 2.39136000\end{array}$

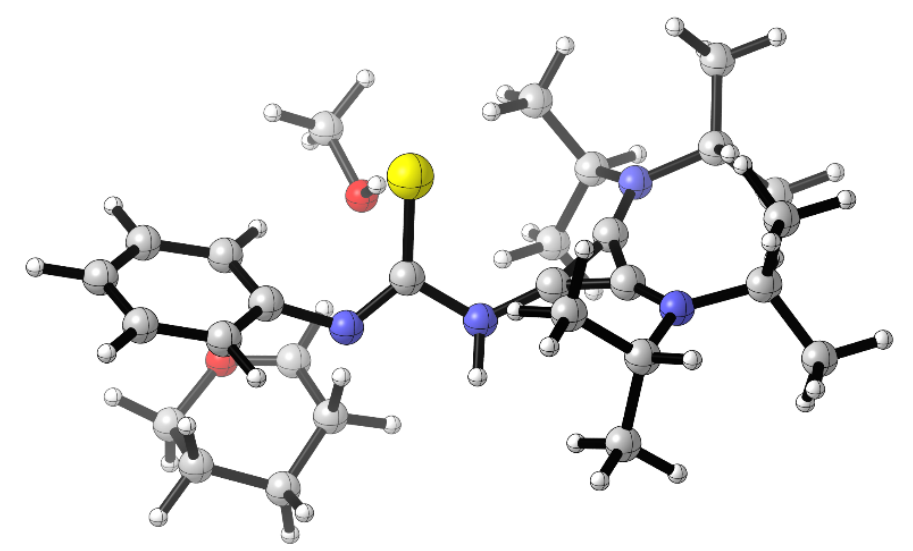

Figure S32. Optimized Structure of Z,Z-1-An-TS . $_{\text {. }}$

- Thermochemistry -

$(1$ imaginary frequency $=-373.31 \mathrm{~Hz})$

Zero-point correction $=$

Thermal correction to Energy=

0.722930 (Hartree/Particle)

Thermal correction to Enthalpy=

$$
0.763533
$$

0.764478

Thermal correction to Gibbs Free Energy=

0.648222

Sum of electronic and zero-point Energies=

$-1861.842948$

Sum of electronic and thermal Energies=

$-1861.802345$

Sum of electronic and thermal Enthalpies=

$-1861.801401$

Sum of electronic and thermal Free Energies=

$-1861.917656$

$\begin{array}{cccc}\mathrm{C} & 0.80654200 & -0.47955800 & -0.56154100 \\ \mathrm{~S} & 0.39578500 & 0.41804800 & -1.93953800 \\ \mathrm{~N} & -0.20273100 & -0.74495500 & 0.40781400 \\ \mathrm{H} & -0.04743300 & -1.61638400 & 0.89644700 \\ \mathrm{~N} & 1.93502200 & -1.03121600 & -0.20237400 \\ \mathrm{C} & 3.08899900 & -1.03721200 & -1.01633400 \\ \mathrm{C} & 3.62453400 & -2.27293300 & -1.38098100 \\ \mathrm{C} & 3.79411500 & 0.12723100 & -1.31837200 \\ \mathrm{C} & 4.84768800 & -2.34289700 & -2.03229100 \\ \mathrm{H} & 3.07319300 & -3.17356900 & -1.13626200 \\ \mathrm{C} & 5.02580800 & 0.05187100 & -1.95757800\end{array}$

$\begin{array}{lrrr}\mathrm{H} & 3.37751900 & 1.08777600 & -1.04470900 \\ \mathrm{C} & 5.55870300 & -1.18030500 & -2.31417400 \\ \mathrm{H} & 6.51648500 & -1.23645800 & -2.81777300 \\ \mathrm{H} & 2.45452400 & -0.54260000 & 1.15812200 \\ \mathrm{C} & 3.58800100 & 1.01846400 & 1.78962800 \\ \mathrm{C} & 2.97701900 & -0.18156700 & 2.21216900 \\ \mathrm{C} & 3.89470600 & -1.29796400 & 2.68232900 \\ \mathrm{C} & 5.19980800 & -1.23118700 & 1.88651800 \\ \mathrm{C} & 5.80241900 & 0.15364200 & 1.97401400 \\ \mathrm{H} & 4.09581200 & -1.20548800 & 3.75403000 \\ \mathrm{H} & 3.40845000 & -2.26248100 & 2.52538300\end{array}$




\begin{tabular}{|c|c|c|c|c|c|c|c|}
\hline $\mathrm{H}$ & 5.93316200 & -1.94281700 & 2.27146300 & $\mathrm{H}$ & -5.22374500 & -0.50833600 & 0.50738400 \\
\hline $\mathrm{H}$ & 5.01893000 & -1.48234000 & 0.83862100 & $\mathrm{C}$ & -5.76185300 & -1.73834800 & -1.16181600 \\
\hline $\mathrm{H}$ & 6.14125400 & 0.40370500 & 2.98139500 & $\mathrm{C}$ & -5.79105100 & -2.39164100 & 1.29730600 \\
\hline $\mathrm{H}$ & 6.61673100 & 0.30347800 & 1.26933300 & $\mathrm{H}$ & -4.04458800 & -3.78530500 & -0.24640500 \\
\hline $\mathrm{O}$ & 4.84146000 & 1.20470800 & 1.62019000 & $\mathrm{C}$ & -2.24865600 & -3.26895100 & -1.26544100 \\
\hline $\mathrm{H}$ & 2.98875200 & 1.87095500 & 1.46369600 & $\mathrm{C}$ & -2.50317900 & -3.71027300 & 1.22864300 \\
\hline $\mathrm{H}$ & 2.06704700 & 0.00878100 & 2.78433500 & $\mathrm{H}$ & -4.89392200 & 1.37510300 & 1.88036100 \\
\hline $\mathrm{C}$ & 2.36729200 & 3.79258400 & -0.91974900 & $\mathrm{H}$ & -4.75084600 & 3.12996000 & 1.71032600 \\
\hline $\mathrm{H}$ & 3.06732900 & 3.45440100 & -1.69223900 & $\mathrm{H}$ & -6.16185800 & 2.30000900 & 1.05591400 \\
\hline $\mathrm{H}$ & 2.88712600 & 4.48212900 & -0.25402500 & $\mathrm{H}$ & -4.31991500 & 4.13691200 & -0.69768700 \\
\hline $\mathrm{H}$ & 1.54409700 & 4.32839300 & -1.40409300 & $\mathrm{H}$ & -4.15048700 & 3.00593900 & -2.05654200 \\
\hline $\mathrm{O}$ & 1.89305300 & 2.71177300 & -0.13365100 & $\mathrm{H}$ & -5.72018800 & 3.23993000 & -1.27516400 \\
\hline $\mathrm{H}$ & 1.42076800 & 2.09996200 & -0.72319100 & $\mathrm{H}$ & -0.73370200 & 2.77608700 & -1.26945200 \\
\hline $\mathrm{H}$ & 5.25142400 & -3.30787300 & -2.31643600 & $\mathrm{H}$ & -2.06681700 & 3.91394200 & -1.51492800 \\
\hline $\mathrm{H}$ & 5.56547500 & 0.96436200 & -2.18529700 & $\mathrm{H}$ & -0.70829000 & 4.36224300 & -0.47349500 \\
\hline $\mathrm{C}$ & -1.50480500 & -0.38862800 & 0.24680900 & $\mathrm{H}$ & -1.57002000 & 2.10919900 & 2.38631700 \\
\hline $\mathrm{C}$ & -2.49231300 & 0.56320900 & 0.15104700 & $\mathrm{H}$ & -0.29026900 & 1.87551300 & 1.16580000 \\
\hline $\mathrm{C}$ & -2.80594900 & -0.81142400 & 0.18018900 & $\mathrm{H}$ & -0.54105600 & 3.45869600 & 1.87615100 \\
\hline $\mathrm{N}$ & -2.92309500 & 1.80022000 & 0.07711100 & $\mathrm{H}$ & -5.66815400 & -2.77066800 & -1.50938500 \\
\hline $\mathrm{N}$ & -3.66837000 & -1.80400400 & 0.15474600 & $\mathrm{H}$ & -6.82681300 & -1.50082600 & -1.11644500 \\
\hline $\mathrm{C}$ & -4.37244400 & 2.03005200 & -0.12939200 & $\mathrm{H}$ & -5.29267400 & -1.08627400 & -1.90265800 \\
\hline $\mathrm{C}$ & -2.04301300 & 2.94394300 & 0.43573200 & $\mathrm{H}$ & -5.31519400 & -2.22784700 & 2.26642500 \\
\hline $\mathrm{C}$ & -5.12303100 & -1.55318200 & 0.21135400 & $\mathrm{H}$ & -6.84172700 & -2.10690000 & 1.38052100 \\
\hline $\mathrm{C}$ & -3.15871700 & -3.18148900 & -0.04347900 & $\mathrm{H}$ & -5.76131700 & -3.46031500 & 1.07118200 \\
\hline $\mathrm{C}$ & -5.08358700 & 2.21740200 & 1.20934200 & $\mathrm{H}$ & -2.00512400 & -4.31439300 & -1.46477500 \\
\hline $\mathrm{H}$ & -4.74100500 & 1.11941800 & -0.60572400 & $\mathrm{H}$ & -2.73989800 & -2.85692500 & -2.14894300 \\
\hline $\mathrm{C}$ & -4.64474900 & 3.17412400 & -1.09862600 & $\mathrm{H}$ & -1.30789100 & -2.73212300 & -1.11960700 \\
\hline $\mathrm{C}$ & -1.34966100 & 3.53537000 & -0.78678400 & $\mathrm{H}$ & -1.62583900 & -3.11438700 & 1.49835500 \\
\hline $\mathrm{H}$ & -2.72329700 & 3.69147600 & 0.85268800 & $\mathrm{H}$ & -3.19846000 & -3.68895200 & 2.06948000 \\
\hline $\mathrm{C}$ & -1.05621700 & 2.56026800 & 1.53308400 & $\mathrm{H}$ & -2.17046100 & -4.73964300 & 1.08109000 \\
\hline
\end{tabular}

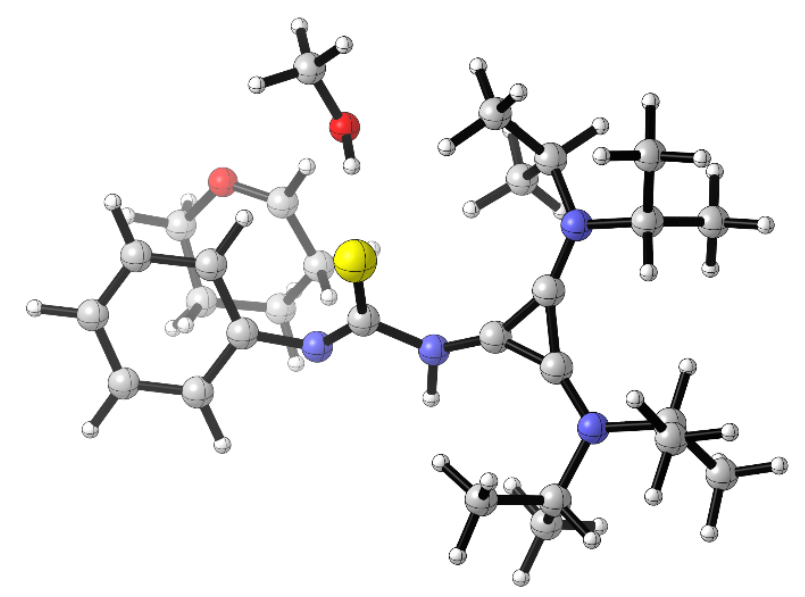

Figure S33. Optimized Structure of Z,Z-1-An-INT 3 .

- Thermochemistry -

(0 imaginary frequencies)

Zero-point correction $=$

Thermal correction to Energy=

0.726072 (Hartree/Particle)

Thermal correction to Enthalpy=

0.767283

Thermal correction to Gibbs Free Energy $=\quad 0.650427$

0.768227 
Sum of electronic and zero-point Energies= Sum of electronic and thermal Energies $=$ Sum of electronic and thermal Enthalpies= Sum of electronic and thermal Free Energies=
$-1861.842898$

$-1861.801687$

$-1861.800743$

$-1861.918543$

$\begin{array}{lrrr}\mathrm{C} & 0.73303400 & -0.61896700 & -0.56327800 \\ \mathrm{~S} & 0.43270900 & 0.48006600 & -1.83895300 \\ \mathrm{~N} & -0.36933000 & -0.95076900 & 0.28300700 \\ \mathrm{H} & -0.26507300 & -1.86154500 & 0.70902200 \\ \mathrm{~N} & 1.77760300 & -1.30230500 & -0.21516400 \\ \mathrm{C} & 2.99692900 & -1.29656600 & -0.89759000 \\ \mathrm{C} & 3.64972300 & -2.53099500 & -1.02440500 \\ \mathrm{C} & 3.66267100 & -0.14725200 & -1.33895900 \\ \mathrm{C} & 4.91532100 & -2.61584500 & -1.58110200 \\ \mathrm{H} & 3.13521300 & -3.41892600 & -0.67571100 \\ \mathrm{C} & 4.94327900 & -0.23572300 & -1.87903000 \\ \mathrm{H} & 3.16937000 & 0.81284100 & -1.28117400 \\ \mathrm{C} & 5.57675100 & -1.46358200 & -2.00415900 \\ \mathrm{H} & 6.56498000 & -1.53028900 & -2.44372800 \\ \mathrm{H} & 2.61515200 & -0.46306100 & 1.44411100 \\ \mathrm{C} & 3.91278100 & 1.11531700 & 1.37559200 \\ \mathrm{C} & 3.32294500 & 0.03110600 & 2.14956900 \\ \mathrm{C} & 4.32080900 & -0.95499200 & 2.75008400 \\ \mathrm{C} & 5.53283400 & -1.06918600 & 1.82727400 \\ \mathrm{C} & 6.14838500 & 0.28974000 & 1.60322000 \\ \mathrm{H} & 4.63569600 & -0.61886700 & 3.74247100 \\ \mathrm{H} & 3.84194500 & -1.92622500 & 2.87327300 \\ \mathrm{H} & 6.29918900 & -1.71569100 & 2.25966900 \\ \mathrm{H} & 5.24366400 & -1.50150100 & 0.86614300 \\ \mathrm{H} & 6.58431900 & 0.72475200 & 2.50397700 \\ \mathrm{H} & 6.87594000 & 0.30616400 & 0.79557800 \\ \mathrm{O} & 5.14276600 & 1.28101600 & 1.16282400 \\ \mathrm{H} & 3.27361400 & 1.88310100 & 0.91402700 \\ \mathrm{H} & 2.65469500 & 0.50305100 & 2.88144100 \\ \mathrm{C} & 2.41813800 & 3.73981300 & -1.18536800 \\ \mathrm{H} & 2.97773400 & 3.28163900 & -2.00869600 \\ \mathrm{H} & 3.06293300 & 4.45461900 & -0.67290800 \\ \mathrm{H} & 1.56260500 & 4.27999300 & -1.60356800 \\ \mathrm{O} & 2.00159600 & 2.77286700 & -0.23400900 \\ \mathrm{H} & 1.45628900 & 2.11407000 & -0.70649100 \\ \mathrm{H} & 5.39233300 & -3.58385400 & -1.68508300 \\ \mathrm{H} & 5.43736200 & 0.66729400 & -2.22150800 \\ \mathrm{C} & -1.62680800 & -0.47914300 & 0.16126500 \\ \mathrm{C} & -2.54874000 & 0.54330900 & 0.11356400 \\ \mathrm{~N} & -2.96151600 & -0.79786900 & 0.15335200 \\ & -2.89656000 & 1.81300700 & 0.07129500 \\ & & & \\ \mathrm{H} & & \end{array}$

$\begin{array}{rrc}-3.89352700 & -1.73045000 & 0.17161700 \\ -4.33726000 & 2.12730100 & -0.06765000 \\ -1.95155600 & 2.89236700 & 0.46018300 \\ -5.32208300 & -1.37773900 & 0.28479400 \\ -3.49529600 & -3.13991600 & -0.04484900 \\ -4.99117800 & 2.30006800 & 1.30291900 \\ -4.77156900 & 1.25686800 & -0.56347400 \\ -4.58879300 & 3.32158700 & -0.98036500 \\ -1.28570000 & 3.54348600 & -0.74780300 \\ -2.57810600 & 3.63718100 & 0.95922400 \\ -0.93348100 & 2.39736600 & 1.48243700 \\ -5.33734600 & -0.32673500 & 0.57348900 \\ -6.02973600 & -1.52650700 & -1.05906600 \\ -6.00426600 & -2.15819000 & 1.40482200 \\ -4.43208100 & -3.67907200 & -0.19545100 \\ -2.66059900 & -3.29772100 & -1.31277000 \\ -2.81344000 & -3.71367100 & 1.19390300 \\ -4.80826200 & 1.43252000 & 1.94293400 \\ -4.60679000 & 3.18517400 & 1.81605800 \\ -6.07068800 & 2.42588900 & 1.19273000 \\ -4.21178600 & 4.25234900 & -0.55032100 \\ -4.12957700 & 3.17313300 & -1.95875300 \\ -5.66515200 & 3.43910100 & -1.12134900 \\ -0.72280500 & 2.79645700 & -1.30698400 \\ -2.01427800 & 4.00325500 & -1.41497800 \\ -0.59661600 & 4.31877400 & -0.40454900 \\ -1.42754400 & 1.93015600 & 2.33867600 \\ -0.23341700 & 1.68778500 & 1.03890200 \\ -0.34844300 & 3.24632400 & 1.83920600 \\ -6.03212700 & -2.56681600 & -1.39565800 \\ -7.07001200 & -1.20475500 & -0.97465800 \\ -5.54139200 & -0.92162000 & -1.82705700 \\ -5.47893900 & -2.02301400 & 2.35260700 \\ -7.02713300 & -1.79631400 & 1.52743100 \\ -6.06181400 & -3.22770300 & 1.18796300 \\ -2.48937400 & -4.35795800 & -1.50934300 \\ -3.17503900 & -2.86768100 & -2.17432400 \\ -1.68343500 & -2.81585100 & -1.22516800 \\ -1.87910100 & -3.18802200 & 1.41186300 \\ -3.45806100 & -3.63428200 & 2.07104200 \\ -2.57152500 & -4.76678500 & 1.03702200\end{array}$




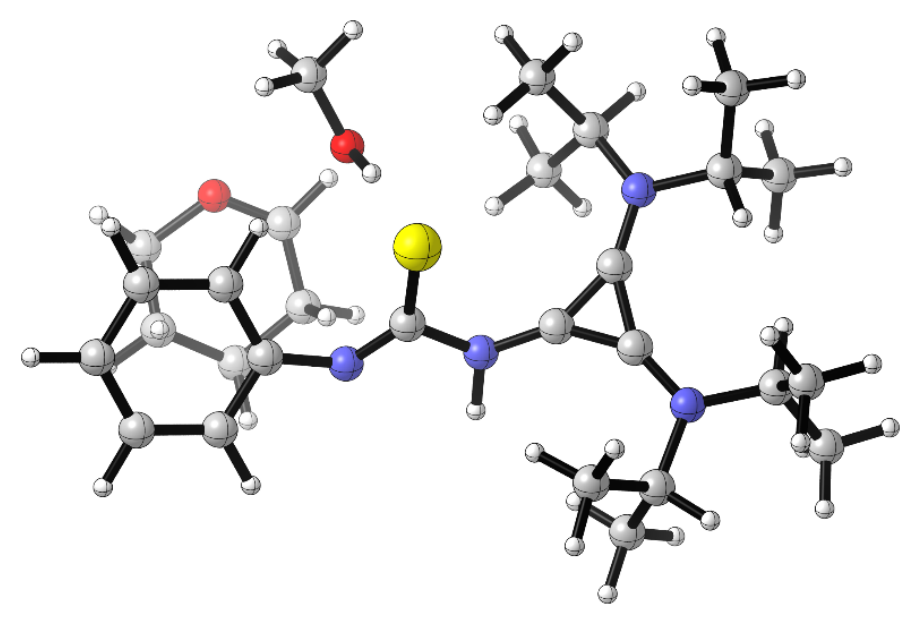

Figure S34. Optimized Structure of Z,Z-1-An-TS 2 .

- Thermochemistry -

$(1$ imaginary frequency $=-44.43 \mathrm{~Hz}$ )

Zero-point correction $=$

Thermal correction to Energy=

Thermal correction to Enthalpy=

Thermal correction to Gibbs Free Energy=

Sum of electronic and zero-point Energies=

Sum of electronic and thermal Energies $=$

Sum of electronic and thermal Enthalpies=

Sum of electronic and thermal Free Energies=
0.725305 (Hartree/Particle)

$$
0.765841
$$$$
0.766786
$$

0.651052

$-1861.839806$

$-1861.799269$

$-1861.798325$

$-1861.914058$

$\begin{array}{cccc}\mathrm{C} & 0.74472700 & -0.72568000 & -0.50560000 \\ \mathrm{~S} & 0.39921700 & 0.18647900 & -1.91249100 \\ \mathrm{~N} & -0.33261000 & -0.91310500 & 0.42091100 \\ \mathrm{H} & -0.23719300 & -1.77180000 & 0.94593000 \\ \mathrm{~N} & 1.79906200 & -1.34949600 & -0.08856000 \\ \mathrm{C} & 2.99336100 & -1.49325000 & -0.81002200 \\ \mathrm{C} & 3.66330000 & -2.71262400 & -0.64273700 \\ \mathrm{C} & 3.61023500 & -0.49797800 & -1.57653700 \\ \mathrm{C} & 4.89814700 & -2.93791100 & -1.23083800 \\ \mathrm{H} & 3.18528800 & -3.47896200 & -0.04299300 \\ \mathrm{C} & 4.85329200 & -0.72616200 & -2.15823800 \\ \mathrm{H} & 3.11599800 & 0.44881500 & -1.72211400 \\ \mathrm{C} & 5.50470400 & -1.94134900 & -1.99065100 \\ \mathrm{H} & 6.46826300 & -2.11572200 & -2.45470000 \\ \mathrm{H} & 2.55121700 & -0.18778500 & 1.35409100 \\ \mathrm{C} & 3.58389300 & 1.52593800 & 1.78362200 \\ \mathrm{C} & 3.17627400 & 0.19613100 & 2.20010300 \\ \mathrm{C} & 4.32139900 & -0.76708000 & 2.49901400 \\ \mathrm{C} & 5.47279500 & -0.50982800 & 1.52977800 \\ \mathrm{C} & 5.90308400 & 0.93524500 & 1.57415000 \\ \mathrm{H} & 4.65648500 & -0.64275300 & 3.53317400 \\ \mathrm{H} & 3.96167600 & -1.78972400 & 2.38677900 \\ \mathrm{H} & 6.34244400 & -1.12027200 & 1.78207100 \\ \mathrm{H} & 5.17776400 & -0.77283700 & 0.51396200\end{array}$

$\begin{array}{lrrr}\mathrm{H} & 6.39684500 & 1.21607600 & 2.50557600 \\ \mathrm{H} & 6.52256700 & 1.22794700 & 0.73013700 \\ \mathrm{O} & 4.75736600 & 1.87508100 & 1.49155200 \\ \mathrm{H} & 2.85886100 & 2.32685400 & 1.68526100 \\ \mathrm{H} & 2.45043700 & 0.30740900 & 3.01241500 \\ \mathrm{C} & 2.68660400 & 3.25562700 & -1.20477000 \\ \mathrm{H} & 3.20320200 & 2.76136700 & -2.03515200 \\ \mathrm{H} & 3.41261000 & 3.85322600 & -0.65016600 \\ \mathrm{H} & 1.92571000 & 3.92724800 & -1.61618000 \\ \mathrm{O} & 2.12085300 & 2.31802500 & -0.30632200 \\ \mathrm{H} & 1.54196200 & 1.71954800 & -0.82021900 \\ \mathrm{H} & 5.38958300 & -3.89510900 & -1.09782700 \\ \mathrm{H} & 5.30907700 & 0.05437100 & -2.75793700 \\ \mathrm{C} & -1.58829100 & -0.44969900 & 0.24229700 \\ \mathrm{C} & -2.48916500 & 0.57802400 & 0.07667400 \\ \mathrm{C} & -2.92803800 & -0.74690400 & 0.23373000 \\ \mathrm{~N} & -2.80836300 & 1.84336900 & -0.09895700 \\ \mathrm{~N} & -3.87991600 & -1.65479000 & 0.31049400 \\ \mathrm{C} & -4.23291600 & 2.17341300 & -0.33214100 \\ \mathrm{C} & -1.84974600 & 2.93471000 & 0.21203000 \\ \mathrm{C} & -5.30168500 & -1.26125000 & 0.36381900 \\ \mathrm{C} & -3.50847200 & -3.08547700 & 0.22165100 \\ \mathrm{C} & -4.93703500 & 2.51508400 & 0.98006900 \\ \mathrm{H} & -4.67129500 & 1.26288000 & -0.74581600\end{array}$




$\begin{array}{lrrrrrrr}\mathrm{C} & -4.41105600 & 3.26240100 & -1.38368100 & \mathrm{H} & -1.78947500 & 3.81475900 & -1.78013600 \\ \mathrm{C} & -1.10577700 & 3.42781600 & -1.02495400 & \mathrm{H} & -0.42209200 & 4.23107500 & -0.73878800 \\ \mathrm{H} & -2.47521700 & 3.74746700 & 0.59206000 & \mathrm{H} & -1.44542200 & 2.15896300 & 2.20332100 \\ \mathrm{C} & -0.89749900 & 2.53175600 & 1.33382100 & \mathrm{H} & -0.18584000 & 1.77360400 & 1.00255000 \\ \mathrm{H} & -5.29588600 & -0.19035700 & 0.56883000 & \mathrm{H} & -0.32186500 & 3.40767400 & 1.63881300 \\ \mathrm{C} & -5.98587400 & -1.49954300 & -0.97927400 & \mathrm{H} & -6.01727100 & -2.56421300 & -1.22621900 \\ \mathrm{C} & -6.02528100 & -1.93686800 & 1.52532200 & \mathrm{H} & -7.01594500 & -1.13785800 & -0.94644500 \\ \mathrm{H} & -4.45389700 & -3.61685300 & 0.10064600 & \mathrm{H} & -5.46139000 & -0.97874400 & -1.78423100 \\ \mathrm{C} & -2.65637200 & -3.36644300 & -1.01289400 & \mathrm{H} & -5.51238700 & -1.74733900 & 2.47056700 \\ \mathrm{C} & -2.85782400 & -3.56395000 & 1.51627200 & \mathrm{H} & -7.03901100 & -1.53840900 & 1.60173800 \\ \mathrm{H} & -4.80889000 & 1.72001300 & 1.71983500 & \mathrm{H} & -6.11015500 & -3.01719300 & 1.38511500 \\ \mathrm{H} & -4.54745100 & 3.44135900 & 1.41024400 & \mathrm{H} & -2.50200500 & -4.44225300 & -1.11668100 \\ \mathrm{H} & -6.00661400 & 2.65618600 & 0.80889900 & \mathrm{H} & -3.14836000 & -3.00184200 & -1.91671800 \\ \mathrm{H} & -4.01543600 & 4.22395800 & -1.04831400 & \mathrm{H} & -1.67208700 & -2.89539500 & -0.94912100 \\ \mathrm{H} & -3.92384500 & 2.98824900 & -2.32054200 & \mathrm{H} & -1.91309400 & -3.04365300 & 1.69979400 \\ \mathrm{H} & -5.47714600 & 3.39721400 & -1.57735600 & \mathrm{H} & -3.51156500 & -3.39244600 & 2.37326400 \\ \mathrm{H} & -0.53041800 & 2.61211900 & -1.46223300 & \mathrm{H} & -2.63985800 & -4.63210100 & 1.45553100\end{array}$

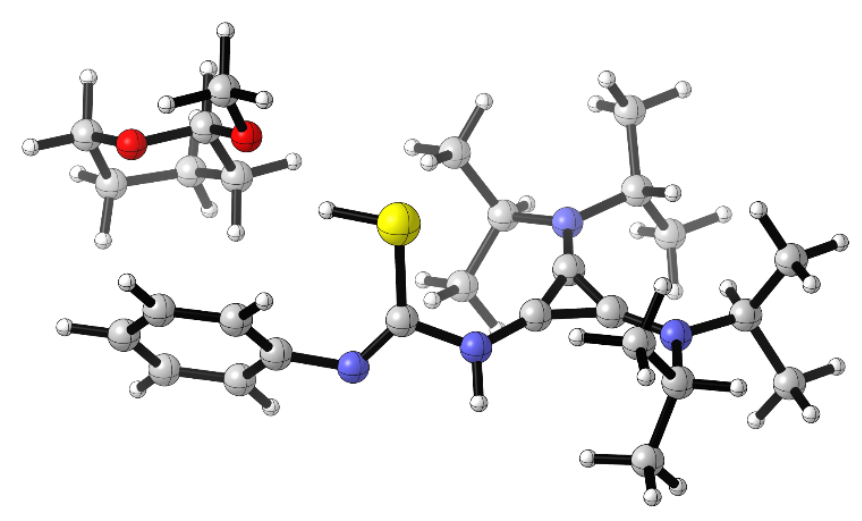

Figure S35. Optimized Structure of Z,Z-1-An-INT 4 .

- Thermochemistry -

(0 imaginary frequencies)

Zero-point correction $=$

Thermal correction to Energy=

Thermal correction to Enthalpy=

Thermal correction to Gibbs Free Energy $=0.651762$

Sum of electronic and zero-point Energies=

Sum of electronic and thermal Energies $=$

Sum of electronic and thermal Enthalpies=

Sum of electronic and thermal Free Energies=
0.726617 (Hartree/Particle)

$$
0.766621
$$

0.767565

$-1861.881249$

$-1861.841246$

$-1861.840301$

$-1861.956105$

$\begin{array}{cccc}\mathrm{C} & 0.19746700 & -1.51149600 & 0.13789000 \\ \mathrm{~S} & 0.55299600 & -0.62791400 & -1.36148500 \\ \mathrm{~N} & -1.20030400 & -1.69489800 & 0.28580200 \\ \mathrm{H} & -1.44218400 & -2.50098300 & 0.84589300 \\ \mathrm{~N} & 0.92757100 & -2.04071900 & 1.01500100 \\ \mathrm{C} & 2.33315300 & -2.08750200 & 0.99750900 \\ \mathrm{C} & 2.99047600 & -1.75385100 & 2.18263900 \\ \mathrm{C} & 3.07327400 & -2.51051700 & -0.10707300\end{array}$

$\begin{array}{lrrr}\mathrm{C} & 4.37510400 & -1.79971200 & 2.24606300 \\ \mathrm{H} & 2.40008400 & -1.46747900 & 3.04493700 \\ \mathrm{C} & 4.45824500 & -2.55560500 & -0.03301800 \\ \mathrm{H} & 2.56827500 & -2.81940100 & -1.01445300 \\ \mathrm{C} & 5.11324000 & -2.19453400 & 1.13606800 \\ \mathrm{H} & 6.19486500 & -2.22902300 & 1.18405900 \\ \mathrm{H} & 3.59062700 & 0.39856900 & 0.62350500 \\ \mathrm{C} & 4.41871600 & 1.09133400 & -1.20057700\end{array}$




\begin{tabular}{|c|c|c|c|c|c|c|c|}
\hline $\mathrm{C}$ & 3.79699100 & 1.36560400 & 0.15573100 & $\mathrm{C}$ & -0.02211700 & 2.81070400 & -0.08358800 \\
\hline $\mathrm{C}$ & 4.76810700 & 2.17307300 & 1.01993100 & $\mathrm{H}$ & -1.45829200 & 3.36026900 & 1.40231700 \\
\hline $\mathrm{C}$ & 6.13496900 & 1.48670700 & 1.05474700 & $\mathrm{C}$ & -0.58868400 & 1.52679100 & 2.02325200 \\
\hline $\mathrm{C}$ & 6.60789500 & 1.18862800 & -0.36283300 & $\mathrm{H}$ & -5.45558200 & 0.96011900 & 0.25777900 \\
\hline $\mathrm{H}$ & 4.87837800 & 3.18158900 & 0.60215500 & $\mathrm{C}$ & -6.47200500 & 0.21060600 & -1.47018400 \\
\hline $\mathrm{H}$ & 4.36782900 & 2.29036300 & 2.02987300 & $\mathrm{C}$ & -6.86539800 & -0.46519000 & 0.95102900 \\
\hline $\mathrm{H}$ & 6.87283600 & 2.11279600 & 1.56377100 & $\mathrm{H}$ & -5.95004100 & -2.43627100 & -0.69368700 \\
\hline $\mathrm{H}$ & 6.05823900 & 0.54437700 & 1.60502000 & $\mathrm{C}$ & -4.08858200 & -2.77446100 & -1.67417700 \\
\hline $\mathrm{H}$ & 6.80525700 & 2.12875000 & -0.90213900 & $\mathrm{C}$ & -4.60736700 & -3.19320000 & 0.78188100 \\
\hline $\mathrm{H}$ & 7.52493700 & 0.59953700 & -0.36745600 & $\mathrm{H}$ & -4.48333900 & 2.24737800 & 1.85002700 \\
\hline $\mathrm{O}$ & 5.64804800 & 0.42361700 & -1.07464900 & $\mathrm{H}$ & -3.58800800 & 3.76946800 & 1.94497900 \\
\hline $\mathrm{H}$ & 4.59018300 & 2.03971800 & -1.74856900 & $\mathrm{H}$ & -5.11508100 & 3.70651500 & 1.06782700 \\
\hline $\mathrm{H}$ & 2.84970900 & 1.89442500 & 0.01803600 & $\mathrm{H}$ & -2.39946600 & 4.68619100 & -0.20736000 \\
\hline $\mathrm{C}$ & 4.00105600 & -0.02540800 & -3.24250100 & $\mathrm{H}$ & -2.55584100 & 3.76249000 & -1.71668100 \\
\hline $\mathrm{H}$ & 4.91132700 & -0.62657500 & -3.21404800 & $\mathrm{H}$ & -3.95101300 & 4.60855400 & -1.03524500 \\
\hline $\mathrm{H}$ & 4.18969800 & 0.89350000 & -3.81050800 & $\mathrm{H}$ & 0.35854000 & 1.91657700 & -0.57759500 \\
\hline $\mathrm{H}$ & 3.19945000 & -0.58662800 & -3.72259700 & $\mathrm{H}$ & -0.38769200 & 3.49916000 & -0.84512700 \\
\hline $\mathrm{O}$ & 3.55435500 & 0.28311600 & -1.93175400 & $\mathrm{H}$ & 0.80520000 & 3.29556600 & 0.44085200 \\
\hline $\mathrm{H}$ & 1.88043500 & -0.42099300 & -1.17115800 & $\mathrm{H}$ & -1.38140600 & 1.25212100 & 2.72328900 \\
\hline $\mathrm{H}$ & 4.87831900 & -1.53530500 & 3.16911000 & $\mathrm{H}$ & -0.13523000 & 0.61585000 & 1.62700200 \\
\hline $\mathrm{H}$ & 5.02857500 & -2.86970900 & -0.89820300 & $\mathrm{H}$ & 0.18778100 & 2.05128100 & 2.58306300 \\
\hline $\mathrm{C}$ & -2.13045200 & -0.72327900 & 0.16777700 & $\mathrm{H}$ & -6.89243100 & -0.71918300 & -1.86245700 \\
\hline $\mathrm{C}$ & -2.53898800 & 0.59340300 & 0.17625000 & $\mathrm{H}$ & -7.28251500 & 0.94016700 & -1.41396200 \\
\hline $\mathrm{C}$ & -3.46587500 & -0.44989800 & 0.00067000 & $\mathrm{H}$ & -5.72809100 & 0.57622600 & -2.18218300 \\
\hline $\mathrm{N}$ & -2.32578200 & 1.89008500 & 0.26720700 & $\mathrm{H}$ & -6.39351800 & -0.58813500 & 1.92813900 \\
\hline $\mathrm{N}$ & -4.69019200 & -0.90030000 & -0.16127400 & $\mathrm{H}$ & -7.66011400 & 0.27729200 & 1.04682800 \\
\hline $\mathrm{C}$ & -3.47088900 & 2.79493400 & -0.00002700 & $\mathrm{H}$ & -7.33502600 & -1.41081400 & 0.66964500 \\
\hline $\mathrm{C}$ & -1.10886400 & 2.44345100 & 0.92105900 & $\mathrm{H}$ & -4.33297100 & -3.80817800 & -1.92612100 \\
\hline $\mathrm{C}$ & -5.85490100 & 0.00771400 & -0.09006800 & $\mathrm{H}$ & -4.32308600 & -2.14698900 & -2.53653100 \\
\hline $\mathrm{C}$ & -4.89071700 & -2.34041600 & -0.45137700 & $\mathrm{H}$ & -3.01174200 & -2.72592800 & -1.49107100 \\
\hline $\mathrm{C}$ & -4.20484800 & 3.14614400 & 1.29298800 & $\mathrm{H}$ & -3.55778700 & -3.12345900 & 1.08097500 \\
\hline $\mathrm{H}$ & -4.13870900 & 2.22071100 & -0.64597700 & $\mathrm{H}$ & -5.22264000 & -2.88285600 & 1.62797900 \\
\hline $\mathrm{C}$ & -3.05696600 & 4.03350800 & -0.78606900 & $\mathrm{H}$ & -4.81718500 & -4.24326400 & 0.56994300 \\
\hline
\end{tabular}

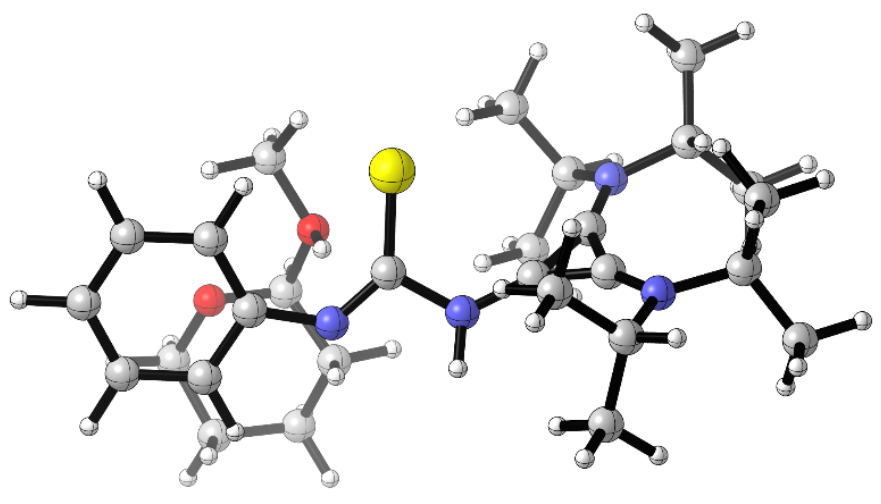

Figure S36. Optimized Structure of Z,Z-1-An-TS 3 -PA.

- Thermochemistry -

$(1$ imaginary frequency $=-111.33 \mathrm{~Hz})$

Zero-point correction $=$

Thermal correction to Energy=

Thermal correction to Enthalpy=
0.728437 (Hartree/Particle)

0.767631

0.768575 
Thermal correction to Gibbs Free Energy=

Sum of electronic and zero-point Energies= Sum of electronic and thermal Energies= Sum of electronic and thermal Enthalpies= Sum of electronic and thermal Free Energies=
0.655048

$-1861.839023$

$-1861.799829$

$-1861.798885$

$-1861.912412$

\begin{tabular}{lrrr}
$\mathrm{C}$ & 0.66177600 & -0.89363000 & -0.49210000 \\
$\mathrm{~S}$ & 0.30062200 & -0.16337400 & -1.99868500 \\
$\mathrm{~N}$ & -0.42765900 & -1.01883400 & 0.42152600 \\
$\mathrm{H}$ & -0.32288700 & -1.78004800 & 1.07708400 \\
$\mathrm{~N}$ & 1.74554800 & -1.37353700 & 0.03971100 \\
$\mathrm{C}$ & 2.96562100 & -1.66512400 & -0.59039100 \\
$\mathrm{C}$ & 3.95913400 & -2.14856100 & 0.27453400 \\
$\mathrm{C}$ & 3.28543500 & -1.52226800 & -1.94805000 \\
$\mathrm{C}$ & 5.23312900 & -2.43798700 & -0.17894400 \\
$\mathrm{H}$ & 3.68943300 & -2.30247100 & 1.31343000 \\
$\mathrm{C}$ & 4.56929400 & -1.81953100 & -2.39726400 \\
$\mathrm{H}$ & 2.52971400 & -1.19772700 & -2.64880900 \\
$\mathrm{C}$ & 5.55152700 & -2.26086600 & -1.52271800 \\
$\mathrm{H}$ & 6.54650400 & -2.48886700 & -1.88652300 \\
$\mathrm{H}$ & 2.60554700 & 0.11765500 & 1.74368200 \\
$\mathrm{C}$ & 3.62220200 & 1.66694300 & 0.68841000 \\
$\mathrm{C}$ & 2.95822900 & 1.13643600 & 1.93783900 \\
$\mathrm{C}$ & 3.99963800 & 1.13983200 & 3.06438000 \\
$\mathrm{C}$ & 5.25478600 & 0.38878900 & 2.61952500 \\
$\mathrm{C}$ & 5.77816600 & 0.96341800 & 1.31422400 \\
$\mathrm{H}$ & 4.25808400 & 2.17285600 & 3.32659200 \\
$\mathrm{H}$ & 3.56897600 & 0.68565700 & 3.95781600 \\
$\mathrm{H}$ & 6.03757600 & 0.45934000 & 3.37847100 \\
$\mathrm{H}$ & 5.02516700 & -0.66954200 & 2.47440900 \\
$\mathrm{H}$ & 6.16223700 & 1.98263500 & 1.46047500 \\
$\mathrm{H}$ & 6.56672800 & 0.35150400 & 0.88148600 \\
$\mathrm{O}$ & 4.75410900 & 0.99603800 & 0.31083300 \\
$\mathrm{H}$ & 3.78066100 & 2.75444800 & 0.72998400 \\
$\mathrm{H}$ & 2.09633000 & 1.76031400 & 2.18602500 \\
$\mathrm{C}$ & 3.19099500 & 1.89266100 & -1.77377000 \\
$\mathrm{H}$ & 4.05450900 & 1.27436400 & -2.00287400 \\
$\mathrm{H}$ & 3.43683800 & 2.94915700 & -1.69920200 \\
$\mathrm{H}$ & 2.35954600 & 1.70620500 & -2.44696300 \\
$\mathrm{O}$ & 2.68591200 & 1.51027900 & -0.44336700 \\
$\mathrm{H}$ & 2.27816500 & 0.60677300 & -0.46090100 \\
$\mathrm{H}$ & 5.97814500 & -2.81619000 & 0.51283100 \\
$\mathrm{H}$ & 4.79454600 & -1.71118600 & -3.45271400 \\
$\mathrm{C}$ & -1.65241800 & -0.48633600 & 0.24900300 \\
$\mathrm{C}$ & -2.47235700 & 0.59315500 & 0.01419600 \\
$\mathrm{C}$ & -3.01200200 & -0.67472300 & 0.28663300 \\
$\mathrm{~N}$ & -2.68643900 & 1.86376800 & -0.26078000 \\
& & & \\
\hline
\end{tabular}

$\begin{array}{lrrr}\mathrm{N} & -4.02783900 & -1.49584900 & 0.44942200 \\ \mathrm{C} & -4.08345700 & 2.29619400 & -0.49595800 \\ \mathrm{C} & -1.63497700 & 2.88885300 & -0.04460100 \\ \mathrm{C} & -5.41312000 & -0.98870300 & 0.50389400 \\ \mathrm{C} & -3.76419900 & -2.95415500 & 0.46050900 \\ \mathrm{C} & -4.72436300 & 2.80371300 & 0.79502300 \\ \mathrm{H} & -4.60655300 & 1.39487100 & -0.82205100 \\ \mathrm{C} & -4.19342900 & 3.30516600 & -1.63294200 \\ \mathrm{C} & -0.87058800 & 3.22804000 & -1.32105900 \\ \mathrm{H} & -2.18041200 & 3.77785400 & 0.28420400 \\ \mathrm{C} & -0.69078200 & 2.48959200 & 1.08598700 \\ \mathrm{H} & -5.31802300 & 0.09128400 & 0.62339800 \\ \mathrm{C} & -6.15258600 & -1.27551400 & -0.79961400 \\ \mathrm{C} & -6.15112800 & -1.51363100 & 1.73220100 \\ \mathrm{H} & -4.74810700 & -3.42283100 & 0.40600500 \\ \mathrm{C} & -2.97348000 & -3.38285300 & -0.77243200 \\ \mathrm{C} & -3.10563400 & -3.37954700 & 1.76928100 \\ \mathrm{H} & -4.64264600 & 2.06380400 & 1.59593700 \\ \mathrm{H} & -4.25288500 & 3.72793600 & 1.13855800 \\ \mathrm{H} & -5.78328900 & 3.01569900 & 0.63101000 \\ \mathrm{H} & -3.71291000 & 4.25513100 & -1.38721100 \\ \mathrm{H} & -3.75101200 & 2.91242400 & -2.54974900 \\ \mathrm{H} & -5.24863900 & 3.51167700 & -1.82277400 \\ \mathrm{H} & -0.32738900 & 2.34889200 & -1.67075500 \\ \mathrm{H} & -1.53684400 & 3.56273900 & -2.11571100 \\ \mathrm{H} & -0.16026300 & 4.03346500 & -1.11365200 \\ \mathrm{H} & -1.23879100 & 2.22449800 & 1.99355600 \\ \mathrm{H} & -0.05739000 & 1.64725900 & 0.79823700 \\ \mathrm{H} & -0.03936500 & 3.33499400 & 1.31878300 \\ \mathrm{H} & -6.27002900 & -2.35008300 & -0.96317100 \\ \mathrm{H} & -7.15144700 & -0.83485900 & -0.76966300 \\ \mathrm{H} & -5.61593700 & -0.85747200 & -1.65485000 \\ \mathrm{H} & -5.60085700 & -1.28599700 & 2.64751400 \\ \mathrm{H} & -7.13222700 & -1.03890700 & 1.79666300 \\ \mathrm{H} & -6.31432400 & -2.59301500 & 1.68335900 \\ \mathrm{H} & -2.87857600 & -4.47036400 & -0.78952500 \\ \mathrm{H} & -3.47889400 & -3.06812100 & -1.68761900 \\ \mathrm{H} & -1.96476600 & -2.96181100 & -0.77930100 \\ \mathrm{H} & -2.12170400 & -2.91598600 & 1.88519900 \\ \mathrm{H} & -3.71719400 & -3.09768600 & 2.62832700 \\ & -2.96321300 & -4.46183800 & 1.78628000\end{array}$




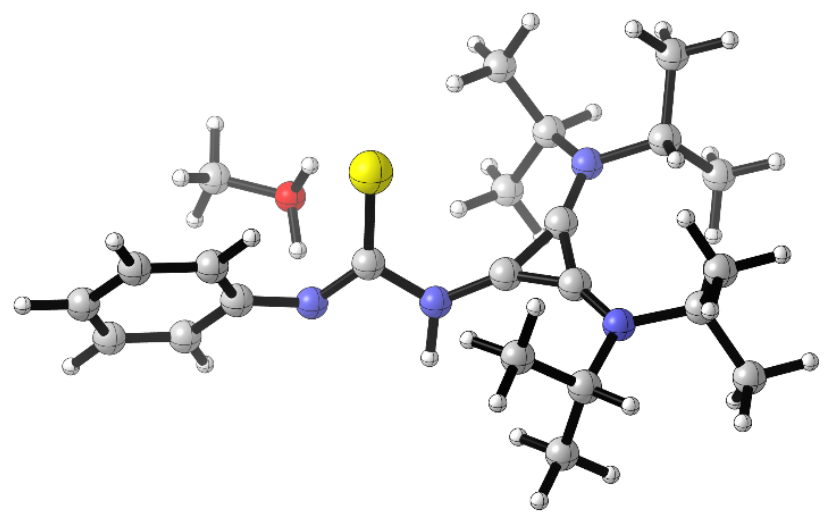

Figure S37. Optimized Structure of Z,Z-1-An-TS3-MA.

- Thermochemistry -

$(1$ imaginary frequency $=-562.58 \mathrm{~Hz})$

Zero-point correction $=$

Thermal correction to Energy=

0.597985 (Hartree/Particle)

Thermal correction to Enthalpy=

0.631601

Thermal correction to Gibbs Free Energy=

0.632545

Sum of electronic and zero-point Energies=

0.531255

Sum of electronic and thermal Energies=

$-1591.409820$

Sum of electronic and thermal Enthalpies=

$-1591.376205$

Sum of electronic and thermal Free Energies=

$-1591.375261$

$-1591.476551$

$\begin{array}{lrrr}\mathrm{C} & -1.64224600 & -0.16899400 & -0.07054400 \\ \mathrm{~S} & -1.46044300 & 0.73166400 & 1.36335300 \\ \mathrm{~N} & -0.49843400 & -0.58624500 & -0.74110800 \\ \mathrm{H} & -0.66571600 & -1.28202500 & -1.45566700 \\ \mathrm{~N} & -2.73785500 & -0.45083000 & -0.76949700 \\ \mathrm{C} & -3.99536200 & -0.77312200 & -0.20057700 \\ \mathrm{C} & -5.06178000 & -0.81871800 & -1.10740300 \\ \mathrm{C} & -4.24275900 & -1.07127500 & 1.14192700 \\ \mathrm{C} & -6.34690900 & -1.11695800 & -0.68225300 \\ \mathrm{H} & -4.85545000 & -0.63527700 & -2.15679800 \\ \mathrm{C} & -5.53280900 & -1.38187100 & 1.55667000 \\ \mathrm{H} & -3.43935300 & -1.05876900 & 1.86506700 \\ \mathrm{C} & -6.59167800 & -1.39498200 & 0.65779100 \\ \mathrm{H} & -7.59326800 & -1.63331200 & 0.99471200 \\ \mathrm{O} & -3.23948000 & 2.03357700 & -0.73900500 \\ \mathrm{H} & -2.76758000 & 2.00529100 & 0.14079000 \\ \mathrm{H} & -7.15600300 & -1.14700100 & -1.40300700 \\ \mathrm{H} & -5.70805400 & -1.61271500 & 2.60130300 \\ \mathrm{C} & 0.78644900 & -0.28863400 & -0.40516300 \\ \mathrm{C} & 1.78828500 & 0.61375000 & -0.14052300 \\ \mathrm{C} & 2.04658000 & -0.77757000 & -0.18295300 \\ \mathrm{~N} & 2.25726300 & 1.82576000 & 0.03216100 \\ \mathrm{~N} & 2.85193400 & -1.80848300 & -0.09556800 \\ \mathrm{C} & 3.68135900 & 1.97275100 & 0.42539500 \\ \mathrm{C} & 1.49925700 & 3.02164600 & -0.42676100 \\ \mathrm{C} & 4.31248200 & -1.63298500 & 0.06066200\end{array}$

$\begin{array}{lrrr}\mathrm{C} & 2.26307200 & -3.17082800 & -0.08310400 \\ \mathrm{C} & 4.56861500 & 2.11309100 & -0.80941300 \\ \mathrm{H} & 3.92778500 & 1.04277100 & 0.94187400 \\ \mathrm{C} & 3.89527500 & 3.09982600 & 1.42803300 \\ \mathrm{C} & 0.70158900 & 3.67102200 & 0.69946000 \\ \mathrm{H} & 2.27117100 & 3.72049400 & -0.75957400 \\ \mathrm{C} & 0.62474800 & 2.69983400 & -1.63406100 \\ \mathrm{H} & 4.49466100 & -0.57258800 & -0.11426400 \\ \mathrm{C} & 4.74928800 & -1.97953900 & 1.48049600 \\ \mathrm{C} & 5.08095000 & -2.40527000 & -1.00752000 \\ \mathrm{H} & 3.09056900 & -3.83482500 & 0.17051700 \\ \mathrm{C} & 1.20496200 & -3.30741700 & 1.00752500 \\ \mathrm{C} & 1.74783200 & -3.55614500 & -1.46602600 \\ \mathrm{H} & 4.40889900 & 1.28980100 & -1.51120600 \\ \mathrm{H} & 4.37002300 & 3.04972100 & -1.33667900 \\ \mathrm{H} & 5.62137500 & 2.11665400 & -0.51844900 \\ \mathrm{H} & 3.68792300 & 4.08120400 & 0.99572100 \\ \mathrm{H} & 3.27517400 & 2.96395000 & 2.31519200 \\ \mathrm{H} & 4.94135200 & 3.09649400 & 1.74062200 \\ \mathrm{H} & -0.05553900 & 2.97884200 & 1.07012600 \\ \mathrm{H} & 1.33766600 & 3.96654500 & 1.53314600 \\ \mathrm{H} & 0.20622400 & 4.56799600 & 0.31943800 \\ \mathrm{H} & 1.19442900 & 2.20154400 & -2.42224000 \\ \mathrm{H} & -0.22700100 & 2.07291900 & -1.36138500 \\ \mathrm{H} & 0.22728500 & 3.63025300 & -2.04308100 \\ \mathrm{H} & 4.57950000 & -3.03613100 & 1.70364900\end{array}$




$\begin{array}{rrrrrrrr}\mathrm{H} & 5.81671700 & -1.78497700 & 1.60294600 & \mathrm{H} & 0.92864100 & -2.90250400 & -1.77900600 \\ \mathrm{H} & 4.20257200 & -1.38269100 & 2.21451500 & \mathrm{H} & 2.53814900 & -3.49531000 & -2.21600200 \\ \mathrm{H} & 4.75521900 & -2.12028600 & -2.01009100 & \mathrm{H} & 1.36341500 & -4.57772300 & -1.45173900 \\ \mathrm{H} & 6.14600700 & -2.18317200 & -0.91766800 & \mathrm{H} & -3.08605800 & 0.98753900 & -1.00786700 \\ \mathrm{H} & 4.96393700 & -3.48589300 & -0.89703300 & \mathrm{C} & -4.63356200 & 2.42513400 & -0.56779400 \\ \mathrm{H} & 0.88726900 & -4.34924900 & 1.08041500 & \mathrm{H} & -5.09586600 & 1.80951400 & 0.20243600 \\ \mathrm{H} & 1.60311600 & -3.00233600 & 1.97730100 & \mathrm{H} & -4.63612600 & 3.47999300 & -0.30243900 \\ \mathrm{H} & 0.31694000 & -2.70677300 & 0.79399800 & \mathrm{H} & -5.11925500 & 2.26888900 & -1.52627300\end{array}$

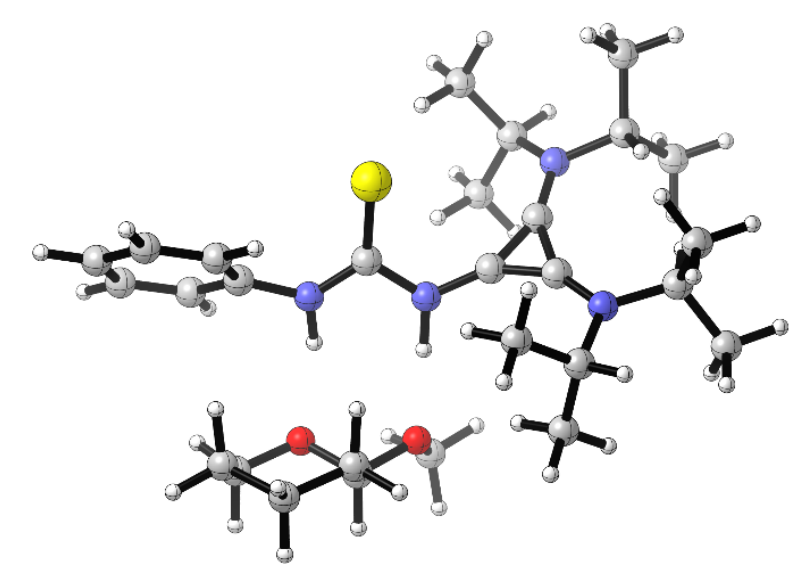

Figure S38. Optimized Structure of Z,Z-1-An-INT 5.

- Thermochemistry -

(0 imaginary frequencies)

Zero-point correction $=$

Thermal correction to Energy=

Thermal correction to Enthalpy=

Thermal correction to Gibbs Free Energy=

Sum of electronic and zero-point Energies=

Sum of electronic and thermal Energies=

Sum of electronic and thermal Enthalpies=

Sum of electronic and thermal Free Energies=
0.730296 (Hartree/Particle)

0.770089

0.771033

0.655788

$-1861.918663$

$-1861.878869$

$-1861.877925$

$-1861.993170$

$\begin{array}{lrrrrrrr}\mathrm{C} & 0.92697800 & 1.01534800 & -0.30996400 & \mathrm{C} & 2.63356700 & -2.67607300 & -0.19378400 \\ \mathrm{~S} & 0.32051200 & 2.07127500 & -1.44036700 & \mathrm{C} & 4.08865600 & -2.86698700 & -0.63324900 \\ \mathrm{~N} & 0.10244100 & 0.09301300 & 0.33252700 & \mathrm{C} & 4.94875000 & -1.68910000 & -0.17078300 \\ \mathrm{H} & 0.51758300 & -0.74223500 & 0.75874800 & \mathrm{C} & 4.75467900 & -1.44374000 & 1.31773500 \\ \mathrm{~N} & 2.20392900 & 0.89664200 & 0.09075000 & \mathrm{H} & 4.47694600 & -3.79665300 & -0.20046600 \\ \mathrm{C} & 3.35270100 & 1.56036700 & -0.41917200 & \mathrm{H} & 4.14093600 & -2.98012800 & -1.71758800 \\ \mathrm{C} & 3.60553700 & 1.66936200 & -1.78257800 & \mathrm{H} & 6.00650000 & -1.88300100 & -0.36540300 \\ \mathrm{C} & 4.28834800 & 2.01182900 & 0.50667000 & \mathrm{H} & 4.67906700 & -0.78280700 & -0.72146200 \\ \mathrm{C} & 4.79598800 & 2.24057700 & -2.21027700 & \mathrm{H} & 5.14837500 & -2.28242600 & 1.90821500 \\ \mathrm{H} & 2.87772800 & 1.31606100 & -2.50001000 & \mathrm{H} & 5.24678600 & -0.52941400 & 1.64616800 \\ \mathrm{C} & 5.48264400 & 2.56866600 & 0.06945900 & \mathrm{O} & 3.36752600 & -1.27234400 & 1.63608900 \\ \mathrm{H} & 4.07493800 & 1.92557500 & 1.56671100 & \mathrm{H} & 2.95810200 & -3.27021400 & 1.86747200 \\ \mathrm{C} & 5.73774700 & 2.68735400 & -1.29068400 & \mathrm{H} & 2.02916700 & -3.55854300 & -0.41701600 \\ \mathrm{H} & 6.66503300 & 3.12975700 & -1.63416600 & \mathrm{C} & 1.07260800 & -2.05737200 & 3.09094400 \\ \mathrm{H} & 2.19132500 & -1.82004700 & -0.71610500 & \mathrm{H} & 1.69276600 & -1.27984400 & 3.54053400 \\ \mathrm{C} & 2.58655900 & -2.40214400 & 1.29710000 & \mathrm{H} & 1.30504500 & -3.02524400 & 3.54520000\end{array}$




$\begin{array}{lrrrrrrr}\mathrm{H} & 0.01862200 & -1.83139000 & 3.24691000 & \mathrm{C} & -1.30779300 & -3.54965800 & -0.14825500 \\ \mathrm{O} & 1.27444500 & -2.11599100 & 1.68050000 & \mathrm{H} & -5.08405300 & 0.24882300 & 1.79557000 \\ \mathrm{H} & 2.42455800 & 0.23202100 & 0.83182000 & \mathrm{H} & -5.44389500 & 1.92987500 & 2.21464000 \\ \mathrm{H} & 4.98902700 & 2.33302900 & -3.27244000 & \mathrm{H} & -6.51953900 & 1.04828900 & 1.13175300 \\ \mathrm{H} & 6.20812000 & 2.91953500 & 0.79379300 & \mathrm{H} & -5.17271600 & 3.78596300 & 0.35255600 \\ \mathrm{C} & -1.24594400 & 0.08883900 & 0.14926400 & \mathrm{H} & -4.62922300 & 3.27733500 & -1.25969100 \\ \mathrm{C} & -2.46423400 & 0.71575400 & 0.23890300 & \mathrm{H} & -6.23960000 & 2.83507200 & -0.67587000 \\ \mathrm{C} & -2.35506000 & -0.61659000 & -0.23064400 & \mathrm{H} & -1.23571200 & 3.66223000 & 0.02099600 \\ \mathrm{~N} & -3.22555100 & 1.74244900 & 0.52297800 & \mathrm{H} & -2.84126600 & 4.37806400 & -0.18707000 \\ \mathrm{~N} & -2.88988600 & -1.71819900 & -0.70159400 & \mathrm{H} & -1.85680400 & 4.81908700 & 1.21226000 \\ \mathrm{C} & -4.66994800 & 1.66706300 & 0.19684400 & \mathrm{H} & -2.16020500 & 1.50832800 & 2.98288200 \\ \mathrm{C} & -2.72226700 & 2.85874000 & 1.37061600 & \mathrm{H} & -0.79126600 & 2.05628800 & 1.98415900 \\ \mathrm{C} & -4.35840800 & -1.87916800 & -0.76437400 & \mathrm{H} & -1.53882100 & 3.15680600 & 3.13576200 \\ \mathrm{C} & -2.00137200 & -2.76767400 & -1.25937200 & \mathrm{H} & -4.47222200 & -2.48483100 & -2.85204100 \\ \mathrm{C} & -5.47269700 & 1.19428600 & 1.40684100 & \mathrm{H} & -5.95267100 & -1.76716600 & -2.21863600 \\ \mathrm{H} & -4.74312200 & 0.91948400 & -0.59546600 & \mathrm{H} & -4.56228300 & -0.73543700 & -2.59966200 \\ \mathrm{C} & -5.19833500 & 2.97546800 & -0.37915200 & \mathrm{H} & -4.43913300 & -3.28354100 & 0.89150800 \\ \mathrm{C} & -2.13108000 & 3.99700700 & 0.54673600 & \mathrm{H} & -5.89618500 & -3.23059900 & -0.10960200 \\ \mathrm{H} & -3.61143500 & 3.22323100 & 1.89082700 & \mathrm{H} & -4.46305300 & -4.06239300 & -0.70184400 \\ \mathrm{C} & -1.74501700 & 2.35303500 & 2.42699500 & \mathrm{H} & -0.47083900 & -2.99623900 & -2.75331600 \\ \mathrm{H} & -4.76124300 & -1.07478700 & -0.14738300 & \mathrm{H} & -1.53706600 & -1.61267900 & -3.04113700 \\ \mathrm{C} & -4.86271200 & -1.70441700 & -2.19343900 & \mathrm{H} & -0.28451200 & -1.52890800 & -1.79232600 \\ \mathrm{C} & -4.80531300 & -3.19436900 & -0.13330100 & \mathrm{H} & -0.60740800 & -2.92216900 & 0.40877500 \\ \mathrm{H} & -2.66828200 & -3.44328300 & -1.79738100 & \mathrm{H} & -2.03348300 & -3.96904400 & 0.55105200 \\ \mathrm{C} & -1.01763700 & -2.18431600 & -2.26945500 & \mathrm{H} & -0.73672400 & -4.37540600 & -0.57943700\end{array}$

iv. Z,E Conformation

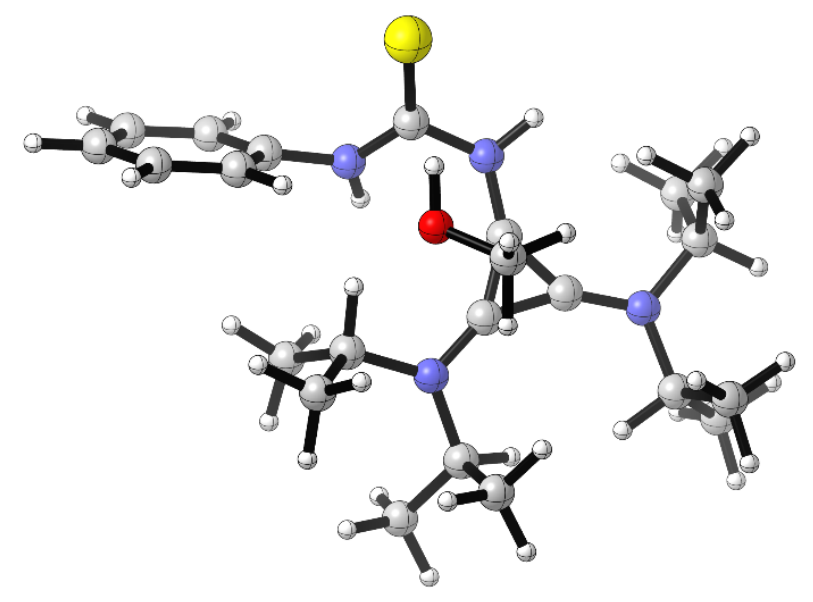

Figure S39. Optimized Structure of Z,E-1-An-INT 1 .

- Thermochemistry -

(0 imaginary frequencies)

Zero-point correction $=\quad 0.600527($ Hartree/Particle $)$ 
Thermal correction to Energy=

Thermal correction to Enthalpy=

Thermal correction to Gibbs Free Energy=

Sum of electronic and zero-point Energies=

Sum of electronic and thermal Energies=

Sum of electronic and thermal Enthalpies=

Sum of electronic and thermal Free Energies=
0.635884

0.636828

0.530948

$-1591.455251$

$-1591.419894$

$-1591.418950$

$-1591.524830$

$\begin{array}{lrrr}\mathrm{C} & 1.49672700 & -0.93537700 & -0.10908500 \\ \mathrm{~S} & 1.42645500 & -0.17576200 & 1.36234400 \\ \mathrm{~N} & 2.60021800 & -1.37874300 & -0.73537800 \\ \mathrm{H} & 2.52716000 & -1.52649700 & -1.73200000 \\ \mathrm{~N} & 0.33958900 & -1.21317900 & -0.85179700 \\ \mathrm{C} & 3.93131300 & -1.31024400 & -0.21900100 \\ \mathrm{C} & 4.28078600 & -2.02500600 & 0.91909800 \\ \mathrm{C} & 4.87132300 & -0.55069300 & -0.90390600 \\ \mathrm{C} & 5.58594200 & -1.96013100 & 1.38464300 \\ \mathrm{H} & 3.53610300 & -2.61654300 & 1.43692200 \\ \mathrm{C} & 6.17960300 & -0.50450500 & -0.43890500 \\ \mathrm{H} & 4.56969400 & 0.01218600 & -1.77952600 \\ \mathrm{C} & 6.53602500 & -1.20376200 & 0.70724000 \\ \mathrm{H} & 7.55502900 & -1.16188700 & 1.07270600 \\ \mathrm{H} & 0.31673400 & -2.07166700 & -1.38383200 \\ \mathrm{C} & 3.76920700 & 2.87153800 & -1.02249300 \\ \mathrm{H} & 3.63913600 & 3.67067400 & -0.28300900 \\ \mathrm{H} & 3.69156400 & 3.30807400 & -2.01855800 \\ \mathrm{H} & 4.77218400 & 2.44521900 & -0.90388800 \\ \mathrm{O} & 2.76249600 & 1.88049700 & -0.91693900 \\ \mathrm{H} & 2.81209100 & 1.48523800 & -0.04017800 \\ \mathrm{C} & -0.85046900 & -0.61571000 & -0.58067900 \\ \mathrm{C} & -1.54442300 & 0.53007100 & -0.28471700 \\ \mathrm{C} & -2.19901700 & -0.72036400 & -0.36868300 \\ \mathrm{~N} & -1.56307600 & 1.81661200 & -0.04918200 \\ \mathrm{~N} & -3.27186700 & -1.47276500 & -0.28560700 \\ \mathrm{C} & -2.75847000 & 2.37628700 & 0.63162000 \\ \mathrm{C} & -0.31935500 & 2.57414800 & -0.37471700 \\ \mathrm{C} & -4.61177300 & -0.86402500 & -0.13556900 \\ \mathrm{C} & -3.10012900 & -2.94302900 & -0.20666000 \\ \mathrm{C} & -2.59733700 & 2.35078700 & 2.15167400 \\ \mathrm{H} & -3.56234300 & 1.68171100 & 0.38001600 \\ \mathrm{C} & -3.17557300 & 3.74291800 & 0.10540900 \\ \mathrm{C} & 0.23648700 & 3.35820800 & 0.80492400 \\ & & & \end{array}$

$\begin{array}{lrrr}\mathrm{H} & -4.47989400 & 0.18255800 & -0.41689300 \\ \mathrm{C} & -5.61051500 & -1.46758100 & -1.11813500 \\ \mathrm{C} & -5.07892800 & -0.93201200 & 1.31457600 \\ \mathrm{H} & -4.09010300 & -3.33508800 & 0.02904200 \\ \mathrm{C} & -2.67160200 & -3.51397900 & -1.55461100 \\ \mathrm{C} & -2.16318500 & -3.32823500 & 0.93466600 \\ \mathrm{H} & -2.26133500 & 1.36817100 & 2.49264000 \\ \mathrm{H} & -1.87859300 & 3.09452700 & 2.49641000 \\ \mathrm{H} & -3.55866200 & 2.56820200 & 2.62333600 \\ \mathrm{H} & -2.45587700 & 4.52125300 & 0.36201600 \\ \mathrm{H} & -3.31269200 & 3.73160100 & -0.97686600 \\ \mathrm{H} & -4.12702100 & 4.01468600 & 0.56693100 \\ \mathrm{H} & -0.39064700 & 4.20816500 & 1.08178700 \\ \mathrm{H} & 0.37349800 & 2.71444900 & 1.67504600 \\ \mathrm{H} & 1.21395100 & 3.74854000 & 0.51569400 \\ \mathrm{H} & -5.83200500 & -2.51380800 & -0.89495500 \\ \mathrm{H} & -6.55171600 & -0.91813100 & -1.05704500 \\ \mathrm{H} & -5.24151500 & -1.39873200 & -2.14339700 \\ \mathrm{H} & -5.20096600 & -1.96756500 & 1.64403500 \\ \mathrm{H} & -4.36251000 & -0.44211900 & 1.97883500 \\ \mathrm{H} & -6.04511100 & -0.43491800 & 1.42343300 \\ \mathrm{H} & -2.58088200 & -4.60003800 & -1.49275200 \\ \mathrm{H} & -3.39598300 & -3.27039100 & -2.33340100 \\ \mathrm{H} & -1.69905800 & -3.11752400 & -1.86284900 \\ \mathrm{H} & -1.13828400 & -2.99161000 & 0.75474300 \\ \mathrm{H} & -2.50127300 & -2.89560100 & 1.87830600 \\ \mathrm{H} & -2.14058900 & -4.41413100 & 1.04419800 \\ \mathrm{H} & 5.86354800 & -2.50916800 & 2.27623500 \\ \mathrm{H} & 6.91824300 & 0.08145300 & -0.97283000 \\ \mathrm{H} & 0.41581600 & 1.80540200 & -0.60918200 \\ \mathrm{C} & -0.47169700 & 3.42665100 & -1.63232400 \\ \mathrm{H} & -1.04064300 & 4.34056700 & -1.45856300 \\ \mathrm{H} & 0.52702100 & 3.70961800 & -1.97152800 \\ \mathrm{H} & -0.95100400 & 2.86212300 & -2.43552000 \\ & & \end{array}$




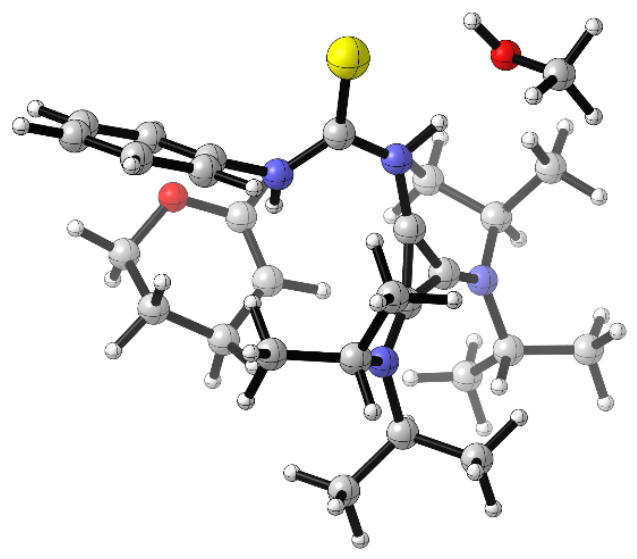

Figure S40. Optimized Structure of $Z, E-1-A n-I_{N T}$.

- Thermochemistry -

(0 imaginary frequencies)

Zero-point correction=

Thermal correction to Energy=

0.728381 (Hartree/Particle)

Thermal correction to Enthalpy=

0.769422

Thermal correction to Gibbs Free Energy=

0.770366

Sum of electronic and zero-point Energies=

0.653712

Sum of electronic and thermal Energies=

$-1861.890023$

Sum of electronic and thermal Enthalpies=

$-1861.848982$

Sum of electronic and thermal Free Energies=

$-1861.848038$

$-1861.964692$

$\begin{array}{lrrr}\mathrm{C} & -1.28241700 & 1.89310400 & 0.09339200 \\ \mathrm{~S} & -2.02861600 & 3.33359900 & 0.42915200 \\ \mathrm{~N} & 0.10132800 & 1.83181900 & -0.06865800 \\ \mathrm{H} & 0.56809300 & 2.74757500 & -0.17881700 \\ \mathrm{~N} & -1.89462900 & 0.70249300 & -0.07819300 \\ \mathrm{C} & -3.30378500 & 0.50016500 & 0.05138600 \\ \mathrm{C} & -3.90574100 & 0.59883100 & 1.29997400 \\ \mathrm{C} & -4.04985600 & 0.17328500 & -1.07327100 \\ \mathrm{C} & -5.26754200 & 0.36835900 & 1.42162200 \\ \mathrm{H} & -3.31135400 & 0.86860400 & 2.16455700 \\ \mathrm{C} & -5.41126700 & -0.07150800 & -0.93982900 \\ \mathrm{H} & -3.56481500 & 0.10867700 & -2.04037900 \\ \mathrm{C} & -6.02125600 & 0.02761800 & 0.30364900 \\ \mathrm{H} & -7.08470800 & -0.15358700 & 0.40327900 \\ \mathrm{H} & -1.38732400 & 0.00665800 & -0.61852500 \\ \mathrm{C} & -0.74700500 & -1.02670900 & -2.85292800 \\ \mathrm{C} & -0.04220100 & -1.75436600 & -1.98163500 \\ \mathrm{C} & -0.63557700 & -2.93252500 & -1.26079700 \\ \mathrm{C} & -2.15893400 & -2.91425200 & -1.40489000 \\ \mathrm{C} & -2.54425700 & -2.54045700 & -2.82763600 \\ \mathrm{H} & -0.22287000 & -3.86755700 & -1.65687200 \\ \mathrm{H} & -0.36161800 & -2.89443800 & -0.20278800 \\ \mathrm{H} & -2.59267100 & -3.88638700 & -1.16118500 \\ \mathrm{H} & -2.60085300 & -2.18784600 & -0.71567200 \\ \mathrm{H} & -2.16363300 & -3.28200800 & -3.53892100 \\ \mathrm{H} & -3.62373300 & -2.46013200 & -2.94733200\end{array}$

$\begin{array}{lrrr}\mathrm{O} & -2.02510800 & -1.26008800 & -3.20935200 \\ \mathrm{H} & -0.34622900 & -0.15568500 & -3.35944300 \\ \mathrm{H} & 0.99744000 & -1.49300800 & -1.82563900 \\ \mathrm{C} & 1.50976500 & 4.76989400 & 1.20760900 \\ \mathrm{H} & 1.59524200 & 5.85279600 & 1.32439100 \\ \mathrm{H} & 2.50000900 & 4.32903500 & 1.32853900 \\ \mathrm{H} & 0.83787400 & 4.37351000 & 1.97639200 \\ \mathrm{O} & 1.05834000 & 4.42958500 & -0.09996400 \\ \mathrm{H} & 0.14964500 & 4.74168300 & -0.18982500 \\ \mathrm{H} & -5.74228700 & 0.45583200 & 2.39145000 \\ \mathrm{H} & -5.99734100 & -0.32457400 & -1.81545900 \\ \mathrm{C} & 0.88831500 & 0.77213500 & 0.21534100 \\ \mathrm{C} & 1.19633900 & -0.38098000 & 0.90208000 \\ \mathrm{C} & 2.10642400 & 0.17993900 & -0.01126400 \\ \mathrm{~N} & 0.91535400 & -1.35060600 & 1.75120400 \\ \mathrm{~N} & 3.24934300 & 0.17232500 & -0.65908700 \\ \mathrm{C} & 1.91722200 & -2.43263100 & 1.91784100 \\ \mathrm{C} & -0.16539400 & -1.20432100 & 2.76233700 \\ \mathrm{C} & 4.29990600 & -0.81534900 & -0.33075500 \\ \mathrm{C} & 3.46783400 & 1.13339700 & -1.77001500 \\ \mathrm{C} & 2.93522600 & -2.06058400 & 2.99435100 \\ \mathrm{H} & 2.41941000 & -2.50545800 & 0.95048400 \\ \mathrm{C} & 1.27849400 & -3.79182000 & 2.18105200 \\ \mathrm{C} & -1.46703000 & -1.83831700 & 2.28504900 \\ \mathrm{H} & 0.19519400 & -1.76035600 & 3.63072000 \\ \mathrm{C} & -0.34660700 & 0.24377800 & 3.20421500\end{array}$




$\begin{array}{rrrrrrrr}\mathrm{H} & 3.97346200 & -1.28660100 & 0.59557300 & \mathrm{H} & 0.60422100 & 0.69192300 & 3.50228400 \\ \mathrm{C} & 4.39566900 & -1.88324200 & -1.41632500 & \mathrm{H} & -0.80013700 & 0.86022700 & 2.42666500 \\ \mathrm{C} & 5.63627600 & -0.13821200 & -0.04175000 & \mathrm{H} & -1.01528200 & 0.27003500 & 4.06653400 \\ \mathrm{H} & 4.38904400 & 0.79719400 & -2.24783300 & \mathrm{H} & 4.71251300 & -1.45523000 & -2.37087900 \\ \mathrm{C} & 2.35170200 & 1.04913000 & -2.80607700 & \mathrm{H} & 5.13056400 & -2.63989200 & -1.13424200 \\ \mathrm{C} & 3.68556200 & 2.55013000 & -1.24930300 & \mathrm{H} & 3.43222500 & -2.37755100 & -1.56685200 \\ \mathrm{H} & 3.39763400 & -1.09098200 & 2.79027200 & \mathrm{H} & 5.53483100 & 0.61117500 & 0.74593800 \\ \mathrm{H} & 2.46378600 & -2.00731300 & 3.97910900 & \mathrm{H} & 6.35581400 & -0.88843700 & 0.29192300 \\ \mathrm{H} & 3.72467100 & -2.81353100 & 3.04613700 & \mathrm{H} & 6.05556100 & 0.34373000 & -0.92782800 \\ \mathrm{H} & 0.75809700 & -3.82821400 & 3.14047900 & \mathrm{H} & 2.59038400 & 1.69778400 & -3.65098100 \\ \mathrm{H} & 0.57948700 & -4.06939500 & 1.39131300 & \mathrm{H} & 2.23791100 & 0.02886000 & -3.18042700 \\ \mathrm{H} & 2.06622200 & -4.54694700 & 2.21143500 & \mathrm{H} & 1.39564300 & 1.38517200 & -2.39572000 \\ \mathrm{H} & -1.83246100 & -1.33801700 & 1.38873100 & \mathrm{H} & 2.77073900 & 2.97686700 & -0.83182700 \\ \mathrm{H} & -1.34095700 & -2.89916000 & 2.06636100 & \mathrm{H} & 4.46908600 & 2.57189300 & -0.48977500 \\ \mathrm{H} & -2.23440400 & -1.74131000 & 3.05610100 & \mathrm{H} & 3.99092900 & 3.20164500 & -2.07077000\end{array}$

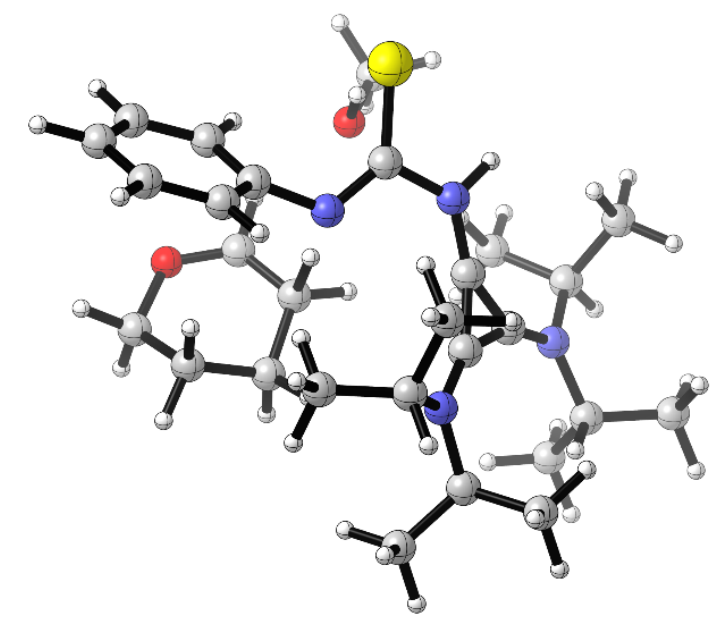

Figure S41. Optimized Structure of Z,E-1-An-TS . $_{\text {. }}$

- Thermochemistry -

$(1$ imaginary frequency $=-389.29 \mathrm{~Hz})$

Zero-point correction $=$

Thermal correction to Energy=

Thermal correction to Enthalpy=

Thermal correction to Gibbs Free Energy=

Sum of electronic and zero-point Energies=

Sum of electronic and thermal Energies=

Sum of electronic and thermal Enthalpies=

Sum of electronic and thermal Free Energies=
0.723295 (Hartree/Particle)

0.763740

0.764684

0.649390

$-1861.845928$

$-1861.805483$

$-1861.804539$

$-1861.919833$

$\begin{array}{lcccccccc}\mathrm{C} & 1.18795200 & 1.00065200 & -1.63964800 & \mathrm{C} & 3.55111200 & -1.42619800 & -1.32705900 \\ \mathrm{~S} & 2.00021400 & 2.13437700 & -2.59192600 & \mathrm{C} & 3.86904200 & 0.64174000 & -0.13918100 \\ \mathrm{~N} & -0.21464200 & 1.18415800 & -1.55557100 & \mathrm{C} & 4.88607800 & -1.74578300 & -1.11803800 \\ \mathrm{H} & -0.52388400 & 2.08407800 & -1.89106500 & \mathrm{H} & 2.91023300 & -2.08711500 & -1.89707000 \\ \mathrm{~N} & 1.63674700 & 0.01607800 & -0.90150500 & \mathrm{C} & 5.19911900 & 0.31105200 & 0.08251400 \\ \mathrm{C} & 3.02753600 & -0.23627200 & -0.82426600 & \mathrm{H} & 3.48374300 & 1.60111700 & 0.18643300\end{array}$




\begin{tabular}{|c|c|c|c|c|c|c|c|}
\hline $\mathrm{C}$ & 5.71048000 & -0.88783700 & -0.39854900 & $\mathrm{H}$ & -3.07890100 & -2.08045000 & 0.90416400 \\
\hline $\mathrm{H}$ & 6.75155000 & -1.14028600 & -0.23618400 & $\mathrm{C}$ & -2.21886000 & -4.00995500 & 1.02294500 \\
\hline $\mathrm{H}$ & 1.18779200 & 0.18021100 & 0.56652400 & $\mathrm{C}$ & 0.60406600 & -3.29845200 & -0.65999500 \\
\hline $\mathrm{C}$ & 1.96257300 & 0.95481400 & 2.29874000 & $\mathrm{H}$ & -1.24690700 & -3.88517000 & -1.56879700 \\
\hline $\mathrm{C}$ & 0.86337000 & 0.25689600 & 1.75507000 & $\mathrm{C}$ & -0.44085900 & -2.29550700 & -2.73067500 \\
\hline $\mathrm{C}$ & 0.66470400 & -1.16245100 & 2.25653300 & $\mathrm{H}$ & -4.47116400 & -0.72580000 & 0.25672500 \\
\hline $\mathrm{C}$ & 2.04495700 & -1.80743300 & 2.39059200 & $\mathrm{C}$ & -4.72003600 & 0.36760500 & 2.07922000 \\
\hline $\mathrm{C}$ & 2.92635300 & -0.98436600 & 3.30781500 & $\mathrm{C}$ & -5.85409500 & 0.82960800 & -0.15170600 \\
\hline $\mathrm{H}$ & 0.14735900 & -1.16507500 & 3.22177400 & $\mathrm{H}$ & -4.18841600 & 2.79013600 & 0.75489900 \\
\hline $\mathrm{H}$ & 0.04806600 & -1.72307800 & 1.55361700 & $\mathrm{C}$ & -2.07961300 & 2.92530400 & 0.99690000 \\
\hline $\mathrm{H}$ & 1.97836700 & -2.81479800 & 2.80737100 & $\mathrm{C}$ & -3.37692700 & 3.14263700 & -1.18512800 \\
\hline $\mathrm{H}$ & 2.51655500 & -1.88872800 & 1.40722500 & $\mathrm{H}$ & -4.16975100 & -2.34545000 & -1.34167100 \\
\hline $\mathrm{H}$ & 2.61146300 & -1.03818300 & 4.35145300 & $\mathrm{H}$ & -3.50462000 & -3.97798700 & -1.46840500 \\
\hline $\mathrm{H}$ & 3.97716900 & -1.25499200 & 3.23391100 & $\mathrm{H}$ & -4.68183200 & -3.60837800 & -0.20895600 \\
\hline $\mathrm{O}$ & 2.91426000 & 0.44492900 & 2.98255300 & $\mathrm{H}$ & -1.82292700 & -4.81356000 & 0.39823500 \\
\hline $\mathrm{H}$ & 2.08647000 & 2.01635600 & 2.09103300 & $\mathrm{H}$ & -1.44832700 & -3.70714700 & 1.73472200 \\
\hline $\mathrm{H}$ & -0.00765800 & 0.90723200 & 1.66590000 & $\mathrm{H}$ & -3.05980800 & -4.41847600 & 1.58658200 \\
\hline $\mathrm{C}$ & 1.26991300 & 4.67155400 & 0.59711700 & $\mathrm{H}$ & 1.15577100 & -2.38052200 & -0.45791700 \\
\hline $\mathrm{H}$ & 2.22112700 & 5.19558300 & 0.46268400 & $\mathrm{H}$ & 0.42340600 & -3.82494000 & 0.27855900 \\
\hline $\mathrm{H}$ & 0.90729200 & 4.86247700 & 1.60836900 & $\mathrm{H}$ & 1.22364500 & -3.93927800 & -1.29166100 \\
\hline $\mathrm{H}$ & 0.54192100 & 5.07335900 & -0.11664300 & $\mathrm{H}$ & -1.37577900 & -2.00287200 & -3.21443200 \\
\hline $\mathrm{O}$ & 1.41946100 & 3.26800300 & 0.45440500 & $\mathrm{H}$ & 0.20539200 & -1.42164700 & -2.63211100 \\
\hline $\mathrm{H}$ & 1.72211800 & 3.08749400 & -0.45098000 & $\mathrm{H}$ & 0.06833600 & -3.00419500 & -3.38644700 \\
\hline $\mathrm{H}$ & 5.28701200 & -2.66668900 & -1.52565200 & $\mathrm{H}$ & -4.84787800 & 1.39340200 & 2.43514700 \\
\hline $\mathrm{H}$ & 5.84168000 & 1.00236000 & 0.61555200 & $\mathrm{H}$ & -5.58709000 & -0.20642400 & 2.41262200 \\
\hline $\mathrm{C}$ & -1.12061200 & 0.38784400 & -0.97431500 & $\mathrm{H}$ & -3.82758300 & -0.05035200 & 2.55229100 \\
\hline $\mathrm{C}$ & -1.64738500 & -0.83480000 & -0.61815800 & $\mathrm{H}$ & -5.74558400 & 0.75194700 & -1.23537100 \\
\hline $\mathrm{C}$ & -2.35417700 & 0.34567500 & -0.36569400 & $\mathrm{H}$ & -6.71757100 & 0.23308500 & 0.14926900 \\
\hline $\mathrm{N}$ & -1.59924300 & -2.15165000 & -0.52310900 & $\mathrm{H}$ & -6.07140800 & 1.86996500 & 0.10234500 \\
\hline $\mathrm{N}$ & -3.39192500 & 1.01463400 & 0.09533700 & $\mathrm{H}$ & -2.12173900 & 4.00279200 & 1.16967000 \\
\hline $\mathrm{C}$ & -2.70522000 & -2.82466000 & 0.19635400 & $\mathrm{H}$ & -2.06641200 & 2.42927100 & 1.97112000 \\
\hline $\mathrm{C}$ & -0.69466700 & -2.96080900 & -1.38233200 & $\mathrm{H}$ & -1.13509100 & 2.71889900 & 0.48470500 \\
\hline $\mathrm{C}$ & -4.60618700 & 0.31310500 & 0.55842800 & $\mathrm{H}$ & -2.54553400 & 2.83369800 & -1.82465200 \\
\hline $\mathrm{C}$ & -3.30267400 & 2.49033200 & 0.19247100 & $\mathrm{H}$ & -4.30472100 & 2.88032100 & -1.69587800 \\
\hline $\mathrm{C}$ & -3.82973900 & -3.20888100 & -0.76343300 & $\mathrm{H}$ & -3.33285000 & 4.22929100 & -1.08906500 \\
\hline
\end{tabular}

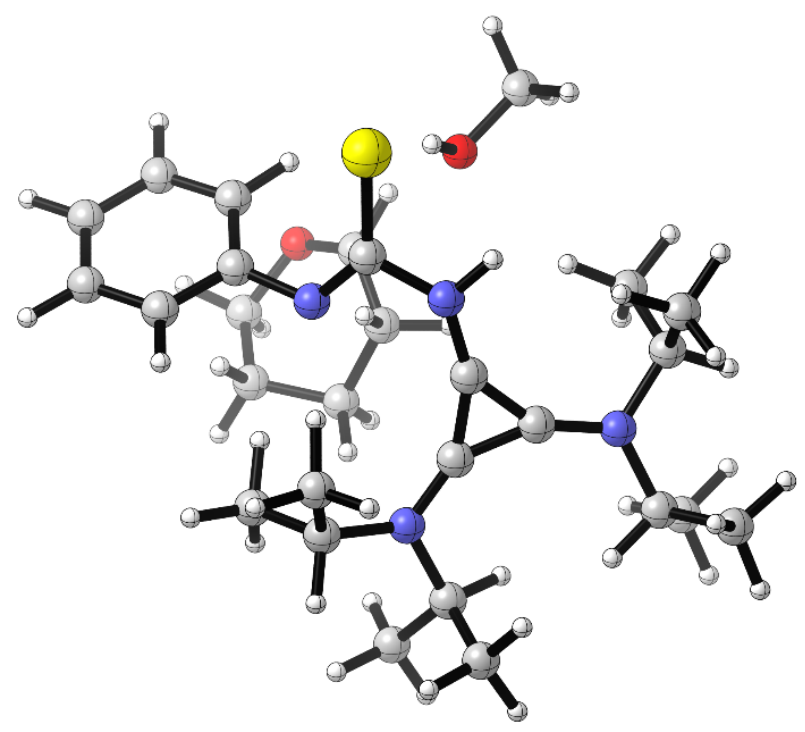

Figure S42. Optimized Structure of $Z, E-\mathbf{1}-A n-I_{N} \mathbf{T}_{3}$. 
- Thermochemistry -

(0 imaginary frequencies)

Zero-point correction=

Thermal correction to Energy=

Thermal correction to Enthalpy=

Thermal correction to Gibbs Free Energy=

Sum of electronic and zero-point Energies=

Sum of electronic and thermal Energies=

Sum of electronic and thermal Enthalpies=

Sum of electronic and thermal Free Energies=
0.725700 (Hartree/Particle)

0.766822

0.767766

0.650947

$-1861.845240$

$-1861.804118$

$-1861.803173$

$-1861.919993$

$\begin{array}{cccc}\mathrm{C} & 1.06913200 & 1.10209800 & -1.71055800 \\ \mathrm{~S} & 1.80107400 & 2.46664400 & -2.42117200 \\ \mathrm{~N} & -0.35041700 & 1.20067900 & -1.61946300 \\ \mathrm{H} & -0.69734200 & 2.11489300 & -1.86966700 \\ \mathrm{~N} & 1.55355100 & 0.01813900 & -1.19186800 \\ \mathrm{C} & 2.93510500 & -0.18867100 & -1.06031100 \\ \mathrm{C} & 3.49138900 & -1.38523400 & -1.52100300 \\ \mathrm{C} & 3.75064500 & 0.69374700 & -0.34329200 \\ \mathrm{C} & 4.81138900 & -1.70648000 & -1.23894100 \\ \mathrm{H} & 2.87703600 & -2.05305100 & -2.11205300 \\ \mathrm{C} & 5.06770100 & 0.36168500 & -0.04979900 \\ \mathrm{H} & 3.35857500 & 1.66185200 & -0.05389700 \\ \mathrm{C} & 5.60246300 & -0.84443800 & -0.48542500 \\ \mathrm{H} & 6.63441500 & -1.09535700 & -0.27075700 \\ \mathrm{H} & 1.12140100 & 0.22307700 & 0.68800800 \\ \mathrm{C} & 2.17877100 & 1.01020200 & 2.23317000 \\ \mathrm{C} & 1.04026000 & 0.21924600 & 1.81141400 \\ \mathrm{C} & 1.04564400 & -1.21277800 & 2.32596800 \\ \mathrm{C} & 2.47757300 & -1.74088300 & 2.23916500 \\ \mathrm{C} & 3.40498700 & -0.87570900 & 3.06269500 \\ \mathrm{H} & 0.68634900 & -1.25330200 & 3.35919300 \\ \mathrm{H} & 0.37329700 & -1.81782800 & 1.71801600 \\ \mathrm{H} & 2.55350700 & -2.76089400 & 2.62176500 \\ \mathrm{H} & 2.81513900 & -1.75296000 & 1.19921700 \\ \mathrm{H} & 3.25733000 & -0.98474900 & 4.13781600 \\ \mathrm{H} & 4.45455500 & -1.02013000 & 2.81702500 \\ \mathrm{O} & 3.20411000 & 0.56933300 & 2.81661900 \\ \mathrm{H} & 2.18413100 & 2.08299900 & 2.02581500 \\ \mathrm{H} & 0.13431700 & 0.80301900 & 2.00229900 \\ \mathrm{C} & 0.99220200 & 4.66340500 & 0.82286000 \\ \mathrm{H} & 1.87269100 & 5.26294000 & 0.57231300 \\ \mathrm{H} & 0.74645900 & 4.82647600 & 1.87349700 \\ \mathrm{H} & 0.14983900 & 5.00335200 & 0.21005600 \\ \mathrm{O} & 1.23598200 & 3.27704900 & 0.64484000 \\ \mathrm{H} & 1.47276300 & 3.13566300 & -0.29258500 \\ \mathrm{H} & 5.22906600 & -2.63293700 & -1.61652400 \\ \mathrm{H} & 5.68490900 & 1.06201400 & 0.50241400 \\ \mathrm{C} & -1.19346500 & 0.36591600 & -1.00385100 \\ \mathrm{C} & -1.64396800 & -0.87013600 & -0.58899700 \\ \mathrm{C} & -2.40756900 & 0.27601000 & -0.35843900 \\ \mathrm{~N} & -1.53134300 & -2.18149800 & -0.44237600\end{array}$

$\begin{array}{rrr}-3.46590200 & 0.90655800 & 0.11480600 \\ -2.59739000 & -2.86735900 & 0.32265500 \\ -0.63809900 & -2.99311700 & -1.31085900 \\ -4.64233200 & 0.16358600 & 0.60727500 \\ -3.43965100 & 2.38526200 & 0.18811500 \\ -3.73001300 & -3.32382600 & -0.59582300 \\ -2.98193500 & -2.11379400 & 1.01457100 \\ -2.05259900 & -4.00744700 & 1.17615800 \\ 0.69533600 & -3.26985500 & -0.62693500 \\ -1.16918500 & -3.93906500 & -1.44375600 \\ -0.45701200 & -2.36865400 & -2.68968400 \\ -4.46272900 & -0.87627900 & 0.33467300 \\ -4.75121700 & 0.25442800 & 2.12684200 \\ -5.91701000 & 0.60234000 & -0.10842400 \\ -4.33418800 & 2.65656100 & 0.75132000 \\ -2.23200000 & 2.87819800 & 0.98216400 \\ -3.55239400 & 3.01540800 & -1.19734700 \\ -4.10491900 & -2.49645400 & -1.20424200 \\ -3.39849600 & -4.11414000 & -1.27379400 \\ -4.55955900 & -3.72105200 & -0.00641700 \\ -1.64489000 & -4.81890500 & 0.56939400 \\ -1.27545800 & -3.65669800 & 1.85841700 \\ -2.86538500 & -4.42369400 & 1.77463200 \\ 1.21860600 & -2.32796900 & -0.46483400 \\ 0.56233000 & -3.78439800 & 0.32654300 \\ 1.31703000 & -3.90207500 & -1.26506900 \\ -1.42039100 & -2.13837000 & -3.15131100 \\ 0.15098400 & -1.46398100 & -2.63839800 \\ 0.06217700 & -3.07920700 & -3.33592800 \\ -4.91850100 & 1.28328900 & 2.45636500 \\ -5.59256900 & -0.34520400 & 2.48044700 \\ -3.84041300 & -0.11464400 & 2.60564200 \\ -5.80838400 & 0.50410500 & -1.19040600 \\ -6.74996900 & -0.02721000 & 0.21086000 \\ -6.18222500 & 1.63717700 & 0.12162600 \\ -2.30367800 & 3.95832600 & 1.12626400 \\ -2.20845000 & 2.40839000 & 1.96915400 \\ -1.27944900 & 2.68008300 & 0.48227300 \\ -2.71450700 & 2.73731400 & -1.84202800 \\ -4.47141200 & 2.70254100 & -1.69553200 \\ -3.55929800 & 4.10412800 & -1.11580100\end{array}$ 


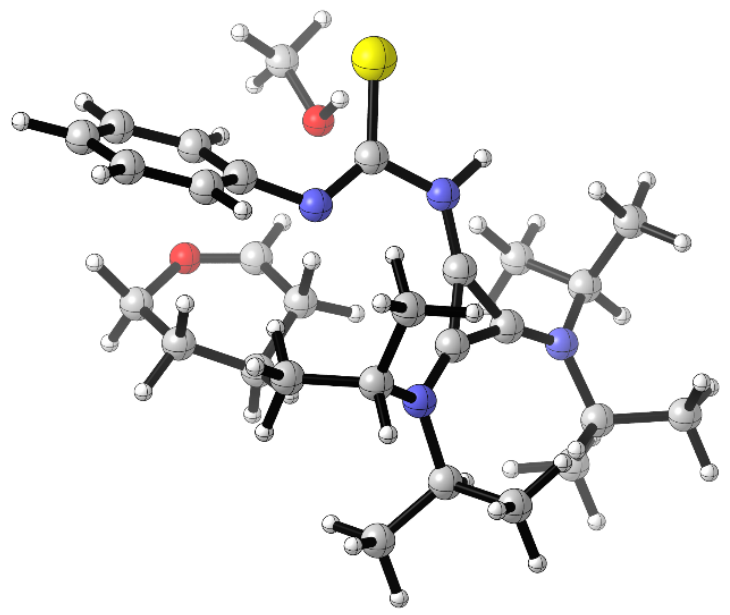

Figure S43. Optimized Structure of $Z, E-\mathbf{1}-A n-\mathbf{T S}_{2}$.

- Thermochemistry -

$(1$ imaginary frequency $=-39.39 \mathrm{~Hz})$

Zero-point correction=

Thermal correction to Energy=

0.726261 (Hartree/Particle)

Thermal correction to Enthalpy=

0.766376

0.767321

Thermal correction to Gibbs Free Energy=

Sum of electronic and zero-point Energies=

0.653395

Sum of electronic and thermal Energies=

$-1861.841574$

Sum of electronic and thermal Enthalpies=

$-1861.801458$

Sum of electronic and thermal Free Energies=

$-1861.800514$

$-1861.914440$

$\begin{array}{lrrr}\mathrm{C} & -1.13612200 & -0.87289100 & -1.67846500 \\ \mathrm{~S} & -1.99670000 & -2.04615000 & -2.56410800 \\ \mathrm{~N} & 0.26522400 & -1.13418500 & -1.58989900 \\ \mathrm{H} & 0.52912000 & -2.03848600 & -1.95275200 \\ \mathrm{~N} & -1.51635700 & 0.17080600 & -1.01523900 \\ \mathrm{C} & -2.87943300 & 0.53023200 & -0.96970800 \\ \mathrm{C} & -3.29845100 & 1.72438300 & -1.55499600 \\ \mathrm{C} & -3.80426900 & -0.22343400 & -0.24147500 \\ \mathrm{C} & -4.60687800 & 2.16507900 & -1.39932100 \\ \mathrm{H} & -2.59203500 & 2.29907100 & -2.14208000 \\ \mathrm{C} & -5.10936000 & 0.22319000 & -0.08381400 \\ \mathrm{H} & -3.49266500 & -1.17269000 & 0.17662400 \\ \mathrm{C} & -5.51606300 & 1.42430900 & -0.65430700 \\ \mathrm{H} & -6.53624300 & 1.76945800 & -0.53626800 \\ \mathrm{H} & -0.98853300 & -0.39546800 & 0.79318100 \\ \mathrm{C} & -1.76897600 & -1.31544900 & 2.44602900 \\ \mathrm{C} & -0.73269700 & -0.46741300 & 1.88676600 \\ \mathrm{C} & -0.74969300 & 0.94784800 & 2.45781800 \\ \mathrm{C} & -2.18146600 & 1.47238000 & 2.38610000 \\ \mathrm{C} & -3.14847200 & 0.55310400 & 3.10506600 \\ \mathrm{H} & -0.37830200 & 0.95091200 & 3.48751900 \\ \mathrm{H} & -0.09000200 & 1.57879200 & 1.86204700 \\ \mathrm{H} & -2.26669700 & 2.45827200 & 2.84816900\end{array}$

$\begin{array}{lrrr}\mathrm{H} & -2.48276700 & 1.57113900 & 1.34242900 \\ \mathrm{H} & -3.16385500 & 0.69551200 & 4.18555000 \\ \mathrm{H} & -4.15953400 & 0.61899200 & 2.70843600 \\ \mathrm{O} & -2.82872600 & -0.88961900 & 2.97442900 \\ \mathrm{H} & -1.68863800 & -2.39628000 & 2.43199400 \\ \mathrm{H} & 0.22580300 & -0.98737200 & 1.93838300 \\ \mathrm{C} & -3.24019100 & -3.97598100 & 0.56929300 \\ \mathrm{H} & -4.15114100 & -3.55631800 & 0.12911100 \\ \mathrm{H} & -3.41482200 & -4.15823800 & 1.63220400 \\ \mathrm{H} & -3.02176500 & -4.93274600 & 0.08641000 \\ \mathrm{O} & -2.14644700 & -3.07620200 & 0.45473200 \\ \mathrm{H} & -2.05454800 & -2.85880700 & -0.49495700 \\ \mathrm{H} & -4.91881200 & 3.09075800 & -1.86933900 \\ \mathrm{H} & -5.81873300 & -0.38226500 & 0.47088900 \\ \mathrm{C} & 1.20172800 & -0.39924800 & -0.98528800 \\ \mathrm{C} & 1.75659400 & 0.77505300 & -0.51874400 \\ \mathrm{C} & 2.44970000 & -0.43065000 & -0.40138100 \\ \mathrm{~N} & 1.73723300 & 2.07815000 & -0.28348800 \\ \mathrm{~N} & 3.48383100 & -1.15939700 & -0.02296300 \\ \mathrm{C} & 2.86883800 & 2.64269400 & 0.48608000 \\ \mathrm{C} & 0.87042800 & 3.00172500 & -1.06347200 \\ \mathrm{C} & 4.72650400 & -0.52675200 & 0.46108100 \\ \mathrm{C} & 3.36809300 & -2.63440400 & -0.06387900\end{array}$




$\begin{array}{lrrrrrrr}\mathrm{C} & 3.99285500 & 3.10586700 & -0.43946700 & \mathrm{H} & -1.00508200 & 2.38429300 & -0.22199000 \\ \mathrm{H} & 3.23398600 & 1.81814300 & 1.10356700 & \mathrm{H} & -0.24275800 & 3.73505500 & 0.65912800 \\ \mathrm{C} & 2.41800500 & 3.74146600 & 1.44221600 & \mathrm{H} & -1.02502100 & 4.01688200 & -0.89871000 \\ \mathrm{C} & -0.42878300 & 3.30292700 & -0.32610100 & \mathrm{H} & 1.56818100 & 2.25078100 & -2.98101200 \\ \mathrm{H} & 1.45269800 & 3.92351500 & -1.14006000 & \mathrm{H} & -0.02584700 & 1.62162900 & -2.48541500 \\ \mathrm{C} & 0.62782700 & 2.49514000 & -2.48074700 & \mathrm{H} & 0.13477800 & 3.27916400 & -3.05891400 \\ \mathrm{H} & 4.60076600 & 0.53864100 & 0.26820000 & \mathrm{H} & 5.01838200 & -1.78980700 & 2.21165500 \\ \mathrm{C} & 4.89517600 & -0.73207900 & 1.96387600 & \mathrm{H} & 5.78378200 & -0.20615600 & 2.31908200 \\ \mathrm{C} & 5.93890900 & -0.99068200 & -0.34146000 & \mathrm{H} & 4.02818600 & -0.35013200 & 2.50928600 \\ \mathrm{H} & 4.26488300 & -3.00444300 & 0.43575700 & \mathrm{H} & 5.78939100 & -0.81753400 & -1.40902400 \\ \mathrm{C} & 2.16332900 & -3.11780700 & 0.74179700 & \mathrm{H} & 6.82213900 & -0.43285800 & -0.02399500 \\ \mathrm{C} & 3.37983300 & -3.15431200 & -1.49881400 & \mathrm{H} & 6.15050300 & -2.05171800 & -0.18739900 \\ \mathrm{H} & 4.29666100 & 2.30966300 & -1.12432000 & \mathrm{H} & 2.17755600 & -4.20710700 & 0.81188100 \\ \mathrm{H} & 3.68495000 & 3.96508600 & -1.04039700 & \mathrm{H} & 2.19802000 & -2.71238700 & 1.75669800 \\ \mathrm{H} & 4.86468900 & 3.40830200 & 0.14529100 & \mathrm{H} & 1.21300800 & -2.83514400 & 0.28009300 \\ \mathrm{H} & 2.03443900 & 4.61603500 & 0.91242900 & \mathrm{H} & 2.54204200 & -2.75925200 & -2.07936800 \\ \mathrm{H} & 1.64534000 & 3.38106800 & 2.12464900 & \mathrm{H} & 4.30061400 & -2.86984500 & -2.01038300 \\ \mathrm{H} & 3.27175800 & 4.06880400 & 2.03898000 & \mathrm{H} & 3.30339300 & -4.24333100 & -1.50780200\end{array}$

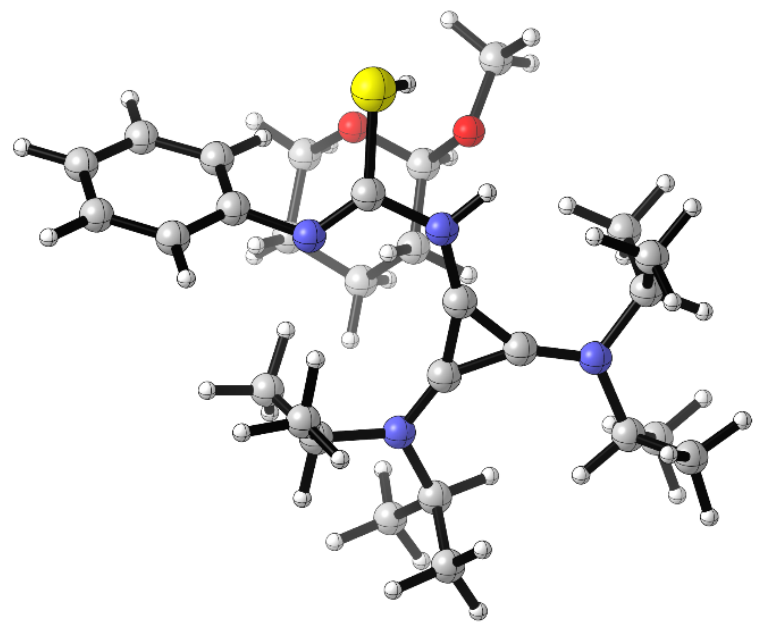

Figure S44. Optimized Structure of $Z, E-\mathbf{1}-A n-I_{N} \mathbf{T}_{4}$

- Thermochemistry -

(0 imaginary frequencies)

Zero-point correction $=$

Thermal correction to Energy=

0.726739 (Hartree/Particle)

Thermal correction to Enthalpy=

0.766823

Thermal correction to Gibbs Free Energy $=0.654513$

Sum of electronic and zero-point Energies $=\quad-1861.893184$

Sum of electronic and thermal Energies $=\quad-1861.853101$

Sum of electronic and thermal Enthalpies $=\quad-1861.852156$

Sum of electronic and thermal Free Energies $=\quad-1861.965410$ 


$\begin{array}{lrrr}\mathrm{N} & 0.10222900 & -0.78028900 & -1.68569500 \\ \mathrm{H} & 0.41578500 & -1.67284700 & -2.03375200 \\ \mathrm{~N} & -1.74267800 & 0.54036800 & -1.35625800 \\ \mathrm{C} & -3.10932900 & 0.84194900 & -1.19497100 \\ \mathrm{C} & -3.61206800 & 1.98086000 & -1.82055900 \\ \mathrm{C} & -3.92432300 & 0.09450100 & -0.34543600 \\ \mathrm{C} & -4.93611000 & 2.34863600 & -1.62522600 \\ \mathrm{H} & -2.96390200 & 2.56349100 & -2.46474700 \\ \mathrm{C} & -5.24242300 & 0.48075600 & -0.14468800 \\ \mathrm{H} & -3.51955200 & -0.77543400 & 0.15823200 \\ \mathrm{C} & -5.75458900 & 1.60222000 & -0.78539800 \\ \mathrm{H} & -6.78590300 & 1.89483700 & -0.62927500 \\ \mathrm{H} & -0.60601400 & -0.45848800 & 0.93110300 \\ \mathrm{C} & -1.04561800 & -2.34308900 & 1.79752600 \\ \mathrm{C} & -0.35758200 & -0.99239400 & 1.85173800 \\ \mathrm{C} & -0.87220200 & -0.19995200 & 3.05477800 \\ \mathrm{C} & -2.39931500 & -0.12779700 & 3.01354100 \\ \mathrm{C} & -2.98903000 & -1.52398900 & 2.85117000 \\ \mathrm{H} & -0.54966300 & -0.68852400 & 3.98193800 \\ \mathrm{H} & -0.43851600 & 0.80274200 & 3.05742900 \\ \mathrm{H} & -2.79349800 & 0.32667800 & 3.92617300 \\ \mathrm{H} & -2.71997500 & 0.49474700 & 2.17183200 \\ \mathrm{H} & -2.80170200 & -2.12650000 & 3.75227100 \\ \mathrm{H} & -4.06579200 & -1.48871500 & 2.68589800 \\ \mathrm{O} & -2.44338200 & -2.19061600 & 1.71667200 \\ \mathrm{H} & -0.80551600 & -2.92648200 & 2.70637400 \\ \mathrm{H} & 0.72620600 & -1.13664000 & 1.89316000 \\ \mathrm{C} & -0.96314100 & -4.42888400 & 0.70992100 \\ \mathrm{H} & -2.04409000 & -4.56869600 & 0.78866800 \\ \mathrm{H} & -0.47013800 & -4.91841900 & 1.55685900 \\ \mathrm{H} & -0.59966300 & -4.86972100 & -0.21798500 \\ \mathrm{O} & -0.63019100 & -3.04559200 & 0.66955200 \\ \mathrm{H} & -1.86232700 & -2.70618700 & -1.12308800 \\ \mathrm{H} & -5.32846200 & 3.22557900 & -2.12664500 \\ \mathrm{H} & -5.87507400 & -0.10471000 & 0.51270700 \\ \mathrm{C} & 1.02442400 & -0.02647700 & -1.05451500 \\ \mathrm{C} & 1.48382000 & 1.06677500 & -0.34891900 \\ \mathrm{C} & 2.33109700 & -0.01048300 & -0.63726700 \\ \mathrm{~N} & 1.35884500 & 2.22922400 & 0.26116400 \\ \mathrm{~N} & 3.49457000 & -0.62608800 & -0.56604500\end{array}$

$\begin{array}{lrrr}\mathrm{C} & 2.47685600 & 2.68110000 & 1.12173100 \\ \mathrm{C} & 0.23748000 & 3.16680500 & -0.00368100 \\ \mathrm{C} & 4.70042000 & 0.08143700 & -0.08730500 \\ \mathrm{C} & 3.58403500 & -2.04970900 & -0.96153100 \\ \mathrm{C} & 3.35914600 & 3.68945400 & 0.39099600 \\ \mathrm{H} & 3.05992600 & 1.78281400 & 1.33254400 \\ \mathrm{C} & 1.97846400 & 3.19924400 & 2.46738200 \\ \mathrm{C} & -0.99845500 & 2.80105600 & 0.81348400 \\ \mathrm{H} & 0.60730100 & 4.13011500 & 0.35326900 \\ \mathrm{C} & -0.01629200 & 3.31583300 & -1.49892400 \\ \mathrm{H} & 4.40658800 & 1.12755100 & 0.00098100 \\ \mathrm{C} & 5.12523500 & -0.42931500 & 1.28581100 \\ \mathrm{C} & 5.82563800 & 0.02147000 & -1.11720200 \\ \mathrm{H} & 4.60877100 & -2.34258400 & -0.72904800 \\ \mathrm{C} & 2.65519700 & -2.91380300 & -0.11390600 \\ \mathrm{C} & 3.38382000 & -2.22426100 & -2.46429300 \\ \mathrm{H} & 3.72328100 & 3.28228100 & -0.55608500 \\ \mathrm{H} & 2.81385500 & 4.61137300 & 0.17379400 \\ \mathrm{H} & 4.22180000 & 3.95362100 & 1.00692400 \\ \mathrm{H} & 1.40235700 & 4.12180500 & 2.36884900 \\ \mathrm{H} & 1.36051300 & 2.45277400 & 2.97017500 \\ \mathrm{H} & 2.83704900 & 3.41807500 & 3.10547000 \\ \mathrm{H} & -1.29945800 & 1.77360000 & 0.61793800 \\ \mathrm{H} & -0.80967500 & 2.92083300 & 1.88190800 \\ \mathrm{H} & -1.83482800 & 3.44733600 & 0.53911300 \\ \mathrm{H} & 0.90122300 & 3.60508500 & -2.01748800 \\ \mathrm{H} & -0.40750400 & 2.39377600 & -1.92862500 \\ \mathrm{H} & -0.75971700 & 4.09910300 & -1.66065700 \\ \mathrm{H} & 5.42107400 & -1.48115000 & 1.24512100 \\ \mathrm{H} & 5.98281500 & 0.13982800 & 1.65040700 \\ \mathrm{H} & 4.31299700 & -0.33159400 & 2.01076200 \\ \mathrm{H} & 5.48903400 & 0.39649300 & -2.08586400 \\ \mathrm{H} & 6.65658800 & 0.64463100 & -0.78067800 \\ \mathrm{H} & 6.21306400 & -0.99159600 & -1.24982800 \\ \mathrm{H} & 2.80336800 & -3.96773700 & -0.35913400 \\ \mathrm{H} & 2.87234400 & -2.77987200 & 0.94849300 \\ \mathrm{H} & 1.59716700 & -2.68308700 & -0.26361700 \\ \mathrm{H} & 2.39101500 & -1.89945200 & -2.78838600 \\ \mathrm{H} & 4.11812400 & -1.64398300 & -3.02563400 \\ \mathrm{H} & 3.49475900 & -3.27522100 & -2.73806900\end{array}$

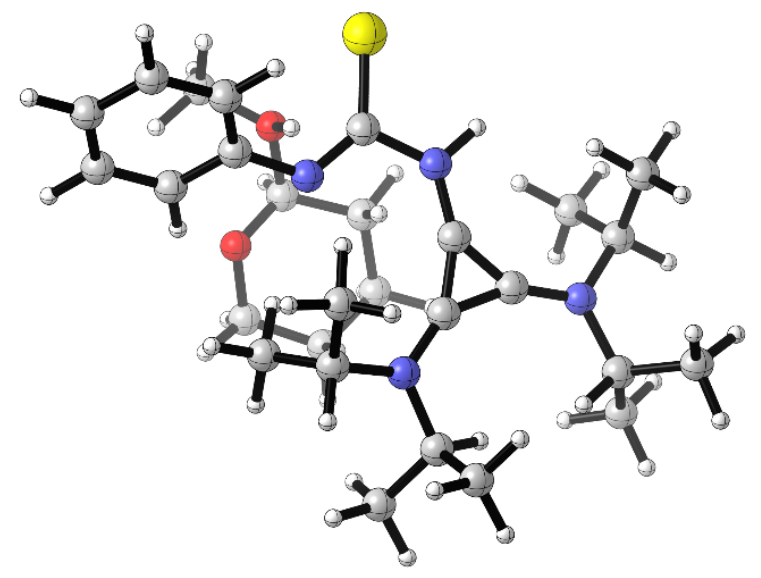

Figure S45. Optimized Structure of $Z, E-1-A n-T_{3}-\mathbf{P A}$. 
- Thermochemistry -

$(1$ imaginary frequency $=-363.40 \mathrm{~Hz})$

Zero-point correction=

Thermal correction to Energy=

0.729072 (Hartree/Particle)

Thermal correction to Enthalpy=

0.768091

Thermal correction to Gibbs Free Energy $=\quad 0.658830$

Sum of electronic and zero-point Energies=

$-1861.845824$

Sum of electronic and thermal Energies $=$

$-1861.806806$

Sum of electronic and thermal Enthalpies=

$-1861.805862$

Sum of electronic and thermal Free Energies=

$-1861.916067$

$\begin{array}{lrrr}\mathrm{C} & -1.33445100 & -1.20724200 & -1.32616800 \\ \mathrm{~S} & -2.13874200 & -2.63496800 & -1.80507400 \\ \mathrm{~N} & 0.08456300 & -1.32572900 & -1.33168400 \\ \mathrm{H} & 0.40569100 & -2.23978300 & -1.61672000 \\ \mathrm{~N} & -1.77518000 & -0.08595600 & -0.83853900 \\ \mathrm{C} & -3.09778800 & 0.35239300 & -1.05375600 \\ \mathrm{C} & -3.73033500 & 0.24379500 & -2.29774500 \\ \mathrm{C} & -3.76287100 & 1.02510600 & -0.02435100 \\ \mathrm{C} & -4.99659700 & 0.77413400 & -2.48984300 \\ \mathrm{H} & -3.22680800 & -0.26131000 & -3.11205900 \\ \mathrm{C} & -5.03602200 & 1.54688200 & -0.22141500 \\ \mathrm{H} & -3.27209000 & 1.13224100 & 0.93401700 \\ \mathrm{C} & -5.66179900 & 1.42230700 & -1.45409500 \\ \mathrm{H} & -6.65093900 & 1.83519400 & -1.61189400 \\ \mathrm{H} & -0.26379900 & -1.33515200 & 1.10655500 \\ \mathrm{C} & -1.90874100 & -1.45982900 & 2.42991200 \\ \mathrm{C} & -0.46303800 & -1.75636000 & 2.09352800 \\ \mathrm{C} & 0.45099600 & -1.08323000 & 3.11991700 \\ \mathrm{C} & 0.09949100 & 0.40054400 & 3.21993000 \\ \mathrm{C} & -1.37881800 & 0.55809200 & 3.53611200 \\ \mathrm{H} & 0.33557300 & -1.56161100 & 4.09916100 \\ \mathrm{H} & 1.49226700 & -1.22218700 & 2.82232000 \\ \mathrm{H} & 0.68424900 & 0.88983900 & 4.00315700 \\ \mathrm{H} & 0.31836900 & 0.90250100 & 2.27142000 \\ \mathrm{H} & -1.60574200 & 0.16430900 & 4.53600000 \\ \mathrm{H} & -1.70287300 & 1.59639400 & 3.49167900 \\ \mathrm{O} & -2.19273100 & -0.12035600 & 2.57091200 \\ \mathrm{H} & -2.27049700 & -2.04534000 & 3.28569000 \\ \mathrm{H} & -0.31221100 & -2.83640300 & 2.04782200 \\ \mathrm{C} & -4.18814200 & -1.92295800 & 1.41910100 \\ \mathrm{H} & -4.51432100 & -0.92337000 & 1.69153500 \\ \mathrm{H} & -4.43913900 & -2.67545500 & 2.16224100 \\ \mathrm{H} & -4.53705900 & -2.20324700 & 0.42811800 \\ \mathrm{O} & -2.72244400 & -1.93134700 & 1.31260900 \\ \mathrm{H} & -2.41178700 & -1.56860100 & 0.43737900 \\ \mathrm{H} & -5.46902000 & 0.67879900 & -3.46082200 \\ \mathrm{H} & -5.53431200 & 2.06269900 & 0.59236300 \\ \mathrm{C} & 1.01325700 & -0.44053500 & -0.94641500 \\ \mathrm{C} & 1.50909900 & 0.79144000 & -0.56061400 \\ \mathrm{C} & 2.35206500 & -0.31646500 & -0.65314300 \\ \mathrm{~N} & 1.40297100 & 2.07292800 & -0.25686800\end{array}$

$\begin{array}{lrrr}\mathrm{N} & 3.53421900 & -0.89812100 & -0.54348400 \\ \mathrm{C} & 2.59009400 & 2.74764700 & 0.31267100 \\ \mathrm{C} & 0.22853400 & 2.89386100 & -0.64891700 \\ \mathrm{C} & 4.75711300 & -0.09652300 & -0.33896500 \\ \mathrm{C} & 3.62138100 & -2.37249000 & -0.61545900 \\ \mathrm{C} & 3.36208400 & 3.50202900 & -0.76740400 \\ \mathrm{H} & 3.21901300 & 1.94557500 & 0.70362000 \\ \mathrm{C} & 2.22756000 & 3.63249100 & 1.50145400 \\ \mathrm{C} & -0.87215800 & 2.79381400 & 0.40085000 \\ \mathrm{H} & 0.60811100 & 3.91809600 & -0.66601600 \\ \mathrm{C} & -0.24248200 & 2.56347300 & -2.06009300 \\ \mathrm{H} & 4.45297800 & 0.93881900 & -0.49323400 \\ \mathrm{C} & 5.27754700 & -0.25039300 & 1.08705900 \\ \mathrm{C} & 5.81602900 & -0.40582600 & -1.39410000 \\ \mathrm{H} & 4.65421300 & -2.60884400 & -0.35450500 \\ \mathrm{C} & 2.72655800 & -3.03177500 & 0.43171300 \\ \mathrm{C} & 3.36681300 & -2.88072900 & -2.03199000 \\ \mathrm{H} & 3.62936700 & 2.84006400 & -1.59536400 \\ \mathrm{H} & 2.77158300 & 4.32707200 & -1.17331500 \\ \mathrm{H} & 4.27998900 & 3.92477800 & -0.35249800 \\ \mathrm{H} & 1.60836100 & 4.48343400 & 1.21009900 \\ \mathrm{H} & 1.69819200 & 3.06250100 & 2.26771200 \\ \mathrm{H} & 3.14332100 & 4.03093600 & 1.94292000 \\ \mathrm{H} & -1.15792900 & 1.74973100 & 0.52704300 \\ \mathrm{H} & -0.54116100 & 3.20529400 & 1.35733300 \\ \mathrm{H} & -1.75539800 & 3.34685300 & 0.07505600 \\ \mathrm{H} & 0.58210500 & 2.64092700 & -2.77340600 \\ \mathrm{H} & -0.68021600 & 1.56621800 & -2.10832200 \\ \mathrm{H} & -1.01634600 & 3.27441900 & -2.35530800 \\ \mathrm{H} & 5.57358800 & -1.28279900 & 1.29237500 \\ \mathrm{H} & 6.15490200 & 0.38119200 & 1.24119400 \\ \mathrm{H} & 4.51422700 & 0.03729500 & 1.81503400 \\ \mathrm{H} & 5.40836500 & -0.28983200 & -2.40020500 \\ \mathrm{H} & 6.65036000 & 0.28946800 & -1.28222500 \\ \mathrm{H} & 6.21918100 & -1.41649200 & -1.29380800 \\ \mathrm{H} & 2.88544600 & -4.11177900 & 0.42934300 \\ \mathrm{H} & 2.96033700 & -2.65333300 & 1.42976200 \\ \mathrm{H} & 1.66554100 & -2.85603300 & 0.23514500 \\ \mathrm{H} & 2.36382800 & -2.62438500 & -2.38421200 \\ \mathrm{H} & 4.08290900 & -2.45162200 & -2.73466300 \\ \mathrm{H} & 3.46254000 & -3.96773500 & -2.06712000\end{array}$




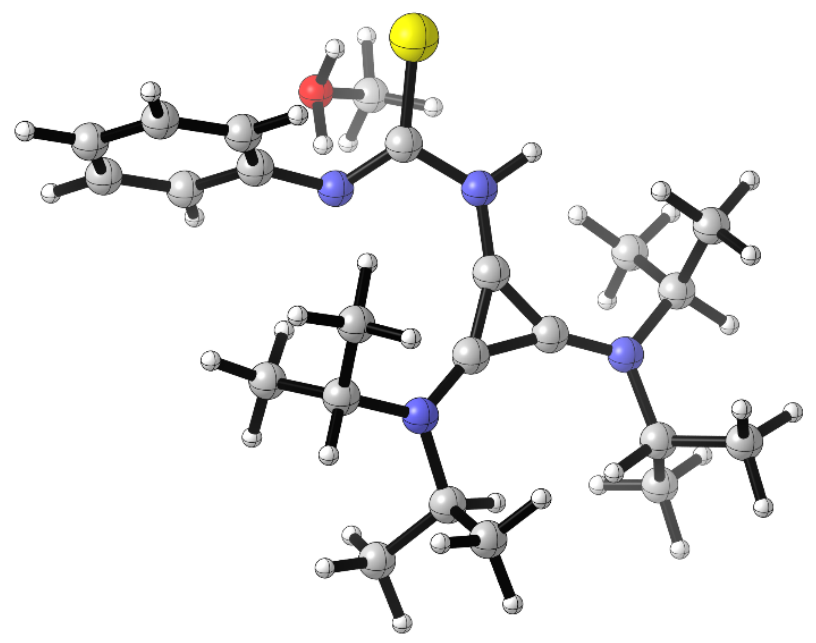

Figure S46. Optimized Structure of $Z, E-1-A n-S_{3}-M A$.

- Thermochemistry -

$(1$ imaginary frequency $=-453.03 \mathrm{~Hz})$

Zero-point correction $=$

Thermal correction to Energy=

Thermal correction to Enthalpy=

Thermal correction to Gibbs Free Energy=

Sum of electronic and zero-point Energies=

Sum of electronic and thermal Energies=

Sum of electronic and thermal Enthalpies=

Sum of electronic and thermal Free Energies=
0.599373 (Hartree/Particle)

0.632729

0.633673

0.534554

$-1591.411199$

$-1591.377843$

$-1591.376899$

$-1591.476018$

$\begin{array}{lrrr}\mathrm{C} & -1.55083000 & -1.41943300 & -0.58610800 \\ \mathrm{~S} & -2.38483800 & -2.68327400 & -1.35892200 \\ \mathrm{~N} & -0.15787700 & -1.48825700 & -0.66253900 \\ \mathrm{H} & 0.19748700 & -2.33096600 & -1.09205600 \\ \mathrm{~N} & -2.01715400 & -0.48624400 & 0.23549800 \\ \mathrm{C} & -3.28692400 & 0.11618100 & 0.02210700 \\ \mathrm{C} & -3.82224700 & 0.35419300 & -1.24576300 \\ \mathrm{C} & -3.96844700 & 0.59758400 & 1.14229200 \\ \mathrm{C} & -5.01746100 & 1.04583500 & -1.37856400 \\ \mathrm{H} & -3.31121000 & -0.00681500 & -2.12943700 \\ \mathrm{C} & -5.16692000 & 1.28215000 & 1.00318200 \\ \mathrm{H} & -3.54043100 & 0.44860500 & 2.12851800 \\ \mathrm{C} & -5.69866000 & 1.51027400 & -0.25938600 \\ \mathrm{H} & -6.63400900 & 2.04488200 & -0.37162000 \\ \mathrm{O} & -3.02755400 & -2.37954300 & 1.58810900 \\ \mathrm{H} & -3.20939500 & -2.75752700 & 0.67649900 \\ \mathrm{H} & -5.42226000 & 1.21887500 & -2.36905700 \\ \mathrm{H} & -5.68175700 & 1.64408600 & 1.88552500 \\ \mathrm{C} & 0.75142400 & -0.54310900 & -0.32622400 \\ \mathrm{C} & 1.19596700 & 0.74159100 & -0.08952800\end{array}$

$\begin{array}{lrrc}\mathrm{C} & 2.09193900 & -0.33937500 & -0.12073400 \\ \mathrm{~N} & 1.03188600 & 2.03652100 & 0.05892900 \\ \mathrm{~N} & 3.30874500 & -0.84048600 & -0.04900100 \\ \mathrm{C} & 2.19688300 & 2.85104900 & 0.47832900 \\ \mathrm{C} & -0.22606900 & 2.72064500 & -0.34192800 \\ \mathrm{C} & 4.49044700 & 0.04404800 & 0.04268900 \\ \mathrm{C} & 3.48474400 & -2.30987800 & -0.03604700 \\ \mathrm{C} & 2.86798100 & 3.49672300 & -0.73132400 \\ \mathrm{H} & 2.89059800 & 2.14499500 & 0.93888100 \\ \mathrm{C} & 1.82917300 & 3.86569300 & 1.55566800 \\ \mathrm{C} & -1.24230000 & 2.70122200 & 0.79377700 \\ \mathrm{H} & 0.07473100 & 3.75349400 & -0.53133300 \\ \mathrm{C} & -0.76303000 & 2.16123700 & -1.65384100 \\ \mathrm{H} & 4.11303900 & 1.04759900 & -0.15258400 \\ \mathrm{C} & 5.08922400 & 0.00775100 & 1.44528800 \\ \mathrm{C} & 5.51098000 & -0.26718500 & -1.04830300 \\ \mathrm{H} & 4.53547400 & -2.46775600 & 0.21163400 \\ \mathrm{C} & 2.65410900 & -2.95929600 & 1.06750500 \\ \mathrm{C} & 3.22919300 & -2.91340800 & -1.41452700 \\ \mathrm{H} & 3.14788000 & 2.74677000 & -1.47605100\end{array}$




$\begin{array}{rrrrrrrr}\mathrm{H} & 2.20422200 & 4.21905200 & -1.21294000 & \mathrm{H} & 5.05324200 & -0.21940600 & -2.03835400 \\ \mathrm{H} & 3.77004700 & 4.02983200 & -0.42316500 & \mathrm{H} & 6.31453000 & 0.47068600 & -1.00907900 \\ \mathrm{H} & 1.14628600 & 4.63350900 & 1.18560600 & \mathrm{H} & 5.96726900 & -1.25173600 & -0.92002100 \\ \mathrm{H} & 1.37196900 & 3.37762500 & 2.41798700 & \mathrm{H} & 2.89588700 & -4.02160700 & 1.13523100 \\ \mathrm{H} & 2.73766800 & 4.37006800 & 1.89076000 & \mathrm{H} & 2.86390500 & -2.49620900 & 2.03395000 \\ \mathrm{H} & -1.42046600 & 1.67435700 & 1.11035000 & \mathrm{H} & 1.58135800 & -2.87680200 & 0.87164200 \\ \mathrm{H} & -0.89301900 & 3.28391200 & 1.64733500 & \mathrm{H} & 2.20498000 & -2.73107700 & -1.75300200 \\ \mathrm{H} & -2.19246000 & 3.12010300 & 0.45590900 & \mathrm{H} & 3.90356500 & -2.49051300 & -2.16052600 \\ \mathrm{H} & -0.00240900 & 2.20175500 & -2.43741000 & \mathrm{H} & 3.38205000 & -3.99405600 & -1.38776700 \\ \mathrm{H} & -1.10978800 & 1.13452000 & -1.54114000 & \mathrm{H} & -2.49061500 & -1.50251300 & 1.25980300 \\ \mathrm{H} & -1.62093200 & 2.75431800 & -1.97429900 & \mathrm{C} & -2.19946000 & -3.28814200 & 2.35647500 \\ \mathrm{H} & 5.48166800 & -0.98374400 & 1.68679600 & \mathrm{H} & -2.76641700 & -4.20475000 & 2.49863300 \\ \mathrm{H} & 5.91739500 & 0.71575700 & 1.51800200 & \mathrm{H} & -1.26847000 & -3.48607000 & 1.82433000 \\ \mathrm{H} & 4.34215200 & 0.27367200 & 2.19717100 & \mathrm{H} & -2.01342500 & -2.80703300 & 3.31272700\end{array}$

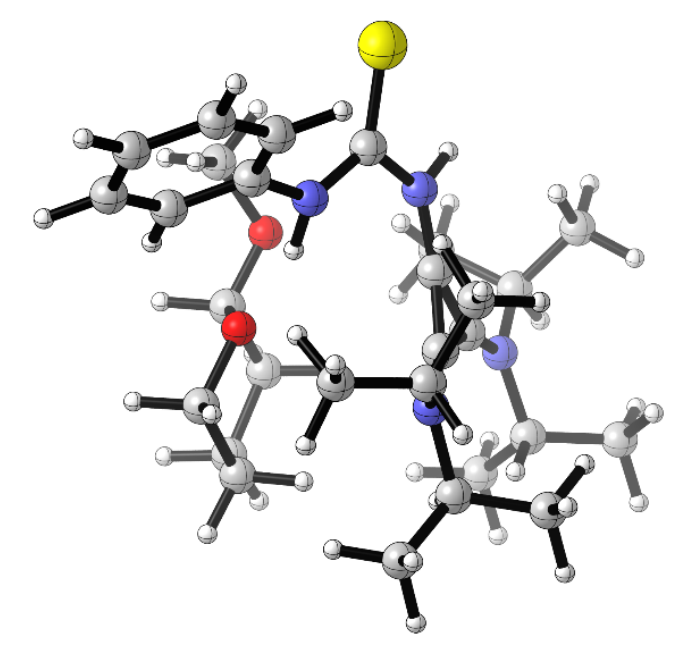

Figure S47. Optimized Structure of Z,E-1-An-INT 5 .

- Thermochemistry -

(0 imaginary frequencies)

Zero-point correction $=$

Thermal correction to Energy=

0.731368 (Hartree/Particle)

Thermal correction to Enthalpy=

0.770700

Thermal correction to Gibbs Free Energy $=\quad 0.660142$

Sum of electronic and zero-point Energies=

$-1861.915232$

Sum of electronic and thermal Energies=

$-1861.875899$

Sum of electronic and thermal Enthalpies=

$-1861.874955$

Sum of electronic and thermal Free Energies=

$-1861.986458$

$\begin{array}{lrrr}\mathrm{C} & -1.70186600 & -1.56610100 & -1.24336900 \\ \mathrm{~S} & -2.56040900 & -2.67232000 & -2.11256100 \\ \mathrm{~N} & -0.29918900 & -1.60871200 & -1.29742400 \\ \mathrm{H} & 0.07300100 & -2.48747100 & -1.62488400 \\ \mathrm{~N} & -2.15248900 & -0.59793900 & -0.42181500 \\ \mathrm{C} & -3.50088100 & -0.19631100 & -0.23509100\end{array}$

$\begin{array}{lrrr}\mathrm{C} & -4.36384000 & 0.00599000 & -1.30743700 \\ \mathrm{C} & -3.91667200 & 0.09090600 & 1.06299500 \\ \mathrm{C} & -5.64762200 & 0.47483900 & -1.06931200 \\ \mathrm{H} & -4.04036500 & -0.20916800 & -2.31672400 \\ \mathrm{C} & -5.20045600 & 0.56850300 & 1.28853500 \\ \mathrm{H} & -3.22808600 & -0.05351600 & 1.88786200\end{array}$




\begin{tabular}{|c|c|c|c|c|c|c|c|}
\hline $\mathrm{C}$ & -6.07174400 & 0.75722300 & 0.22423900 & $\mathrm{H}$ & 2.39931500 & 2.34804700 & 0.21848700 \\
\hline $\mathrm{H}$ & -7.07590500 & 1.12347700 & 0.39975400 & $\mathrm{C}$ & 1.30258900 & 4.15418900 & 0.24661400 \\
\hline $\mathrm{H}$ & 1.88057500 & -0.71860800 & 1.94301700 & $\mathrm{C}$ & -1.68477200 & 2.83644900 & -0.56283700 \\
\hline $\mathrm{C}$ & -0.08885300 & -1.09865700 & 2.62824000 & $\mathrm{H}$ & -0.17415800 & 3.49926900 & -1.91482600 \\
\hline $\mathrm{C}$ & 1.37446100 & -0.81512700 & 2.90839200 & $\mathrm{C}$ & -0.94861800 & 1.70246800 & -2.71450000 \\
\hline $\mathrm{C}$ & 1.52960000 & 0.47064800 & 3.72134200 & $\mathrm{H}$ & 3.79392700 & 1.17245100 & -0.39678300 \\
\hline $\mathrm{C}$ & 0.79365900 & 1.61801700 & 3.03101100 & $\mathrm{C}$ & 4.88536800 & -0.03494100 & 0.98030200 \\
\hline $\mathrm{C}$ & -0.64633700 & 1.22075800 & 2.74643300 & $\mathrm{C}$ & 5.24512600 & 0.12073700 & -1.53638800 \\
\hline $\mathrm{H}$ & 1.11458800 & 0.32298500 & 4.72498800 & $\mathrm{H}$ & 4.47387500 & -2.30919400 & -0.55812100 \\
\hline $\mathrm{H}$ & 2.58712100 & 0.71023800 & 3.85502200 & $\mathrm{C}$ & 2.67920200 & -3.03562100 & 0.32093500 \\
\hline $\mathrm{H}$ & 0.81267800 & 2.52485400 & 3.64144200 & $\mathrm{C}$ & 3.13039600 & -2.67147000 & -2.16680800 \\
\hline $\mathrm{H}$ & 1.28318800 & 1.84824200 & 2.07909600 & $\mathrm{H}$ & 2.93336700 & 2.32486900 & -2.23576600 \\
\hline $\mathrm{H}$ & -1.19530500 & 1.07045300 & 3.68688500 & $\mathrm{H}$ & 2.00258800 & 3.81438000 & -2.44679700 \\
\hline $\mathrm{H}$ & -1.17486400 & 1.97440400 & 2.16244600 & $\mathrm{H}$ & 3.46714100 & 3.82671700 & -1.46427300 \\
\hline $\mathrm{O}$ & -0.69353600 & 0.02309400 & 1.97491200 & $\mathrm{H}$ & 0.68649500 & 4.79763000 & -0.38500400 \\
\hline $\mathrm{H}$ & -0.63666000 & -1.27191300 & 3.57047300 & $\mathrm{H}$ & 0.73820600 & 3.90172100 & 1.14562300 \\
\hline $\mathrm{H}$ & 1.80701500 & -1.67389600 & 3.42647600 & $\mathrm{H}$ & 2.17686300 & 4.73469700 & 0.54763900 \\
\hline $\mathrm{C}$ & -1.43712900 & -2.89058100 & 1.87438800 & $\mathrm{H}$ & -2.04157000 & 1.92614500 & -0.08426000 \\
\hline $\mathrm{H}$ & -2.29589200 & -2.23544700 & 1.70509000 & $\mathrm{H}$ & -1.39343300 & 3.54809200 & 0.21066500 \\
\hline $\mathrm{H}$ & -1.52954400 & -3.37515700 & 2.85157500 & $\mathrm{H}$ & -2.52354400 & 3.26480600 & -1.11582100 \\
\hline $\mathrm{H}$ & -1.41872800 & -3.65076000 & 1.09533500 & $\mathrm{H}$ & -0.09673900 & 1.48070900 & -3.36199700 \\
\hline $\mathrm{O}$ & -0.20683600 & -2.18007900 & 1.78288000 & $\mathrm{H}$ & -1.42308400 & 0.76860900 & -2.41498700 \\
\hline $\mathrm{H}$ & -1.52095200 & -0.30683400 & 0.32733200 & $\mathrm{H}$ & -1.67953500 & 2.26124800 & -3.30133200 \\
\hline $\mathrm{H}$ & -6.32192000 & 0.62213900 & -1.90455500 & $\mathrm{H}$ & 5.34940200 & -1.02145600 & 1.05849800 \\
\hline $\mathrm{H}$ & -5.52017500 & 0.78556800 & 2.30086300 & $\mathrm{H}$ & 5.66643400 & 0.70974900 & 1.14660700 \\
\hline $\mathrm{C}$ & 0.57182000 & -0.63070200 & -0.99393400 & $\mathrm{H}$ & 4.14269100 & 0.05910200 & 1.77615600 \\
\hline $\mathrm{C}$ & 0.92792600 & 0.68931700 & -0.83526000 & $\mathrm{H}$ & 4.75192200 & 0.29407200 & -2.49554800 \\
\hline $\mathrm{C}$ & 1.88820500 & -0.32678400 & -0.74402300 & $\mathrm{H}$ & 6.00597100 & 0.89191200 & -1.39970000 \\
\hline $\mathrm{N}$ & 0.65363700 & 1.98394500 & -0.80758100 & $\mathrm{H}$ & 5.76012700 & -0.84193200 & -1.58210200 \\
\hline $\mathrm{N}$ & 3.12276600 & -0.75992700 & -0.58990600 & $\mathrm{H}$ & 2.88535800 & -4.09594100 & 0.16161600 \\
\hline $\mathrm{C}$ & 1.77277600 & 2.90198900 & -0.48463100 & $\mathrm{H}$ & 3.02606600 & -2.76911500 & 1.32096700 \\
\hline $\mathrm{C}$ & -0.53123400 & 2.54707300 & -1.51562400 & $\mathrm{H}$ & 1.59676200 & -2.89016300 & 0.30620900 \\
\hline $\mathrm{C}$ & 4.24346300 & 0.18108700 & -0.38685900 & $\mathrm{H}$ & 2.07679800 & -2.56602300 & -2.44016800 \\
\hline $\mathrm{C}$ & 3.40231800 & -2.21028300 & -0.73678000 & $\mathrm{H}$ & 3.71775100 & -2.09259400 & -2.88198200 \\
\hline $\mathrm{C}$ & 2.59033300 & 3.23281400 & -1.73223600 & $\mathrm{H}$ & 3.39437800 & -3.72453400 & -2.27879400 \\
\hline
\end{tabular}




\section{b. Cyclopropenium N(3) Protonation}

Scheme S3. Pathway 2: Brønsted Acid Catalysis Featuring Conformer E,Z-1.

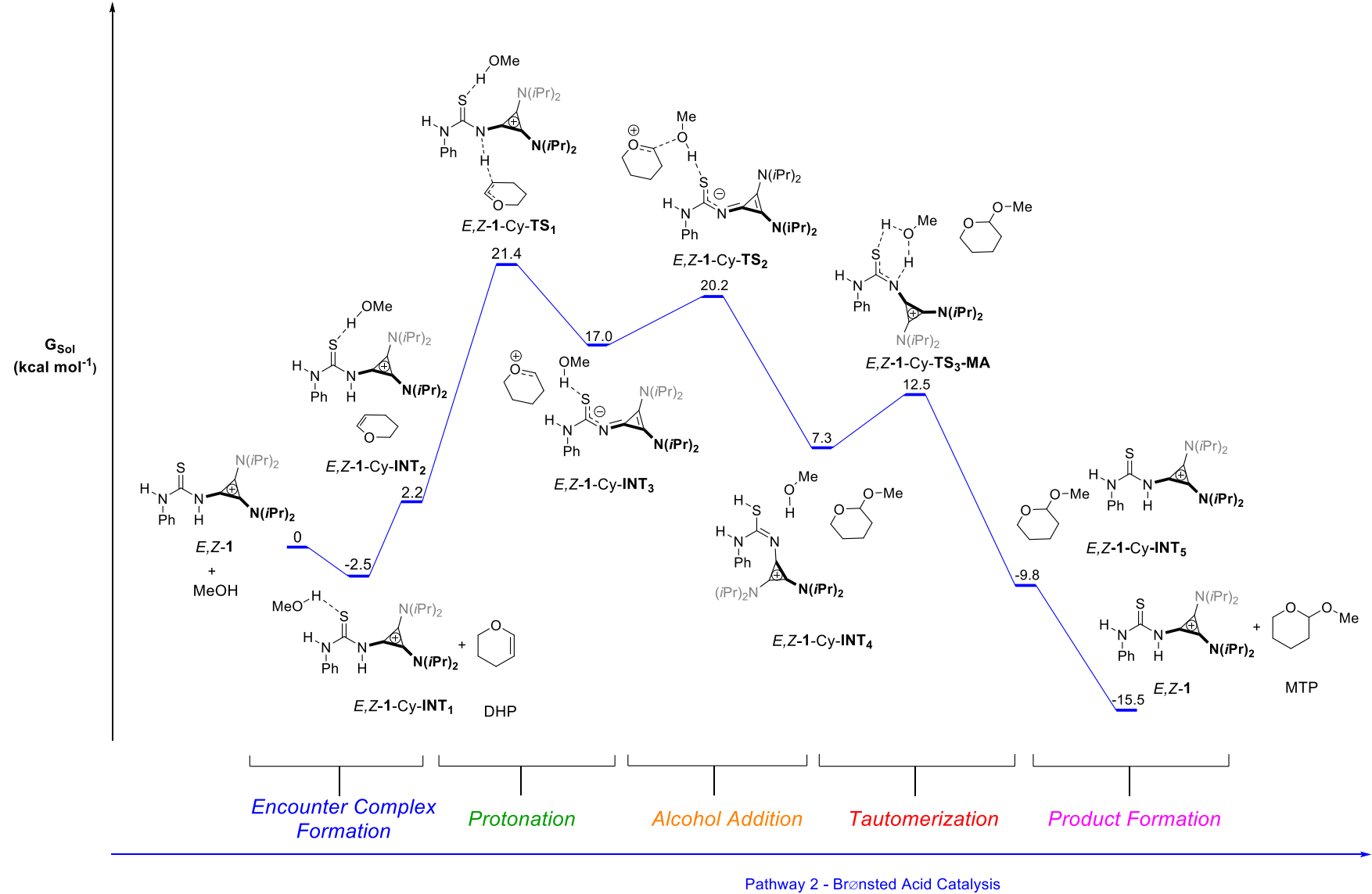

Table S2. Relative Energies for Pathway 2: Brønsted Acid Catalysis Featuring Conformer E,Z-1.

\begin{tabular}{|c|c|c|c|c|c|c|c|c|c|c|c|}
\hline & \multicolumn{10}{|c|}{$\Delta \mathrm{G}_{\text {sol }}(\mathrm{kcal} / \mathrm{mol})$} \\
& eq. & & & & & & & $\mathrm{INT}$ & \\
& & & $\mathrm{INT}_{2}$ & $\mathrm{TS}_{1}$ & $\mathrm{INT}_{3}$ & $\mathrm{TS}_{2}$ & $\mathrm{INT}_{4}$ & $\mathrm{TS}_{3}$ & $\mathrm{TS}_{3}$ & $\mathrm{INT}_{5}$ & Prod. \\
\hline E, Z & 0.0 & -2.5 & 2.2 & 21.4 & 17.0 & 20.2 & 7.3 & 19.2 & 12.5 & -9.8 & -15.5 \\
\hline
\end{tabular}

* "Cy" added to names in SI to differentiate modes of catalysis. 


\section{i. E,Z Conformation}

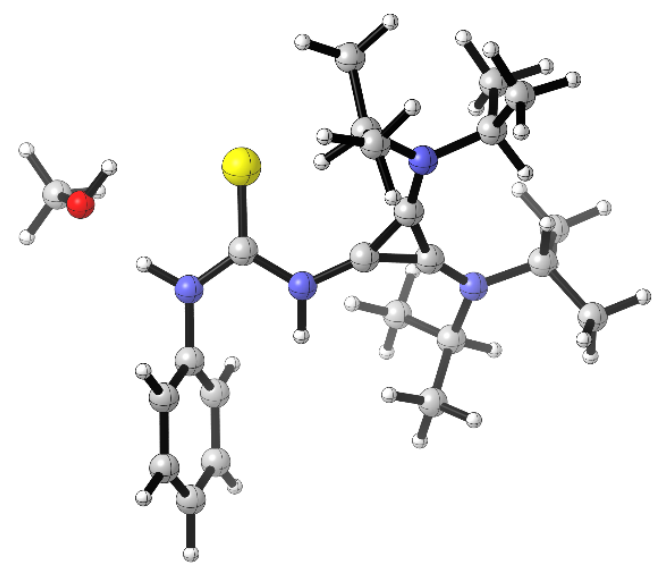

Figure S48. Optimized Structure of E,Z-1-Cy-INT . $_{\text {. }}$

- Thermochemistry -

(0 imaginary frequencies)

Zero-point correction=

Thermal correction to Energy=

Thermal correction to Enthalpy=

Thermal correction to Gibbs Free Energy=

Sum of electronic and zero-point Energies=

Sum of electronic and thermal Energies=

Sum of electronic and thermal Enthalpies=

Sum of electronic and thermal Free Energies=
0.602056 (Hartree/Particle)

0.636847

0.637792

0.533221

$-1591.463325$

$-1591.428534$

$-1591.427590$

$-1591.532161$

$\begin{array}{lrrr}\mathrm{C} & -1.69895000 & -0.94482800 & 0.27324700 \\ \mathrm{~S} & -0.99553400 & -2.23085900 & 1.08507800 \\ \mathrm{~N} & -3.01681100 & -0.77676700 & 0.14122900 \\ \mathrm{H} & -3.60683100 & -1.53307000 & 0.52637900 \\ \mathrm{~N} & -0.93408200 & 0.06200400 & -0.29718200 \\ \mathrm{C} & -3.63938800 & 0.33865000 & -0.49887000 \\ \mathrm{C} & -4.22286400 & 0.17675200 & -1.75073700 \\ \mathrm{C} & -3.66561100 & 1.57787200 & 0.13902300 \\ \mathrm{C} & -4.83174600 & 1.26219200 & -2.36747600 \\ \mathrm{H} & -4.19858900 & -0.79593700 & -2.22742500 \\ \mathrm{C} & -4.26368500 & 2.66225700 & -0.49131600 \\ \mathrm{H} & -3.23739200 & 1.67713300 & 1.13100800 \\ \mathrm{C} & -4.84653900 & 2.50419000 & -1.74337300 \\ \mathrm{H} & -5.32212000 & 3.34808700 & -2.22849600 \\ \mathrm{H} & -1.42075300 & 0.89740400 & -0.59807400 \\ \mathrm{C} & -4.78758600 & -2.80617500 & 2.59601100 \\ \mathrm{H} & -4.94501500 & -3.77005200 & 3.08769600 \\ \mathrm{H} & -5.75495700 & -2.32220500 & 2.46552900 \\ \mathrm{H} & -4.15372000 & -2.17643200 & 3.23036000 \\ \mathrm{O} & -4.22801600 & -2.97858200 & 1.29934800\end{array}$

$\begin{array}{lrrr}\mathrm{H} & -3.35675700 & -3.37953400 & 1.39427400 \\ \mathrm{C} & 0.42453100 & 0.13319900 & -0.23865400 \\ \mathrm{C} & 1.67348900 & -0.41492700 & -0.39073400 \\ \mathrm{C} & 1.50506400 & 0.93744700 & -0.00112600 \\ \mathrm{~N} & 2.46169300 & -1.42407300 & -0.66745600 \\ \mathrm{~N} & 1.96052600 & 2.11884400 & 0.34699200 \\ \mathrm{C} & 3.91729000 & -1.23151800 & -0.42781500 \\ \mathrm{C} & 1.81081600 & -2.71318100 & -1.04282000 \\ \mathrm{C} & 3.40830700 & 2.38620300 & 0.48362800 \\ \mathrm{C} & 0.98217200 & 3.17044800 & 0.71614400 \\ \mathrm{C} & 4.25341100 & -1.41021200 & 1.05122800 \\ \mathrm{H} & 4.10185900 & -0.19008000 & -0.70196300 \\ \mathrm{C} & 4.81156100 & -2.06422400 & -1.33597200 \\ \mathrm{C} & 2.13394000 & -3.83331400 & -0.06223100 \\ \mathrm{H} & 3.90228200 & 1.47774800 & 0.14003600 \\ \mathrm{C} & 3.84429800 & 3.53455800 & -0.42056300 \\ \mathrm{C} & 3.78492200 & 2.60113500 & 1.94679200 \\ \mathrm{H} & 1.58620000 & 4.01518200 & 1.04944200 \\ \mathrm{C} & 0.17937600 & 3.62270100 & -0.49987500 \\ \mathrm{C} & 0.10572400 & 2.73093400 & 1.88581400\end{array}$




$\begin{array}{lrrrrrrr}\mathrm{H} & 3.61869900 & -0.77841600 & 1.67887300 & \mathrm{H} & 4.86679700 & 2.71680500 & 2.03702100 \\ \mathrm{H} & 4.11726300 & -2.44593500 & 1.36601500 & \mathrm{H} & -0.48608700 & 4.44293300 & -0.22388000 \\ \mathrm{H} & 5.29485500 & -1.13421000 & 1.23240300 & \mathrm{H} & 0.83860300 & 3.96640100 & -1.29885100 \\ \mathrm{H} & 4.73231600 & -3.13324100 & -1.13845100 & \mathrm{H} & -0.44472100 & 2.81779400 & -0.89867100 \\ \mathrm{H} & 4.59870700 & -1.88018400 & -2.38930100 & \mathrm{H} & -0.54895100 & 1.89919800 & 1.61183600 \\ \mathrm{H} & 5.84732600 & -1.77505500 & -1.14730700 & \mathrm{H} & 0.71507100 & 2.41971500 & 2.73639200 \\ \mathrm{H} & 3.17542800 & -4.15563700 & -0.11657700 & \mathrm{H} & -0.52749000 & 3.56173100 & 2.20372600 \\ \mathrm{H} & 1.90498700 & -3.52997500 & 0.96070000 & \mathrm{H} & -5.29514700 & 1.13674700 & -3.33858300 \\ \mathrm{H} & 1.50996200 & -4.69673000 & -0.30310200 & \mathrm{H} & -4.29317900 & 3.62488000 & 0.00520000 \\ \mathrm{H} & 3.38519100 & 4.48154600 & -0.12566600 & \mathrm{H} & 0.74765700 & -2.50373600 & -0.92336800 \\ \mathrm{H} & 4.92621900 & 3.66356600 & -0.35275700 & \mathrm{C} & 2.04426000 & -3.09668600 & -2.50256300 \\ \mathrm{H} & 3.58726500 & 3.33397200 & -1.46270200 & \mathrm{H} & 3.03106700 & -3.52417300 & -2.67832600 \\ \mathrm{H} & 3.32450600 & 3.50413400 & 2.35597700 & \mathrm{H} & 1.30664300 & -3.85187600 & -2.78318600 \\ \mathrm{H} & 3.47894000 & 1.74967900 & 2.55872600 & \mathrm{H} & 1.91049200 & -2.23666100 & -3.16256700\end{array}$

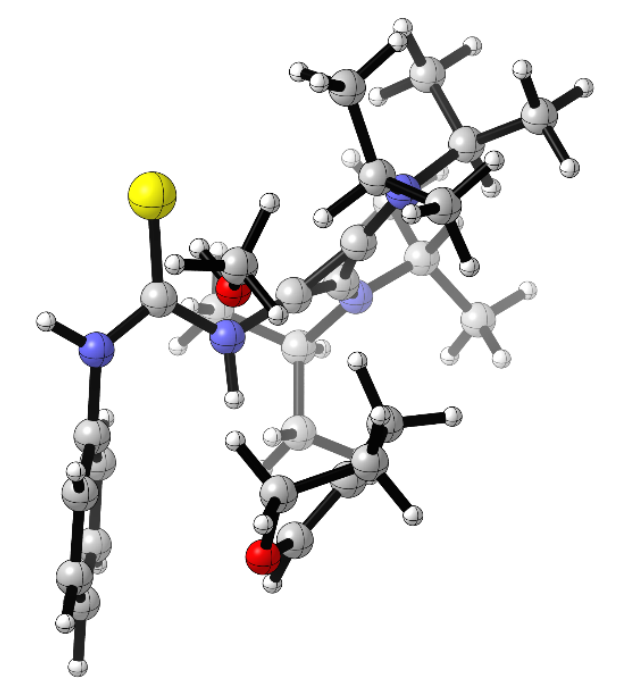

Figure S49. Optimized Structure of E,Z-1-Cy-INT2.

- Thermochemistry -

(0 imaginary frequencies)

Zero-point correction $=$

Thermal correction to Energy=

0.727291 (Hartree/Particle)

Thermal correction to Enthalpy=

0.768940

0.769885

Thermal correction to Gibbs Free Energy $=0.650424$

Sum of electronic and zero-point Energies $=\quad-1861.885849$

Sum of electronic and thermal Energies $=\quad-1861.844199$

Sum of electronic and thermal Enthalpies $=\quad-1861.843255$

Sum of electronic and thermal Free Energies $=\quad-1861.962716$

$\begin{array}{llllllll}\mathrm{C} & -1.09573900 & 0.18933600 & -1.87789700 & \mathrm{C} & -4.45922600 & -0.11795000 & -0.81416100 \\ \mathrm{~S} & -0.05668300 & 0.93416800 & -2.96362900 & \mathrm{C} & -3.11450900 & -2.09393300 & -1.16951400 \\ \mathrm{~N} & -2.40943000 & 0.04555900 & -2.11740900 & \mathrm{C} & -5.36925900 & -0.87514700 & -0.08755900 \\ \mathrm{H} & -2.72967100 & 0.46766900 & -2.97584300 & \mathrm{H} & -4.61020500 & 0.94556700 & -0.95661300 \\ \mathrm{~N} & -0.66769700 & -0.32792700 & -0.68694000 & \mathrm{C} & -4.01711900 & -2.83859800 & -0.42543300 \\ \mathrm{C} & -3.33357100 & -0.72867100 & -1.34915400 & \mathrm{H} & -2.24972300 & -2.56369600 & -1.62441900\end{array}$




\begin{tabular}{|c|c|c|c|c|c|c|c|}
\hline $\mathrm{C}$ & -5.14605200 & -2.23003100 & 0.11500400 & $\mathrm{H}$ & 4.00594400 & -1.58459700 & 0.79568800 \\
\hline $\mathrm{H}$ & -5.85418900 & -2.81587200 & 0.68865800 & $\mathrm{C}$ & 3.64696600 & -3.30973100 & 2.00080100 \\
\hline $\mathrm{H}$ & -1.34350200 & -0.59634100 & 0.02972800 & $\mathrm{C}$ & 4.35985800 & -3.28187000 & -0.44256200 \\
\hline $\mathrm{C}$ & -2.70764200 & -0.37765700 & 2.29218400 & $\mathrm{H}$ & 1.90286800 & -4.38783400 & 0.17070600 \\
\hline $\mathrm{C}$ & -1.38583900 & -0.17128700 & 2.33845700 & $\mathrm{C}$ & 0.08512800 & -3.53824100 & 0.88880800 \\
\hline $\mathrm{C}$ & -0.80297000 & 1.21799400 & 2.34607600 & $\mathrm{C}$ & 0.90778900 & -3.53330200 & -1.51655900 \\
\hline $\mathrm{C}$ & -1.90675000 & 2.23388600 & 2.64033900 & $\mathrm{H}$ & 4.27768700 & -0.06073600 & -1.37267000 \\
\hline $\mathrm{C}$ & -3.14209700 & 1.87855400 & 1.82941300 & $\mathrm{H}$ & 4.74216300 & 1.63119300 & -1.58783500 \\
\hline $\mathrm{H}$ & -0.00990800 & 1.28387500 & 3.09733200 & $\mathrm{H}$ & 5.78046400 & 0.54310900 & -0.65757400 \\
\hline $\mathrm{H}$ & -0.35101400 & 1.46559100 & 1.37927900 & $\mathrm{H}$ & 4.68802500 & 3.23761700 & 0.53477000 \\
\hline $\mathrm{H}$ & -2.15814500 & 2.22253300 & 3.70483000 & $\mathrm{H}$ & 4.15119800 & 2.54461900 & 2.07942600 \\
\hline $\mathrm{H}$ & -1.58522100 & 3.24572700 & 2.38311300 & $\mathrm{H}$ & 5.68727700 & 2.05259800 & 1.36368000 \\
\hline $\mathrm{H}$ & -3.96842700 & 2.56195600 & 2.02093000 & $\mathrm{H}$ & 3.50009900 & 3.69184100 & -1.22090500 \\
\hline $\mathrm{H}$ & -2.89799800 & 1.89719200 & 0.76066700 & $\mathrm{H}$ & 2.50651700 & 2.63372000 & -2.25134600 \\
\hline $\mathrm{O}$ & -3.64022200 & 0.57897700 & 2.16306100 & $\mathrm{H}$ & 1.86908600 & 4.17534400 & -1.67359500 \\
\hline $\mathrm{H}$ & -0.74365400 & -1.03295600 & 2.48439600 & $\mathrm{H}$ & 3.23264900 & -4.31887700 & 1.93222700 \\
\hline $\mathrm{C}$ & -1.56866600 & 4.42190200 & -0.69752800 & $\mathrm{H}$ & 4.68796000 & -3.40551500 & 2.31542700 \\
\hline $\mathrm{H}$ & -0.65154400 & 4.98760000 & -0.90234100 & $\mathrm{H}$ & 3.10246500 & -2.76400900 & 2.77431300 \\
\hline $\mathrm{H}$ & -1.92300300 & 4.68973600 & 0.29892400 & $\mathrm{H}$ & 3.99166100 & -4.29579000 & -0.61829400 \\
\hline $\mathrm{H}$ & -2.33183500 & 4.71532000 & -1.42575900 & $\mathrm{H}$ & 4.30044200 & -2.72511300 & -1.38024900 \\
\hline $\mathrm{O}$ & -1.34147400 & 3.02461300 & -0.69922000 & $\mathrm{H}$ & 5.41065200 & -3.36115100 & -0.15708400 \\
\hline $\mathrm{H}$ & -1.03844600 & 2.75370100 & -1.57363700 & $\mathrm{H}$ & -0.46808200 & -4.46745000 & 0.73800600 \\
\hline $\mathrm{C}$ & 0.65053600 & -0.35418400 & -0.30914300 & $\mathrm{H}$ & 0.39208400 & -3.48938100 & 1.93569600 \\
\hline $\mathrm{C}$ & 1.82634200 & 0.27268100 & 0.00798100 & $\mathrm{H}$ & -0.60144500 & -2.71255300 & 0.68596800 \\
\hline $\mathrm{C}$ & 1.70071300 & -1.14680000 & 0.05736900 & $\mathrm{H}$ & 0.29804000 & -2.66603600 & -1.78472500 \\
\hline $\mathrm{N}$ & 2.55178900 & 1.35045100 & 0.14442200 & $\mathrm{H}$ & 1.79231500 & -3.53933300 & -2.15610100 \\
\hline $\mathrm{N}$ & 2.17537500 & -2.34953500 & 0.25804800 & $\mathrm{H}$ & 0.32622700 & -4.43255300 & -1.73037900 \\
\hline $\mathrm{C}$ & 4.00520700 & 1.16487800 & 0.40449300 & $\mathrm{H}$ & -6.24324600 & -0.39830800 & 0.33752400 \\
\hline $\mathrm{C}$ & 1.88424000 & 2.64557100 & -0.17693100 & $\mathrm{H}$ & -3.85174400 & -3.90027100 & -0.28499600 \\
\hline $\mathrm{C}$ & 3.57872600 & -2.57886400 & 0.66384200 & $\mathrm{H}$ & 0.86793300 & 2.35397000 & -0.44024600 \\
\hline $\mathrm{C}$ & 1.29458700 & -3.50772200 & -0.04067200 & $\mathrm{C}$ & 1.76644800 & 3.57799200 & 1.02699100 \\
\hline $\mathrm{C}$ & 4.74237200 & 0.79905500 & -0.88194800 & $\mathrm{H}$ & 2.68741500 & 4.12070100 & 1.23878400 \\
\hline $\mathrm{H}$ & 4.05203900 & 0.31430100 & 1.08945200 & $\mathrm{H}$ & 0.98951200 & 4.31460000 & 0.81125000 \\
\hline $\mathrm{C}$ & 4.65575200 & 2.32937900 & 1.13735300 & $\mathrm{H}$ & 1.46439300 & 3.03068900 & 1.92194100 \\
\hline $\mathrm{C}$ & 2.49013300 & 3.31831100 & -1.40196700 & $\mathrm{H}$ & -3.15803000 & -1.36094300 & 2.37387800 \\
\hline
\end{tabular}

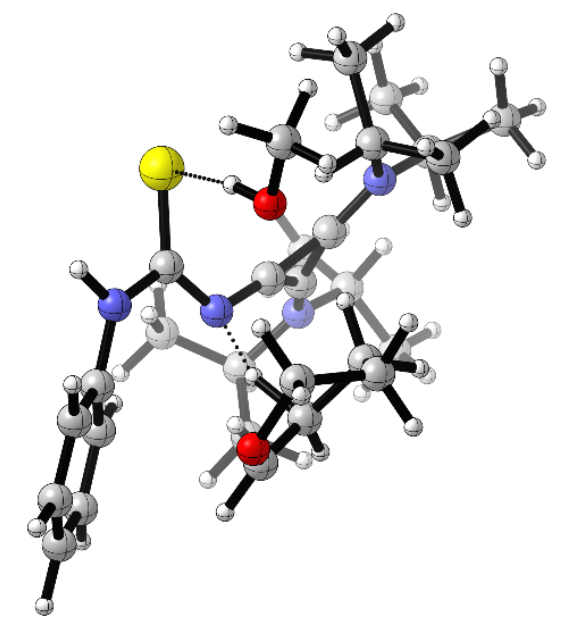

Figure S50. Optimized Structure of E,Z-1-Cy-TS 1 .

- Thermochemistry - 
$(1$ imaginary frequency $=-664.73 \mathrm{~Hz})$

Zero-point correction=

Thermal correction to Energy=

Thermal correction to Enthalpy=

Thermal correction to Gibbs Free Energy=

Sum of electronic and zero-point Energies=

Sum of electronic and thermal Energies=

Sum of electronic and thermal Enthalpies=

Sum of electronic and thermal Free Energies=
0.723417 (Hartree/Particle)

0.763687

0.764632

0.650510

$-1861.859816$

$-1861.819545$

$-1861.818601$

$-1861.932723$

$\begin{array}{lrrr}\mathrm{C} & -1.12351300 & 0.11873200 & -1.60401000 \\ \mathrm{~S} & -0.25338100 & 1.04600800 & -2.73090900 \\ \mathrm{~N} & -2.46720100 & -0.02447100 & -1.77882600 \\ \mathrm{H} & -2.82967500 & 0.49545900 & -2.56312500 \\ \mathrm{~N} & -0.60961000 & -0.49989700 & -0.53067700 \\ \mathrm{C} & -3.35686200 & -0.92625300 & -1.14429300 \\ \mathrm{C} & -4.66850600 & -0.50927000 & -0.92556200 \\ \mathrm{C} & -2.97548000 & -2.21956200 & -0.78253100 \\ \mathrm{C} & -5.58438600 & -1.36535200 & -0.32846300 \\ \mathrm{H} & -4.96510300 & 0.49318000 & -1.21447500 \\ \mathrm{C} & -3.89207000 & -3.06213200 & -0.16938300 \\ \mathrm{H} & -1.96945200 & -2.55884900 & -0.98461500 \\ \mathrm{C} & -5.19729400 & -2.64016800 & 0.06674000 \\ \mathrm{H} & -5.91129200 & -3.30767000 & 0.53378900 \\ \mathrm{H} & -1.37580500 & -0.39760900 & 0.75295000 \\ \mathrm{C} & -3.06166000 & -0.15238800 & 1.91740500 \\ \mathrm{C} & -1.66036100 & -0.09776100 & 1.94132600 \\ \mathrm{C} & -0.99940100 & 1.26102300 & 2.18410300 \\ \mathrm{C} & -2.03995400 & 2.29469700 & 2.60844400 \\ \mathrm{C} & -3.25322400 & 2.19274400 & 1.71039700 \\ \mathrm{H} & -0.22256900 & 1.16142300 & 2.94402800 \\ \mathrm{H} & -0.52449500 & 1.61102400 & 1.26441300 \\ \mathrm{H} & -2.33933100 & 2.14441300 & 3.65045900 \\ \mathrm{H} & -1.63505600 & 3.30404200 & 2.51771300 \\ \mathrm{H} & -4.06725000 & 2.85092100 & 2.00302300 \\ \mathrm{H} & -2.98068800 & 2.36058500 & 0.66536300 \\ \mathrm{O} & -3.84020100 & 0.85289000 & 1.78773500 \\ \mathrm{H} & -1.22910700 & -0.96911800 & 2.43497500 \\ \mathrm{C} & -1.42850700 & 4.58281700 & -0.94819600 \\ \mathrm{H} & -0.43877000 & 4.94742300 & -1.24792500 \\ \mathrm{H} & -1.74587800 & 5.14215900 & -0.06639600 \\ \mathrm{H} & -2.13538600 & 4.78471800 & -1.75971100 \\ \mathrm{O} & -1.40207800 & 3.21146600 & -0.59765700 \\ \mathrm{H} & -1.11425900 & 2.70005800 & -1.37109400 \\ \mathrm{C} & 0.72023500 & -0.42301700 & -0.27938900 \\ \mathrm{C} & 1.88589400 & 0.25455300 & 0.01055400 \\ \mathrm{C} & 1.83023300 & -1.15347100 & 0.06188800 \\ \mathrm{~N} & 2.57318000 & 1.36764300 & 0.15830700 \\ \mathrm{~N} & 2.36514200 & -2.34211900 & 0.24824800 \\ \mathrm{C} & 4.02902600 & 1.22775000 & 0.41289000 \\ \mathrm{C} & 1.87443000 & 2.63489500 & -0.18174400\end{array}$

$\begin{array}{lrrr}\mathrm{C} & 3.79842300 & -2.51617100 & 0.55008600 \\ \mathrm{C} & 1.50799600 & -3.52784800 & 0.01592600 \\ \mathrm{C} & 4.78334700 & 0.88269800 & -0.87049900 \\ \mathrm{H} & 4.10420500 & 0.37950700 & 1.09917700 \\ \mathrm{C} & 4.64543600 & 2.41188500 & 1.14521400 \\ \mathrm{C} & 2.46658900 & 3.31562000 & -1.40977100 \\ \mathrm{H} & 4.20149500 & -1.50682400 & 0.63370500 \\ \mathrm{C} & 3.99569100 & -3.21845400 & 1.89044700 \\ \mathrm{C} & 4.52494500 & -3.21275300 & -0.59752200 \\ \mathrm{H} & 2.14623200 & -4.38845200 & 0.22139900 \\ \mathrm{C} & 0.34060700 & -3.56026600 & 0.99963400 \\ \mathrm{C} & 1.04988600 & -3.60174100 & -1.43835500 \\ \mathrm{H} & 4.33421800 & 0.02101200 & -1.37198300 \\ \mathrm{H} & 4.77815600 & 1.71969000 & -1.57052600 \\ \mathrm{H} & 5.82338700 & 0.63890600 & -0.64002700 \\ \mathrm{H} & 4.65141600 & 3.32037800 & 0.54186800 \\ \mathrm{H} & 4.13245300 & 2.61417700 & 2.08599700 \\ \mathrm{H} & 5.68463000 & 2.16732500 & 1.37405200 \\ \mathrm{H} & 3.46536400 & 3.71849500 & -1.22897300 \\ \mathrm{H} & 2.50386000 & 2.62138900 & -2.25069300 \\ \mathrm{H} & 1.82291200 & 4.15112600 & -1.69533600 \\ \mathrm{H} & 3.61123100 & -4.24152400 & 1.87085000 \\ \mathrm{H} & 5.06024900 & -3.27479600 & 2.12670900 \\ \mathrm{H} & 3.49225700 & -2.67615800 & 2.69384300 \\ \mathrm{H} & 4.18086100 & -4.24175600 & -0.73024500 \\ \mathrm{H} & 4.37689100 & -2.67616700 & -1.53721100 \\ \mathrm{H} & 5.59610400 & -3.25006800 & -0.38865200 \\ \mathrm{H} & -0.20561700 & -4.50003600 & 0.89271500 \\ \mathrm{H} & 0.69710600 & -3.48597200 & 2.02966800 \\ \mathrm{H} & -0.35887000 & -2.74353000 & 0.80195300 \\ \mathrm{H} & 0.40818100 & -2.75436000 & -1.69484300 \\ \mathrm{H} & 1.90301000 & -3.60429900 & -2.11933800 \\ \mathrm{H} & 0.48019600 & -4.51916800 & -1.60245100 \\ \mathrm{H} & -6.60156600 & -1.02960300 & -0.16596300 \\ \mathrm{H} & -3.59000800 & -4.06688800 & 0.10359000 \\ \mathrm{H} & 0.87520500 & 2.30708200 & -0.46463800 \\ \mathrm{C} & 1.71035800 & 3.57775800 & 1.00911400 \\ \mathrm{H} & 2.61126400 & 4.15022900 & 1.23014800 \\ \mathrm{H} & 0.91343600 & 4.28723000 & 0.77577600 \\ \mathrm{H} & 1.41727900 & 3.02813900 & 1.90668300 \\ \mathrm{H} & -3.60094400 & -1.09770200 & 1.94122000\end{array}$




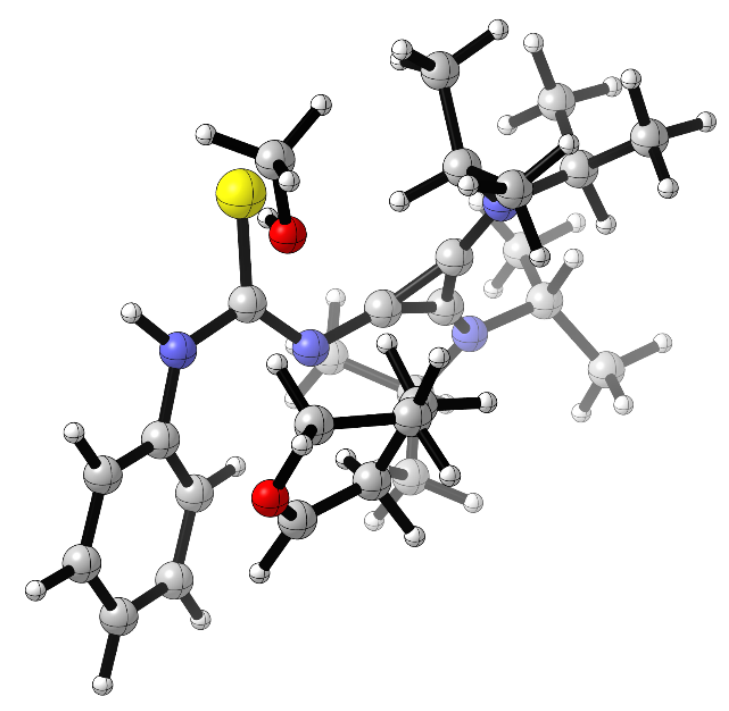

Figure S51. Optimized Structure of E,Z-1-Cy-INT . $_{3}$

- Thermochemistry -

(0 imaginary frequencies)

Zero-point correction=

Thermal correction to Energy=

0.726499 (Hartree/Particle)

Thermal correction to Enthalpy=

0.767764

0.768709

Thermal correction to Gibbs Free Energy=

Sum of electronic and zero-point Energies=

0.651185

Sum of electronic and thermal Energies=

$-1861.860769$

Sum of electronic and thermal Enthalpies=

$-1861.819504$

Sum of electronic and thermal Free Energies=

$-1861.818560$

$-1861.936083$

$\begin{array}{lrrr}\mathrm{C} & -1.05863800 & -0.14875900 & -1.58291900 \\ \mathrm{~S} & -0.40145500 & 1.00240000 & -2.66632000 \\ \mathrm{~N} & -2.40027100 & -0.41653200 & -1.72086200 \\ \mathrm{H} & -2.83417900 & 0.12654500 & -2.45108700 \\ \mathrm{~N} & -0.41419000 & -0.81574900 & -0.63824200 \\ \mathrm{C} & -3.22572900 & -1.34017100 & -1.05933500 \\ \mathrm{C} & -4.59754100 & -1.06107400 & -1.01653400 \\ \mathrm{C} & -2.75720700 & -2.52414100 & -0.47889900 \\ \mathrm{C} & -5.47747800 & -1.93004100 & -0.38717600 \\ \mathrm{H} & -4.97212400 & -0.15516200 & -1.48203300 \\ \mathrm{C} & -3.64509600 & -3.37604600 & 0.16599300 \\ \mathrm{H} & -1.70649500 & -2.76351700 & -0.53899200 \\ \mathrm{C} & -5.00486700 & -3.08622000 & 0.22529600 \\ \mathrm{H} & -5.69027100 & -3.76551100 & 0.71720100 \\ \mathrm{H} & -1.52486400 & -0.81661800 & 1.20901700 \\ \mathrm{C} & -3.38786100 & -0.17496900 & 1.79336100 \\ \mathrm{C} & -1.94376100 & -0.17196500 & 2.00476400 \\ \mathrm{C} & -1.29201100 & 1.20691100 & 1.99165500 \\ \mathrm{C} & -2.27059200 & 2.26651000 & 2.48872100 \\ \mathrm{C} & -3.52707200 & 2.21370900 & 1.65587000 \\ \mathrm{H} & -0.38546600 & 1.18934400 & 2.59697700\end{array}$

$\begin{array}{lrrr}\mathrm{H} & -1.00107500 & 1.45628000 & 0.96904200 \\ \mathrm{H} & -2.50538000 & 2.12674000 & 3.54908800 \\ \mathrm{H} & -1.84415100 & 3.26427800 & 2.36968300 \\ \mathrm{H} & -4.34795200 & 2.81532300 & 2.03647600 \\ \mathrm{H} & -3.31818200 & 2.43821800 & 0.60626000 \\ \mathrm{O} & -4.10546100 & 0.84024000 & 1.65013500 \\ \mathrm{H} & -1.78591500 & -0.73970200 & 2.93343900 \\ \mathrm{C} & -1.94814700 & 4.38979200 & -1.28844200 \\ \mathrm{H} & -0.95283700 & 4.83487400 & -1.40373600 \\ \mathrm{H} & -2.52230400 & 5.01111500 & -0.59860900 \\ \mathrm{H} & -2.44938500 & 4.39790500 & -2.26137700 \\ \mathrm{O} & -1.87990300 & 3.08417700 & -0.74342900 \\ \mathrm{H} & -1.47415500 & 2.49934100 & -1.41112200 \\ \mathrm{C} & 0.85864300 & -0.51383200 & -0.34656100 \\ \mathrm{C} & 1.89239600 & 0.33943200 & -0.00255500 \\ \mathrm{C} & 2.07195000 & -1.04872200 & 0.02244600 \\ \mathrm{~N} & 2.38370900 & 1.55206100 & 0.20735100 \\ \mathrm{~N} & 2.79103500 & -2.14135000 & 0.21077600 \\ \mathrm{C} & 3.82635200 & 1.63382800 & 0.53586300 \\ \mathrm{C} & 1.52632300 & 2.70135900 & -0.17113200 \\ \mathrm{C} & 4.22761700 & -2.08124200 & 0.52920400\end{array}$




$\begin{array}{lrrrrrrr}\mathrm{C} & 2.13449100 & -3.44675800 & -0.02208600 & \mathrm{H} & 4.34197700 & -3.85712000 & 1.79061800 \\ \mathrm{C} & 4.69753800 & 1.42918500 & -0.70381200 & \mathrm{H} & 5.58546600 & -2.65047300 & 2.10587700 \\ \mathrm{H} & 3.99794300 & 0.79720000 & 1.21997100 & \mathrm{H} & 3.92618100 & -2.36893100 & 2.65983700 \\ \mathrm{C} & 4.21226200 & 2.88834800 & 1.30849100 & \mathrm{H} & 4.90642600 & -3.67475200 & -0.79655500 \\ \mathrm{C} & 2.06535100 & 3.46576600 & -1.37461500 & \mathrm{H} & 4.84380500 & -2.07355900 & -1.55255100 \\ \mathrm{H} & 4.45219500 & -1.02158900 & 0.65013600 & \mathrm{H} & 6.13401000 & -2.47083000 & -0.40636600 \\ \mathrm{C} & 4.53196600 & -2.78235200 & 1.85043000 & \mathrm{H} & 0.58572500 & -4.66848700 & 0.86204500 \\ \mathrm{C} & 5.07332400 & -2.60756100 & -0.62780500 & \mathrm{H} & 1.40473900 & -3.60162000 & 2.01653800 \\ \mathrm{H} & 2.91026600 & -4.19534800 & 0.14775800 & \mathrm{H} & 0.22297000 & -2.93722300 & 0.86442900 \\ \mathrm{C} & 1.01981300 & -3.67509900 & 0.99680600 & \mathrm{H} & 0.85212200 & -2.86144900 & -1.67902700 \\ \mathrm{C} & 1.64696900 & -3.57985000 & -1.46264900 & \mathrm{H} & 2.46374700 & -3.41703600 & -2.16861200 \\ \mathrm{H} & 4.40425900 & 0.52687400 & -1.24722300 & \mathrm{H} & 1.24734700 & -4.58304900 & -1.62738600 \\ \mathrm{H} & 4.61956200 & 2.27419100 & -1.38987000 & \mathrm{H} & -6.53634400 & -1.69987700 & -0.37406200 \\ \mathrm{H} & 5.74585900 & 1.32360200 & -0.41270900 & \mathrm{H} & -3.26771800 & -4.29106800 & 0.60840600 \\ \mathrm{H} & 4.12381000 & 3.79379000 & 0.70673700 & \mathrm{H} & 0.60438600 & 2.22951800 & -0.50799300 \\ \mathrm{H} & 3.61413600 & 3.00405900 & 2.21302400 & \mathrm{C} & 1.16654500 & 3.61462400 & 1.00120000 \\ \mathrm{H} & 5.25888700 & 2.79711700 & 1.60598600 & \mathrm{H} & 1.93890600 & 4.35160000 & 1.22311300 \\ \mathrm{H} & 2.98801300 & 4.00589400 & -1.15107200 & \mathrm{H} & 0.25098300 & 4.15321600 & 0.74420300 \\ \mathrm{H} & 2.23768900 & 2.78560900 & -2.21024500 & \mathrm{H} & 0.97468000 & 3.03474800 & 1.90698300 \\ \mathrm{H} & 1.32149900 & 4.20078100 & -1.69126400 & \mathrm{H} & -3.94782100 & -1.11196200 & 1.76987000\end{array}$

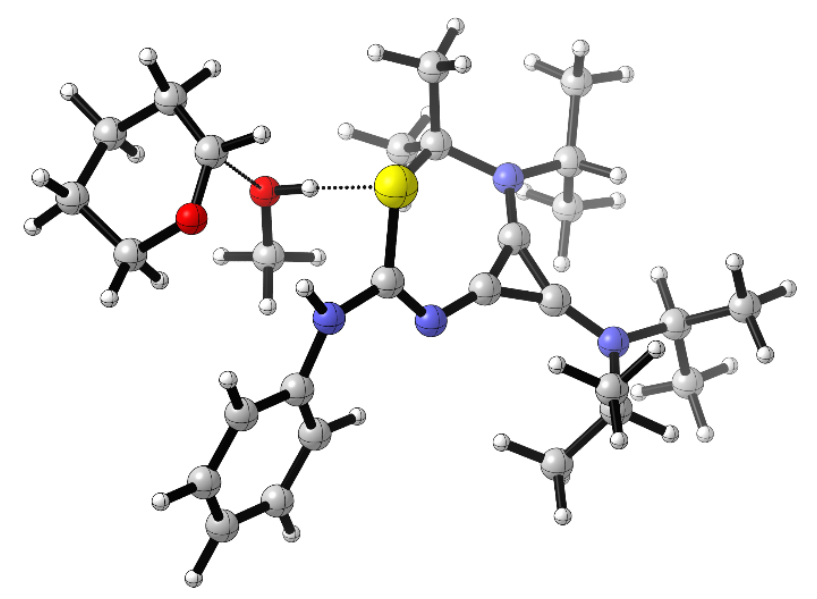

Figure S52. Optimized Structure of E,Z-1-Cy-TS 2 .

- Thermochemistry -

$(1$ imaginary frequency $=-65.27 \mathrm{~Hz})$

Zero-point correction $=$

Thermal correction to Energy=

Thermal correction to Enthalpy=

Thermal correction to Gibbs Free Energy=

Sum of electronic and zero-point Energies=

Sum of electronic and thermal Energies $=$

Sum of electronic and thermal Enthalpies=

Sum of electronic and thermal Free Energies=
0.728155 (Hartree/Particle)

$$
0.768097
$$

0.769042

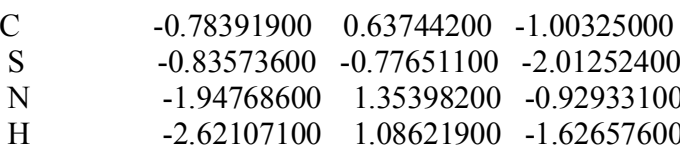

0.654409

$-1861.854060$

$-1861.814118$

$-1861.813173$

$-1861.927806$

$\begin{array}{lrrr}\mathrm{N} & 0.24673600 & 1.07664000 & -0.32467100 \\ \mathrm{C} & -2.35669400 & 2.39129300 & -0.07443800 \\ \mathrm{C} & -1.66804800 & 2.77760200 & 1.07718600 \\ \mathrm{C} & -3.56965500 & 3.01787300 & -0.38548900\end{array}$




\begin{tabular}{|c|c|c|c|c|c|c|c|}
\hline $\mathrm{C}$ & -2.20067200 & 3.76652500 & 1.89579100 & $\mathrm{C}$ & 0.96769100 & -3.30187100 & 1.87307400 \\
\hline $\mathrm{H}$ & -0.72112900 & 2.31725500 & 1.31227400 & $\mathrm{H}$ & 5.07705200 & -0.20658700 & 0.14672900 \\
\hline $\mathrm{C}$ & -4.08788300 & 4.00245800 & 0.44092400 & $\mathrm{C}$ & 5.99885700 & 0.99468600 & -1.35732400 \\
\hline $\mathrm{H}$ & -4.09458500 & 2.74389500 & -1.29646400 & $\mathrm{C}$ & 5.83953100 & 1.52169200 & 1.12517400 \\
\hline $\mathrm{C}$ & -3.40697300 & 4.38172300 & 1.59229200 & $\mathrm{H}$ & 4.54123400 & 3.23465100 & -0.60383100 \\
\hline $\mathrm{H}$ & -3.80730300 & 5.15446300 & 2.23691500 & $\mathrm{C}$ & 2.89479700 & 2.94259600 & -1.93461500 \\
\hline $\mathrm{H}$ & -3.64523800 & -3.46896100 & -0.81468200 & $\mathrm{C}$ & 2.75091100 & 3.43521800 & 0.54997100 \\
\hline $\mathrm{C}$ & -4.04963600 & -1.53784400 & -1.44951000 & $\mathrm{H}$ & 4.00952600 & -1.18509200 & 2.07057100 \\
\hline $\mathrm{C}$ & -4.51196800 & -2.80523400 & -0.87000400 & $\mathrm{H}$ & 3.63768700 & -2.84589900 & 2.55524300 \\
\hline $\mathrm{C}$ & -5.25013300 & -2.66714100 & 0.45653100 & $\mathrm{H}$ & 5.23112700 & -2.45338200 & 1.90067600 \\
\hline $\mathrm{C}$ & -6.06145700 & -1.37221300 & 0.46653900 & $\mathrm{H}$ & 3.29996100 & -4.67028900 & 0.69396800 \\
\hline $\mathrm{C}$ & -5.16893100 & -0.19038200 & 0.17444800 & $\mathrm{H}$ & 3.54728100 & -4.19711300 & -0.99990300 \\
\hline $\mathrm{H}$ & -5.89908300 & -3.52912500 & 0.61124100 & $\mathrm{H}$ & 4.91086600 & -4.25584900 & 0.12243500 \\
\hline $\mathrm{H}$ & -4.50853100 & -2.65156400 & 1.25599600 & $\mathrm{H}$ & 1.66475200 & -4.12186500 & 2.05824200 \\
\hline $\mathrm{H}$ & -6.86871900 & -1.41390100 & -0.27184000 & $\mathrm{H}$ & 1.16503500 & -2.51074100 & 2.60059300 \\
\hline $\mathrm{H}$ & -6.52693900 & -1.20787500 & 1.44035200 & $\mathrm{H}$ & -0.04196900 & -3.68185800 & 2.04729100 \\
\hline $\mathrm{H}$ & -5.69803900 & 0.74182200 & -0.00376600 & $\mathrm{H}$ & 6.14820900 & 2.04367700 & -1.62619300 \\
\hline $\mathrm{H}$ & -4.39410100 & -0.03437300 & 0.92494300 & $\mathrm{H}$ & 6.98551100 & 0.54742200 & -1.21784700 \\
\hline $\mathrm{O}$ & -4.40519400 & -0.39286400 & -1.07684500 & $\mathrm{H}$ & 5.50640900 & 0.49244400 & -2.19290000 \\
\hline $\mathrm{H}$ & -5.14976800 & -3.22948200 & -1.66449100 & $\mathrm{H}$ & 5.98165200 & 2.59379500 & 0.96695300 \\
\hline $\mathrm{C}$ & -1.95058400 & -0.95326500 & 1.75605300 & $\mathrm{H}$ & 5.23925700 & 1.38313600 & 2.02712700 \\
\hline $\mathrm{H}$ & -2.55342300 & -1.28093500 & 2.60510500 & $\mathrm{H}$ & 6.82423500 & 1.08125300 & 1.29630800 \\
\hline $\mathrm{H}$ & -2.13075500 & 0.11288600 & 1.57979900 & $\mathrm{H}$ & 2.80271600 & 4.00480200 & -2.17126000 \\
\hline $\mathrm{H}$ & -0.89550500 & -1.08405000 & 2.01774700 & $\mathrm{H}$ & 3.48872400 & 2.46452100 & -2.71655500 \\
\hline $\mathrm{O}$ & -2.31326900 & -1.74918200 & 0.63901600 & $\mathrm{H}$ & 1.88988400 & 2.51255000 & -1.94163400 \\
\hline $\mathrm{C}$ & 1.39387800 & 0.39113500 & -0.27034600 & $\mathrm{H}$ & 1.73948300 & 3.02232200 & 0.59600200 \\
\hline $\mathrm{C}$ & 2.17669700 & -0.72765800 & -0.05878500 & $\mathrm{H}$ & 3.23974000 & 3.29493900 & 1.51681600 \\
\hline $\mathrm{C}$ & 2.76390200 & 0.53180100 & -0.20974500 & $\mathrm{H}$ & 2.66441200 & 4.50745700 & 0.36056700 \\
\hline $\mathrm{N}$ & 2.30327900 & -2.02743400 & 0.17304800 & $\mathrm{H}$ & -1.65451700 & 4.05974500 & 2.78517000 \\
\hline $\mathrm{N}$ & 3.80212800 & 1.34406100 & -0.27002000 & $\mathrm{H}$ & -5.02219500 & 4.48426200 & 0.17630700 \\
\hline $\mathrm{C}$ & 3.67434900 & -2.53749000 & 0.39774500 & $\mathrm{H}$ & 0.28413600 & -2.01381500 & 0.32805200 \\
\hline $\mathrm{C}$ & 1.05401500 & -2.77486400 & 0.44316300 & $\mathrm{C}$ & 0.73788300 & -3.84239100 & -0.60121700 \\
\hline $\mathrm{C}$ & 5.17732600 & 0.85528200 & -0.07803300 & $\mathrm{H}$ & 0.89156900 & -3.44535100 & -1.60543400 \\
\hline $\mathrm{C}$ & 3.55269600 & 2.77334800 & -0.56785600 & $\mathrm{H}$ & 1.32616000 & -4.75155700 & -0.47516800 \\
\hline $\mathrm{C}$ & 4.16291100 & -2.23773900 & 1.81662800 & $\mathrm{H}$ & -0.31710400 & -4.11293200 & -0.50256100 \\
\hline $\mathrm{H}$ & 4.29277500 & -1.97214900 & -0.30499700 & $\mathrm{H}$ & -3.40457100 & -1.51653300 & -2.32835100 \\
\hline $\mathrm{C}$ & 3.85237500 & -4.00446800 & 0.02908600 & $\mathrm{H}$ & -1.73418500 & -1.52110700 & -0.11310100 \\
\hline
\end{tabular}




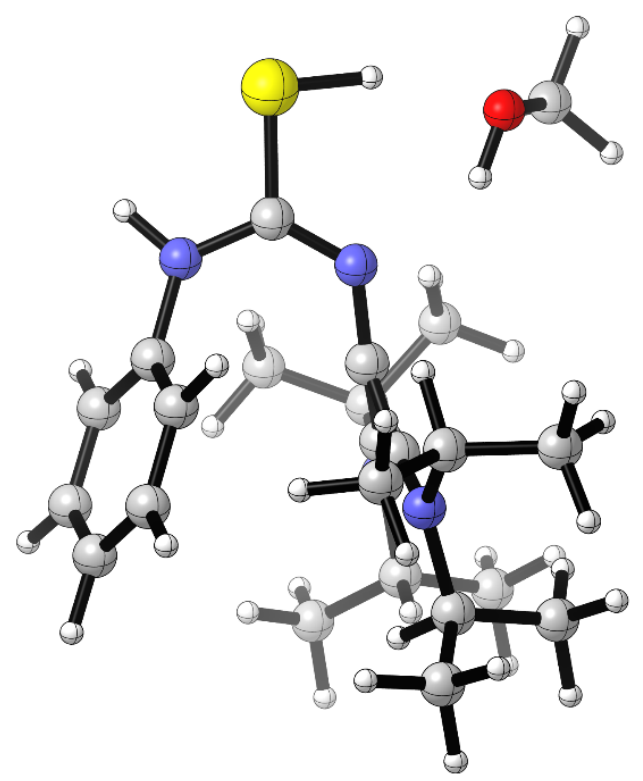

Figure S53. Optimized Structure of E,Z-1-Cy-INT 4 .

- Thermochemistry -

(0 imaginary frequencies)

Zero-point correction $=$

Thermal correction to Energy=

Thermal correction to Enthalpy=

Thermal correction to Gibbs Free Energy=

Sum of electronic and zero-point Energies=

Sum of electronic and thermal Energies=

Sum of electronic and thermal Enthalpies=

Sum of electronic and thermal Free Energies=

\subsection{0 (Hartree/Particle)}

0.634038

0.634982

$\begin{array}{lrrr}\mathrm{C} & 2.53445600 & -0.74138000 & 0.84437900 \\ \mathrm{~S} & 4.19314500 & -1.32232300 & 0.74619700 \\ \mathrm{~N} & 2.23119000 & -0.20876500 & 2.05616300 \\ \mathrm{H} & 3.00373400 & -0.08893100 & 2.69465400 \\ \mathrm{~N} & 1.74525300 & -0.85919100 & -0.17754000 \\ \mathrm{C} & 0.93081900 & 0.04247900 & 2.58655700 \\ \mathrm{C} & 0.65801700 & 1.29039400 & 3.13711400 \\ \mathrm{C} & -0.03643900 & -0.95784800 & 2.60097900 \\ \mathrm{C} & -0.59619000 & 1.54877900 & 3.67293200 \\ \mathrm{H} & 1.42770900 & 2.05275700 & 3.14411100 \\ \mathrm{C} & -1.29449200 & -0.68547100 & 3.12065800 \\ \mathrm{H} & 0.20042200 & -1.94100500 & 2.20905900 \\ \mathrm{C} & -1.57942000 & 0.56665200 & 3.65228300 \\ \mathrm{H} & -2.55702200 & 0.76747000 & 4.07366900 \\ \mathrm{O} & 3.46061900 & -1.81246000 & 2.30156500 \\ \mathrm{H} & 4.14034500 & -1.59388300 & -0.59302800 \\ \mathrm{C} & 0.51430800 & -0.30648000 & -0.2643100 \\ \mathrm{C} & -0.82876300 & -0.52127100 & -0.4378900\end{array}$

$\begin{array}{lrrl}\mathrm{C} & -0.29103100 & 0.76788700 & -0.57695400 \\ \mathrm{~N} & -1.83642400 & -1.37268600 & -0.4751000 \\ \mathrm{~N} & -0.40883400 & 2.04515700 & -0.86532600 \\ \mathrm{C} & -3.17770200 & -0.82319000 & -0.76680400 \\ \mathrm{C} & -1.45239400 & -2.81295300 & -0.46786700 \\ \mathrm{C} & -1.73594400 & 2.66361500 & -1.05716600 \\ \mathrm{C} & 0.81829600 & 2.86182300 & -1.01291100 \\ \mathrm{C} & -3.32795600 & -0.52230700 & -2.25818000 \\ \mathrm{H} & -3.21505700 & 0.12201500 & -0.21687300 \\ \mathrm{C} & -4.32442300 & -1.67035600 & -0.23402200 \\ \mathrm{C} & -1.72496500 & -3.46336200 & -1.82184300 \\ \mathrm{H} & -2.45383400 & 1.85642400 & -0.90710500 \\ \mathrm{C} & -1.98952000 & 3.72738700 & 0.00747200 \\ \mathrm{C} & -1.91025700 & 3.18762500 & -2.47952800 \\ \mathrm{H} & 0.46861100 & 3.83722800 & -1.35376500 \\ \mathrm{C} & 1.53548600 & 3.04894700 & 0.31831200 \\ \mathrm{C} & 1.73445500 & 2.28050300 & -2.08600100 \\ \mathrm{H} & -2.49474200 & 0.08268200 & -2.62623200\end{array}$




$\begin{array}{rrrrrrrr}\mathrm{H} & -3.36943000 & -1.43929100 & -2.84787400 & \mathrm{H} & 1.94609600 & 2.10183500 & 0.67723400 \\ \mathrm{H} & -4.25208500 & 0.03346600 & -2.43414300 & \mathrm{H} & 2.14290600 & 1.31565700 & -1.77209900 \\ \mathrm{H} & -4.41092900 & -2.62604900 & -0.75343800 & \mathrm{H} & 1.20179800 & 2.14643400 & -3.02987400 \\ \mathrm{H} & -4.22192500 & -1.85446600 & 0.83595400 & \mathrm{H} & 2.57534000 & 2.95498300 & -2.25963600 \\ \mathrm{H} & -5.25772500 & -1.12768700 & -0.39559600 & \mathrm{H} & -0.80229700 & 2.51785100 & 4.11158800 \\ \mathrm{H} & -2.79429800 & -3.57801700 & -2.01144300 & \mathrm{H} & -2.05049400 & -1.46133100 & 3.13541500 \\ \mathrm{H} & -1.28442100 & -2.88411100 & -2.63596600 & \mathrm{H} & -0.36782100 & -2.78683700 & -0.33473900 \\ \mathrm{H} & -1.28033700 & -4.46051200 & -1.83692600 & \mathrm{C} & -2.02008200 & -3.62866300 & 0.69154800 \\ \mathrm{H} & -1.29340500 & 4.56483200 & -0.08839200 & \mathrm{H} & -3.07420400 & -3.86987300 & 0.56123200 \\ \mathrm{H} & -2.99984900 & 4.12764700 & -0.09832600 & \mathrm{H} & -1.47445900 & -4.57300900 & 0.75277000 \\ \mathrm{H} & -1.88592200 & 3.30222400 & 1.00859500 & \mathrm{H} & -1.89633500 & -3.10706400 & 1.64121200 \\ \mathrm{H} & -1.23839000 & 4.02257500 & -2.69337400 & \mathrm{H} & 2.62380500 & -1.55496100 & -1.89354000 \\ \mathrm{H} & -1.72506800 & 2.40040600 & -3.21371600 & \mathrm{C} & 3.83587600 & -0.82744300 & -3.24963000 \\ \mathrm{H} & -2.93168900 & 3.54880700 & -2.61575700 & \mathrm{H} & 4.01241700 & 0.14938700 & -2.78161900 \\ \mathrm{H} & 2.36533500 & 3.74843300 & 0.19741800 & \mathrm{H} & 4.76297400 & -1.16086600 & -3.71416200 \\ \mathrm{H} & 0.85516400 & 3.44321400 & 1.07499200 & \mathrm{H} & 3.07871200 & -0.71539700 & -4.03306100\end{array}$

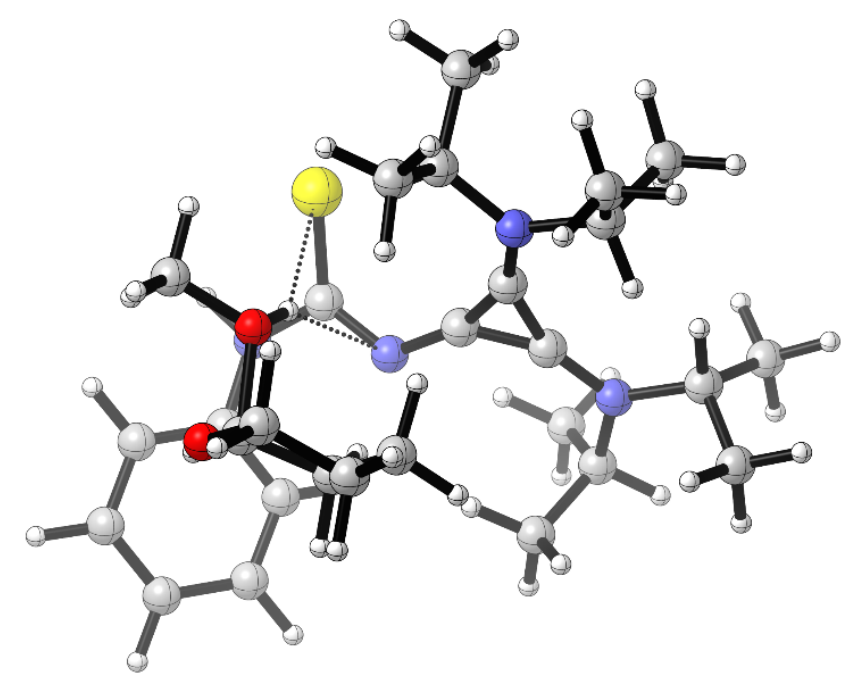

Figure S54. Optimized Structure of E,Z-1-Cy-TS 3 -PA.

- Thermochemistry -

$(1$ imaginary frequency $=-101.65 \mathrm{~Hz})$

Zero-point correction $=$

Thermal correction to Energy=

Thermal correction to Enthalpy=

Thermal correction to Gibbs Free Energy=

Sum of electronic and zero-point Energies=

Sum of electronic and thermal Energies $=$

Sum of electronic and thermal Enthalpies=

Sum of electronic and thermal Free Energies=
0.729627 (Hartree/Particle)

$$
\begin{gathered}
0.768801 \\
0.769745
\end{gathered}
$$

0.657465
-1861.859081
-1861.819908
-1861.818964
-1861.931244

0.657465

$-1861.859081$

$-1861.819908$

$-1861.931244$

$\begin{array}{lccccccc}\mathrm{C} & 1.23662800 & 0.30342800 & -2.06465000 & \mathrm{~N} & 0.53456700 & 0.94400100 & -1.13809000 \\ \mathrm{~S} & 0.70780500 & -0.96197100 & -3.08355500 & \mathrm{C} & 3.39523900 & 1.25069700 & -1.20703900 \\ \mathrm{~N} & 2.55903500 & 0.66783800 & -2.18178400 & \mathrm{C} & 4.68891400 & 0.73369800 & -1.07747600 \\ \mathrm{H} & 3.03052300 & 0.17066200 & -2.92159300 & \mathrm{C} & 2.99315000 & 2.29758800 & -0.37717700\end{array}$




\begin{tabular}{|c|c|c|c|c|c|c|c|}
\hline $\mathrm{C}$ & 5.55203100 & 1.22599300 & -0.10825500 & $\mathrm{C}$ & -3.95738200 & -2.86124300 & 0.93826600 \\
\hline $\mathrm{H}$ & 5.02242300 & -0.05201600 & -1.74941200 & $\mathrm{C}$ & -2.34301900 & -3.20994200 & -2.15581000 \\
\hline $\mathrm{C}$ & 3.85898200 & 2.77012900 & 0.60140700 & $\mathrm{H}$ & -4.20143400 & 1.05579700 & 0.52692200 \\
\hline $\mathrm{H}$ & 2.01255000 & 2.72933700 & -0.50514500 & $\mathrm{C}$ & -4.00830200 & 2.39987100 & 2.17536100 \\
\hline $\mathrm{C}$ & 5.13292500 & 2.23498700 & 0.75122900 & $\mathrm{C}$ & -4.79475800 & 2.96914300 & -0.17612800 \\
\hline $\mathrm{H}$ & 5.80304500 & 2.61755300 & 1.51133500 & $\mathrm{H}$ & -2.48498400 & 4.16978800 & 0.64101900 \\
\hline $\mathrm{H}$ & 1.26513700 & 0.54746900 & 0.96346500 & $\mathrm{C}$ & -0.58752300 & 3.37926000 & 1.22311300 \\
\hline $\mathrm{C}$ & 2.58398500 & -1.08586900 & 1.30389600 & $\mathrm{C}$ & -1.35131900 & 3.87892200 & -1.14690300 \\
\hline $\mathrm{C}$ & 1.56116000 & -0.09836700 & 1.79016400 & $\mathrm{H}$ & -4.43679200 & -0.28775500 & -1.36093900 \\
\hline $\mathrm{C}$ & 0.38702100 & -0.77296700 & 2.49070200 & $\mathrm{H}$ & -4.78206800 & -2.00595800 & -1.57743600 \\
\hline $\mathrm{C}$ & 0.92179800 & -1.78271700 & 3.50533900 & $\mathrm{H}$ & -5.66571000 & -1.07774700 & -0.36134600 \\
\hline $\mathrm{C}$ & 1.86805200 & -2.75919600 & 2.83342100 & $\mathrm{H}$ & -4.01566200 & -3.70654800 & 0.25109800 \\
\hline $\mathrm{H}$ & -0.22860600 & -0.01715600 & 2.98197900 & $\mathrm{H}$ & -3.22362600 & -3.08380400 & 1.71427900 \\
\hline $\mathrm{H}$ & -0.25183700 & -1.27411400 & 1.75620200 & $\mathrm{H}$ & -4.93425400 & -2.76186100 & 1.41563300 \\
\hline $\mathrm{H}$ & 1.44891700 & -1.25962800 & 4.30925700 & $\mathrm{H}$ & -3.23590600 & -3.75356300 & -1.83835200 \\
\hline $\mathrm{H}$ & 0.11068600 & -2.35142100 & 3.96641500 & $\mathrm{H}$ & -2.63242600 & -2.42488200 & -2.85663400 \\
\hline $\mathrm{H}$ & 2.39428000 & -3.38620100 & 3.54925600 & $\mathrm{H}$ & -1.70134500 & -3.91303600 & -2.69223900 \\
\hline $\mathrm{H}$ & 1.33558700 & -3.40255700 & 2.12569500 & $\mathrm{H}$ & -3.71490100 & 3.43013300 & 2.39339600 \\
\hline $\mathrm{O}$ & 2.92837700 & -2.09168100 & 2.10085300 & $\mathrm{H}$ & -5.04207400 & 2.27618100 & 2.50503700 \\
\hline $\mathrm{H}$ & 2.11528700 & 0.53470100 & 2.49280800 & $\mathrm{H}$ & -3.37640500 & 1.73028100 & 2.76412900 \\
\hline $\mathrm{C}$ & 2.78034900 & -2.73788900 & -0.71076800 & $\mathrm{H}$ & -4.57788500 & 4.03171900 & -0.04019000 \\
\hline $\mathrm{H}$ & 2.17747000 & -3.22990200 & -1.46904800 & $\mathrm{H}$ & -4.67763700 & 2.72577400 & -1.23421800 \\
\hline $\mathrm{H}$ & 3.15744900 & -3.44110500 & 0.02594300 & $\mathrm{H}$ & -5.83951500 & 2.81039300 & 0.09945000 \\
\hline $\mathrm{H}$ & 3.59015700 & -2.16944400 & -1.16792900 & $\mathrm{H}$ & -0.10505600 & 4.35688600 & 1.29192200 \\
\hline $\mathrm{O}$ & 1.89958100 & -1.82691400 & 0.00342900 & $\mathrm{H}$ & -0.91831700 & 3.08935800 & 2.22346400 \\
\hline $\mathrm{H}$ & 1.46052800 & -1.23294200 & -0.64302900 & $\mathrm{H}$ & 0.15765000 & 2.66041700 & 0.87221800 \\
\hline $\mathrm{C}$ & -0.70905700 & 0.58797900 & -0.80137300 & $\mathrm{H}$ & -0.60375500 & 3.19981200 & -1.56479600 \\
\hline $\mathrm{C}$ & -1.73903200 & -0.28170300 & -0.46508300 & $\mathrm{H}$ & -2.21255000 & 3.91034100 & -1.81736100 \\
\hline $\mathrm{C}$ & -1.85639800 & 1.08961100 & -0.22210300 & $\mathrm{H}$ & -0.91201800 & 4.87820000 & -1.10748700 \\
\hline $\mathrm{N}$ & -2.26361800 & -1.49603100 & -0.32603200 & $\mathrm{H}$ & 6.55225500 & 0.81729600 & -0.02520400 \\
\hline $\mathrm{N}$ & -2.49327900 & 2.15365600 & 0.23226600 & $\mathrm{H}$ & 3.53626600 & 3.58272200 & 1.24261700 \\
\hline $\mathrm{C}$ & -3.63904200 & -1.55507100 & 0.22265000 & $\mathrm{H}$ & -0.68528800 & -2.15180000 & -1.42349300 \\
\hline $\mathrm{C}$ & -1.56400500 & -2.62389000 & -0.98349500 & $\mathrm{C}$ & -1.06935900 & -3.69633600 & -0.01137900 \\
\hline $\mathrm{C}$ & -3.89344000 & 2.08826000 & 0.68582400 & $\mathrm{H}$ & -1.85355200 & -4.37965700 & 0.31420000 \\
\hline $\mathrm{C}$ & -1.76746700 & 3.44369900 & 0.25540600 & $\mathrm{H}$ & -0.30690000 & -4.29727400 & -0.51501300 \\
\hline $\mathrm{C}$ & -4.68970600 & -1.21233500 & -0.83487600 & $\mathrm{H}$ & -0.61549500 & -3.24468100 & 0.87368100 \\
\hline $\mathrm{H}$ & -3.65670200 & -0.77814600 & 0.99234800 & $\mathrm{H}$ & 3.47445000 & -0.64199900 & 0.85962400 \\
\hline
\end{tabular}

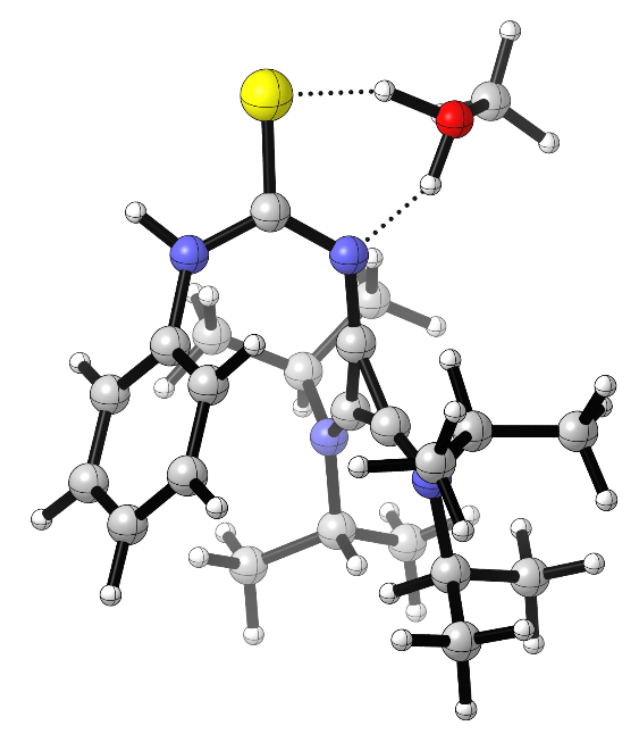

Figure S55. Optimized Structure of E,Z-1-Cy-TS 3 -MA. 
- Thermochemistry -

$(1$ imaginary frequency $=-384.83 \mathrm{~Hz})$

Zero-point correction=

Thermal correction to Energy=

Thermal correction to Enthalpy=

Thermal correction to Gibbs Free Energy=

Sum of electronic and zero-point Energies=

Sum of electronic and thermal Energies=

Sum of electronic and thermal Enthalpies=

Sum of electronic and thermal Free Energies=
0.597671 (Hartree/Particle)

0.630953

0.631898

0.532509

$-1591.443428$

$-1591.410145$

$-1591.409201$

$-1591.508590$

$\begin{array}{lrrr}\mathrm{C} & 2.63573700 & -0.50746800 & 0.74403700 \\ \mathrm{~S} & 4.29636300 & -0.96540800 & 0.62629400 \\ \mathrm{~N} & 2.30975100 & 0.27382700 & 1.80737000 \\ \mathrm{H} & 3.10253300 & 0.55306200 & 2.36703400 \\ \mathrm{~N} & 1.74933300 & -0.91718900 & -0.16139900 \\ \mathrm{C} & 1.02700600 & 0.47464500 & 2.39689800 \\ \mathrm{C} & 0.66059800 & 1.76238600 & 2.77634600 \\ \mathrm{C} & 0.17353200 & -0.59640900 & 2.64975400 \\ \mathrm{C} & -0.57296100 & 1.98525400 & 3.37273500 \\ \mathrm{H} & 1.34702700 & 2.58328800 & 2.60950000 \\ \mathrm{C} & -1.06416100 & -0.36296400 & 3.23372600 \\ \mathrm{H} & 0.48467000 & -1.60356700 & 2.39446800 \\ \mathrm{C} & -1.44546000 & 0.92567200 & 3.58885000 \\ \mathrm{H} & -2.40643800 & 1.09806300 & 4.05825000 \\ \mathrm{O} & 3.29970100 & -2.31357300 & -1.62728400 \\ \mathrm{H} & 3.94118800 & -1.92010200 & -0.79781700 \\ \mathrm{C} & 0.51840700 & -0.36729500 & -0.29338100 \\ \mathrm{C} & -0.83121500 & -0.58873900 & -0.37254300 \\ \mathrm{C} & -0.29983200 & 0.68134500 & -0.66127000 \\ \mathrm{~N} & -1.83744400 & -1.43291000 & -0.25059500 \\ \mathrm{~N} & -0.44334100 & 1.92774200 & -1.05625300 \\ \mathrm{C} & -3.19614700 & -0.91714800 & -0.54948300 \\ \mathrm{C} & -1.45408100 & -2.86643900 & -0.11595900 \\ \mathrm{C} & -1.78560000 & 2.52627000 & -1.20382000 \\ \mathrm{C} & 0.75425900 & 2.70878800 & -1.44831400 \\ \mathrm{C} & -3.43248800 & -0.78085400 & -2.05322600 \\ \mathrm{H} & -3.20354800 & 0.08213300 & -0.10514800 \\ \mathrm{C} & -4.31035700 & -1.69103600 & 0.14100800 \\ \mathrm{C} & -1.82274300 & -3.67882200 & -1.35496100 \\ \mathrm{H} & -2.48047500 & 1.76070200 & -0.85714000 \\ \mathrm{C} & -1.93181500 & 3.74199600 & -0.29363400 \\ \mathrm{C} & -2.11076500 & 2.83313000 & -2.66331900 \\ \mathrm{H} & 0.35697400 & 3.60673500 & -1.92349200 \\ \mathrm{C} & 1.58736100 & 3.13473500 & -0.24658200 \\ & & & \end{array}$

$\begin{array}{lrrr}\mathrm{C} & 1.58143900 & 1.95253100 & -2.48447900 \\ \mathrm{H} & -2.61411400 & -0.23720300 & -2.53271700 \\ \mathrm{H} & -3.52211400 & -1.75640000 & -2.53393600 \\ \mathrm{H} & -4.35766800 & -0.22936500 & -2.23682600 \\ \mathrm{H} & -4.42722900 & -2.69925000 & -0.25963500 \\ \mathrm{H} & -4.14884200 & -1.75098100 & 1.21768800 \\ \mathrm{H} & -5.25097700 & -1.16398500 & -0.03008600 \\ \mathrm{H} & -2.90257500 & -3.80179900 & -1.45895400 \\ \mathrm{H} & -1.43639500 & -3.21189300 & -2.26359600 \\ \mathrm{H} & -1.38609000 & -4.67637300 & -1.27262400 \\ \mathrm{H} & -1.25479300 & 4.54779600 & -0.58976500 \\ \mathrm{H} & -2.95010700 & 4.13150500 & -0.35395000 \\ \mathrm{H} & -1.72199200 & 3.47435200 & 0.74447000 \\ \mathrm{H} & -1.48464300 & 3.63498800 & -3.06222500 \\ \mathrm{H} & -1.97902200 & 1.94880300 & -3.29055100 \\ \mathrm{H} & -3.14946500 & 3.15934600 & -2.74779900 \\ \mathrm{H} & 2.39920200 & 3.78751800 & -0.57465200 \\ \mathrm{H} & 0.97734900 & 3.67973600 & 0.47530700 \\ \mathrm{H} & 2.03232400 & 2.26901600 & 0.24889500 \\ \mathrm{H} & 2.04604200 & 1.06657000 & -2.04169900 \\ \mathrm{H} & 0.96450500 & 1.64436400 & -3.33169500 \\ \mathrm{H} & 2.38156300 & 2.59507500 & -2.85703700 \\ \mathrm{H} & -0.84801900 & 2.98895900 & 3.67515400 \\ \mathrm{H} & -1.72663900 & -1.19481900 & 3.44150700 \\ \mathrm{H} & -0.36296800 & -2.83229200 & -0.06202300 \\ \mathrm{C} & -1.93370400 & -3.52341900 & 1.17590300 \\ \mathrm{H} & -2.99802400 & -3.75476700 & 1.16317000 \\ \mathrm{H} & -1.39597600 & -4.46488700 & 1.30963500 \\ \mathrm{H} & -1.72243200 & -2.89000600 & 2.03780200 \\ \mathrm{H} & 2.45743700 & -1.87747400 & -1.22733500 \\ \mathrm{C} & 3.65543600 & -1.67337300 & -2.86948500 \\ \mathrm{H} & 3.78357700 & -0.59934200 & -2.71833700 \\ \mathrm{H} & 4.58702500 & -2.11889000 & -3.20966800 \\ \mathrm{H} & 2.86694700 & -1.87051600 & -3.59326100\end{array}$




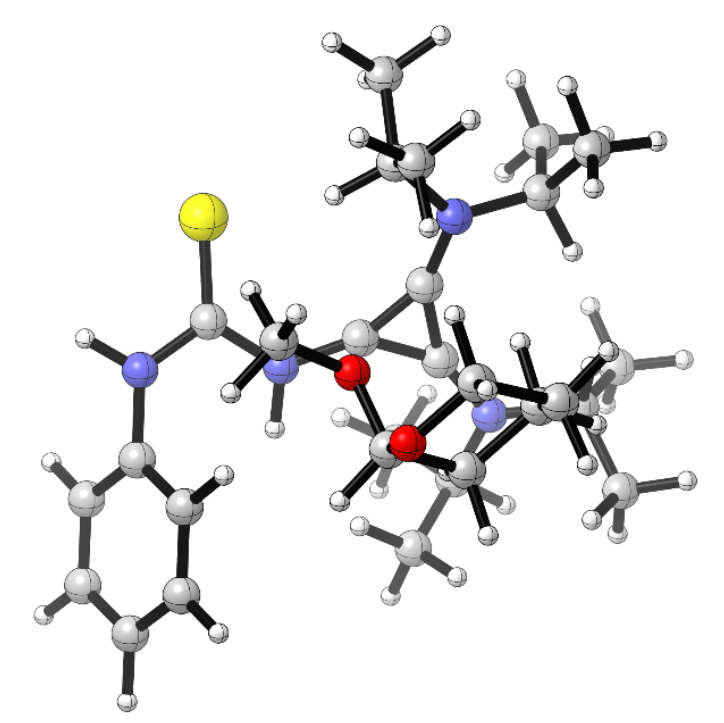

Figure S56. Optimized Structure of E,Z-1-Cy-INT ${ }_{5}$.

- Thermochemistry -

(0 imaginary frequencies)

Zero-point correction $=$

Thermal correction to Energy=

0.731752 (Hartree/Particle)

Thermal correction to Enthalpy=

0.771119

Thermal correction to Gibbs Free Energy=

0.772063

Sum of electronic and zero-point Energies=

0.659882

Sum of electronic and thermal Energies $=$

$-1861.907295$

Sum of electronic and thermal Enthalpies=

$-1861.867928$

$-1861.866983$

Sum of electronic and thermal Free Energies=

$-1861.979165$

$\begin{array}{lrrr}\mathrm{C} & 1.93985000 & -1.31077500 & -1.74895400 \\ \mathrm{~S} & 1.07587100 & -2.26736500 & -2.78921900 \\ \mathrm{~N} & 3.23278300 & -1.54767700 & -1.43533000 \\ \mathrm{H} & 3.65597600 & -2.29488200 & -1.96543400 \\ \mathrm{~N} & 1.43649900 & -0.16728800 & -1.15523700 \\ \mathrm{C} & 4.07356400 & -0.75948400 & -0.59483300 \\ \mathrm{C} & 3.72684900 & -0.52559700 & 0.73567500 \\ \mathrm{C} & 5.25368400 & -0.23672800 & -1.11505000 \\ \mathrm{C} & 4.55354700 & 0.25368100 & 1.53440400 \\ \mathrm{H} & 2.81891800 & -0.96031900 & 1.13652700 \\ \mathrm{C} & 6.08473400 & 0.52259700 & -0.30221200 \\ \mathrm{H} & 5.51224300 & -0.42281700 & -2.15123200 \\ \mathrm{C} & 5.73348500 & 0.77657500 & 1.01840200 \\ \mathrm{H} & 6.38282400 & 1.37366500 & 1.64695600 \\ \mathrm{H} & -0.02172600 & 1.04792700 & 1.80475600 \\ \mathrm{C} & 0.27256700 & -0.86801400 & 2.72396600 \\ \mathrm{C} & -0.41728300 & 0.48810800 & 2.65422600 \\ \mathrm{C} & -1.93462700 & 0.31920300 & 2.57107900 \\ \mathrm{C} & -2.40743400 & -0.56406200 & 3.72681800 \\ \mathrm{C} & -1.63053700 & -1.87296300 & 3.74874600 \\ \mathrm{H} & -2.42989200 & 1.29342900 & 2.61112500 \\ \mathrm{H} & -2.19973500 & -0.14853100 & 1.61624700\end{array}$

$\begin{array}{lrrr}\mathrm{H} & -2.25211100 & -0.03912900 & 4.67499200 \\ \mathrm{H} & -3.47618200 & -0.78080300 & 3.64537400 \\ \mathrm{H} & -1.83902400 & -2.44134100 & 4.65445800 \\ \mathrm{H} & -1.90985200 & -2.49564100 & 2.88987800 \\ \mathrm{O} & -0.22025700 & -1.66712600 & 3.75518200 \\ \mathrm{H} & -0.15586300 & 1.03880300 & 3.56329500 \\ \mathrm{C} & 0.68350400 & -2.77468000 & 1.36610900 \\ \mathrm{H} & 0.69206400 & -3.04511300 & 0.30938700 \\ \mathrm{H} & 0.08614600 & -3.49928000 & 1.92554000 \\ \mathrm{H} & 1.70880500 & -2.80330900 & 1.75586100 \\ \mathrm{O} & 0.13575700 & -1.47002700 & 1.45239400 \\ \mathrm{C} & 0.14632700 & 0.21341000 & -1.05767100 \\ \mathrm{C} & -1.21353900 & 0.01773700 & -0.99919900 \\ \mathrm{C} & -0.65026200 & 1.27712600 & -0.74002600 \\ \mathrm{~N} & -2.28561200 & -0.73671500 & -1.08226700 \\ \mathrm{~N} & -0.73438200 & 2.54442900 & -0.38511500 \\ \mathrm{C} & -3.58717100 & -0.02991300 & -1.19015800 \\ \mathrm{C} & -2.09360400 & -2.20255300 & -1.24957900 \\ \mathrm{C} & -2.01731900 & 3.10744000 & 0.08550400 \\ \mathrm{C} & 0.44670400 & 3.42075100 & -0.55935100 \\ \mathrm{C} & -3.91057200 & 0.33698900 & -2.63874300 \\ \mathrm{H} & -3.43652600 & 0.90319900 & -0.64518400\end{array}$




$\begin{array}{lrrrrrrr}\mathrm{C} & -4.73397000 & -0.75427300 & -0.49969100 & \mathrm{H} & -1.29787700 & 3.38771200 & 2.11565300 \\ \mathrm{C} & -2.66732800 & -2.73094500 & -2.55718000 & \mathrm{H} & -2.13674100 & 4.71384500 & -1.37300600 \\ \mathrm{H} & -2.61628400 & 2.24209200 & 0.37462800 & \mathrm{H} & -2.90599100 & 3.20397000 & -1.89167700 \\ \mathrm{C} & -1.83270100 & 3.94789000 & 1.34570900 & \mathrm{H} & -3.69110300 & 4.23879500 & -0.68576700 \\ \mathrm{C} & -2.72851700 & 3.85859200 & -1.03475500 & \mathrm{H} & 2.32702300 & 3.87222900 & 0.40489300 \\ \mathrm{H} & 0.06384400 & 4.43474100 & -0.43623400 & \mathrm{H} & 1.07067800 & 3.31618700 & 1.52092400 \\ \mathrm{C} & 1.49399300 & 3.17679000 & 0.52498500 & \mathrm{H} & 1.90552400 & 2.16321100 & 0.47686300 \\ \mathrm{C} & 1.01795700 & 3.31738300 & -1.97080900 & \mathrm{H} & 1.47095400 & 2.34133000 & -2.16348800 \\ \mathrm{H} & -3.06230900 & 0.83684300 & -3.11392100 & \mathrm{H} & 0.24112100 & 3.48796400 & -2.71885600 \\ \mathrm{H} & -4.16465300 & -0.54125900 & -3.23279000 & \mathrm{H} & 1.79538700 & 4.07152500 & -2.10716300 \\ \mathrm{H} & -4.76421900 & 1.01883800 & -2.66181100 & \mathrm{H} & 4.28626000 & 0.43161400 & 2.56942400 \\ \mathrm{H} & -5.00331200 & -1.68132700 & -1.00747300 & \mathrm{H} & 7.00611000 & 0.92384400 & -0.70661100 \\ \mathrm{H} & -4.49748300 & -0.97630400 & 0.54220100 & \mathrm{H} & -1.01209700 & -2.30769400 & -1.31993500 \\ \mathrm{H} & -5.61201000 & -0.10534600 & -0.51843700 & \mathrm{C} & -2.54309700 & -2.99823100 & -0.02779400 \\ \mathrm{H} & -3.75946800 & -2.71779800 & -2.57147100 & \mathrm{H} & -3.62443400 & -3.11605000 & 0.03839300 \\ \mathrm{H} & -2.29091200 & -2.16190900 & -3.40916300 & \mathrm{H} & -2.10540200 & -3.99783300 & -0.08530600 \\ \mathrm{H} & -2.35052900 & -3.76872400 & -2.68225000 & \mathrm{H} & -2.17433700 & -2.51989300 & 0.87956400 \\ \mathrm{H} & -1.29666600 & 4.88013100 & 1.15324300 & \mathrm{H} & 1.34030600 & -0.76066500 & 2.95839400 \\ \mathrm{H} & -2.81396800 & 4.21640700 & 1.74185200 & \mathrm{H} & 2.10074400 & 0.43715400 & -0.68840000\end{array}$

Table S3. Energy for all structures at TZVP and 6-311G(d,p) basis sets with thermal corrections and quasi-harmonic approximation adjustments to both TZVP and 6-311G(d,p) energies. All values are in kcal mol-1.

\begin{tabular}{|c|c|c|c|c|c|c|}
\hline Structure & TZVP Energy & $\begin{array}{l}\text { Gibbs Free } \\
\text { Energy } \\
\text { Correction } \\
\text { (TZVP) } \\
\end{array}$ & $\begin{array}{c}\text { 6-311G(d,p) } \\
\text { Energy }\end{array}$ & $\begin{array}{l}\text { Gibbs Free } \\
\text { Energy } \\
\text { Correction } \\
(6-311 \mathrm{G}(\mathrm{d}, \mathrm{p})) \\
\end{array}$ & $\begin{array}{l}\text { Quasi-Harmonic } \\
\text { Approximation } \\
\text { Adjusted Energy } \\
\text { (TZVP) }\end{array}$ & $\begin{array}{l}\text { Quasi-Harmonic } \\
\text { Approximation } \\
\text { Adjusted Energy } \\
(6-311 \mathrm{G}(\mathrm{d}, \mathrm{p})) \\
\end{array}$ \\
\hline $\mathrm{MeOH}$ & -72624.20071 & -72605.98501 & -72612.57677 & -72594.36107 & -72605.99002 & -72597.47924 \\
\hline DHP & -169775.2694 & -169715.5741 & -169760.6547 & -169700.9594 & -169715.6153 & -169701.0002 \\
\hline MTP & -242419.5935 & -242326.2874 & -242399.177 & -242305.8709 & -242326.4628 & -242306.0459 \\
\hline E,E-1 & -926496.2265 & -926189.8496 & -926452.0687 & -926145.6918 & -926189.7402 & -926145.5824 \\
\hline E,Z-1 & -926496.6179 & -926192.8634 & -926451.2628 & -926147.5082 & -926191.8925 & -926146.5375 \\
\hline Z,Z-1 & -926492.7109 & -926189.4395 & -926447.3232 & -926144.0518 & -926188.4074 & -926143.0197 \\
\hline Z,E-1 & -926495.0403 & -926189.9448 & -926450.1953 & -926145.0998 & -926189.4378 & -926144.5928 \\
\hline \multicolumn{7}{|l|}{ Pathway 1} \\
\hline $\begin{array}{c}\text { Z,Z-1- } \\
\text { INT1 }\end{array}$ & -999128.315 & -998792.6602 & -999077.5048 & -998741.8501 & -998792.3091 & -998741.4992 \\
\hline $\begin{array}{l}\text { Z,Z-1- } \\
\text { INT2 }\end{array}$ & -1168911.578 & -1168502.446 & -1168848.181 & -1168439.049 & -1168501.831 & -1168438.434 \\
\hline Z,Z-1-TS & -1168879.085 & -1168471.106 & -1168816.262 & -1168408.283 & -1168470.583 & -1168407.759 \\
\hline $\begin{array}{l}\text { Z,Z-1- } \\
\text { INT3 }\end{array}$ & -1168922.778 & -1168509.364 & -1168860.363 & -1168446.949 & -1168509.115 & -1168446.701 \\
\hline \multicolumn{7}{|l|}{$\begin{array}{c}\text { Pathway } 2 \\
\text { - An }\end{array}$} \\
\hline $\begin{array}{c}\text { E,E-1-An- } \\
\text { INT1 }\end{array}$ & -999127.0463 & -998793.0852 & -999072.9582 & -998738.997 & -998789.3122 & -998738.9051 \\
\hline
\end{tabular}




\begin{tabular}{|c|c|c|c|c|c|c|}
\hline $\begin{array}{l}\text { E,E-1-An- } \\
\text { INT2 }\end{array}$ & -1168905.985 & -1168497.665 & -1168842.373 & -1168434.053 & -1168496.611 & -1168432.999 \\
\hline $\begin{array}{c}\text { E,E-1-An- } \\
\text { TS1 }\end{array}$ & -1168882.623 & -1168475.611 & -1168819.311 & -1168412.3 & -1168475.379 & -1168412.068 \\
\hline $\begin{array}{l}\text { E,E-1-An- } \\
\text { INT3 }\end{array}$ & -1168902.09 & -1168492.009 & -1168838.698 & -1168428.617 & -1168491.658 & -1168428.266 \\
\hline $\begin{array}{c}\text { E,E-1-An- } \\
\text { TS2 }\end{array}$ & -1168883.396 & -1168476.697 & -1168820.346 & -1168413.647 & -1168475.99 & -1168412.94 \\
\hline $\begin{array}{l}\text { E,E-1-An- } \\
\text { INT4 }\end{array}$ & -1168901.839 & -1168492.867 & -1168838.368 & -1168429.396 & -1168492.11 & -1168428.639 \\
\hline $\begin{array}{c}\text { E,E-1-An- } \\
\text { TS3-PA }\end{array}$ & -1168891.163 & -1168480.177 & -1168828.421 & -1168417.435 & -1168479.553 & -1168416.811 \\
\hline $\begin{array}{c}\text { E,E-1-An- } \\
\text { TS3-MA }\end{array}$ & -999107.6866 & -998774.176 & -999056.6096 & -998723.099 & -998773.9547 & -998722.8775 \\
\hline $\begin{array}{l}\text { E,E-1-An- } \\
\text { INT5 }\end{array}$ & -1168920.928 & -1168509.03 & -1168858.189 & -1168446.291 & -1168508.265 & -1168445.526 \\
\hline $\begin{array}{l}\text { E,Z-1-An- } \\
\text { INT1 }\end{array}$ & -999126.0935 & -998791.4973 & -999074.0016 & -998739.4054 & -998790.7014 & -998738.6095 \\
\hline $\begin{array}{l}\text { E,Z-1-An- } \\
\text { INT2 }\end{array}$ & -1168909.942 & -1168500.925 & -1168846.833 & -1168437.815 & -1168500.194 & -1168437.085 \\
\hline $\begin{array}{c}\text { E,Z-1-An- } \\
\text { TS1 }\end{array}$ & -1168882.137 & -1168476.602 & -1168817.581 & -1168412.047 & -1168475.543 & -1168410.987 \\
\hline $\begin{array}{c}\text { E,Z-1-An- } \\
\text { INT3 }\end{array}$ & -1168893.103 & -1168485.444 & -1168828.396 & -1168420.737 & -1168484.238 & -1168419.530 \\
\hline $\begin{array}{c}\text { E,Z-1-An- } \\
\text { TS2 }\end{array}$ & -1168884.827 & -1168476.58 & -1168819.927 & -1168411.68 & -1168476.013 & -1168411.113 \\
\hline $\begin{array}{l}\text { E,Z-1-An- } \\
\text { INT4 }\end{array}$ & -1168899.388 & -1168490.077 & -1168835.194 & -1168425.883 & -1168489.131 & -1168424.937 \\
\hline $\begin{array}{c}\text { E,Z-1-An- } \\
\text { TS3-PA }\end{array}$ & -1168889.999 & -1168480.53 & -1168825.856 & -1168416.387 & -1168479.235 & -1168415.091 \\
\hline $\begin{array}{c}\text { E,Z-1-An- } \\
\text { TS3-MA }\end{array}$ & -999108.1475 & -998776.4114 & -999056.034 & -998724.298 & -998775.7469 & -998723.6336 \\
\hline $\begin{array}{l}\text { E,Z-1-An- } \\
\text { INT5 }\end{array}$ & -1168920.722 & -1168512.326 & -1168856.733 & -1168448.337 & -1168510.177 & -1168446.187 \\
\hline $\begin{array}{l}\text { Z,E-1-An- } \\
\text { INT1 }\end{array}$ & -999124.8228 & -998787.0433 & -999074.3158 & -998736.5363 & -998787.1623 & -998736.6549 \\
\hline $\begin{array}{l}\text { Z,E-1-An- } \\
\text { INT2 }\end{array}$ & -1168912.417 & -1168502.213 & -1168848.924 & -1168438.72 & -1168502.07 & -1168438.577 \\
\hline $\begin{array}{c}\text { Z,E-1-An- } \\
\text { TS1 }\end{array}$ & -1168885.107 & -1168477.615 & -1168822.049 & -1168414.557 & -1168477.443 & -1168414.385 \\
\hline $\begin{array}{l}\text { Z,E-1-An- } \\
\text { INT3 }\end{array}$ & -1168887.948 & -1168479.479 & -1168824.922 & -1168416.453 & -1168479.285 & -1168416.259 \\
\hline $\begin{array}{l}\text { Z,E-1-An- } \\
\text { TS2 } \\
\end{array}$ & -1168887.396 & -1168477.391 & -1168824.352 & -1168414.347 & -1168477.268 & -1168414.224 \\
\hline $\begin{array}{c}\text { Z,E-1-An- } \\
\text { INT4 }\end{array}$ & -1168909.98 & -1168499.273 & -1168846.89 & -1168436.183 & -1168499.149 & -1168436.059 \\
\hline $\begin{array}{c}\text { Z,E-1-An- } \\
\text { TS3-PA }\end{array}$ & -1168888.691 & -1168475.275 & -1168826.279 & -1168412.863 & -1168475.294 & -1168412.883 \\
\hline $\begin{array}{c}\text { Z,E-1-An- } \\
\text { TS3-MA }\end{array}$ & -999093.9807 & -998758.5481 & -999043.2312 & -998707.7986 & -998758.389 & -998707.6393 \\
\hline $\begin{array}{l}\text { Z,E-1-An- } \\
\text { INT5 }\end{array}$ & -1168929.241 & -1168515.001 & -1168867.417 & -1168453.178 & -1168514.777 & -1168452.954 \\
\hline $\begin{array}{l}\text { Z,Z-1-An- } \\
\text { INT1 }\end{array}$ & -999121.7656 & -998788.5957 & -999070.2989 & -998737.1291 & -998787.8281 & -998736.3618 \\
\hline $\begin{array}{c}\text { Z,Z-1-An- } \\
\text { INT2 }\end{array}$ & -1168910.254 & -1168500.861 & -1168846.963 & -1168437.57 & -1168500.257 & -1168436.966 \\
\hline $\begin{array}{c}\text { Z,Z-1-An- } \\
\text { TS1 }\end{array}$ & -1168880.133 & -1168473.374 & -1168816.595 & -1168409.835 & -1168473.078 & -1168409.539 \\
\hline $\begin{array}{c}\text { Z,Z-1-An- } \\
\text { INT3 }\end{array}$ & -1168883.765 & -1168475.622 & -1168820.092 & -1168411.949 & -1168475.248 & -1168411.574 \\
\hline
\end{tabular}




\begin{tabular}{|c|c|c|c|c|c|c|}
\hline $\begin{array}{c}\text { Z,Z-1-An- } \\
\text { TS2 } \\
\end{array}$ & -1168882.218 & -1168473.682 & -1168818.405 & -1168409.87 & -1168473.401 & -1168409.588 \\
\hline $\begin{array}{c}\text { Z,Z-1-An- } \\
\text { INT4 }\end{array}$ & -1168904.284 & -1168495.303 & -1168839.843 & -1168430.863 & -1168494.359 & -1168429.917 \\
\hline $\begin{array}{c}\text { Z,Z-1-An- } \\
\text { TS3-PA }\end{array}$ & -1168881.908 & -1168470.866 & -1168818.274 & -1168407.231 & -1168469.841 & -1168406.207 \\
\hline $\begin{array}{c}\text { Z,Z-1-An- } \\
\text { TS3-MA }\end{array}$ & -999090.3865 & -998757.024 & -999039.1985 & -998705.836 & -998756.2498 & -998705.0621 \\
\hline $\begin{array}{c}\text { Z,Z-1-An- } \\
\text { INT5 }\end{array}$ & -1168928.83 & -1168517.323 & -1168865.999 & -1168454.492 & -1168516.386 & -1168453.555 \\
\hline \multicolumn{7}{|l|}{$\begin{array}{c}\text { Pathway } \\
2-\mathrm{Cy}\end{array}$} \\
\hline $\begin{array}{c}\text { E,Z-1-Cy- } \\
\text { INT1 }\end{array}$ & -999126.0935 & -998791.4973 & -999074.0016 & -998739.4054 & -998790.7014 & -998738.6095 \\
\hline $\begin{array}{c}\text { E,Z-1-Cy- } \\
\text { INT2 }\end{array}$ & -1168907.24 & -1168499.098 & -1168843.775 & -1168435.634 & -1168498.482 & -1168435.017 \\
\hline $\begin{array}{c}\text { E,Z-1-Cy- } \\
\text { TS1 }\end{array}$ & -1168887.459 & -1168479.264 & -1168824.69 & -1168416.495 & -1168479.339 & -1168416.57 \\
\hline $\begin{array}{c}\text { E,Z-1-Cy- } \\
\text { INT3 }\end{array}$ & -1168892.324 & -1168483.705 & -1168829.522 & -1168420.903 & -1168483.385 & -1168420.584 \\
\hline $\begin{array}{c}\text { E,Z-1-Cy- } \\
\text { TS2 }\end{array}$ & -1168891.755 & -1168481.113 & -1168828.299 & -1168417.658 & -1168480.414 & -1168416.959 \\
\hline $\begin{array}{l}\text { E,Z-1-Cy- } \\
\text { INT4 }\end{array}$ & -1168890.695 & -1168496.511 & -1168765.091 & -1168370.907 & -1168496.196 & -1168430.287 \\
\hline $\begin{array}{c}\text { E,Z-1-Cy- } \\
\text { TS3-PA }\end{array}$ & -1168894.233 & -1168481.674 & -1168831.215 & -1168418.656 & -1168481.123 & -1168418.104 \\
\hline $\begin{array}{c}\text { E,Z-1-Cy- } \\
\text { TS3-MA }\end{array}$ & -999109.604 & -998775.4546 & -999058.5815 & -998724.4321 & -998775.1012 & -998724.0785 \\
\hline $\begin{array}{c}\text { E,Z-1-Cy- } \\
\text { INT5 } \\
\end{array}$ & -1168925.145 & -1168511.07 & -1168861.773 & -1168447.697 & -1168510.771 & -1168447.399 \\
\hline
\end{tabular}

\section{Natural Bond Order (NBO) Studies}

Table S4. NBO values for Selected Atoms.

\begin{tabular}{|c|c|c|}
\hline Structure & Atom & NBO Charge (e) \\
\hline \multirow{2}{*}{ Z,Z-1-INT } & $\mathrm{N}_{3}$ & -0.671 \\
\cline { 2 - 3 }$(\mathrm{N}-\mathrm{H} \cdots$ O-H Bond $)$ & $\left(\mathrm{N}_{3}\right)-\mathrm{H}$ & 0.459 \\
\cline { 2 - 3 } b/w catalyst and pyran & $\mathrm{O}$ & -0.795 \\
\cline { 2 - 3 } & $\mathrm{H}$ & 0.510 \\
\hline
\end{tabular}




\section{Pyranylation Product Energies}

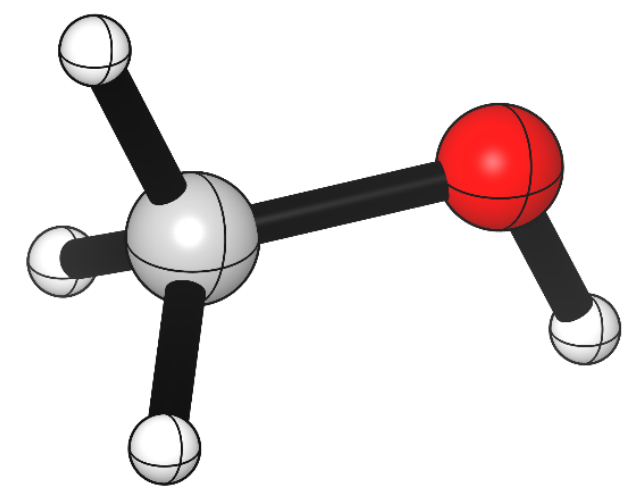

Figure S57. Optimized Structure of $\mathrm{MeOH}$.

- Thermochemistry -

(0 imaginary frequencies)

Zero-point correction $=$

Thermal correction to Energy=

0.051761 (Hartree/Particle)

Thermal correction to Enthalpy=

0.055076

Thermal correction to Gibbs Free Energy=

0.056020

Sum of electronic and zero-point Energies=

0.029029

Sum of electronic and thermal Energies=

$-115.665493$

Sum of electronic and thermal Enthalpies=

$-115.662178$

Sum of electronic and thermal Free Energies=

$-115.661234$

$-115.688225$

$\begin{array}{lrrrrrrr}\mathrm{C} & -0.65848600 & -0.01956200 & 0.00000100 & \mathrm{O} & 0.74535300 & 0.12143700 & 0.00000100 \\ \mathrm{H} & -1.02940500 & -0.54247300 & 0.89176100 & \mathrm{H} & 1.12896600 & -0.75460500 & -0.00000400 \\ \mathrm{H} & -1.02938900 & -0.54257800 & -0.89170500 & \mathrm{H} & -1.08208100 & 0.98552700 & -0.00006800\end{array}$




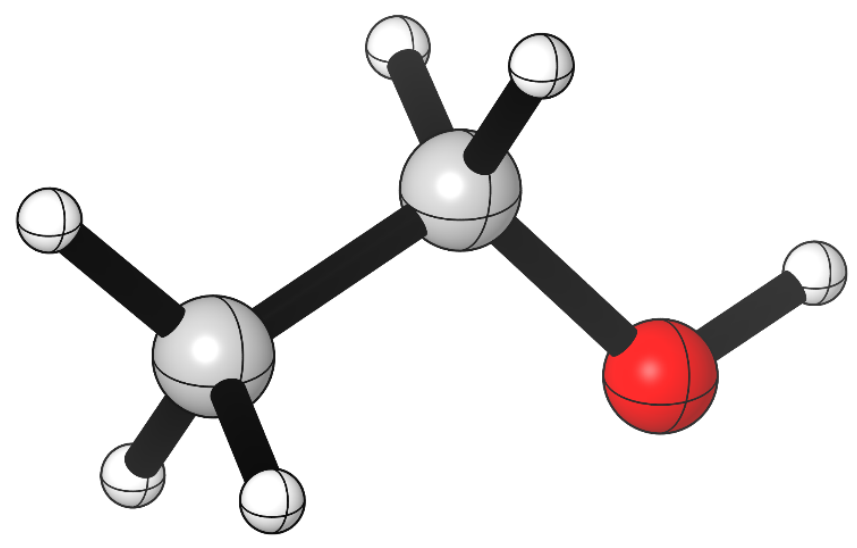

Figure S58. Optimized Structure of EtOH.

- Thermochemistry -

(0 imaginary frequencies)

Zero-point correction=

Thermal correction to Energy=

0.080577 (Hartree/Particle)

0.084854

0.085798

Thermal correction to Enthalpy=

0.055208

Thermal correction to Gibbs Free Energy=

$-154.954306$

Sum of electronic and zero-point Energies=

$-154.950030$

Sum of electronic and thermal Enthalpies=

$-154.949086$

Sum of electronic and thermal Free Energies=

$-154.979675$

$\begin{array}{lrrrrrrr}\mathrm{C} & 0.08772500 & 0.54621800 & 0.00007000 & \mathrm{H} & 1.97341500 & 0.08239500 & -0.00024000 \\ \mathrm{H} & 0.13728900 & 1.19389800 & -0.88720800 & \mathrm{H} & -1.28157300 & -0.85777200 & 0.88519600 \\ \mathrm{H} & 0.13741500 & 1.19366100 & 0.88751300 & \mathrm{H} & -2.06842100 & 0.46476500 & 0.00020100 \\ \mathrm{O} & 1.14526800 & -0.39624300 & -0.00012900 & \mathrm{H} & -1.28168800 & -0.85755400 & -0.88522000 \\ \mathrm{C} & -1.21748900 & -0.22112600 & 0.00006100 & & & \end{array}$

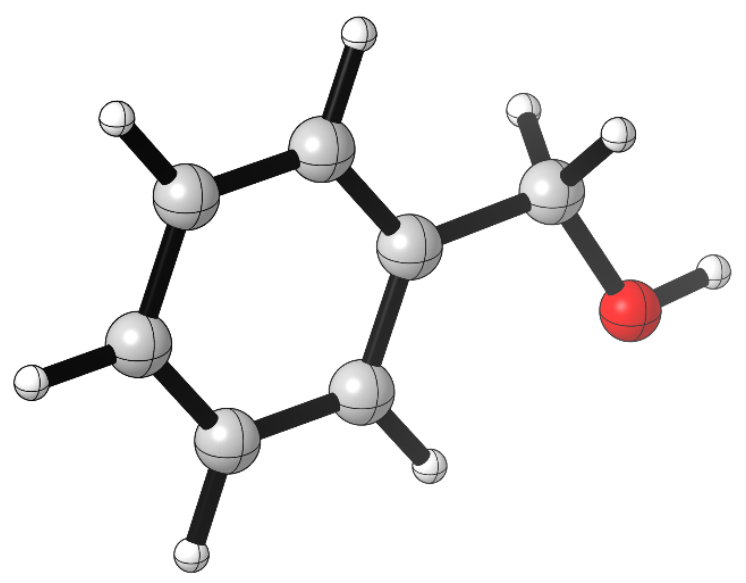


Figure S59. Optimized Structure of $\mathrm{BnOH}$.

- Thermochemistry -

(0 imaginary frequencies)

Zero-point correction=

Thermal correction to Energy=

0.134121 (Hartree/Particle)

Thermal correction to Enthalpy=

0.141295

0.142239

Thermal correction to Gibbs Free Energy=

0.102038

Sum of electronic and zero-point Energies=

$-346.608470$

Sum of electronic and thermal Energies=

$-346.601297$

Sum of electronic and thermal Enthalpies=

$-346.600352$

Sum of electronic and thermal Free Energies=

$-346.640554$

$\begin{array}{lrrrrrrr}\mathrm{C} & -1.89903800 & 0.62532200 & 0.06335800 & \mathrm{H} & -0.74567900 & -1.83939700 & 0.05366300 \\ \mathrm{H} & -2.11364300 & 1.11282500 & 1.02590300 & \mathrm{C} & 1.87217400 & 0.99715300 & -0.02650200 \\ \mathrm{H} & -2.10895800 & 1.35668200 & -0.73036800 & \mathrm{H} & 0.19245800 & 2.33037100 & -0.02644200 \\ \mathrm{O} & -2.68077700 & -0.53828500 & -0.09748400 & \mathrm{C} & 2.29493100 & -0.32810700 & -0.01380400 \\ \mathrm{C} & -0.43272300 & 0.27329500 & 0.02279500 & \mathrm{H} & 1.67429400 & -2.38259100 & 0.02203000 \\ \mathrm{C} & -0.00606000 & -1.04957500 & 0.03425800 & \mathrm{H} & 2.60057800 & 1.79995700 & -0.05248500 \\ \mathrm{C} & 0.51697800 & 1.29348300 & -0.01073000 & \mathrm{H} & 3.35353300 & -0.56162600 & -0.02928500 \\ \mathrm{C} & 1.35265000 & -1.34688600 & 0.01500600 & \mathrm{H} & -3.59984100 & -0.29805900 & 0.01057300\end{array}$

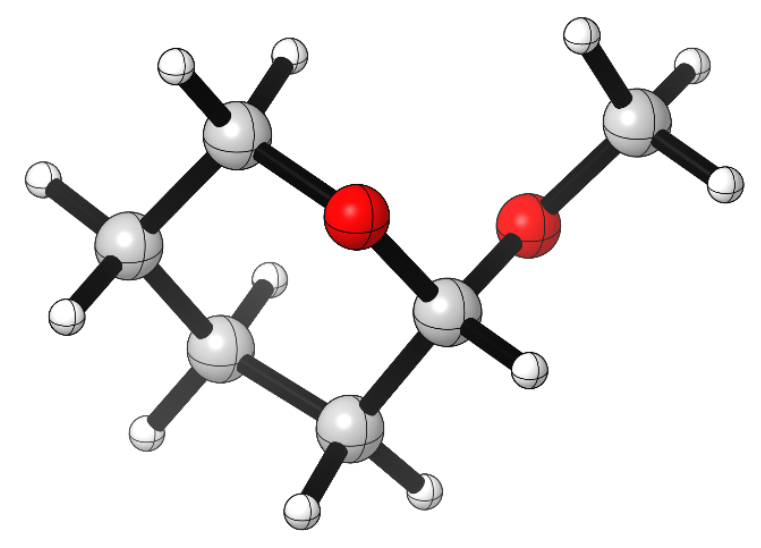

Figure S60. Optimized Structure of DHP-MeOH (Axial).

- Thermochemistry -

(0 imaginary frequencies)

Zero-point correction=

0.180759 (Hartree/Particle) 
Thermal correction to Energy=

Thermal correction to Enthalpy=

Thermal correction to Gibbs Free Energy=

Sum of electronic and zero-point Energies=

Sum of electronic and thermal Energies=

Sum of electronic and thermal Enthalpies=

Sum of electronic and thermal Free Energies=
0.188582

0.189526

0.148695

$-386.104437$

$-386.096615$

$-386.095670$

$-386.136501$

$\begin{array}{rrrrrrrr}\mathrm{H} & -1.12543900 & -1.45597400 & 1.44154200 & \mathrm{H} & -0.17049200 & 1.48610700 & -1.05994100 \\ \mathrm{C} & 0.56123200 & -0.42583800 & 0.65141900 & \mathrm{H} & -0.95875900 & 2.43336700 & 0.21654500 \\ \mathrm{C} & -0.61112100 & -1.38264500 & 0.47788300 & \mathrm{O} & 0.13807400 & 0.89364700 & 0.90044400 \\ \mathrm{C} & -1.57807300 & -0.87401100 & -0.59187500 & \mathrm{H} & 1.15984700 & -0.68535700 & 1.53453400 \\ \mathrm{C} & -1.96737700 & 0.57307400 & -0.28584000 & \mathrm{H} & -0.22107200 & -2.37168700 & 0.22820200 \\ \mathrm{C} & -0.71565000 & 1.42114000 & -0.10893100 & \mathrm{C} & 2.55432100 & 0.21829500 & -0.41977700 \\ \mathrm{H} & -1.08956000 & -0.92351500 & -1.56998500 & \mathrm{H} & 3.17600500 & -0.13813300 & 0.41320900 \\ \mathrm{H} & -2.46352900 & -1.51358700 & -0.63682200 & \mathrm{H} & 2.36634800 & 1.28794000 & -0.28167800 \\ \mathrm{H} & -2.58287100 & 0.99284100 & -1.08724700 & \mathrm{H} & 3.08788800 & 0.05930000 & -1.35638400 \\ \mathrm{H} & -2.55599600 & 0.61161200 & 0.63782900 & \mathrm{O} & 1.35163200 & -0.51402200 & -0.50257900\end{array}$

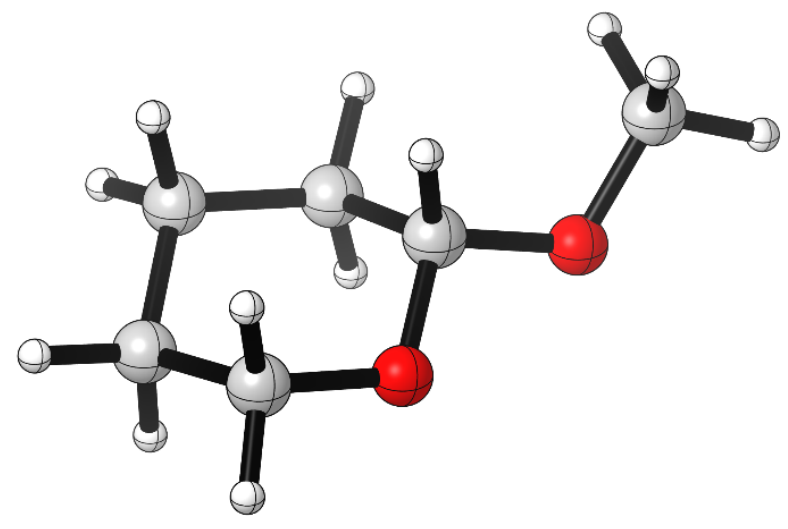

Figure S61. Optimized Structure of DHP-MeOH (Equatorial).

- Thermochemistry -

(0 imaginary frequencies)

Zero-point correction=

Thermal correction to Energy=

Thermal correction to Enthalpy=

Thermal correction to Gibbs Free Energy=

Sum of electronic and zero-point Energies $=$

Sum of electronic and thermal Energies=

Sum of electronic and thermal Enthalpies=

Sum of electronic and thermal Free Energies=
0.179964 (Hartree/Particle)

0.187903

0.188847

0.147471

$-386.095617$

$-386.087678$

$-386.086734$

$-386.128110$

$\mathrm{H} \quad-0.04673600 \quad 1.18393000 \quad-1.40356400$

$\begin{array}{llll}\text { C } & -0.04739700 & 1.18185800 & -0.30913200\end{array}$

C $\quad 0.57294900 \quad-0.14111300 \quad 0.13213100$

$\begin{array}{llll}\mathrm{C} & -1.47309100 & 1.30968600 & 0.23092500\end{array}$ 


$\begin{array}{lrrrrrrr}\mathrm{C} & -2.27829600 & 0.06289900 & -0.13728200 & \mathrm{O} & -0.21534000 & -1.22428900 & -0.27658800 \\ \mathrm{C} & -1.51466600 & -1.18850200 & 0.28169100 & \mathrm{H} & 0.66863200 & -0.16066100 & 1.23787800 \\ \mathrm{H} & -1.44204500 & 1.41297400 & 1.32320000 & \mathrm{H} & 0.57716700 & 2.01391300 & 0.02977600 \\ \mathrm{H} & -1.95083500 & 2.21253700 & -0.15750100 & \mathrm{C} & 2.89502600 & 0.16052100 & 0.29011300 \\ \mathrm{H} & -3.26149100 & 0.07210400 & 0.34262000 & \mathrm{H} & 2.95226800 & -0.30008300 & 1.28636600 \\ \mathrm{H} & -2.43724100 & 0.03148900 & -1.22031400 & \mathrm{H} & 2.83696900 & 1.25099500 & 0.41031600 \\ \mathrm{H} & -1.44711900 & -1.23382400 & 1.38170600 & \mathrm{H} & 3.80417100 & -0.07843600 & -0.26075700 \\ \mathrm{H} & -2.01233400 & -2.09571900 & -0.06231600 & \mathrm{O} & 1.81927100 & -0.35337500 & -0.45317300\end{array}$

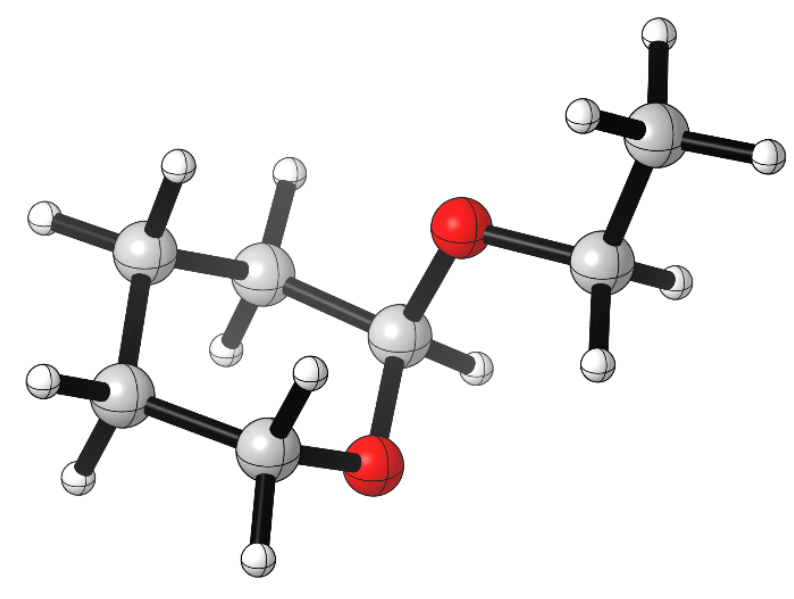

Figure S62. Optimized Structure of DHP-EtOH (Axial).

- Thermochemistry -

(0 imaginary frequencies)

Zero-point correction=

Thermal correction to Energy=

0.209412 (Hartree/Particle)

Thermal correction to Enthalpy=

0.218468

Thermal correction to Gibbs Free Energy=

0.219412

Sum of electronic and zero-point Energies=

0.175333

Sum of electronic and thermal Energies=

$-425.393203$

Sum of electronic and thermal Enthalpies=

$-425.384147$

Sum of electronic and thermal Free Energies=

$-425.383203$

$-425.427282$

$\begin{array}{rrrrrrrr}\mathrm{H} & 1.85685900 & -1.45915900 & -1.23979700 & \mathrm{O} & 0.50067800 & 0.88506800 & -1.03750100 \\ \mathrm{C} & 0.02622900 & -0.43189100 & -0.88092400 & \mathrm{H} & -0.35156300 & -0.70007100 & -1.87587100 \\ \mathrm{C} & 1.12514000 & -1.38474000 & -0.42898200 & \mathrm{H} & 0.68919000 & -2.37410700 & -0.27437400 \\ \mathrm{C} & 1.80378800 & -0.86646000 & 0.83972900 & \mathrm{C} & -2.17880900 & 0.20092500 & -0.30870600 \\ \mathrm{C} & 2.25826200 & 0.57791200 & 0.62324700 & \mathrm{H} & -2.56743200 & -0.20155600 & -1.25616400 \\ \mathrm{C} & 1.08721200 & 1.42397900 & 0.14175200 & \mathrm{H} & -1.94222200 & 1.25848200 & -0.47183900 \\ \mathrm{H} & 1.09022300 & -0.90555500 & 1.66852400 & \mathrm{O} & -1.00903800 & -0.50831900 & 0.05960500 \\ \mathrm{H} & 2.65002000 & -1.50510500 & 1.10633300 & \mathrm{C} & -3.19674000 & 0.03706500 & 0.79888200 \\ \mathrm{H} & 2.66419700 & 1.00594900 & 1.54488500 & \mathrm{H} & -4.12528100 & 0.55355200 & 0.54375800 \\ \mathrm{H} & 3.05172900 & 0.60767500 & -0.13206000 & \mathrm{H} & -3.41776300 & -1.02006700 & 0.96077200 \\ \mathrm{H} & 0.32812300 & 1.49781000 & 0.93182500 & \mathrm{H} & -2.81344500 & 0.45445200 & 1.73277300 \\ \mathrm{H} & 1.40375300 & 2.43296300 & -0.12559600 & & & & \end{array}$




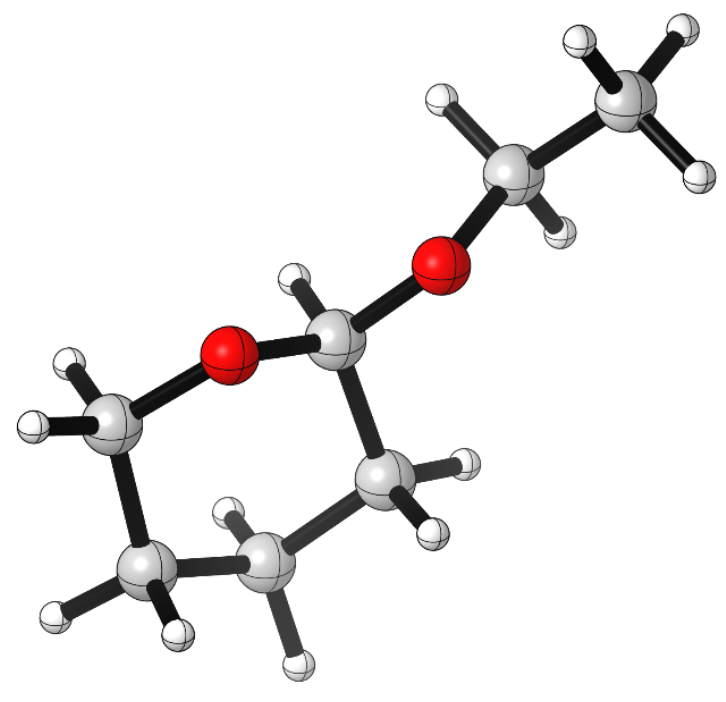

Figure S63. Optimized Structure of DHP-EtOH (Equatorial).

- Thermochemistry -

(0 imaginary frequencies)

Zero-point correction $=$ Thermal correction to Energy= 0.208533 (Hartree/Particle)

Thermal correction to Enthalpy=

0.217773

0.218717

Thermal correction to Gibbs Free Energy=

0.174003

Sum of electronic and zero-point Energies=

$-425.384606$

Sum of electronic and thermal Energies=

$-425.375367$

Sum of electronic and thermal Enthalpies=

$-425.374423$

Sum of electronic and thermal Free Energies=

$-425.419137$

$\begin{array}{rrrr}\mathrm{H} & -0.50183900 & 1.20079900 & -1.37679800 \\ \mathrm{C} & 0.08317700 & -0.10270300 & 0.19021800 \\ \mathrm{C} & -0.55623900 & 1.19879800 & -0.28374300 \\ \mathrm{C} & -2.00971500 & 1.28280500 & 0.18662400 \\ \mathrm{C} & -2.75776600 & 0.01026500 & -0.21240500 \\ \mathrm{C} & -1.97527600 & -1.21646200 & 0.24421600 \\ \mathrm{H} & -2.03458900 & 1.39084000 & 1.27865600 \\ \mathrm{H} & -2.49669800 & 2.16892500 & -0.22806200 \\ \mathrm{H} & -3.76077400 & -0.01060900 & 0.22432200 \\ \mathrm{H} & -2.86868600 & -0.02843100 & -1.30117300 \\ \mathrm{H} & -1.95893100 & -1.25889800 & 1.34636500 \\ \mathrm{H} & -2.42865900 & -2.13852700 & -0.12103700\end{array}$

$\mathrm{O}$

$\mathrm{H}$

$\mathrm{H}$

C

$\mathrm{H}$

$\mathrm{H}$

$\mathrm{O}$

C

$\mathrm{H}$

$\mathrm{H}$

$\mathrm{H}$

$\begin{array}{rrc}-0.65091000 & -1.21215600 & -0.25210300 \\ 0.12449300 & -0.11833200 & 1.29872900 \\ 0.02678500 & 2.04862100 & 0.08390800 \\ 2.40114100 & 0.20473500 & 0.49527900 \\ 2.37479500 & -0.31576500 & 1.46414700 \\ 2.27753200 & 1.27980300 & 0.69306500 \\ 1.36204700 & -0.27103100 & -0.33397600 \\ 3.71749700 & -0.04721200 & -0.20702800 \\ 4.55030600 & 0.30139900 & 0.40864600 \\ 3.84518900 & -1.11450300 & -0.39779000 \\ 3.74507600 & 0.47882600 & -1.16332100\end{array}$




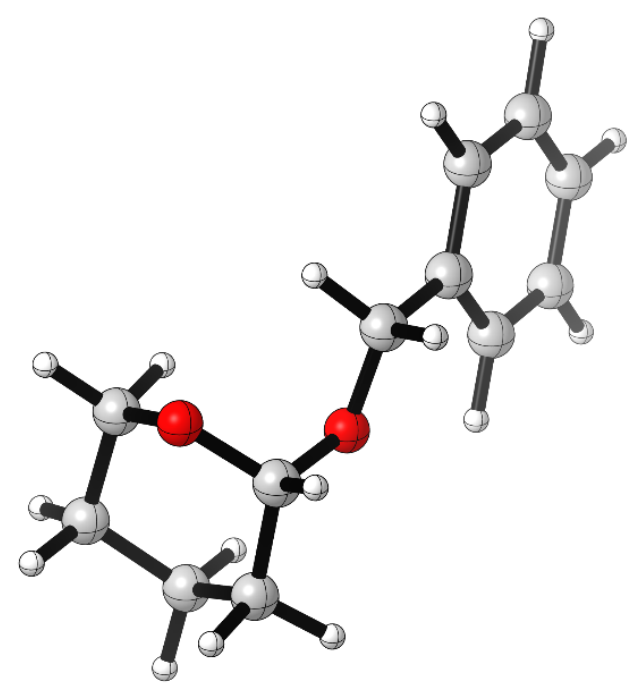

Figure S64. Optimized Structure of DHP-BnOH (Axial).

- Thermochemistry -

(0 imaginary frequencies)

Zero-point correction=

Thermal correction to Energy=

0.262742 (Hartree/Particle)

Thermal correction to Enthalpy=

0.274008

0.274953

Thermal correction to Gibbs Free Energy=

0.224261

Sum of electronic and zero-point Energies=

$-617.048075$

Sum of electronic and thermal Energies=

$-617.036808$

Sum of electronic and thermal Enthalpies=

$-617.035864$

Sum of electronic and thermal Free Energies=

$-617.086556$

$\begin{array}{crrr}\mathrm{H} & -3.72894600 & 0.50925200 & -1.45102600 \\ \mathrm{C} & -1.84416700 & -0.37185800 & -1.01207100 \\ \mathrm{C} & -2.76718300 & 0.83837600 & -1.04468300 \\ \mathrm{C} & -2.96286600 & 1.41287500 & 0.35893700 \\ \mathrm{C} & -3.39551500 & 0.29840100 & 1.31314100 \\ \mathrm{C} & -2.41605400 & -0.86435400 & 1.23448800 \\ \mathrm{H} & -2.02059300 & 1.84721500 & 0.70690800 \\ \mathrm{H} & -3.70460200 & 2.21541500 & 0.33879100 \\ \mathrm{H} & -3.44774100 & 0.66217800 & 2.34369400 \\ \mathrm{H} & -4.39445700 & -0.05874900 & 1.03831000 \\ \mathrm{H} & -1.43440200 & -0.55561800 & 1.61713300 \\ \mathrm{H} & -2.75968700 & -1.71923800 & 1.81778700 \\ \mathrm{O} & -2.28121500 & -1.34789400 & -0.09931800 \\ \mathrm{H} & -1.82992100 & -0.89133300 & -1.97841500 \\ \mathrm{H} & -2.35053800 & 1.58119900 & -1.72854300\end{array}$

$\begin{array}{lrrr}\mathrm{C} & 0.44041000 & -0.90416000 & -0.76281700 \\ \mathrm{H} & 0.53210800 & -1.26645400 & -1.79901400 \\ \mathrm{H} & 0.15964400 & -1.76241000 & -0.14167700 \\ \mathrm{O} & -0.55677700 & 0.09186800 & -0.69958700 \\ \mathrm{C} & 1.75986200 & -0.34396500 & -0.30045000 \\ \mathrm{C} & 1.99170400 & 1.02728900 & -0.25899500 \\ \mathrm{C} & 2.78186900 & -1.21571700 & 0.07106300 \\ \mathrm{C} & 3.22780300 & 1.51739500 & 0.14672000 \\ \mathrm{H} & 1.19580100 & 1.70557700 & -0.53924800 \\ \mathrm{C} & 4.01834400 & -0.72698200 & 0.46991500 \\ \mathrm{H} & 2.60677300 & -2.28747800 & 0.05120300 \\ \mathrm{C} & 4.24481600 & 0.64413300 & 0.50991000 \\ \mathrm{H} & 3.39557200 & 2.58832200 & 0.17976900 \\ \mathrm{H} & 4.80328000 & -1.41740500 & 0.75784500 \\ \mathrm{H} & 5.20750400 & 1.02913600 & 0.82678700\end{array}$




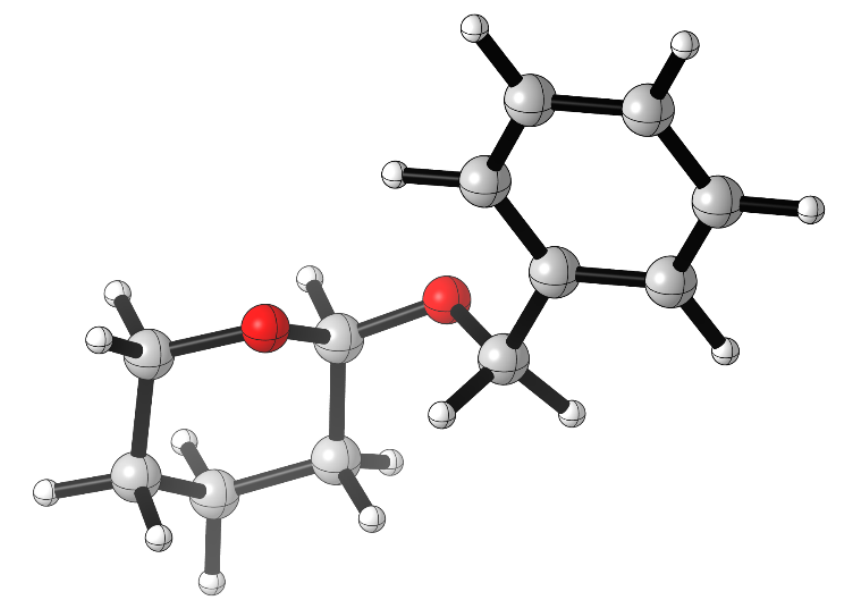

Figure S65. Optimized Structure of DHP-BnOH (Equatorial).

- Thermochemistry -

(0 imaginary frequencies)

Zero-point correction=

Thermal correction to Energy=

0.262530 (Hartree/Particle)

Thermal correction to Enthalpy=

0.274632

0.275577

Thermal correction to Gibbs Free Energy $=\quad 0.222728$

Sum of electronic and zero-point Energies=

Sum of electronic and thermal Energies=

$-617.042306$

Sum of electronic and thermal Enthalpies=

$-617.030204$

Sum of electronic and thermal Free Energies=

$-617.029260$

$-617.082108$

$\begin{array}{crrrrrrr}\mathrm{H} & 2.13159900 & -1.58242000 & -0.83612400 & \mathrm{C} & -0.43994600 & -1.04982900 & -0.21182400 \\ \mathrm{C} & 1.58905600 & -0.26063000 & 0.77091600 & \mathrm{H} & -0.50868900 & -2.13887200 & -0.31214200 \\ \mathrm{C} & 2.53934400 & -1.27232600 & 0.13179700 & \mathrm{H} & 0.06714200 & -0.65133900 & -1.09556200 \\ \mathrm{C} & 3.92229600 & -0.64809100 & -0.06747000 & \mathrm{O} & 0.29903200 & -0.72839900 & 0.96097100 \\ \mathrm{C} & 3.79407600 & 0.66919800 & -0.83248300 & \mathrm{C} & -1.81758400 & -0.44516100 & -0.12915900 \\ \mathrm{C} & 2.77405300 & 1.56745300 & -0.14408500 & \mathrm{C} & -2.95005700 & -1.20147000 & -0.40925400 \\ \mathrm{H} & 4.37777300 & -0.45455500 & 0.91189300 & \mathrm{C} & -1.96224300 & 0.90260600 & 0.19785100 \\ \mathrm{H} & 4.58406500 & -1.34200100 & -0.59144500 & \mathrm{C} & -4.21401300 & -0.62107800 & -0.37542500 \\ \mathrm{H} & 4.75714200 & 1.18465700 & -0.89277300 & \mathrm{H} & -2.84579000 & -2.25515100 & -0.65011800 \\ \mathrm{H} & 3.45947100 & 0.47335100 & -1.85694700 & \mathrm{C} & -3.22171900 & 1.48160100 & 0.23572500 \\ \mathrm{H} & 3.14983700 & 1.86388500 & 0.84876300 & \mathrm{H} & -1.07406600 & 1.48272300 & 0.42245800 \\ \mathrm{H} & 2.58387900 & 2.47574000 & -0.71693100 & \mathrm{C} & -4.35192900 & 0.72172700 & -0.05327300 \\ \mathrm{O} & 1.52174900 & 0.92323300 & -0.00303500 & \mathrm{H} & -5.09044200 & -1.22111200 & -0.59394700 \\ \mathrm{H} & 1.94759500 & -0.00799700 & 1.78002400 & \mathrm{H} & -3.32608100 & 2.53090300 & 0.48912300 \\ \mathrm{H} & 2.58802100 & -2.15696700 & 0.77212800 & \mathrm{H} & -5.33571700 & 1.17647500 & -0.02179000\end{array}$




\section{Higher Energy Conformers}

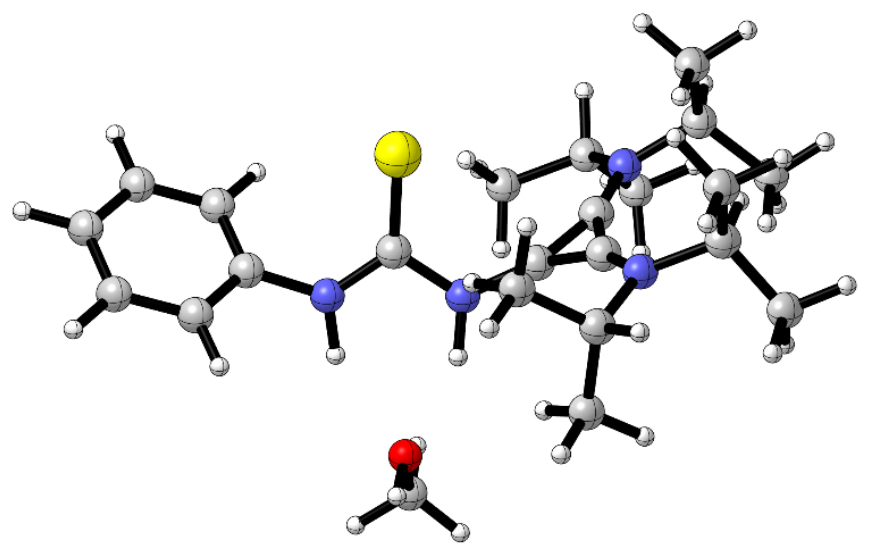

Figure S66. Optimized Higher Energy Structure of $Z, Z-1-I_{N} \mathbf{I N}_{1}$ (1).

- Thermochemistry -

(0 imaginary frequencies)

Zero-point correction $=$

Thermal correction to Energy=

Thermal correction to Enthalpy=

Thermal correction to Gibbs Free Energy=

Sum of electronic and zero-point Energies=

Sum of electronic and thermal Energies $=$

Sum of electronic and thermal Enthalpies=

Sum of electronic and thermal Free Energies=
0.601833 (Hartree/Particle)

0.636808

0.637752

0.532055

$-1591.460281$

$-1591.425306$

$-1591.424362$

$-1591.530059$

C $\quad \begin{array}{llll}1.69297600 & 0.18626800 & -0.13401300\end{array}$

S $\quad \begin{array}{llll}1.36577300 & 0.71678100 & -1.67655500\end{array}$

$\mathrm{N} \quad 2.90457700 \quad-0.06954500 \quad 0.39397400$

$\begin{array}{llll}\mathrm{H} & 2.91873600 & -0.50761800 & 1.30963800\end{array}$

$\begin{array}{llll}\mathrm{N} & 0.67253100 & -0.07593300 & 0.77761300\end{array}$

$\mathrm{H} \quad 0.89183200 \quad-0.66348400 \quad 1.58195000$

$\begin{array}{llll}\mathrm{O} & 2.00776900 & -1.43223600 & 2.90335400\end{array}$

$\mathrm{H} \quad 2.18545000 \quad-2.36543300 \quad 3.03029700$

$\begin{array}{llll}\mathrm{C} & 2.11812200 & -0.75070100 & 4.15638100\end{array}$

$\mathrm{H} \quad 3.11740700 \quad-0.86829200 \quad 4.58162900$

$\begin{array}{llll}\mathrm{H} & 1.94340000 & 0.30516500 & 3.95297000\end{array}$

$\mathrm{H} \quad \begin{array}{llll}1.36878500 & -1.10754000 & 4.86725000\end{array}$

$\begin{array}{lllll}\mathrm{C} & 4.16390400 & 0.04015500 & -0.26305800\end{array}$

$\begin{array}{lllll}\text { C } & 4.56755100 & 1.23235700 & -0.85237600\end{array}$

$\begin{array}{llll}\text { C } & 5.01285300 & -1.06088400 & -0.24678400\end{array}$

$\begin{array}{lllll}\mathrm{C} & 5.82095600 & 1.31097400 & -1.44165200\end{array}$

$\mathrm{H} \quad 3.90164900 \quad 2.08492200 \quad-0.85453400$

$\begin{array}{llll}\mathrm{C} & 6.27166700 & -0.96958800 & -0.82563800\end{array}$

$\mathrm{H} \quad 4.68329800 \quad-1.98818100 \quad 0.20969600$

$\begin{array}{llll}\text { C } & 6.67534200 & 0.21446900 & -1.42876900\end{array}$

$\begin{array}{lrrr}\mathrm{H} & 7.65409400 & 0.28410600 & -1.88788100 \\ \mathrm{H} & 6.13376200 & 2.23738900 & -1.90814400 \\ \mathrm{H} & 6.93294600 & -1.82778800 & -0.81273200 \\ \mathrm{C} & -0.64302500 & 0.05511500 & 0.41129000 \\ \mathrm{C} & -1.71594000 & 0.86530700 & 0.14061300 \\ \mathrm{C} & -1.79350700 & -0.55877300 & 0.00755400 \\ \mathrm{~N} & -2.26633000 & 2.04668600 & 0.02558600 \\ \mathrm{~N} & -2.39782300 & -1.68424100 & -0.28576600 \\ \mathrm{C} & -3.62549700 & 2.20610700 & -0.54579200 \\ \mathrm{C} & -1.59443700 & 3.29110000 & 0.49458000 \\ \mathrm{C} & -3.81206500 & -1.75300500 & -0.70848000 \\ \mathrm{C} & -1.61859400 & -2.94502900 & -0.17767000 \\ \mathrm{H} & -3.77559000 & 3.28666200 & -0.58828500 \\ \mathrm{C} & -4.69747200 & 1.63700300 & 0.38219000 \\ \mathrm{C} & -3.66698400 & 1.70107100 & -1.98562800 \\ \mathrm{C} & -0.11136600 & 3.10710300 & 0.75892200 \\ \mathrm{H} & -1.70517900 & 3.99926500 & -0.33283400 \\ \mathrm{C} & -2.31611500 & 3.83852300 & 1.72462900 \\ \mathrm{H} & -4.15389700 & -0.72193200 & -0.74530600 \\ \mathrm{C} & -3.93842400 & -2.32957700 & -2.11551200\end{array}$




$\begin{array}{lrrrrrrr}\mathrm{C} & -4.65753800 & -2.49341500 & 0.32282800 & \mathrm{H} & -2.25842500 & 3.12211100 & 2.54891500 \\ \mathrm{H} & -2.31212900 & -3.72823800 & -0.48504200 & \mathrm{H} & -1.83963300 & 4.76727800 & 2.04394000 \\ \mathrm{C} & -0.43528000 & -2.95548900 & -1.13866000 & \mathrm{H} & -3.65430400 & -3.38373100 & -2.15776800 \\ \mathrm{C} & -1.21864900 & -3.22137800 & 1.26901500 & \mathrm{H} & -4.97698300 & -2.25826700 & -2.44493400 \\ \mathrm{H} & -4.52630300 & 0.58598100 & 0.62007600 & \mathrm{H} & -3.31561700 & -1.77591400 & -2.82160800 \\ \mathrm{H} & -4.71920400 & 2.18389100 & 1.32620800 & \mathrm{H} & -4.37230000 & -3.54537900 & 0.40515500 \\ \mathrm{H} & -5.68106800 & 1.72233600 & -0.08411300 & \mathrm{H} & -4.56346900 & -2.03214200 & 1.30888400 \\ \mathrm{H} & -2.94490200 & 2.24635600 & -2.59636600 & \mathrm{H} & -5.70865100 & -2.46092700 & 0.02897600 \\ \mathrm{H} & -3.43020700 & 0.63888300 & -2.06851500 & \mathrm{H} & 0.04669000 & -3.93512200 & -1.11415800 \\ \mathrm{H} & -4.66146500 & 1.85727000 & -2.40826800 & \mathrm{H} & -0.75900400 & -2.75850500 & -2.16196400 \\ \mathrm{H} & 0.07030500 & 2.45325000 & 1.61582600 & \mathrm{H} & 0.31267600 & -2.20575000 & -0.87220600 \\ \mathrm{H} & 0.40474800 & 2.70425100 & -0.11295400 & \mathrm{H} & -0.49355100 & -2.48892000 & 1.63419500 \\ \mathrm{H} & 0.31903200 & 4.08282500 & 0.98962900 & \mathrm{H} & -2.08848400 & -3.20657700 & 1.92884200 \\ \mathrm{H} & -3.36726900 & 4.05901900 & 1.52952200 & \mathrm{H} & -0.75322700 & -4.20687500 & 1.33840200\end{array}$

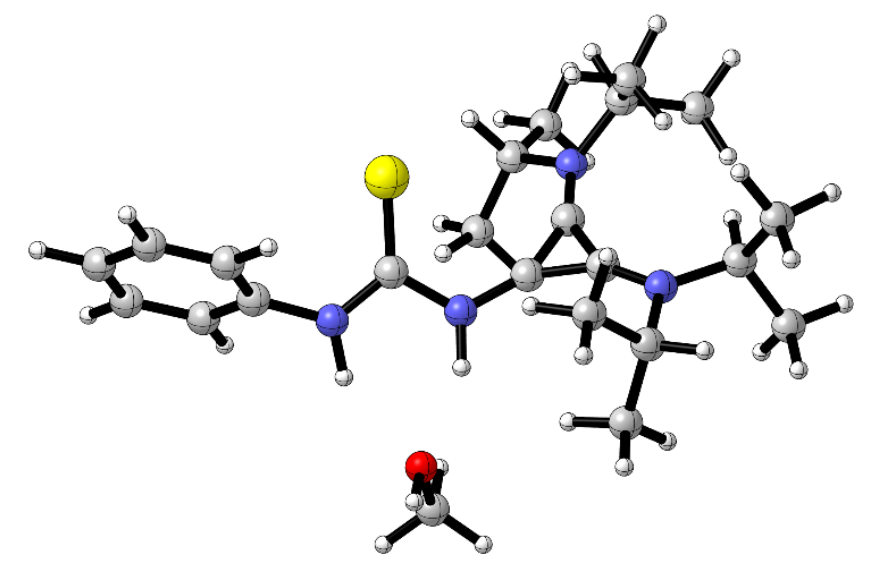

Figure S67. Optimized Higher Energy Structure of Z,Z-1-INT 1 (2).

- Thermochemistry -

(0 imaginary frequencies)

Zero-point correction $=$

Thermal correction to Energy=

0.601828 (Hartree/Particle)

Thermal correction to Enthalpy=

0.636818

0.637763

Thermal correction to Gibbs Free Energy $=0.531900$

Sum of electronic and zero-point Energies $=\quad-1591.460746$

Sum of electronic and thermal Energies $=\quad-1591.425756$

Sum of electronic and thermal Enthalpies $=\quad-1591.424812$

Sum of electronic and thermal Free Energies $=\quad-1591.530675$

$\begin{array}{lccccccc}\mathrm{C} & 1.75691800 & 0.01039700 & -0.16593300 & \mathrm{~N} & 0.66321800 & -0.66017600 & 0.40076900 \\ \mathrm{~S} & 1.56789900 & 1.32662300 & -1.15609800 & \mathrm{H} & 0.81855300 & -1.60091300 & 0.76225300 \\ \mathrm{~N} & 2.91652900 & -0.56696300 & 0.18851400 & \mathrm{O} & 1.79494700 & -2.89730800 & 1.71544900 \\ \mathrm{H} & 2.87155100 & -1.34851000 & 0.83444200 & \mathrm{H} & 2.03466000 & -3.78447300 & 1.44277400\end{array}$




\begin{tabular}{|c|c|c|c|c|c|c|c|}
\hline $\mathrm{C}$ & 1.68185700 & -2.84509800 & 3.14006500 & $\mathrm{H}$ & -4.30714500 & -0.03923600 & -0.15958600 \\
\hline $\mathrm{H}$ & 2.63216100 & -3.09063000 & 3.61947900 & $\mathrm{C}$ & -4.88585900 & -1.36417400 & -1.72898300 \\
\hline $\mathrm{H}$ & 1.41327300 & -1.81934900 & 3.39155500 & $\mathrm{C}$ & -4.94939500 & -1.85434200 & 0.76023500 \\
\hline $\mathrm{H}$ & 0.90025100 & -3.51897500 & 3.49958100 & $\mathrm{H}$ & -3.23263800 & -3.32421100 & -0.97891800 \\
\hline $\mathrm{C}$ & 4.21442500 & -0.17242400 & -0.26235800 & $\mathrm{C}$ & -1.35934100 & -2.79221500 & -1.84775600 \\
\hline $\mathrm{C}$ & 5.13235800 & 0.29132900 & 0.67033900 & $\mathrm{C}$ & -1.77783900 & -3.47025800 & 0.56986700 \\
\hline $\mathrm{C}$ & 4.56367000 & -0.29936300 & -1.60093900 & $\mathrm{H}$ & -0.65079400 & 1.75311400 & 2.79104100 \\
\hline $\mathrm{C}$ & 6.41575700 & 0.62947300 & 0.25959900 & $\mathrm{H}$ & 0.70907400 & 1.57864300 & 1.65162800 \\
\hline $\mathrm{H}$ & 4.83844600 & 0.39606700 & 1.70877400 & $\mathrm{H}$ & 0.58560200 & 3.00709200 & 2.65462600 \\
\hline $\mathrm{C}$ & 5.84305300 & 0.05146900 & -2.00608100 & $\mathrm{H}$ & -2.16559700 & 4.77447600 & 1.05451700 \\
\hline $\mathrm{H}$ & 3.83371700 & -0.65788700 & -2.31599800 & $\mathrm{H}$ & -2.23408600 & 3.77244200 & 2.51739500 \\
\hline $\mathrm{C}$ & 6.77015400 & 0.51279800 & -1.07811100 & $\mathrm{H}$ & -0.84610400 & 4.82223000 & 2.20630300 \\
\hline $\mathrm{H}$ & 7.76954200 & 0.78163600 & -1.39892000 & $\mathrm{H}$ & -4.55454600 & 1.78825600 & 0.43296300 \\
\hline $\mathrm{H}$ & 7.13478300 & 0.99118800 & 0.98474400 & $\mathrm{H}$ & -4.12820600 & 3.35993500 & 1.10357500 \\
\hline $\mathrm{H}$ & 6.11755300 & -0.04010400 & -3.05008600 & $\mathrm{H}$ & -4.91324000 & 3.25784600 & -0.47751600 \\
\hline $\mathrm{C}$ & -0.60478900 & -0.21424500 & 0.26227300 & $\mathrm{H}$ & -1.89625300 & 1.97556100 & -2.33406300 \\
\hline $\mathrm{C}$ & -1.50163400 & 0.82416300 & 0.23770000 & $\mathrm{H}$ & -3.32270200 & 1.04314900 & -1.84199400 \\
\hline $\mathrm{C}$ & -1.91076800 & -0.50145200 & -0.03837800 & $\mathrm{H}$ & -3.52321000 & 2.64053500 & -2.54587300 \\
\hline $\mathrm{N}$ & -1.77574200 & 2.10788500 & 0.34673400 & $\mathrm{H}$ & -4.93010400 & -2.43027100 & -1.96475800 \\
\hline $\mathrm{N}$ & -2.79815100 & -1.40719700 & -0.38394900 & $\mathrm{H}$ & -5.91216700 & -0.99158600 & -1.72243600 \\
\hline $\mathrm{C}$ & -0.82235400 & 3.02463200 & 1.04439700 & $\mathrm{H}$ & -4.34307300 & -0.85695900 & -2.52913900 \\
\hline $\mathrm{C}$ & -2.82596300 & 2.71242000 & -0.51617200 & $\mathrm{H}$ & -4.92254800 & -2.93598100 & 0.60177600 \\
\hline $\mathrm{C}$ & -4.24460100 & -1.10700600 & -0.36839000 & $\mathrm{H}$ & -4.48956000 & -1.63272000 & 1.72583300 \\
\hline $\mathrm{C}$ & -2.33516400 & -2.78371400 & -0.67562600 & $\mathrm{H}$ & -5.99885800 & -1.55597700 & 0.80323400 \\
\hline $\mathrm{H}$ & -0.15318800 & 3.43976300 & 0.28400000 & $\mathrm{H}$ & -1.09985500 & -3.82087300 & -2.10644400 \\
\hline $\mathrm{C}$ & 0.00223200 & 2.27984300 & 2.08817400 & $\mathrm{H}$ & -1.80228100 & -2.31895100 & -2.72591100 \\
\hline $\mathrm{C}$ & -1.57576900 & 4.16112100 & 1.73577400 & $\mathrm{H}$ & -0.43289800 & -2.26405100 & -1.60466300 \\
\hline $\mathrm{C}$ & -4.18151900 & 2.78331100 & 0.18019800 & $\mathrm{H}$ & -0.83634100 & -3.02189100 & 0.89789600 \\
\hline $\mathrm{H}$ & -2.47413100 & 3.73066300 & -0.69017700 & $\mathrm{H}$ & -2.48970000 & -3.42401000 & 1.39588800 \\
\hline $\mathrm{C}$ & -2.89022800 & 2.04402100 & -1.88637900 & $\mathrm{H}$ & -1.57809600 & -4.52150800 & 0.35062000 \\
\hline
\end{tabular}

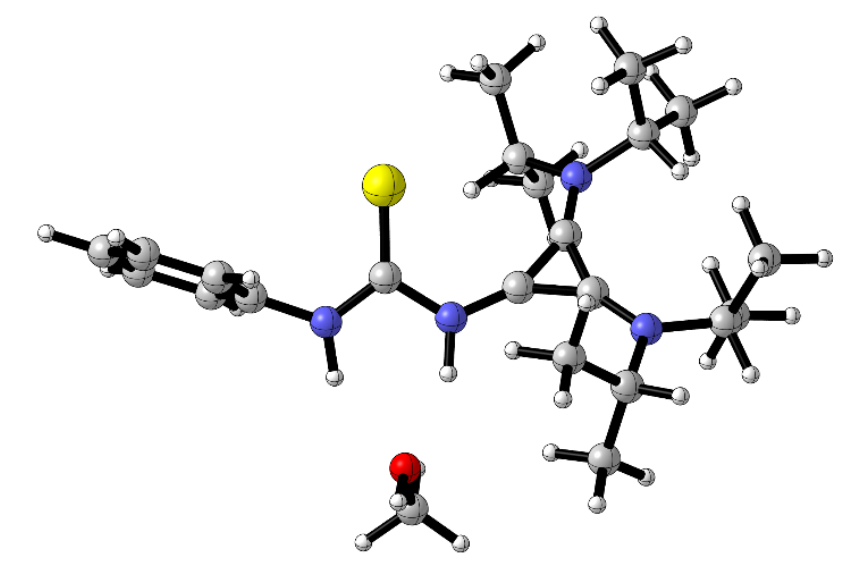

Figure S68. Optimized Higher Energy Structure of Z,Z-1-INT 1 (3).

- Thermochemistry -

(0 imaginary frequencies) 
Zero-point correction=

Thermal correction to Energy=

Thermal correction to Enthalpy=

Thermal correction to Gibbs Free Energy=

Sum of electronic and zero-point Energies=

Sum of electronic and thermal Energies=

Sum of electronic and thermal Enthalpies=

Sum of electronic and thermal Free Energies=
0.602102 (Hartree/Particle)

0.637039

0.637983

0.532983

$-1591.461183$

$-1591.426246$

$-1591.425302$

$-1591.530302$

$\begin{array}{lrrr}\mathrm{C} & -1.79059800 & 0.00961900 & -0.17782400 \\ \mathrm{~S} & -1.62128000 & -1.42415200 & -0.98901700 \\ \mathrm{~N} & -2.95112000 & 0.59659600 & 0.15617500 \\ \mathrm{H} & -2.91311000 & 1.48259600 & 0.64946300 \\ \mathrm{~N} & -0.69418900 & 0.78944000 & 0.21489900 \\ \mathrm{H} & -0.87706400 & 1.75361600 & 0.49438100 \\ \mathrm{O} & -1.85134100 & 3.15915500 & 1.27740500 \\ \mathrm{H} & -2.07852100 & 3.99199600 & 0.86050500 \\ \mathrm{C} & -1.76420500 & 3.34082400 & 2.69352700 \\ \mathrm{H} & -2.70927200 & 3.70559200 & 3.10190700 \\ \mathrm{H} & -1.54940000 & 2.36201300 & 3.12100300 \\ \mathrm{H} & -0.95758900 & 4.03091100 & 2.95415300 \\ \mathrm{C} & -4.24318800 & 0.04733700 & -0.12636200 \\ \mathrm{C} & -4.92760100 & -0.61739400 & 0.88159300 \\ \mathrm{C} & -4.80892300 & 0.21274100 & -1.38339800 \\ \mathrm{C} & -6.19605200 & -1.12246300 & 0.62854700 \\ \mathrm{H} & -4.46086500 & -0.74578400 & 1.85139500 \\ \mathrm{C} & -6.07486600 & -0.29895800 & -1.63212400 \\ \mathrm{H} & -4.25222600 & 0.72469200 & -2.15936200 \\ \mathrm{C} & -6.76849600 & -0.96389800 & -0.62738400 \\ \mathrm{H} & -7.75629300 & -1.36285600 & -0.82501900 \\ \mathrm{H} & -6.73413800 & -1.64412900 & 1.41089700 \\ \mathrm{H} & -6.52072600 & -0.17855900 & -2.61209900 \\ \mathrm{C} & 0.59164000 & 0.40887800 & 0.07663900 \\ \mathrm{C} & 1.58334600 & -0.54320900 & 0.10618600 \\ \mathrm{C} & 1.88353900 & 0.80862400 & -0.15738700 \\ \mathrm{~N} & 1.96792200 & -1.78765700 & 0.27935000 \\ \mathrm{~N} & 2.71023100 & 1.79711100 & -0.43525500 \\ \mathrm{C} & 1.02792400 & -2.69421000 & 0.99683700 \\ \mathrm{C} & 3.25776000 & -2.19897900 & -0.32640700 \\ \mathrm{C} & 4.16784300 & 1.71914900 & -0.18835100 \\ \mathrm{C} & 2.15912000 & 3.09460800 & -0.88964700 \\ \mathrm{H} & 0.08399400 & -2.14846100 & 0.98931800 \\ \mathrm{C} & 1.44445400 & -2.89141700 & 2.45402800\end{array}$

$\begin{array}{lrrr}\mathrm{C} & 0.76982100 & -4.00680200 & 0.27145600 \\ \mathrm{C} & 3.06642100 & -2.82732800 & -1.70710600 \\ \mathrm{H} & 3.78595400 & -1.25823600 & -0.48263100 \\ \mathrm{C} & 4.12279400 & -3.04882200 & 0.59493300 \\ \mathrm{H} & 4.46877600 & 2.76057200 & -0.04380800 \\ \mathrm{C} & 4.49309200 & 0.98902200 & 1.11037600 \\ \mathrm{C} & 4.90766700 & 1.18435400 & -1.41084400 \\ \mathrm{H} & 3.01390400 & 3.61376900 & -1.33004800 \\ \mathrm{C} & 1.11680600 & 2.91998900 & -1.98840800 \\ \mathrm{C} & 1.64782100 & 3.91502300 & 0.29238300 \\ \mathrm{H} & 2.32704500 & -3.52378200 & 2.55519300 \\ \mathrm{H} & 1.64682600 & -1.93198300 & 2.93665500 \\ \mathrm{H} & 0.62709700 & -3.37497100 & 2.99407900 \\ \mathrm{H} & 0.44433500 & -3.82882600 & -0.75393200 \\ \mathrm{H} & 1.64138400 & -4.66450200 & 0.26550900 \\ \mathrm{H} & -0.03403400 & -4.53350800 & 0.78999300 \\ \mathrm{H} & 2.64229100 & -3.82943300 & -1.64845200 \\ \mathrm{H} & 2.41044400 & -2.21157800 & -2.32675000 \\ \mathrm{H} & 4.03659900 & -2.90414600 & -2.20450500 \\ \mathrm{H} & 4.27466600 & -2.56522700 & 1.56194600 \\ \mathrm{H} & 3.69719600 & -4.03919600 & 0.76243800 \\ \mathrm{H} & 5.09921400 & -3.18875700 & 0.12642000 \\ \mathrm{H} & 4.22824300 & -0.06888700 & 1.08189300 \\ \mathrm{H} & 5.56615000 & 1.05172800 & 1.29999200 \\ \mathrm{H} & 3.97220300 & 1.44970900 & 1.95287100 \\ \mathrm{H} & 4.63308200 & 0.14957100 & -1.62851200 \\ \mathrm{H} & 4.67764800 & 1.78372300 & -2.29477000 \\ \mathrm{H} & 5.98664800 & 1.22210700 & -1.24666300 \\ \mathrm{H} & 0.86090500 & 3.89703400 & -2.40296200 \\ \mathrm{H} & 1.50319400 & 2.29774200 & -2.79788100 \\ \mathrm{H} & 0.19450100 & 2.46675800 & -1.61763300 \\ \mathrm{H} & 0.76358700 & 3.45090500 & 0.74038400 \\ \mathrm{H} & 2.41481400 & 4.01460800 & 1.06394400 \\ \mathrm{H} & 1.36711100 & 4.91782000 & -0.03803700\end{array}$




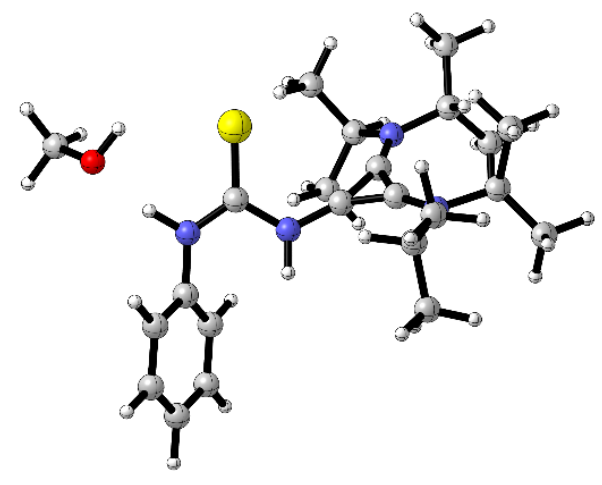

Figure S69. Optimized Higher Energy Structure of E,Z-1-Cy-INT 1 (1).

- Thermochemistry -

(0 imaginary frequencies)

Zero-point correction $=$

Thermal correction to Energy=

0.602876 (Hartree/Particle)

Thermal correction to Enthalpy=

0.637412

0.638357

Thermal correction to Gibbs Free Energy $=\quad 0.533917$

Sum of electronic and zero-point Energies $=\quad-1591.458339$

Sum of electronic and thermal Energies $=\quad-1591.423802$

Sum of electronic and thermal Enthalpies $=\quad-1591.422858$

Sum of electronic and thermal Free Energies $=\quad-1591.527297$

\begin{tabular}{|c|c|c|c|c|c|c|c|}
\hline $\mathrm{C}$ & -1.79693300 & -0.52214200 & -0.61005500 & $\mathrm{C}$ & 1.56843300 & 2.87362600 & -0.96466700 \\
\hline $\mathrm{S}$ & -1.23325300 & -1.63392800 & -1.72753600 & $\mathrm{C}$ & 3.63073000 & -1.88669000 & 0.51628900 \\
\hline $\mathrm{N}$ & -3.09178800 & -0.27257500 & -0.39658100 & $\mathrm{C}$ & 1.41434900 & -2.90950100 & 1.30977300 \\
\hline $\mathrm{H}$ & -3.73570700 & -0.71657100 & -1.07091200 & $\mathrm{C}$ & 4.52489100 & 1.26908900 & -1.59678900 \\
\hline $\mathrm{N}$ & -0.91556300 & 0.24652400 & 0.13616600 & $\mathrm{H}$ & 4.00161600 & 0.88146800 & 0.43667500 \\
\hline $\mathrm{C}$ & -3.64118800 & 0.66998100 & 0.51738300 & $\mathrm{C}$ & 4.42138400 & 2.94235100 & 0.31132700 \\
\hline $\mathrm{C}$ & -4.51859100 & 1.63604600 & 0.03148600 & $\mathrm{C}$ & 2.13593800 & 3.43969400 & -2.26091500 \\
\hline $\mathrm{C}$ & -3.35503700 & 0.60085600 & 1.88024300 & $\mathrm{H}$ & 3.90312700 & -1.10568400 & -0.19492500 \\
\hline $\mathrm{C}$ & -5.09704400 & 2.54150800 & 0.91056900 & $\mathrm{C}$ & 4.28649300 & -1.58506600 & 1.86168700 \\
\hline $\mathrm{H}$ & -4.74458100 & 1.66497400 & -1.02834000 & $\mathrm{C}$ & 4.09786800 & -3.20985500 & -0.07841300 \\
\hline $\mathrm{C}$ & -3.92495600 & 1.52276800 & 2.74876000 & $\mathrm{H}$ & 2.17204800 & -3.39991000 & 1.92551600 \\
\hline $\mathrm{H}$ & -2.70990600 & -0.18512200 & 2.25634300 & $\mathrm{C}$ & 0.34583900 & -2.35922200 & 2.24876700 \\
\hline $\mathrm{C}$ & -4.79543700 & 2.49296500 & 2.26669200 & $\mathrm{C}$ & 0.84281300 & -3.92374000 & 0.32563700 \\
\hline $\mathrm{H}$ & -5.24748000 & 3.20260600 & 2.94898000 & $\mathrm{H}$ & 4.05990700 & 0.38104700 & -2.03288400 \\
\hline $\mathrm{H}$ & -1.26653700 & 1.09916700 & 0.55483100 & $\mathrm{H}$ & 4.49510400 & 2.06647400 & -2.33987600 \\
\hline $\mathrm{C}$ & -5.23525600 & -2.72617800 & -2.06106300 & $\mathrm{H}$ & 5.57464400 & 1.04174900 & -1.39604000 \\
\hline $\mathrm{H}$ & -5.54098700 & -3.27026400 & -2.95881600 & $\mathrm{H}$ & 4.34600400 & 3.80016000 & -0.35822200 \\
\hline $\mathrm{H}$ & -6.12948200 & -2.43340400 & -1.51189100 & $\mathrm{H}$ & 3.94258400 & 3.18960100 & 1.25998900 \\
\hline $\mathrm{H}$ & -4.62438600 & -3.38056900 & -1.42978800 & $\mathrm{H}$ & 5.48288700 & 2.77272700 & 0.50230300 \\
\hline $\mathrm{O}$ & -4.54214700 & -1.52950200 & -2.39983800 & $\mathrm{H}$ & 3.09445200 & 3.93932300 & -2.11259900 \\
\hline $\mathrm{H}$ & -3.73151000 & -1.76987900 & -2.86032800 & $\mathrm{H}$ & 2.25465200 & 2.66044800 & -3.01540200 \\
\hline $\mathrm{C}$ & 0.43767400 & 0.07726200 & 0.08691200 & $\mathrm{H}$ & 1.43756200 & 4.18311600 & -2.65033600 \\
\hline $\mathrm{C}$ & 1.65793000 & 0.65741400 & -0.11441000 & $\mathrm{H}$ & 4.06483800 & -2.36125300 & 2.59831400 \\
\hline $\mathrm{C}$ & 1.55462100 & -0.69647400 & 0.29655000 & $\mathrm{H}$ & 5.37156500 & -1.54025700 & 1.74652900 \\
\hline $\mathrm{N}$ & 2.35520200 & 1.72100300 & -0.43936700 & $\mathrm{H}$ & 3.94413000 & -0.62802200 & 2.26497700 \\
\hline $\mathrm{N}$ & 2.15239800 & -1.81236200 & 0.62462500 & $\mathrm{H}$ & 3.87842300 & -4.05582000 & 0.57675900 \\
\hline $\mathrm{C}$ & 3.83335800 & 1.67368000 & -0.29568700 & $\mathrm{H}$ & 3.63770200 & -3.38919000 & -1.05132700 \\
\hline
\end{tabular}




$\begin{array}{rrrrrrrr}\mathrm{H} & 5.18044800 & -3.17224400 & -0.21430600 & \mathrm{H} & -5.78574300 & 3.28801100 & 0.53351200 \\ \mathrm{H} & -0.04746300 & -3.17619200 & 2.85601100 & \mathrm{H} & -3.70749000 & 1.46543100 & 3.80870900 \\ \mathrm{H} & 0.75433100 & -1.60212300 & 2.92303100 & \mathrm{H} & 0.59981000 & 2.43310400 & -1.21547000 \\ \mathrm{H} & -0.49403900 & -1.93179000 & 1.69553800 & \mathrm{C} & 1.32779200 & 3.94392700 & 0.09864300 \\ \mathrm{H} & 0.07602700 & -3.45909000 & -0.29589700 & \mathrm{H} & 2.22936600 & 4.51400200 & 0.32235900 \\ \mathrm{H} & 1.61396800 & -4.33581400 & -0.32493400 & \mathrm{H} & 0.57296200 & 4.64652100 & -0.26181800 \\ \mathrm{H} & 0.38964800 & -4.74950700 & 0.87927100 & \mathrm{H} & 0.96094700 & 3.49969300 & 1.02752500\end{array}$

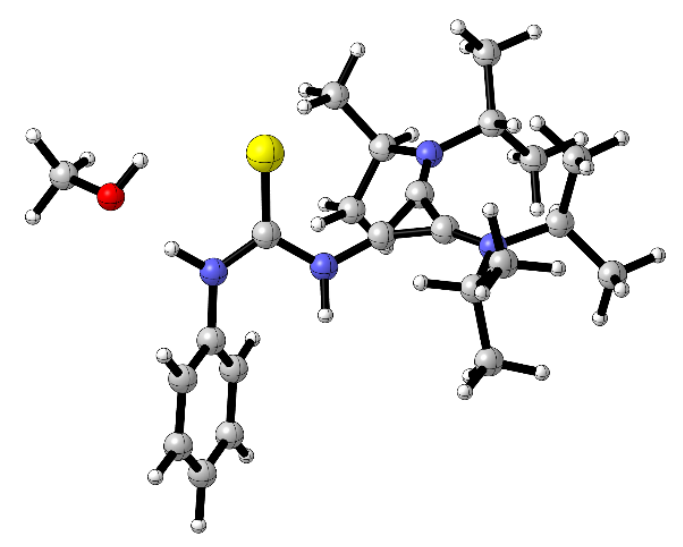

Figure S70. Optimized Higher Energy Structure of E,Z-1-Cy-INT 1 (2).

- Thermochemistry -

\section{(0 imaginary frequencies)}

Zero-point correction= Thermal correction to Energy= Thermal correction to Enthalpy= Thermal correction to Gibbs Free Energy= Sum of electronic and zero-point Energies= Sum of electronic and thermal Energies= Sum of electronic and thermal Enthalpies= Sum of electronic and thermal Free Energies=
0.602864 (Hartree/Particle)

0.637406

0.638350

0.533873

$-1591.458350$

$-1591.423808$

$-1591.422864$

$-1591.527341$

$\begin{array}{lrrr}\mathrm{C} & -1.79719900 & 0.52238800 & 0.60993700 \\ \mathrm{~S} & -1.23368300 & 1.63446600 & 1.72721100 \\ \mathrm{~N} & -3.09201000 & 0.27275900 & 0.39628400 \\ \mathrm{H} & -3.73606100 & 0.71683400 & 1.07043700 \\ \mathrm{~N} & -0.91571300 & -0.24643100 & -0.13598100 \\ \mathrm{C} & -3.64102900 & -0.67022500 & -0.51749100 \\ \mathrm{C} & -4.51756400 & -1.63694300 & -0.03133600 \\ \mathrm{C} & -3.35524800 & -0.60091100 & -1.88041100 \\ \mathrm{C} & -5.09561000 & -2.54281700 & -0.91026700 \\ \mathrm{H} & -4.74318100 & -1.66606500 & 1.02856500 \\ \mathrm{C} & -3.92472900 & -1.52323400 & -2.74877900 \\ \mathrm{H} & -2.71072400 & 0.18550300 & -2.25664300 \\ \mathrm{C} & -4.79441300 & -2.49403300 & -2.26647200\end{array}$

$\begin{array}{lrrr}\mathrm{H} & -5.24614600 & -3.20398500 & -2.94864500 \\ \mathrm{H} & -1.26659800 & -1.09918500 & -0.55449500 \\ \mathrm{C} & -5.23508300 & 2.72682700 & 2.06116500 \\ \mathrm{H} & -5.54021900 & 3.27097000 & 2.95908800 \\ \mathrm{H} & -6.12967100 & 2.43418600 & 1.51251200 \\ \mathrm{H} & -4.62448700 & 3.38113900 & 1.42954400 \\ \mathrm{O} & -4.54195400 & 1.53006200 & 2.39956100 \\ \mathrm{H} & -3.73092600 & 1.77036500 & 2.85942600 \\ \mathrm{C} & 0.43751500 & -0.07711400 & -0.08655400 \\ \mathrm{C} & 1.65777600 & -0.65728400 & 0.11475800 \\ \mathrm{C} & 1.55449300 & 0.69654300 & -0.29634900 \\ \mathrm{~N} & 2.35509700 & -1.72087100 & 0.43964100 \\ \mathrm{~N} & 2.15223900 & 1.81236900 & -0.62466100\end{array}$




$\begin{array}{rrrrrrr}3.83323100 & -1.67355200 & 0.29574700 & \mathrm{H} & 2.25486200 & -2.66032900 & 3.01567400 \\ 1.56838700 & -2.87349300 & 0.96502500 & \mathrm{H} & 1.43768700 & -4.18297900 & 2.65071000 \\ 3.63056000 & 1.88679300 & -0.51623300 & \mathrm{H} & 4.06478400 & 2.36116500 & -2.59826900 \\ 1.41415100 & 2.90931600 & -1.31007600 & \mathrm{H} & 5.37149200 & 1.54030500 & -1.74632100 \\ 4.52497300 & -1.26908100 & 1.59677800 & \mathrm{H} & 3.94412800 & 0.62796000 & -2.26477700 \\ 4.00140100 & -0.88129600 & -0.43659100 & \mathrm{H} & 3.87810600 & 4.05592800 & -0.57695600 \\ 4.42112800 & -2.94222000 & -0.31139600 & \mathrm{H} & 3.63735200 & 3.38948500 & 1.05120300 \\ 2.13603300 & -3.43957200 & 2.26120200 & \mathrm{H} & 5.18015600 & 3.17254200 & 0.21428400 \\ 3.90295000 & 1.10588900 & 0.19510300 & \mathrm{H} & -0.04749800 & 3.17560000 & -2.85654500 \\ 4.28642700 & 1.58505600 & -1.86155600 & \mathrm{H} & 0.75443100 & 1.60158000 & -2.92317200 \\ 4.09758000 & 3.21006100 & 0.07833400 & \mathrm{H} & -0.49410000 & 1.93139300 & -1.69587100 \\ 2.17188900 & 3.39969900 & -1.92579300 & \mathrm{H} & 0.07525500 & 3.45925400 & 0.29506500 \\ 0.34580400 & 2.35877700 & -2.24910200 & \mathrm{H} & 1.61332700 & 4.33567900 & 0.32466200 \\ 0.84238100 & 3.92370200 & -0.32621300 & \mathrm{H} & 0.38959900 & 4.74951900 & -0.88008900 \\ 4.06010100 & -0.38104600 & 2.03300500 & \mathrm{H} & -5.78369000 & -3.28980400 & -0.53303900 \\ 4.49524800 & -2.06652300 & 2.33980700 & \mathrm{H} & -3.70757700 & -1.46575200 & -3.80878400 \\ 5.57470900 & -1.04178400 & 1.39589100 & \mathrm{H} & 0.59979300 & -2.43296500 & 1.21593400 \\ 4.34578600 & -3.80004900 & 0.35813500 & \mathrm{C} & 1.32762100 & -3.94379500 & -0.09825700 \\ 3.94219300 & -3.18941900 & -1.26000500 & \mathrm{H} & 2.22915200 & -4.51392200 & -0.32201900 \\ 5.48261400 & -2.77263800 & -0.50249900 & \mathrm{H} & 0.57277400 & -4.64634100 & 0.26226300 \\ 3.09451700 & -3.93922400 & 2.11276000 & \mathrm{H} & 0.96073800 & -3.49956000 & -1.02712500\end{array}$

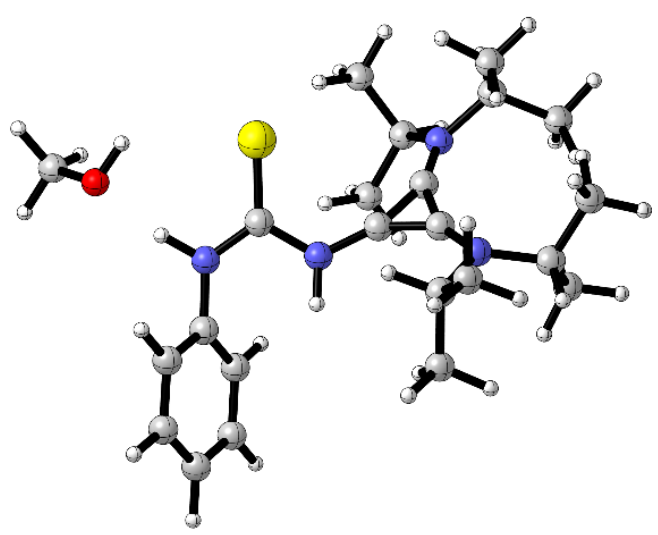

Figure S71. Optimized Higher Energy Structure of E,Z-1-Cy-INT 1 (3).

- Thermochemistry -

(0 imaginary frequencies)

Zero-point correction $=$

Thermal correction to Energy=

0.601940 (Hartree/Particle)

Thermal correction to Enthalpy=

0.636917

Thermal correction to Gibbs Free Energy=
Sum of electronic and zero-point Energies=

0.637861

0.531739

Sum of electronic and thermal Energies=

$-1591.456368$

Sum of electronic and thermal Enthalpies=

$-1591.421391$

Sum of electronic and thermal Free Energies=

$-1591.420447$

$-1591.526569$ 


\begin{tabular}{|c|c|c|c|c|c|c|c|}
\hline $\mathrm{C}$ & -1.72335200 & -0.76920800 & -0.41042200 & $\mathrm{H}$ & 2.43907900 & -3.11721400 & 2.19246000 \\
\hline $\mathrm{S}$ & -1.19392600 & -2.09765200 & -1.27394300 & $\mathrm{C}$ & 0.50970300 & -2.23524800 & 2.33330700 \\
\hline $\mathrm{N}$ & -3.01051100 & -0.46961600 & -0.21660100 & $\mathrm{C}$ & 1.19280000 & -4.06164600 & 0.72385900 \\
\hline $\mathrm{H}$ & -3.67373200 & -1.04132300 & -0.76493900 & $\mathrm{H}$ & 3.70940900 & -3.23862800 & 0.27785200 \\
\hline $\mathrm{N}$ & -0.82331300 & 0.15010800 & 0.11967900 & $\mathrm{C}$ & 3.78344400 & -1.88351800 & -1.34838900 \\
\hline $\mathrm{C}$ & -3.52672100 & 0.69022100 & 0.42834500 & $\mathrm{C}$ & 4.74571300 & -1.54191200 & 1.00823100 \\
\hline $\mathrm{C}$ & -4.37483400 & 1.52691400 & -0.29267400 & $\mathrm{H}$ & 0.30636200 & 3.21282900 & 0.73478000 \\
\hline $\mathrm{C}$ & -3.23210000 & 0.96488900 & 1.76296300 & $\mathrm{H}$ & 1.53757900 & 4.38571700 & 0.24750900 \\
\hline $\mathrm{C}$ & -4.91747100 & 2.64735800 & 0.32113800 & $\mathrm{H}$ & -0.02950400 & 4.37331200 & -0.55893400 \\
\hline $\mathrm{H}$ & -4.60648800 & 1.28833100 & -1.32440900 & $\mathrm{H}$ & 2.67089600 & 4.11303100 & -2.13692500 \\
\hline $\mathrm{C}$ & -3.76716800 & 2.09824300 & 2.36277500 & $\mathrm{H}$ & 2.21313400 & 2.70405800 & -3.11589600 \\
\hline $\mathrm{H}$ & -2.61039200 & 0.28115200 & 2.33002700 & $\mathrm{H}$ & 1.07706400 & 4.03799100 & -2.87757100 \\
\hline $\mathrm{C}$ & -4.60853500 & 2.93967100 & 1.64468600 & $\mathrm{H}$ & 4.86471300 & 0.70338400 & -0.83243100 \\
\hline $\mathrm{H}$ & -5.03390500 & 3.81521800 & 2.12000500 & $\mathrm{H}$ & 4.50778500 & 1.94571900 & -2.03796200 \\
\hline $\mathrm{H}$ & -1.17987000 & 1.07184000 & 0.34350700 & $\mathrm{H}$ & 5.62052400 & 2.28907800 & -0.70638500 \\
\hline $\mathrm{C}$ & -5.32576100 & -3.10913700 & -1.35067500 & $\mathrm{H}$ & -0.37560700 & -2.03401400 & 1.72755900 \\
\hline $\mathrm{H}$ & -5.68637800 & -3.77715900 & -2.13738800 & $\mathrm{H}$ & 0.22164700 & -2.96094500 & 3.09548300 \\
\hline $\mathrm{H}$ & -6.18569200 & -2.64986000 & -0.86462100 & $\mathrm{H}$ & 0.82015200 & -1.31943300 & 2.84289700 \\
\hline $\mathrm{H}$ & -4.76311300 & -3.68927300 & -0.61160700 & $\mathrm{H}$ & 0.37581000 & -3.80834500 & 0.04822800 \\
\hline $\mathrm{O}$ & -4.54169500 & -2.05280800 & -1.89597600 & $\mathrm{H}$ & 2.00696600 & -4.49675200 & 0.14058500 \\
\hline $\mathrm{H}$ & -3.76603500 & -2.43379000 & -2.31938700 & $\mathrm{H}$ & 0.84625700 & -4.82435000 & 1.42472700 \\
\hline $\mathrm{C}$ & 0.52283200 & 0.00555000 & 0.06985800 & $\mathrm{H}$ & 4.77581900 & -2.18015400 & -1.69345800 \\
\hline $\mathrm{C}$ & 1.70496600 & 0.64931500 & -0.16016000 & $\mathrm{H}$ & 3.03740900 & -2.45304800 & -1.90657000 \\
\hline $\mathrm{C}$ & 1.68512200 & -0.70579900 & 0.27251800 & $\mathrm{H}$ & 3.65028300 & -0.82699400 & -1.58941700 \\
\hline $\mathrm{N}$ & 2.22717800 & 1.78810300 & -0.57612200 & $\mathrm{H}$ & 4.81296000 & -0.46265700 & 0.88016900 \\
\hline $\mathrm{N}$ & 2.28929800 & -1.81464100 & 0.62494100 & $\mathrm{H}$ & 4.57164700 & -1.75251400 & 2.06612700 \\
\hline $\mathrm{C}$ & 1.26022000 & 2.75058600 & -1.16881800 & $\mathrm{H}$ & 5.71275300 & -1.96957100 & 0.73527200 \\
\hline $\mathrm{C}$ & 3.54663800 & 2.29621800 & -0.13015100 & $\mathrm{H}$ & -5.58212100 & 3.29433500 & -0.23859200 \\
\hline $\mathrm{C}$ & 1.63883100 & -2.82798100 & 1.50200700 & $\mathrm{H}$ & -3.54557300 & 2.30887600 & 3.40227600 \\
\hline $\mathrm{C}$ & 3.65036500 & -2.15692000 & 0.14473800 & $\mathrm{H}$ & 3.48985800 & 3.37164500 & -0.30514100 \\
\hline $\mathrm{C}$ & 0.74136000 & 3.73464400 & -0.12291700 & $\mathrm{C}$ & 3.72485600 & 2.10161400 & 1.37277300 \\
\hline $\mathrm{H}$ & 0.42627700 & 2.13628900 & -1.51775000 & $\mathrm{H}$ & 3.68253900 & 1.04886800 & 1.65947100 \\
\hline $\mathrm{C}$ & 1.85085300 & 3.43840100 & -2.39433100 & $\mathrm{H}$ & 4.69276400 & 2.49442500 & 1.68982100 \\
\hline $\mathrm{C}$ & 4.69858800 & 1.77006700 & -0.97768900 & $\mathrm{H}$ & 2.94446100 & 2.63323400 & 1.92240700 \\
\hline
\end{tabular}




\section{Rotational Energy Barrier Between $E, Z$ - and $Z, Z$-Conformers}

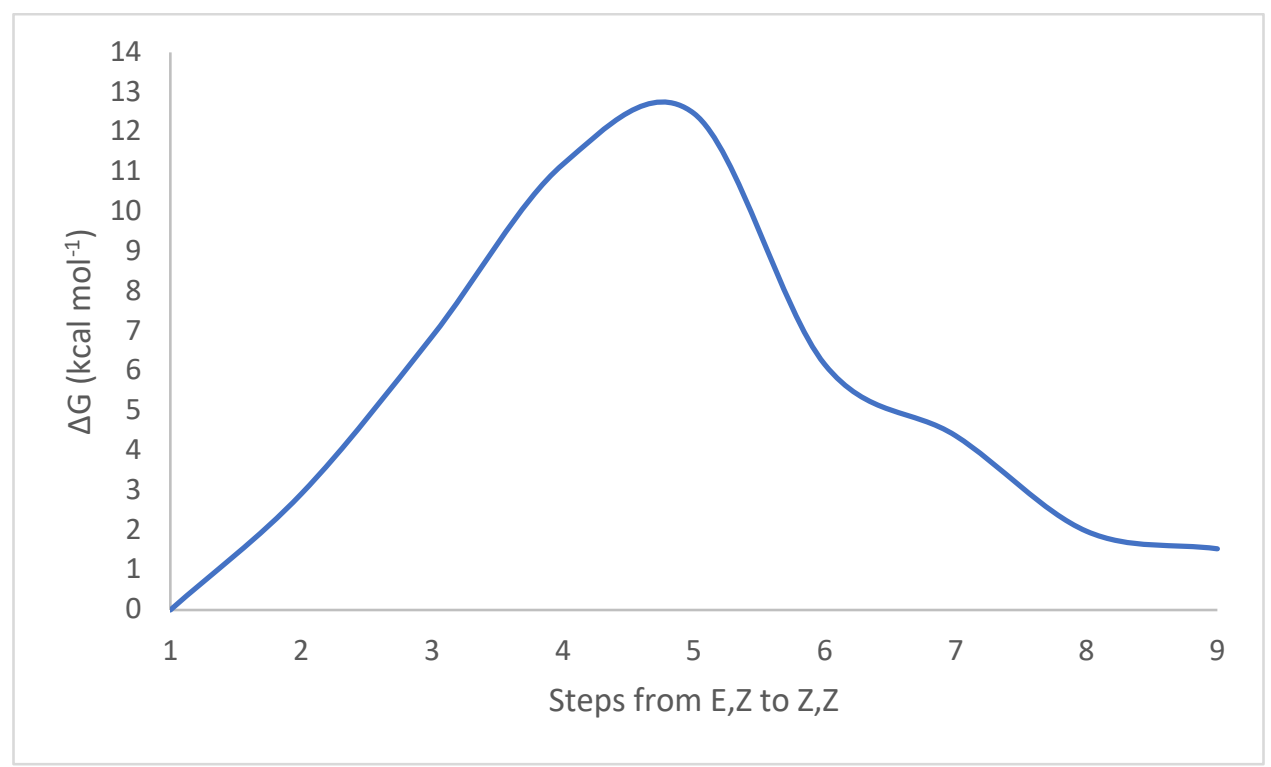

Figure S72. Plot of energy versus steps in rotation from E,Z conformer to Z,Z-conformer calculated in the absence of counterion.

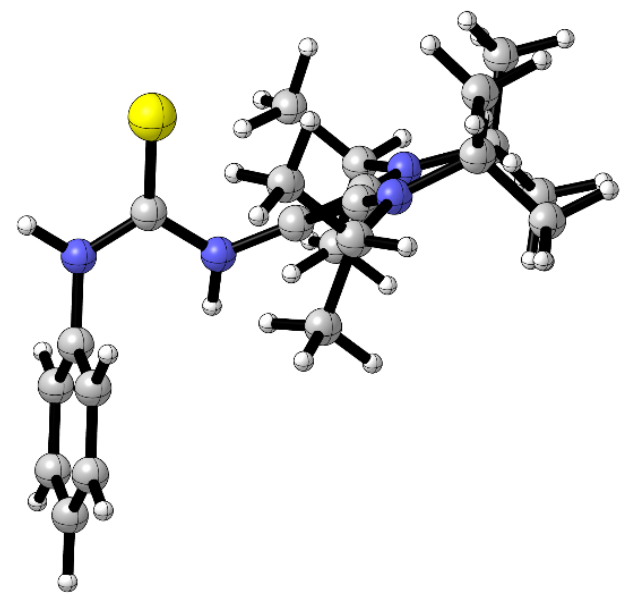

Figure S73. Optimized Structure of E,Z-Z,Z-Rotation-1

- Thermochemistry -

(0 imaginary frequencies)

Zero-point correction $=$ Thermal correction to Energy= 0.546925 (Hartree/Particle)

Thermal correction to Enthalpy=

0.577671

0.578615

Thermal correction to Gibbs Free Energy $=\quad 0.483133$ 
Sum of electronic and zero-point Energies= Sum of electronic and thermal Energies= Sum of electronic and thermal Enthalpies= Sum of electronic and thermal Free Energies=

$$
\begin{gathered}
-1475.778956 \\
-1475.748209 \\
-1475.747265 \\
-1475.842748
\end{gathered}
$$

$\begin{array}{lrrr}\mathrm{C} & -0.69315200 & -3.57749700 & -0.87163200 \\ \mathrm{H} & -3.49817500 & 1.25186900 & 0.04146900 \\ \mathrm{C} & -3.47355400 & 3.29907900 & 0.58236700 \\ \mathrm{C} & -3.40692100 & 2.37688300 & -1.78647400 \\ \mathrm{H} & -1.17860600 & 3.83322100 & -0.81862300 \\ \mathrm{C} & -0.09173900 & 3.51627300 & 1.00438600 \\ \mathrm{C} & 0.53560700 & 2.66961900 & -1.29982200 \\ \mathrm{H} & -3.25643200 & -2.22037700 & -2.66947500 \\ \mathrm{H} & -3.95866600 & -3.45572800 & -1.60771300 \\ \mathrm{H} & -4.96119400 & -2.13870500 & -2.20333600 \\ \mathrm{H} & -4.52704000 & -2.75448100 & 0.90193600 \\ \mathrm{H} & -4.31523100 & -1.05661300 & 1.35726600 \\ \mathrm{H} & -5.59268400 & -1.52599700 & 0.22163700 \\ \mathrm{H} & -0.32385000 & -2.49783600 & 1.70633000 \\ \mathrm{H} & -1.98055300 & -2.76027700 & 2.26842700 \\ \mathrm{H} & -0.95630800 & -4.13993400 & 1.84468700 \\ \mathrm{H} & -1.08128100 & -3.53157900 & -1.89077200 \\ \mathrm{H} & 0.22546200 & -2.98600300 & -0.81911000 \\ \mathrm{H} & -0.42768100 & -4.61310700 & -0.65132400 \\ \mathrm{H} & -3.04730700 & 4.26004600 & 0.28590700 \\ \mathrm{H} & -4.55941600 & 3.39134600 & 0.51886200 \\ \mathrm{H} & -3.20785900 & 3.10257700 & 1.62231500 \\ \mathrm{H} & -3.10607000 & 1.52580100 & -2.40344700 \\ \mathrm{H} & -4.48811600 & 2.50087600 & -1.87587700 \\ \mathrm{H} & -2.93706700 & 3.27559600 & -2.19441500 \\ \mathrm{H} & 0.57647300 & 4.36882700 & 0.86294200 \\ \mathrm{H} & -0.91024800 & 3.83083300 & 1.65263400 \\ \mathrm{H} & 0.45973100 & 2.72315500 & 1.51416600 \\ \mathrm{H} & 1.20736500 & 1.92585700 & -0.86292500 \\ \mathrm{H} & 0.15240600 & 2.28576100 & -2.24847800 \\ \mathrm{H} & 1.12688100 & 3.56269800 & -1.50974100\end{array}$

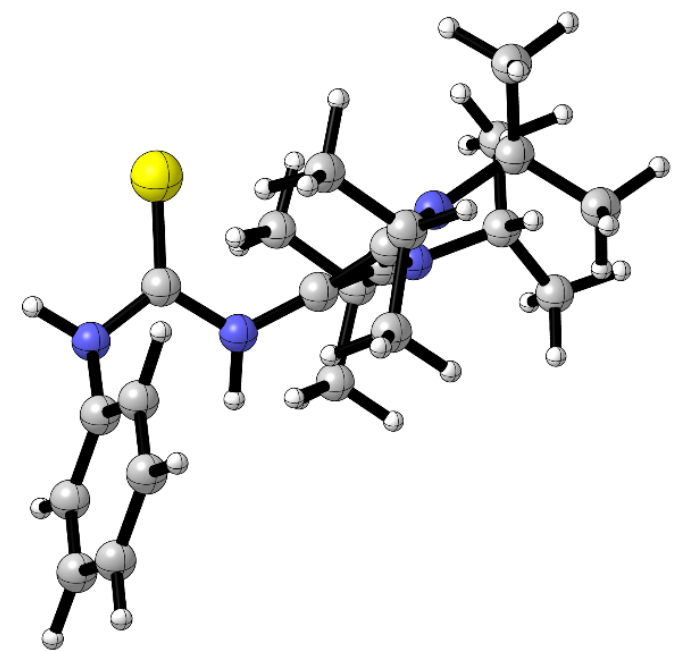

Figure S74. Optimized Structure of E,Z-Z,Z-Rotation-2

- Thermochemistry - 
(0 imaginary frequencies)

Zero-point correction=

Thermal correction to Energy=

Thermal correction to Enthalpy=

Thermal correction to Gibbs Free Energy=

Sum of electronic and zero-point Energies=

Sum of electronic and thermal Energies=

Sum of electronic and thermal Enthalpies=

Sum of electronic and thermal Free Energies=
0.547697 (Hartree/Particle)

0.578209

0.579153

0.484461

$-1475.775189$

$-1475.744677$

$-1475.743733$

$-1475.838426$

$\begin{array}{lrrr}\mathrm{C} & -1.33549700 & -3.46141700 & -1.18853800 \\ \mathrm{H} & -3.22837700 & 1.67418900 & 0.22678600 \\ \mathrm{C} & -2.78070100 & 3.57609900 & 1.04463000 \\ \mathrm{C} & -3.00738700 & 3.02352600 & -1.42857700 \\ \mathrm{H} & -0.50143600 & 3.88575900 & -0.42675600 \\ \mathrm{C} & 0.63661600 & 3.07492200 & 1.19803900 \\ \mathrm{C} & 0.89418600 & 2.51437800 & -1.26389400 \\ \mathrm{H} & -3.70274300 & -1.47875300 & -2.80343600 \\ \mathrm{H} & -4.60282100 & -2.66709600 & -1.84152600 \\ \mathrm{H} & -5.35095600 & -1.13077400 & -2.26068600 \\ \mathrm{H} & -4.98603200 & -2.15349200 & 0.72762300 \\ \mathrm{H} & -4.42159900 & -0.60193200 & 1.36928900 \\ \mathrm{H} & -5.79377000 & -0.66167900 & 0.24878700 \\ \mathrm{H} & -0.86871500 & -2.68742500 & 1.49070300 \\ \mathrm{H} & -2.56001000 & -2.76217100 & 2.00381900 \\ \mathrm{H} & -1.73359400 & -4.22660000 & 1.45107200 \\ \mathrm{H} & -1.68368900 & -3.25443700 & -2.20229000 \\ \mathrm{H} & -0.33187900 & -3.04459900 & -1.06720800 \\ \mathrm{H} & -1.25420400 & -4.54289700 & -1.06610200 \\ \mathrm{H} & -2.21078700 & 4.48543800 & 0.84121600 \\ \mathrm{H} & -3.83427000 & 3.85701300 & 1.09711100 \\ \mathrm{H} & -2.48364900 & 3.18471700 & 2.01911600 \\ \mathrm{H} & -2.91381600 & 2.23206800 & -2.17677700 \\ \mathrm{H} & -4.04521300 & 3.36277200 & -1.41461800 \\ \mathrm{H} & -2.38861800 & 3.86732600 & -1.74509900 \\ \mathrm{H} & 1.44417600 & 3.80587200 & 1.11532200 \\ \mathrm{H} & -0.05413200 & 3.41808100 & 1.96921000 \\ \mathrm{H} & 1.06053200 & 2.12339800 & 1.52703300 \\ \mathrm{H} & 1.41807400 & 1.58445800 & -1.02727700 \\ \mathrm{H} & 0.36714600 & 2.39265200 & -2.21301500 \\ \mathrm{H} & 1.65257900 & 3.28872800 & -1.39426300\end{array}$




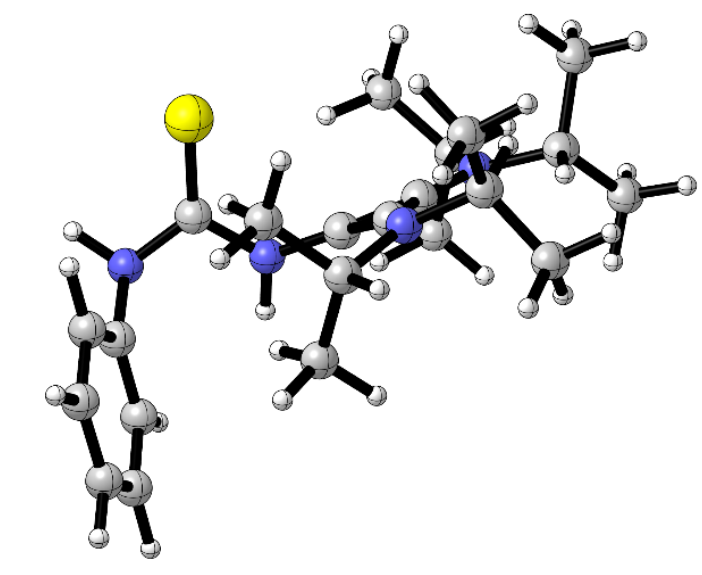

Figure S75. Optimized Structure of E,Z-Z,Z-Rotation-3

- Thermochemistry -

(0 imaginary frequencies)

Zero-point correction=

Thermal correction to Energy=

0.548082 (Hartree/Particle)

Thermal correction to Enthalpy=

0.578388

Thermal correction to Gibbs Free Energy=
Sum of electronic and zero-point Energies

0.579332

Sum of electronic and zero-point Energies=
Sum of electronic and thermal Energies=

0.486199

Sum of electronic and thermal Enthalpies=

$-1475.770696$

$-1475.740391$

$-1475.739447$

Sum of electronic and thermal Free Energies $=$

$-1475.832580$

$\begin{array}{lrrr}\mathrm{C} & 1.52520500 & -1.64898200 & 1.04202700 \\ \mathrm{~S} & 0.99796600 & -1.24994800 & 2.55160700 \\ \mathrm{~N} & 0.76310400 & -1.45167100 & -0.08701600 \\ \mathrm{H} & 1.08019300 & -1.85711200 & -0.95671600 \\ \mathrm{~N} & 2.78284500 & -2.13147400 & 0.73341700 \\ \mathrm{C} & 3.62469000 & -1.24417600 & -0.04189100 \\ \mathrm{C} & 3.80691200 & -1.47501600 & -1.39878900 \\ \mathrm{C} & 4.22504800 & -0.15174400 & 0.57655300 \\ \mathrm{C} & 4.57586100 & -0.59370700 & -2.15040800 \\ \mathrm{H} & 3.37012400 & -2.35761800 & -1.85361100 \\ \mathrm{C} & 5.00617300 & 0.71564200 & -0.17569000 \\ \mathrm{H} & 4.07669000 & 0.01383300 & 1.63832600 \\ \mathrm{C} & 5.17487200 & 0.50106600 & -1.53939300 \\ \mathrm{H} & 5.78710300 & 1.17798300 & -2.12326000 \\ \mathrm{H} & 3.24480700 & -2.44063700 & 1.57959000 \\ \mathrm{H} & 5.48588600 & 1.56010600 & 0.30524000 \\ \mathrm{H} & 4.72414300 & -0.77469800 & -3.20825700 \\ \mathrm{C} & -0.32087700 & -0.60762900 & -0.10619200 \\ \mathrm{C} & -1.67369600 & -0.43569100 & -0.20931500 \\ \mathrm{C} & -0.79415300 & 0.67580700 & -0.03084000 \\ \mathrm{~N} & -2.87087900 & -0.93068900 & -0.36001300 \\ \mathrm{~N} & -0.60843800 & 1.95899800 & 0.10092100 \\ \mathrm{C} & -4.04688900 & -0.04857500 & -0.53819000 \\ \mathrm{C} & -3.04123600 & -2.40706400 & -0.29710200 \\ \mathrm{C} & -1.77363100 & 2.87020000 & 0.21125000 \\ \mathrm{C} & 0.77069800 & 2.51770300 & 0.01275100\end{array}$

$\begin{array}{lrrr}\text { C } & -4.81048600 & -0.39851500 & -1.81193200 \\ \mathrm{H} & -3.63290800 & 0.95237200 & -0.67246300 \\ \mathrm{C} & -4.92498300 & -0.05973200 & 0.70802100 \\ \mathrm{C} & -2.56778100 & -2.95175900 & 1.04589400 \\ \mathrm{H} & -4.11653700 & -2.57071800 & -0.37507100 \\ \mathrm{C} & -2.36323300 & -3.08101900 & -1.48501300 \\ \mathrm{H} & -2.61149500 & 2.22672600 & 0.48708200 \\ \mathrm{C} & -1.59518700 & 3.87739900 & 1.34189200 \\ \mathrm{C} & -2.06691400 & 3.52848800 & -1.13285900 \\ \mathrm{H} & 0.62221700 & 3.57696200 & -0.20443600 \\ \mathrm{C} & 1.51147700 & 2.38088100 & 1.33723700 \\ \mathrm{C} & 1.54288400 & 1.90666300 & -1.15215800 \\ \mathrm{H} & -4.14930500 & -0.39375100 & -2.68098100 \\ \mathrm{H} & -5.29466800 & -1.37551100 & -1.74647000 \\ \mathrm{H} & -5.59505600 & 0.34269500 & -1.97563700 \\ \mathrm{H} & -5.35966600 & -1.04789200 & 0.87993800 \\ \mathrm{H} & -4.35293300 & 0.22254700 & 1.59500800 \\ \mathrm{H} & -5.74900100 & 0.64668800 & 0.58902600 \\ \mathrm{H} & -1.49121200 & -2.81819600 & 1.18186000 \\ \mathrm{H} & -3.07921300 & -2.45570600 & 1.87315200 \\ \mathrm{H} & -2.78007700 & -4.02104600 & 1.10369200 \\ \mathrm{H} & -2.72072500 & -2.66959100 & -2.43089900 \\ \mathrm{H} & -1.27724100 & -2.96142100 & -1.43520500 \\ \mathrm{H} & -2.57390100 & -4.15188700 & -1.47342100 \\ \mathrm{H} & -0.78819800 & 4.58554900 & 1.14139000 \\ \mathrm{H} & -2.51582900 & 4.45385200 & 1.44985900\end{array}$




$\begin{array}{lrrrrrrr}\mathrm{H} & -1.39563900 & 3.37477500 & 2.28976300 & \mathrm{H} & 0.97711600 & 2.87354600 & 2.15056000 \\ \mathrm{H} & -2.23498900 & 2.77847300 & -1.91028000 & \mathrm{H} & 1.64307500 & 1.32940600 & 1.60053300 \\ \mathrm{H} & -2.95980900 & 4.15265400 & -1.05856200 & \mathrm{H} & 1.80665000 & 0.86299700 & -0.96370900 \\ \mathrm{H} & -1.24038500 & 4.16976900 & -1.44979600 & \mathrm{H} & 0.97622400 & 1.97322000 & -2.08398400 \\ \mathrm{H} & 2.50044600 & 2.83637300 & 1.25026500 & \mathrm{H} & 2.48172800 & 2.44682600 & -1.28451100\end{array}$

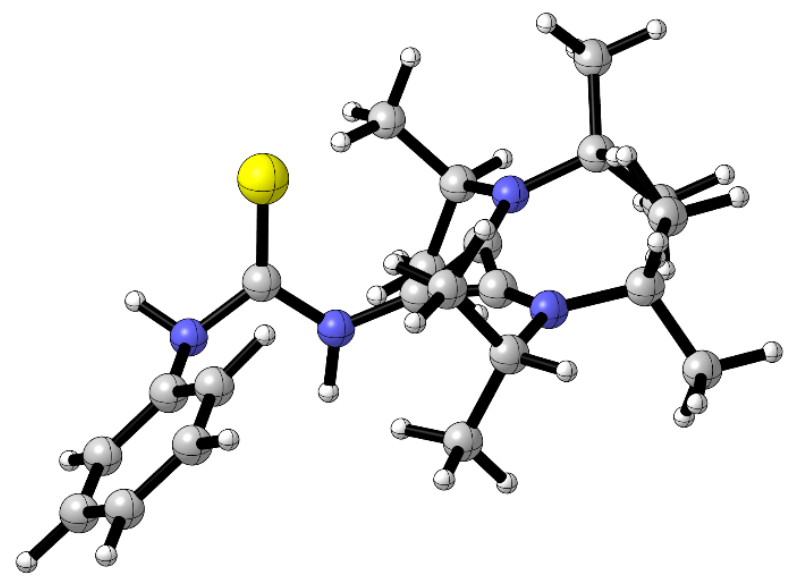

Figure S76. Optimized Structure of E,Z-Z,Z-Rotation-4

\section{- Thermochemistry -}

(0 imaginary frequencies)

Zero-point correction=

Thermal correction to Energy=

Thermal correction to Enthalpy=

Thermal correction to Gibbs Free Energy=

Sum of electronic and zero-point Energies=

Sum of electronic and thermal Energies=

Sum of electronic and thermal Enthalpies=

Sum of electronic and thermal Free Energies=
0.547304 (Hartree/Particle)

0.576924

0.577868

0.486057

$-1475.762748$

$-1475.733128$

$-1475.732184$

$-1475.823995$

$\begin{array}{crrc}\mathrm{C} & 1.61246900 & -1.59891900 & 0.35948800 \\ \mathrm{~S} & 1.08254400 & -1.74134900 & 1.90923200 \\ \mathrm{~N} & 0.89997100 & -0.95491500 & -0.62084800 \\ \mathrm{H} & 1.33883100 & -0.86549700 & -1.52794200 \\ \mathrm{~N} & 2.87222800 & -1.96090300 & -0.12273100 \\ \mathrm{C} & 3.91711500 & -0.96996700 & -0.15178800 \\ \mathrm{C} & 5.10236900 & -1.30634900 & -0.80134000 \\ \mathrm{C} & 3.77170400 & 0.28652300 & 0.42454000 \\ \mathrm{C} & 6.14183400 & -0.39055200 & -0.85762500 \\ \mathrm{H} & 5.20070800 & -2.28258000 & -1.26250300 \\ \mathrm{C} & 4.81196300 & 1.20591200 & 0.34353100 \\ \mathrm{H} & 2.86839400 & 0.54157900 & 0.96361500 \\ \mathrm{C} & 5.99838300 & 0.87278600 & -0.29314400 \\ \mathrm{H} & 6.80906400 & 1.58905200 & -0.34558000 \\ \mathrm{H} & 3.19797000 & -2.77379800 & 0.38812200 \\ \mathrm{H} & 4.69634200 & 2.18118500 & 0.80246100 \\ \mathrm{H} & 7.06502800 & -0.66128200 & -1.35596300\end{array}$

$\begin{array}{lrrr}\mathrm{C} & -0.32332700 & -0.35800400 & -0.40969400 \\ \mathrm{C} & -1.68013800 & -0.49018600 & -0.26437000 \\ \mathrm{C} & -1.04441800 & 0.79021900 & -0.22364500 \\ \mathrm{~N} & -2.76400900 & -1.20872700 & -0.20631100 \\ \mathrm{~N} & -1.10326700 & 2.08727800 & -0.09997400 \\ \mathrm{C} & -4.06585800 & -0.57329000 & 0.11603800 \\ \mathrm{C} & -2.70857800 & -2.65703700 & -0.55160100 \\ \mathrm{C} & -2.40036900 & 2.80318900 & -0.09208300 \\ \mathrm{C} & 0.16346300 & 2.84166300 & 0.09675000 \\ \mathrm{C} & -4.87401400 & -0.34378900 & -1.15654300 \\ \mathrm{H} & -3.80973300 & 0.39151000 & 0.55783200 \\ \mathrm{C} & -4.82839200 & -1.35720300 & 1.17822000 \\ \mathrm{C} & -2.17161600 & -3.48089700 & 0.61219900 \\ \mathrm{H} & -3.74730100 & -2.93409400 & -0.73919400 \\ \mathrm{C} & -1.92701500 & -2.88611300 & -1.84061900 \\ \mathrm{H} & -3.15064700 & 2.03644800 & -0.29112900 \\ \mathrm{C} & -2.67810000 & 3.42213800 & 1.27290600\end{array}$




$\begin{array}{lrrrrrrr}\mathrm{C} & -2.46823600 & 3.81338400 & -1.23370800 & \mathrm{H} & -0.85683100 & -2.71524700 & -1.69745300 \\ \mathrm{H} & -0.12799800 & 3.89235400 & 0.07838500 & \mathrm{H} & -2.05041700 & -3.92381500 & -2.15480600 \\ \mathrm{C} & 0.75516900 & 2.52231000 & 1.46627600 & \mathrm{H} & -1.95060900 & 4.20117200 & 1.51461800 \\ \mathrm{C} & 1.14278700 & 2.60381500 & -1.04764100 & \mathrm{H} & -3.66670200 & 3.88523500 & 1.27462200 \\ \mathrm{H} & -4.31823000 & 0.27154600 & -1.86891000 & \mathrm{H} & -2.64903400 & 2.66644700 & 2.06118500 \\ \mathrm{H} & -5.12311400 & -1.28984100 & -1.64410400 & \mathrm{H} & -2.26690800 & 3.33427900 & -2.19403600 \\ \mathrm{H} & -5.81143900 & 0.16394300 & -0.92090400 & \mathrm{H} & -3.46787900 & 4.25038500 & -1.27249300 \\ \mathrm{H} & -5.14768800 & -2.33836100 & 0.82022800 & \mathrm{H} & -1.75989000 & 4.63408400 & -1.09931600 \\ \mathrm{H} & -4.22719300 & -1.48991900 & 2.07911400 & \mathrm{H} & 1.69956400 & 3.05363900 & 1.60056600 \\ \mathrm{H} & -5.72829200 & -0.80163500 & 1.44873700 & \mathrm{H} & 0.07678900 & 2.82000300 & 2.26769500 \\ \mathrm{H} & -1.14153300 & -3.19964300 & 0.84417100 & \mathrm{H} & 0.94929300 & 1.45099700 & 1.57191300 \\ \mathrm{H} & -2.77224000 & -3.34352700 & 1.51214800 & \mathrm{H} & 1.56859200 & 1.59835900 & -1.01337700 \\ \mathrm{H} & -2.18681600 & -4.54103500 & 0.35055500 & \mathrm{H} & 0.66372800 & 2.76139900 & -2.01625000 \\ \mathrm{H} & -2.28886800 & -2.24146800 & -2.64504500 & \mathrm{H} & 1.97672400 & 3.30278100 & -0.96250000\end{array}$

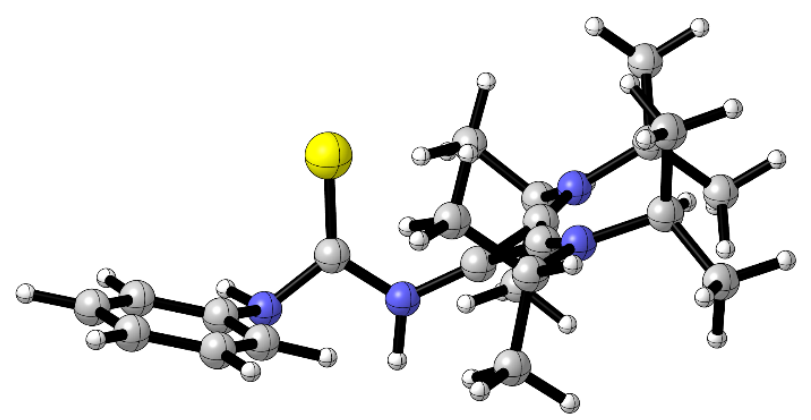

Figure S77. Optimized Structure of E,Z-Z,Z-Rotation-5

- Thermochemistry -

(0 imaginary frequencies)

Zero-point correction $=$

Thermal correction to Energy=

Thermal correction to Enthalpy=

Thermal correction to Gibbs Free Energy=

Sum of electronic and zero-point Energies=

Sum of electronic and thermal Energies=

Sum of electronic and thermal Enthalpies=

Sum of electronic and thermal Free Energies=
0.546939 (Hartree/Particle)

$$
0.576622
$$

0.577566

$$
\begin{gathered}
0.485288 \\
-1475.758072 \\
-1475.728389 \\
-1475.727444 \\
-1475.819722
\end{gathered}
$$

$\begin{array}{lrrr}\mathrm{C} & 1.65172700 & -1.45137300 & -0.07734500 \\ \mathrm{~S} & 1.28562600 & -1.70304300 & 1.49874700 \\ \mathrm{~N} & 0.81729100 & -0.82548400 & -0.96307100 \\ \mathrm{H} & 1.16700900 & -0.70303300 & -1.90447200 \\ \mathrm{~N} & 2.88846100 & -1.75223700 & -0.71081700 \\ \mathrm{C} & 3.97307600 & -0.88346400 & -0.35223200 \\ \mathrm{C} & 5.14060100 & -1.41257600 & 0.18524400 \\ \mathrm{C} & 3.87000500 & 0.48873900 & -0.56583200 \\ \mathrm{C} & 6.20118200 & -0.57251000 & 0.49983600 \\ \mathrm{H} & 5.22272000 & -2.47974500 & 0.36277700 \\ \mathrm{C} & 4.92648800 & 1.32347100 & -0.23113400 \\ \mathrm{H} & 2.97219900 & 0.90751400 & -1.00537200 \\ \mathrm{C} & 6.09748200 & 0.79757500 & 0.30060500 \\ \mathrm{H} & 6.92352900 & 1.45036200 & 0.55427600\end{array}$

$\begin{array}{lrrr}\mathrm{H} & 3.14087200 & -2.70304100 & -0.46575300 \\ \mathrm{H} & 4.83852200 & 2.39049800 & -0.40100000 \\ \mathrm{H} & 7.10852600 & -0.99519300 & 0.91444200 \\ \mathrm{C} & -0.40641700 & -0.29973600 & -0.60493400 \\ \mathrm{C} & -1.72388700 & -0.49860300 & -0.28437900 \\ \mathrm{C} & -1.13440500 & 0.80455100 & -0.25561700 \\ \mathrm{~N} & -2.76013700 & -1.26765600 & -0.11518000 \\ \mathrm{~N} & -1.21049700 & 2.08838300 & -0.04543200 \\ \mathrm{C} & -4.02604300 & -0.71164800 & 0.42436800 \\ \mathrm{C} & -2.69558300 & -2.69563200 & -0.53549700 \\ \mathrm{C} & -2.50891500 & 2.75529100 & 0.20831600 \\ \mathrm{C} & 0.04587000 & 2.88604700 & -0.05840500 \\ \mathrm{C} & -5.03504000 & -0.48862000 & -0.69689900 \\ \mathrm{H} & -3.75215700 & 0.25244500 & 0.85671300\end{array}$




$\begin{array}{lrrrrrrr}\mathrm{C} & -4.57432500 & -1.56823900 & 1.56057000 & \mathrm{H} & -2.46354800 & -3.48252000 & 1.47973200 \\ \mathrm{C} & -1.97894600 & -3.54738900 & 0.50508100 & \mathrm{H} & -1.98373000 & -4.59307100 & 0.19030700 \\ \mathrm{H} & -3.73863300 & -3.00987200 & -0.60069000 & \mathrm{H} & -2.56481800 & -2.16532000 & -2.64151400 \\ \mathrm{C} & -2.08236400 & -2.83381700 & -1.92468500 & \mathrm{H} & -1.00794800 & -2.63222300 & -1.91298100 \\ \mathrm{H} & -3.26299500 & 1.98112900 & 0.05676800 & \mathrm{H} & -2.21470100 & -3.85894300 & -2.27449500 \\ \mathrm{C} & -2.59059000 & 3.25118600 & 1.64735500 & \mathrm{H} & -1.84019100 & 4.02019600 & 1.84840700 \\ \mathrm{C} & -2.76795800 & 3.85105000 & -0.82173100 & \mathrm{H} & -3.57144100 & 3.69362000 & 1.83197300 \\ \mathrm{H} & -0.26266900 & 3.90187800 & 0.19084700 & \mathrm{H} & -2.44104700 & 2.43291200 & 2.35541800 \\ \mathrm{C} & 1.00706900 & 2.40245700 & 1.02141200 & \mathrm{H} & -2.70510900 & 3.45692200 & -1.83816300 \\ \mathrm{C} & 0.66178800 & 2.90293100 & -1.45318100 & \mathrm{H} & -3.77017100 & 4.25741400 & -0.67353900 \\ \mathrm{H} & -4.63009800 & 0.17402200 & -1.46609900 & \mathrm{H} & -2.06349100 & 4.68034800 & -0.72512300 \\ \mathrm{H} & -5.31821600 & -1.43149500 & -1.17161700 & \mathrm{H} & 1.88563000 & 3.05001700 & 1.04899800 \\ \mathrm{H} & -5.94368300 & -0.03482700 & -0.29620300 & \mathrm{H} & 0.53275900 & 2.42608800 & 2.00431400 \\ \mathrm{H} & -4.89575000 & -2.55393400 & 1.21684600 & \mathrm{H} & 1.35771800 & 1.38474500 & 0.83489800 \\ \mathrm{H} & -3.83244200 & -1.69611200 & 2.35070700 & \mathrm{H} & 0.97955200 & 1.90210300 & -1.75836000 \\ \mathrm{H} & -5.44711900 & -1.07229200 & 1.98921800 & \mathrm{H} & -0.04450800 & 3.28230000 & -2.19395900 \\ \mathrm{H} & -0.94069900 & -3.22734100 & 0.61972500 & \mathrm{H} & 1.54539700 & 3.54392500 & -1.46096300\end{array}$

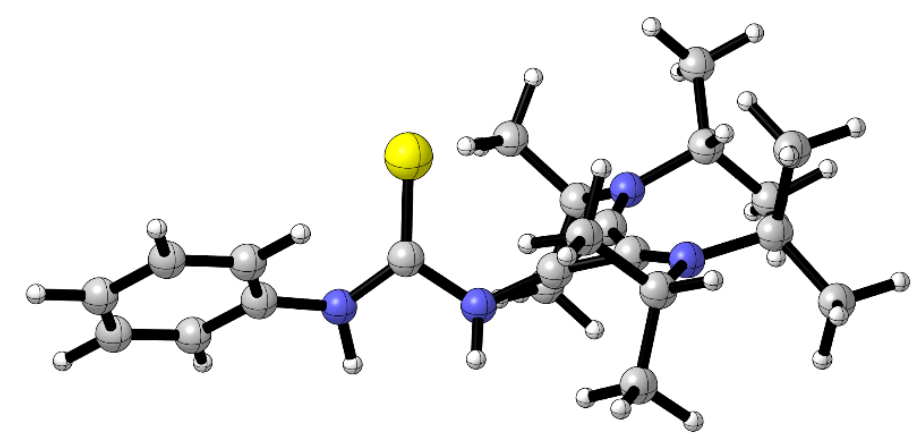

Figure S78. Optimized Structure of E,Z-Z,Z-Rotation-6

- Thermochemistry -

(0 imaginary frequencies)

Zero-point correction $=$

Thermal correction to Energy=

0.547186 (Hartree/Particle)

Thermal correction to Enthalpy=

0.577743

Thermal correction to Gibbs Free Energy $=0.483791$

Sum of electronic and zero-point Energies $=$

$-1475.765377$

Sum of electronic and thermal Energies $=$

$-1475.734820$

Sum of electronic and thermal Enthalpies $=\quad-1475.733876$

Sum of electronic and thermal Free Energies $=\quad-1475.828773$

$\begin{array}{ccrrrrrc}\mathrm{C} & 1.89312000 & -0.26152200 & -0.07761800 & \mathrm{C} & 6.73658000 & -0.30768500 & -0.56919700 \\ \mathrm{~S} & 1.58869800 & -0.77814700 & 1.45609900 & \mathrm{H} & 5.28044500 & -1.41973500 & -1.69630900 \\ \mathrm{~N} & 0.87181900 & 0.24044400 & -0.90207000 & \mathrm{C} & 5.85165200 & 1.23116400 & 1.05031100 \\ \mathrm{H} & 1.11613200 & 0.99700400 & -1.52669200 & \mathrm{H} & 3.71163400 & 1.36169100 & 1.16720900 \\ \mathrm{~N} & 3.05328300 & -0.41696300 & -0.76898100 & \mathrm{C} & 6.94255900 & 0.63714600 & 0.42821300 \\ \mathrm{C} & 4.36045700 & -0.05631400 & -0.31622200 & \mathrm{H} & 7.94976700 & 0.90708600 & 0.72122400 \\ \mathrm{C} & 5.44684900 & -0.66381700 & -0.93656000 & \mathrm{H} & 2.95341400 & -0.38296900 & -1.77481700 \\ \mathrm{C} & 4.55744500 & 0.89417300 & 0.67867900 & \mathrm{H} & 6.00534400 & 1.96871800 & 1.82895000\end{array}$




$\begin{array}{lrrrrrrr}\mathrm{H} & 7.58153200 & -0.78052600 & -1.05499800 & \mathrm{H} & -4.35921700 & -2.97778000 & -1.17741800 \\ \mathrm{C} & -0.44430100 & 0.13798300 & -0.54931400 & \mathrm{H} & -5.49308700 & -1.95994100 & -0.29046700 \\ \mathrm{C} & -1.52104100 & -0.65593200 & -0.24470900 & \mathrm{H} & -3.52884600 & -3.93895200 & 1.14603500 \\ \mathrm{C} & -1.63124200 & 0.75725000 & -0.26736700 & \mathrm{H} & -2.93647700 & -2.78646200 & 2.36008100 \\ \mathrm{~N} & -2.08343000 & -1.81917900 & -0.04694900 & \mathrm{H} & -4.65650700 & -2.85577300 & 1.95620000 \\ \mathrm{~N} & -2.32473400 & 1.85906000 & -0.12500500 & \mathrm{H} & 0.33030200 & -3.01877300 & 0.74157900 \\ \mathrm{C} & -3.47418300 & -1.86334300 & 0.46963300 & \mathrm{H} & -1.03835700 & -3.86845700 & 1.47160100 \\ \mathrm{C} & -1.42983600 & -3.07202600 & -0.51861700 & \mathrm{H} & -0.12072400 & -4.63207000 & 0.16890400 \\ \mathrm{C} & -3.79004500 & 1.81514800 & 0.07842300 & \mathrm{H} & -1.36457700 & -2.37728200 & -2.58123500 \\ \mathrm{C} & -1.60170200 & 3.15417200 & -0.09120000 & \mathrm{H} & 0.18836600 & -2.24381500 & -1.72052500 \\ \mathrm{C} & -4.47236600 & -2.01388400 & -0.67502900 & \mathrm{H} & -0.39580200 & -3.81765300 & -2.24557300 \\ \mathrm{H} & -3.62676000 & -0.89635400 & 0.95367500 & \mathrm{H} & -3.88485000 & 3.18103800 & 1.77100000 \\ \mathrm{C} & -3.64787000 & -2.92922000 & 1.54522700 & \mathrm{H} & -5.22080500 & 2.03523800 & 1.67924200 \\ \mathrm{C} & -0.51258900 & -3.68056000 & 0.53557500 & \mathrm{H} & -3.62436200 & 1.48680100 & 2.21886400 \\ \mathrm{H} & -2.26010300 & -3.75741200 & -0.70301000 & \mathrm{H} & -4.24227500 & 2.42969000 & -1.95709000 \\ \mathrm{C} & -0.71085800 & -2.85248600 & -1.84574400 & \mathrm{H} & -5.59430300 & 2.55334600 & -0.82344800 \\ \mathrm{H} & -4.07444900 & 0.78187800 & -0.12596200 & \mathrm{H} & -4.31235500 & 3.75629100 & -0.78019100 \\ \mathrm{C} & -4.14608600 & 2.14869100 & 1.52346200 & \mathrm{H} & -0.06751700 & 4.14130300 & 1.05619100 \\ \mathrm{C} & -4.51747600 & 2.69422300 & -0.93403800 & \mathrm{H} & -0.94716600 & 2.90105700 & 1.96176300 \\ \mathrm{H} & -2.35395800 & 3.89306200 & 0.18772700 & \mathrm{H} & 0.27132400 & 2.43276600 & 0.76902300 \\ \mathrm{C} & -0.52324900 & 3.15174500 & 0.98757200 & \mathrm{H} & -0.31598400 & 2.79403800 & -1.80827900 \\ \mathrm{C} & -1.06694800 & 3.51602300 & -1.47293100 & \mathrm{H} & -1.86727100 & 3.54203200 & -2.21418100 \\ \mathrm{H} & -4.34410900 & -1.22491000 & -1.42128800 & \mathrm{H} & -0.58934400 & 4.49707600 & -1.44562600 \\ & & & & & & \end{array}$

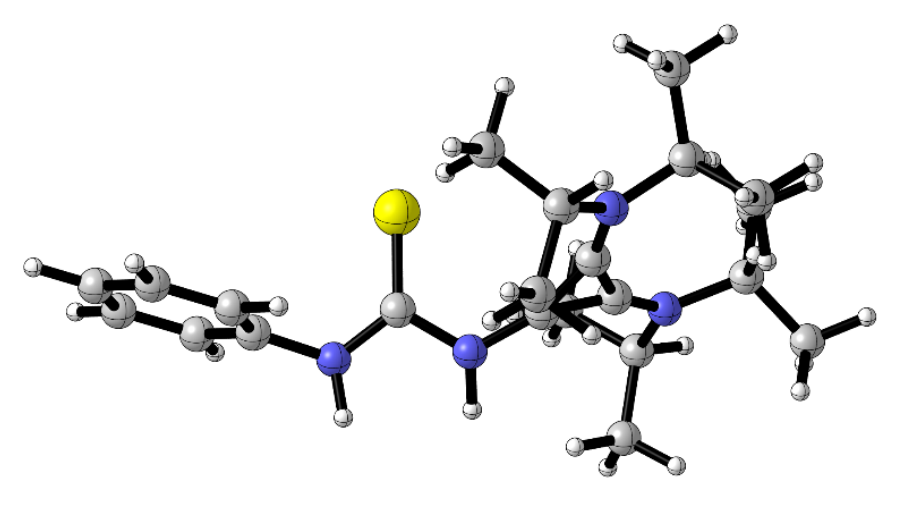

Figure S79. Optimized Structure of E,Z-Z,Z-Rotation-7

- Thermochemistry -

(0 imaginary frequencies)

Zero-point correction $=$

Thermal correction to Energy=

Thermal correction to Enthalpy=

0.546433 (Hartree/Particle)

Thermal correction to Gibbs Free Energy=

0.576441

0.577385

Sum of electronic and zero-point Energies=

0.483912

Sum of electronic and thermal Energies=

$-1475.768761$

Sum of electronic and thermal Enthalpies=

$-1475.738752$

$-1475.737808$

Sum of electronic and thermal Free Energies $=\quad-1475.831282$

$\begin{array}{llll}\mathrm{C} & -1.83672700 & -0.62168400 & 0.14839300 \\ \mathrm{~S} & -1.68455900 & -0.06946000 & -1.40070500 \\ \mathrm{~N} & -0.72527300 & -0.76021200 & 0.99208500\end{array}$

$\begin{array}{llll}\mathrm{H} & -0.74639700 & -1.49086900 & 1.69063000 \\ \mathrm{~N} & -2.96553300 & -1.13069400 & 0.69476400 \\ \mathrm{C} & -4.27771900 & -0.67893600 & 0.30576400\end{array}$ 


\begin{tabular}{|c|c|c|c|c|c|c|c|}
\hline $\mathrm{C}$ & -5.15416100 & -1.57702000 & -0.28266500 & $\mathrm{C}$ & 3.87971300 & 2.79420200 & 0.63008400 \\
\hline $\mathrm{C}$ & -4.65921600 & 0.63278500 & 0.55973100 & $\mathrm{H}$ & 1.28778600 & 3.89384100 & 0.72317800 \\
\hline $\mathrm{C}$ & -6.43544000 & -1.15748800 & -0.61963000 & $\mathrm{C}$ & -0.43846600 & 3.30673700 & -0.40603300 \\
\hline $\mathrm{H}$ & -4.82757200 & -2.59089400 & -0.47975200 & $\mathrm{C}$ & 0.11703800 & 2.62701200 & 1.96867000 \\
\hline $\mathrm{C}$ & -5.93791000 & 1.04648600 & 0.21713100 & $\mathrm{H}$ & 4.80554900 & -1.66072100 & 1.81483300 \\
\hline $\mathrm{H}$ & -3.95613000 & 1.32352700 & 1.01227900 & $\mathrm{H}$ & 5.16542400 & -2.90068000 & 0.59686700 \\
\hline $\mathrm{C}$ & -6.82605100 & 0.15164600 & -0.37010800 & $\mathrm{H}$ & 6.10514600 & -1.41477900 & 0.64135400 \\
\hline $\mathrm{H}$ & -7.82478600 & 0.47796600 & -0.63468400 & $\mathrm{H}$ & 4.48060900 & -2.39635200 & -1.92567200 \\
\hline $\mathrm{H}$ & -2.91088900 & -1.38855800 & 1.67198000 & $\mathrm{H}$ & 3.83409000 & -0.79242900 & -2.30895000 \\
\hline $\mathrm{H}$ & -6.24214600 & 2.06842700 & 0.40880900 & $\mathrm{H}$ & 5.53061000 & -0.98312100 & -1.83305000 \\
\hline $\mathrm{H}$ & -7.12577900 & -1.85344600 & -1.08054500 & $\mathrm{H}$ & 0.33969300 & -2.74991200 & -0.77578900 \\
\hline $\mathrm{C}$ & 0.51658300 & -0.33988600 & 0.59624800 & $\mathrm{H}$ & 1.59976000 & -2.83567700 & -2.01296500 \\
\hline $\mathrm{C}$ & 1.80540300 & -0.65037200 & 0.25810800 & $\mathrm{H}$ & 1.11051400 & -4.29517200 & -1.13987300 \\
\hline $\mathrm{C}$ & 1.35530800 & 0.69725800 & 0.27770000 & $\mathrm{H}$ & 2.76665900 & -3.31016300 & 2.12403500 \\
\hline $\mathrm{N}$ & 2.74288000 & -1.54158400 & 0.06248200 & $\mathrm{H}$ & 1.06368300 & -2.99014800 & 1.75466800 \\
\hline $\mathrm{N}$ & 1.60902000 & 1.96472400 & 0.09405500 & $\mathrm{H}$ & 1.76819900 & -4.55572100 & 1.35727700 \\
\hline $\mathrm{C}$ & 4.14350000 & -1.13254900 & -0.18647200 & $\mathrm{H}$ & 2.38819000 & 4.37507400 & -1.15787600 \\
\hline $\mathrm{C}$ & 2.36389700 & -2.97608900 & 0.01327400 & $\mathrm{H}$ & 3.73130900 & 3.62295500 & -2.01280200 \\
\hline $\mathrm{C}$ & 2.91773500 & 2.36941900 & -0.47565400 & $\mathrm{H}$ & 2.07721600 & 3.09009300 & -2.34286500 \\
\hline $\mathrm{C}$ & 0.66799500 & 3.00358500 & 0.59728500 & $\mathrm{H}$ & 3.99690400 & 2.00552200 & 1.37830900 \\
\hline $\mathrm{C}$ & 5.10257200 & -1.82492300 & 0.77700600 & $\mathrm{H}$ & 4.86171200 & 3.01484900 & 0.20647800 \\
\hline $\mathrm{H}$ & 4.17403300 & -0.06538700 & 0.03981400 & $\mathrm{H}$ & 3.52890700 & 3.69589700 & 1.13831700 \\
\hline $\mathrm{C}$ & 4.51442000 & -1.33870100 & -1.65117500 & $\mathrm{H}$ & -1.07195800 & 4.10819300 & -0.01891200 \\
\hline $\mathrm{C}$ & 1.28938700 & -3.22289600 & -1.04067400 & $\mathrm{H}$ & -0.03451800 & 3.62812000 & -1.36634700 \\
\hline $\mathrm{H}$ & 3.26994300 & -3.49715800 & -0.29837500 & $\mathrm{H}$ & -1.05746000 & 2.42395200 & -0.57581300 \\
\hline $\mathrm{C}$ & 1.97201900 & -3.48408200 & 1.39652600 & $\mathrm{H}$ & -0.58336200 & 1.78982500 & 1.90639800 \\
\hline $\mathrm{H}$ & 3.30544500 & 1.47198000 & -0.96181700 & $\mathrm{H}$ & 0.91868400 & 2.36813200 & 2.66465600 \\
\hline $\mathrm{C}$ & 2.75732000 & 3.42886200 & -1.55975200 & $\mathrm{H}$ & -0.42698500 & 3.47836200 & 2.38124700 \\
\hline
\end{tabular}

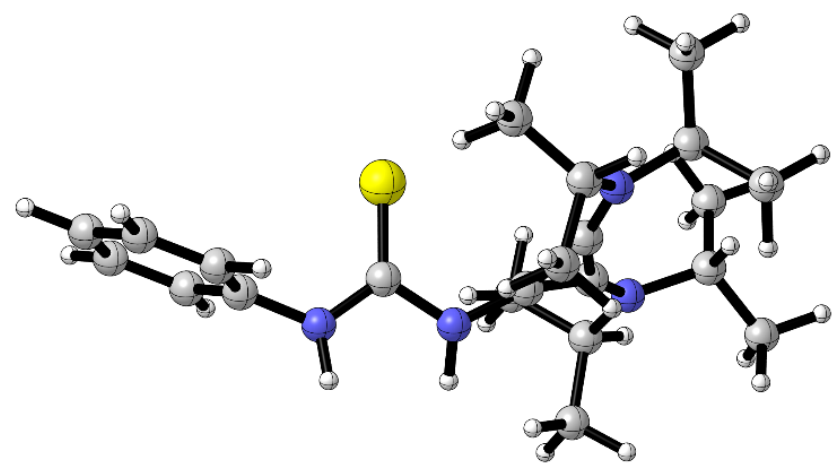

Figure S80. Optimized Structure of E,Z-Z,Z-Rotation-8

- Thermochemistry -

(0 imaginary frequencies)

Zero-point correction $=$

Thermal correction to Energy=

0.546839 (Hartree/Particle)

Thermal correction to Enthalpy=

0.577604

Thermal correction to Gibbs Free Energy $=\quad 0.483102$

Sum of electronic and zero-point Energies $=\quad-1475.772273$

Sum of electronic and thermal Energies $=\quad-1475.741508$

Sum of electronic and thermal Enthalpies $=\quad-1475.740564$

Sum of electronic and thermal Free Energies $=\quad-1475.836010$ 


$\begin{array}{lrrr}\mathrm{C} & 1.85954400 & -0.43257000 & -0.27607400 \\ \mathrm{~S} & 1.74136500 & 0.09667500 & 1.28235800 \\ \mathrm{~N} & 0.72273000 & -0.66125600 & -1.07549600 \\ \mathrm{H} & 0.75497000 & -1.46349300 & -1.69038100 \\ \mathrm{~N} & 2.99237500 & -0.79471100 & -0.91019000 \\ \mathrm{C} & 4.30463400 & -0.53240700 & -0.38646000 \\ \mathrm{C} & 4.96225400 & -1.53016400 & 0.31740600 \\ \mathrm{C} & 4.90250600 & 0.70029500 & -0.61125500 \\ \mathrm{C} & 6.23844500 & -1.28868400 & 0.80776600 \\ \mathrm{H} & 4.47049300 & -2.48107100 & 0.48345700 \\ \mathrm{C} & 6.18031400 & 0.93382600 & -0.12198200 \\ \mathrm{H} & 4.36637700 & 1.47058500 & -1.15432200 \\ \mathrm{C} & 6.84650300 & -0.05898000 & 0.58730300 \\ \mathrm{H} & 7.84240400 & 0.12737300 & 0.97112400 \\ \mathrm{H} & 2.94076900 & -0.92448600 & -1.91139000 \\ \mathrm{H} & 6.65449100 & 1.89302200 & -0.29102200 \\ \mathrm{H} & 6.75770300 & -2.06147300 & 1.36148200 \\ \mathrm{C} & -0.52044500 & -0.29787100 & -0.63827300 \\ \mathrm{C} & -1.78460200 & -0.66731100 & -0.26700300 \\ \mathrm{C} & -1.39562400 & 0.69608100 & -0.28230900 \\ \mathrm{~N} & -2.67501600 & -1.60402400 & -0.05567700 \\ \mathrm{~N} & -1.70134600 & 1.94910000 & -0.07220900 \\ \mathrm{C} & -4.08487100 & -1.26398900 & 0.23948600 \\ \mathrm{C} & -2.22823300 & -3.01875800 & -0.02932900 \\ \mathrm{C} & -3.01343900 & 2.27813700 & 0.53715700 \\ \mathrm{C} & -0.83758000 & 3.03985500 & -0.60164600 \\ \mathrm{C} & -5.03911100 & -1.99996600 & -0.69614400 \\ \mathrm{H} & -4.17516900 & -0.19894200 & 0.01999500 \\ \mathrm{C} & -4.39855600 & -1.49376100 & 1.71416200 \\ \mathrm{C} & -1.11127700 & -3.22332200 & 0.98915100 \\ \mathrm{H} & -3.09789100 & -3.58433000 & 0.30748200\end{array}$

$\begin{array}{lrrr}\mathrm{C} & -1.85908500 & -3.50039700 & -1.42822900 \\ \mathrm{H} & -3.33174200 & 1.36096800 & 1.03702200 \\ \mathrm{C} & -2.88476700 & 3.34878400 & 1.61434000 \\ \mathrm{C} & -4.03301300 & 2.64028500 & -0.53894800 \\ \mathrm{H} & -1.51529400 & 3.88833600 & -0.71865900 \\ \mathrm{C} & 0.27069200 & 3.42254200 & 0.37232400 \\ \mathrm{C} & -0.29453900 & 2.69034600 & -1.98331500 \\ \mathrm{H} & -4.78683300 & -1.81429800 & -1.74218500 \\ \mathrm{H} & -5.03801500 & -3.07863800 & -0.52229300 \\ \mathrm{H} & -6.05701000 & -1.64528100 & -0.52355900 \\ \mathrm{H} & -4.29335300 & -2.54802000 & 1.98374100 \\ \mathrm{H} & -3.73191300 & -0.90962300 & 2.35323900 \\ \mathrm{H} & -5.42816700 & -1.20019200 & 1.92831400 \\ \mathrm{H} & -0.19254000 & -2.70563300 & 0.69959600 \\ \mathrm{H} & -1.40845100 & -2.85658900 & 1.97344900 \\ \mathrm{H} & -0.88146900 & -4.28707700 & 1.07450200 \\ \mathrm{H} & -2.68560400 & -3.36201600 & -2.12719200 \\ \mathrm{H} & -0.98989400 & -2.96029200 & -1.81597200 \\ \mathrm{H} & -1.60167900 & -4.56094200 & -1.40375000 \\ \mathrm{H} & -2.57789100 & 4.31230300 & 1.20145100 \\ \mathrm{H} & -3.85699100 & 3.49160000 & 2.08980900 \\ \mathrm{H} & -2.16936300 & 3.05022800 & 2.38207900 \\ \mathrm{H} & -4.12273200 & 1.84476100 & -1.28371400 \\ \mathrm{H} & -5.01399600 & 2.80025800 & -0.08653400 \\ \mathrm{H} & -3.75491800 & 3.56114800 & -1.05794200 \\ \mathrm{H} & 0.82244000 & 4.27763200 & -0.02493000 \\ \mathrm{H} & -0.12805000 & 3.70028800 & 1.34812700 \\ \mathrm{H} & 0.96707900 & 2.59377700 & 0.51182300 \\ \mathrm{H} & 0.45087800 & 1.89219900 & -1.93390200 \\ \mathrm{H} & -1.09467300 & 2.38669600 & -2.66279700 \\ \mathrm{H} & 0.19448600 & 3.56881800 & -2.40763600\end{array}$

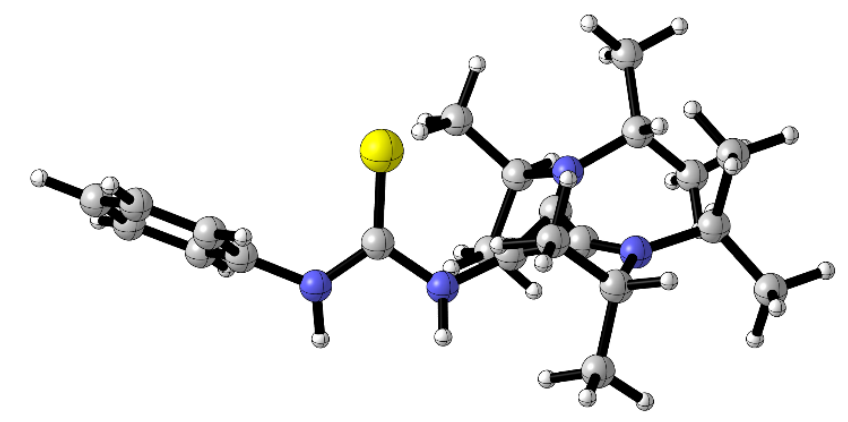

Figure S81. Optimized Structure of E,Z-Z,Z-Rotation-9

- Thermochemistry -

(0 imaginary frequencies)

Zero-point correction $=$

Thermal correction to Energy=

Thermal correction to Enthalpy=

Thermal correction to Gibbs Free Energy=

Sum of electronic and zero-point Energies=

\author{
0.546621 (Hartree/Particle) \\ 0.577436 \\ 0.578380 \\ 0.482853 \\ $-1475.772996$
}


Sum of electronic and thermal Energies= Sum of electronic and thermal Enthalpies= Sum of electronic and thermal Free Energies=
$-1475.742181$

$-1475.741237$

$-1475.836765$

$\begin{array}{lrrr}\mathrm{C} & -1.86129300 & -0.41135300 & -0.27727200 \\ \mathrm{~S} & -1.74169800 & 0.12570000 & 1.27796200 \\ \mathrm{~N} & -0.72351200 & -0.66044300 & -1.07079300 \\ \mathrm{H} & -0.76007300 & -1.47398500 & -1.67029600 \\ \mathrm{~N} & -2.99511000 & -0.74458200 & -0.92549800 \\ \mathrm{C} & -4.30900700 & -0.52305700 & -0.39310500 \\ \mathrm{C} & -5.02962000 & 0.59433300 & -0.79129400 \\ \mathrm{C} & -4.84727400 & -1.44251700 & 0.49588500 \\ \mathrm{C} & -6.31021500 & 0.79103000 & -0.29092000 \\ \mathrm{H} & -4.58897600 & 1.30718600 & -1.47948300 \\ \mathrm{C} & -6.12269200 & -1.23383300 & 1.00057600 \\ \mathrm{H} & -4.26391000 & -2.30544700 & 0.79345400 \\ \mathrm{C} & -6.85426600 & -0.11947600 & 0.60659200 \\ \mathrm{H} & -7.85071600 & 0.03977800 & 1.00092800 \\ \mathrm{H} & -2.93976200 & -0.85092200 & -1.92890400 \\ \mathrm{H} & -6.54631800 & -1.94389400 & 1.70040800 \\ \mathrm{H} & -6.87929000 & 1.66027800 & -0.59770100 \\ \mathrm{C} & 0.51988800 & -0.29590900 & -0.63753600 \\ \mathrm{C} & 1.39445000 & 0.69714800 & -0.27697800 \\ \mathrm{C} & 1.78578100 & -0.66506300 & -0.27205100 \\ \mathrm{~N} & 1.69986500 & 1.94899900 & -0.05744600 \\ \mathrm{~N} & 2.67734500 & -1.60300600 & -0.06895300 \\ \mathrm{C} & 3.01394900 & 2.27098200 & 0.55179100 \\ \mathrm{C} & 0.84108000 & 3.04589000 & -0.58263500 \\ \mathrm{C} & 4.08706900 & -1.26444800 & 0.22813000 \\ \mathrm{C} & 2.23149400 & -3.01798100 & -0.05429200 \\ \mathrm{C} & 4.03455000 & 2.63197500 & -0.52384900 \\ \mathrm{H} & 3.32869600 & 1.35111200 & 1.04894100 \\ \mathrm{C} & 2.89139600 & 3.33920300 & 1.63202400 \\ \mathrm{C} & -0.25862900 & 3.44096300 & 0.39616600 \\ \mathrm{H} & 1.52544400 & 3.88839600 & -0.70486700\end{array}$

$\begin{array}{lrrr}\mathrm{C} & 0.28671400 & 2.70003200 & -1.96066100 \\ \mathrm{H} & 4.17665500 & -0.19775700 & 0.01691700 \\ \mathrm{C} & 4.40135600 & -1.50504700 & 1.70100200 \\ \mathrm{C} & 5.04180100 & -1.99254200 & -0.71311100 \\ \mathrm{H} & 3.10017200 & -3.58576100 & 0.28140600 \\ \mathrm{C} & 1.11138500 & -3.23085500 & 0.95904300 \\ \mathrm{C} & 1.86742600 & -3.48935400 & -1.45810000 \\ \mathrm{H} & 4.11966700 & 1.83909900 & -1.27197400 \\ \mathrm{H} & 3.76096500 & 3.55632400 & -1.03898700 \\ \mathrm{H} & 5.01669200 & 2.78516800 & -0.07155700 \\ \mathrm{H} & 2.58918400 & 4.30540300 & 1.22198900 \\ \mathrm{H} & 2.17516700 & 3.04217400 & 2.39956300 \\ \mathrm{H} & 3.86474000 & 3.47575600 & 2.10707800 \\ \mathrm{H} & -0.96719500 & 2.62249400 & 0.53425600 \\ \mathrm{H} & 0.14628800 & 3.71000300 & 1.37175500 \\ \mathrm{H} & -0.79886500 & 4.30519600 & 0.00287600 \\ \mathrm{H} & 1.07988600 & 2.38953400 & -2.64521100 \\ \mathrm{H} & -0.46525100 & 1.90844500 & -1.90624400 \\ \mathrm{H} & -0.19784500 & 3.58234300 & -2.38208800 \\ \mathrm{H} & 4.29713400 & -2.56137700 & 1.96277200 \\ \mathrm{H} & 5.43076800 & -1.21223900 & 1.91716000 \\ \mathrm{H} & 3.73438100 & -0.92620900 & 2.34455100 \\ \mathrm{H} & 4.78910200 & -1.79954600 & -1.75772200 \\ \mathrm{H} & 6.05938800 & -1.63809100 & -0.53818400 \\ \mathrm{H} & 5.04190500 & -3.07246000 & -0.54709500 \\ \mathrm{H} & 0.88106600 & -4.29522600 & 1.03483700 \\ \mathrm{H} & 1.40546100 & -2.87229800 & 1.94727600 \\ \mathrm{H} & 0.19371000 & -2.71061100 & 0.67070500 \\ \mathrm{H} & 1.00185300 & -2.94411300 & -1.84688700 \\ \mathrm{H} & 2.69752100 & -3.34803500 & -2.15218500 \\ \mathrm{H} & 1.60736300 & -4.54942400 & -1.44248100\end{array}$


10. Conformational Search for $\alpha$ - vs. $\beta$-Stereofacial Alcohol Addition

Computational Methods and Details:

Force Field $=$ OPLS3

Dielectric constant $=1.0$

Method $=$ PRCG

Convergence threshold $=0.05$

Maximum interactions 2500

Mixed torsional/Low-mode sampling

Maximum number of steps $=100$

100 steps per rotatable bond

Number of structures to save for each search $=100$

Energy window for saving structures $21.0 \mathrm{~kJ} / \mathrm{mol}$

Maximum atom deviation Cutoff $=0.5$ angstrom

Probability of a torsion rotation $/$ molecule translation $=0.5$

Minimum distance for low-mode move $=3.0$

Maximum distance for a low-mode move $=6.0$

From these searches

( $\beta$ ) Bottom face 49 conformers

$(\alpha)$ Top face 27 conformers 


\section{Experimental Procedures}

\section{NMR Binding Studies}

Scheme S4. Interaction involving thiourea $1 \cdot \mathrm{BF}_{4}^{-}$with DHP.
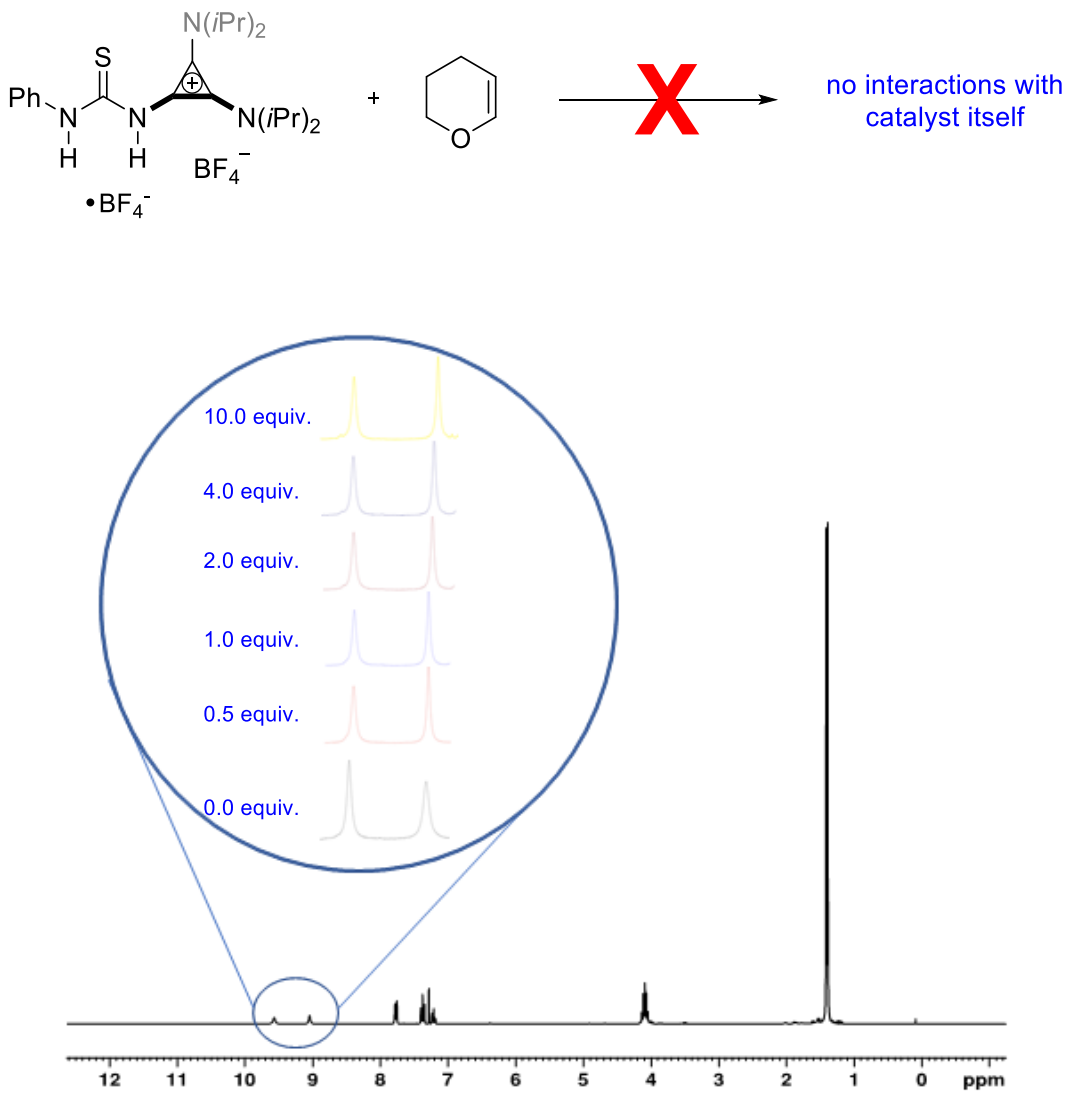

Figure S82. ${ }^{1} \mathrm{H}$ NMR spectra at $300 \mathrm{MHz}$ in $0.5 \mathrm{~mL} \mathrm{CDCl}_{3}$ at room temperature using thiourea $\mathbf{1} \cdot \mathrm{BF}_{4}^{-}(10 \mathrm{mg}, 0.02 \mathrm{mmol})$ with titrations using DHP. 


\section{Probing the Stereochemistry of Alcohol Addition}

It was found that the majority of the products were deuterated in position 3 , while any minor byproducts (e.g., protonated products - S3c) arose from the non-deuterated catalyst (Scheme S5). Key to these findings is the kinetically controlled preference for syn product formation over a duration of 48 hours (syn: anti ratio of 70:30) that is consistent with the computed Brønsted acid mode of catalysis.

Scheme S5. Investigating the stereochemistry of addition using methanol- $\mathrm{d}_{4}$ and 4-(tert-butyl)3,4-dihydro-2H-pyran.

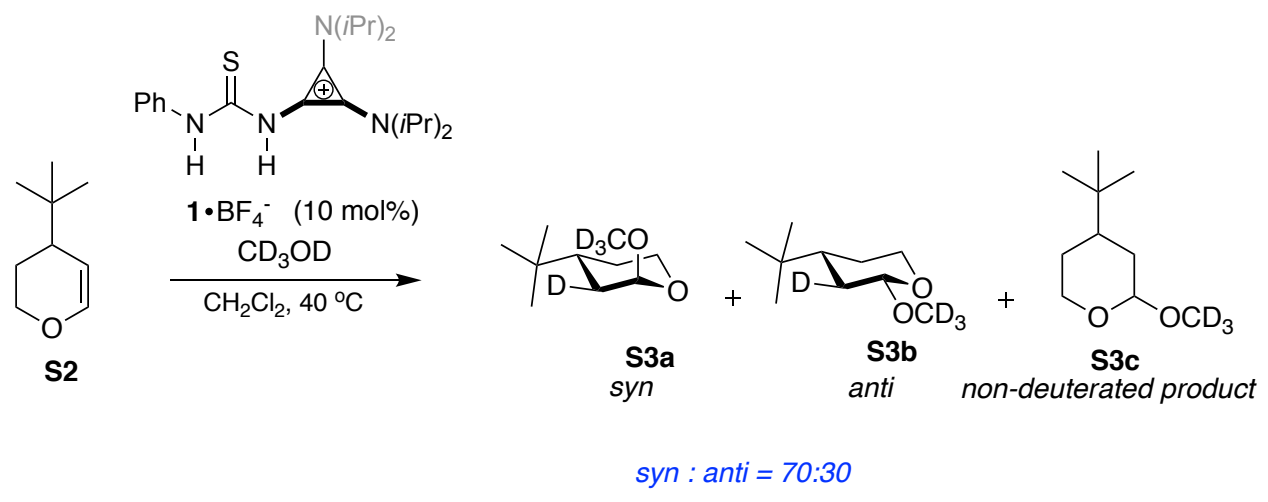

\section{Experiments Investigating Alkoxide Exchange}

To test for the potential of alkoxide exchange we performed some key experiments. First, following a literature procedure ${ }^{14}$ intermediate $\mathbf{S} \mathbf{4}$ was prepared. Next, to examine the potential for alkoxide exchange intermediate $\mathbf{S 4}$ was exposed to ethanol, which was allowed to stir for 72 hours. Over the course of the reaction, a distinguishable triplet slowly evolved indicating formation of 2ethoxytetrahydropyran (S5), and thus showing that alkoxide exchange had taken place as seen by a 3 : 1 / 2-methoxytetrahydropyran:2-ethoxytetrahydropyran ratio (Scheme S6 - path A).

Inspired by the interconversion of alkoxide adducts, vide infra, we then proceeded to use benzyl alcohol (S6) as the substrate of interest anticipating that the $\pi$-system of the benzene ring would impart additional noncovalent interactions upon contact with thiourea $\mathbf{1} \cdot \mathrm{BF}_{4}{ }^{-}$. The impetus for this being the proximal cyclopropenyl cation appended to the thiourea, which would presumably participate in catalyst-substrate $\pi$-stacking ${ }^{15}$ or cation $-\pi^{16}$ interactions. To test this theory, the same reaction parameters were implemented with the exception of using benzyl alcohol (S6) as substrate $(120 \mathrm{mg}, 1.1 \mathrm{mmol})$ as opposed to ethanol, which resulted in nearly full interconversion yielding the benzyl adduct (S7) after 72 hours (Scheme S6 - path B). To examine this exchange process we performed a computational analysis, wherein we compared the stability of the pyranylated products having varying alkoxide adducts. Our calculations demonstrated that both the ethyl and benzyl alkoxide products (S5) and (S7), respectively were lower in energy than the methyl adduct (S4). These energetic trends are consistent with the observed experimental product 
distribution. Taken together, these results suggest pyranyl product formation is reversible in the presence of thiourea $\mathbf{1} \cdot \mathrm{BF}_{4}{ }^{-}$.

Scheme S6. Probing the possibility for alkoxide exchange.

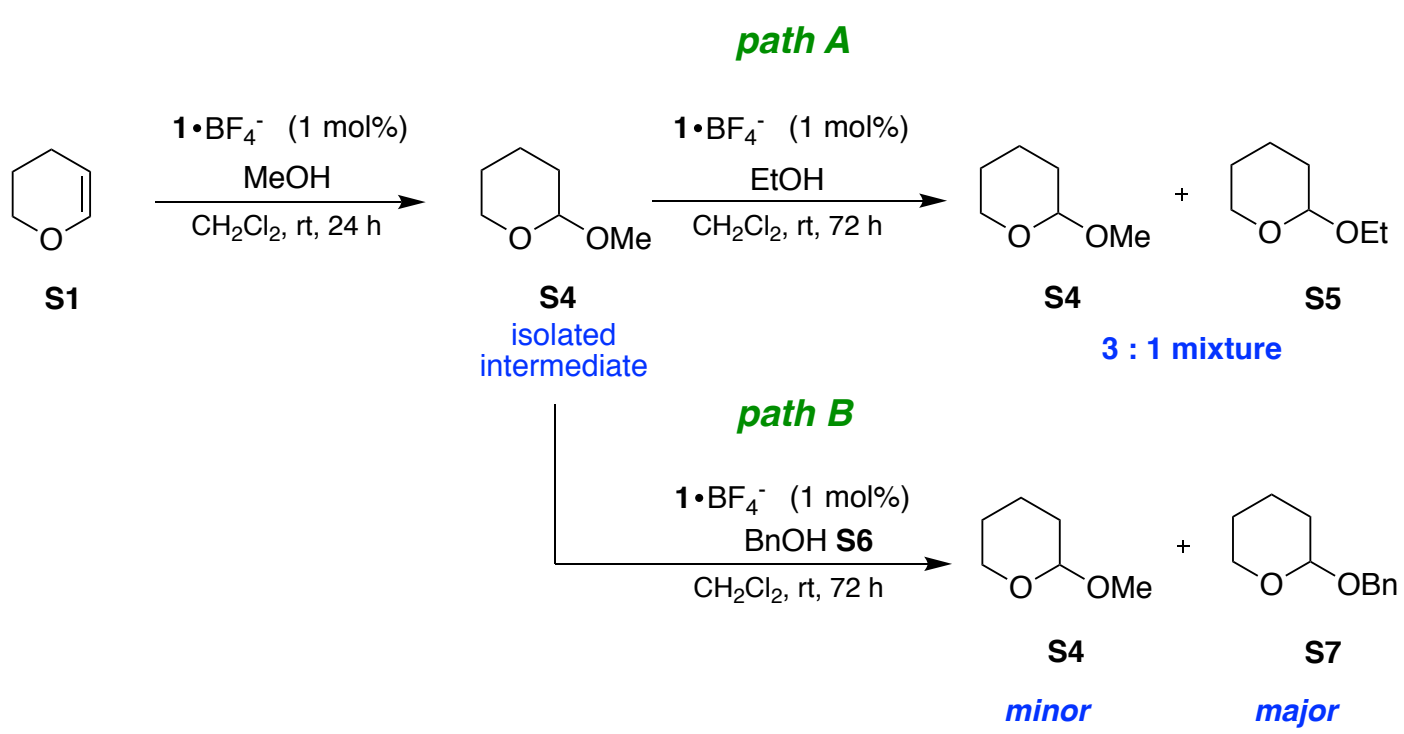

\section{Experiments Investigating Epimerization}

Finally, intrigued by the ability of thiourea catalyst $\mathbf{1} \cdot \mathrm{BF}_{4}^{-}$to induce alkoxide exchange, we applied our reaction conditions to phenyl $\left(O, 1-{ }^{2} \mathrm{H}_{2}\right)$ methanol $(\mathbf{S 8})$ to distinguish between alkoxide exchange and epimerization (Scheme S7). Based on these experimental results, there is an obvious kinetic preference for syn product formation as observed from the syn : anti ratio of 70:30 upon completion of alkoxide interconversion (Scheme S7). From this, we rule out epimerization and instead we ascribe the retention of stereochemistry to analogous mechanisms of alcohol addition. Furthermore, the stereochemical outcome is governed by a catalyst-controlled stereoselective reaction scenario. This is in accordance with kinetic control.

Scheme S7. Investigating the stereochemistry of addition using phenyl $\left(O, 1-{ }^{2} \mathrm{H}_{2}\right)$ methanol and a mixture of syn and anti product isomers (methyl adduct).
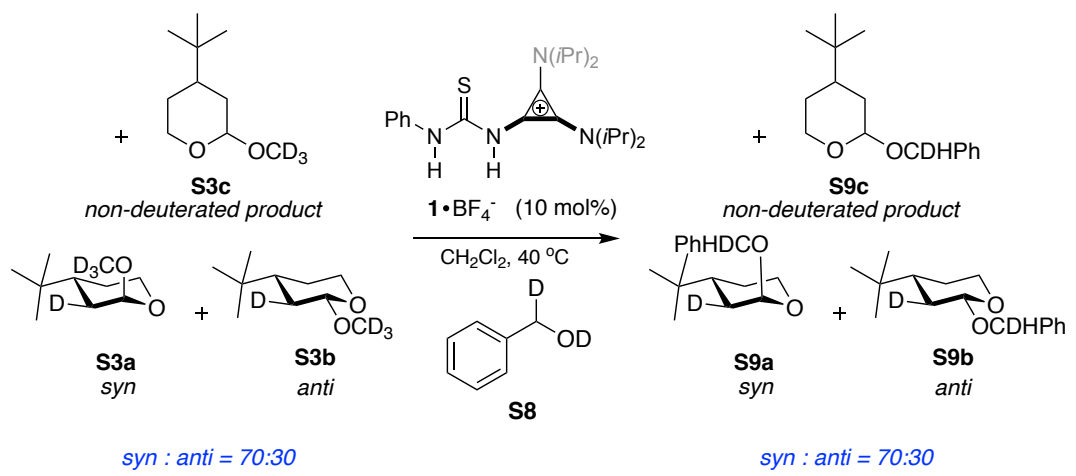


\section{Spectra}

1D NOESY NMR Spectrum of Thiourea $1 \cdot \mathrm{BF}_{4}^{-}{ }^{-}$with Irradiation at the Methine Signal (600

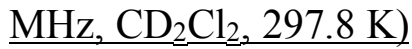

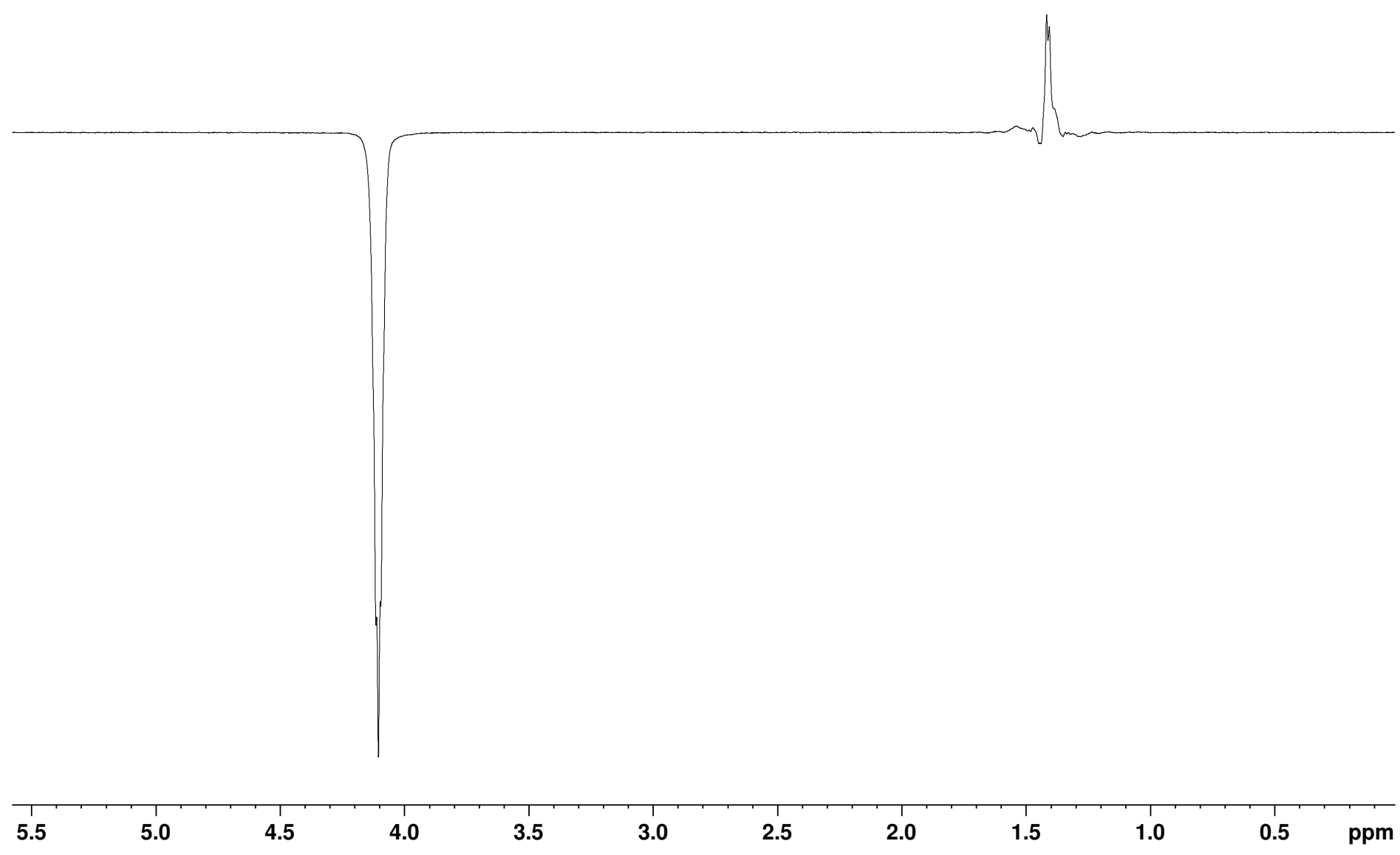


1D NOESY NMR Spectrum of Thiourea $1 \cdot \mathrm{BF}_{4}^{-}$with Irradiation at the N(3)-H Signal (600 $\left.\underline{\mathrm{MHz}, \mathrm{CD}_{2}} \underline{\mathrm{Cl}}_{2}, 297.8 \mathrm{~K}\right)$

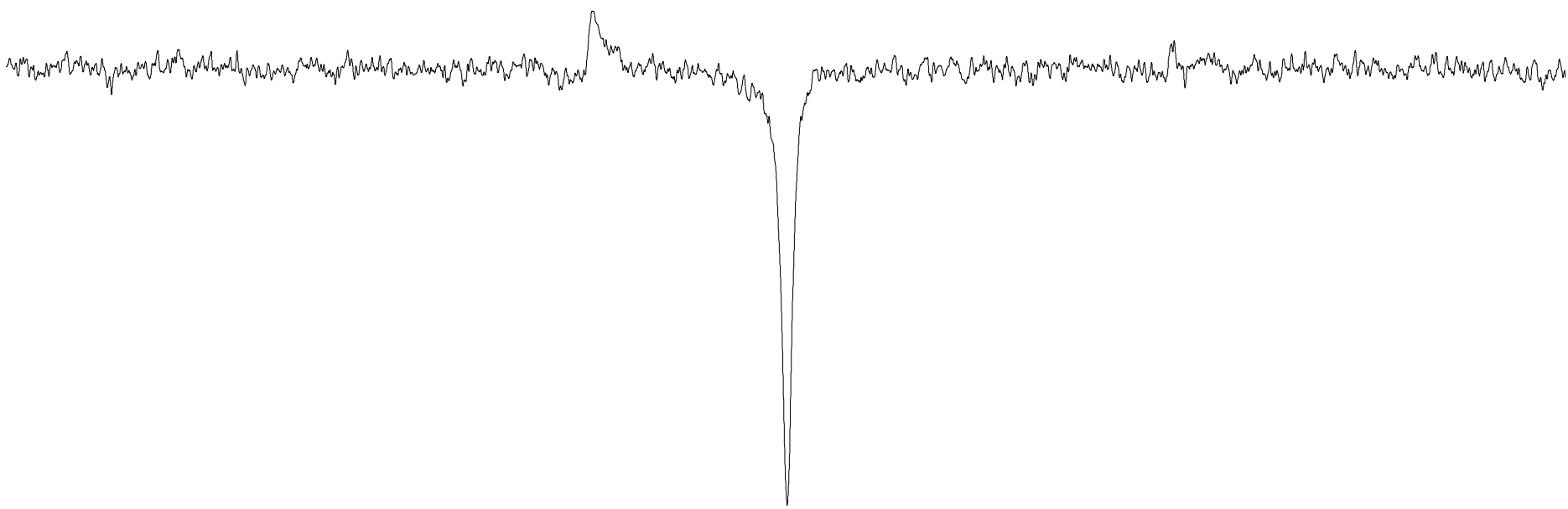

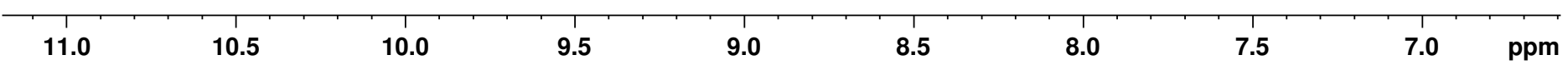


1D NOESY NMR Spectrum of Thiourea 1•BF 4 - with Irradiation at the N(4)-H Signal (600 $\underline{\mathrm{MHz}, \mathrm{CD}_{2}} \underline{\mathrm{Cl}}_{2}, 297.8 \mathrm{~K}$ )

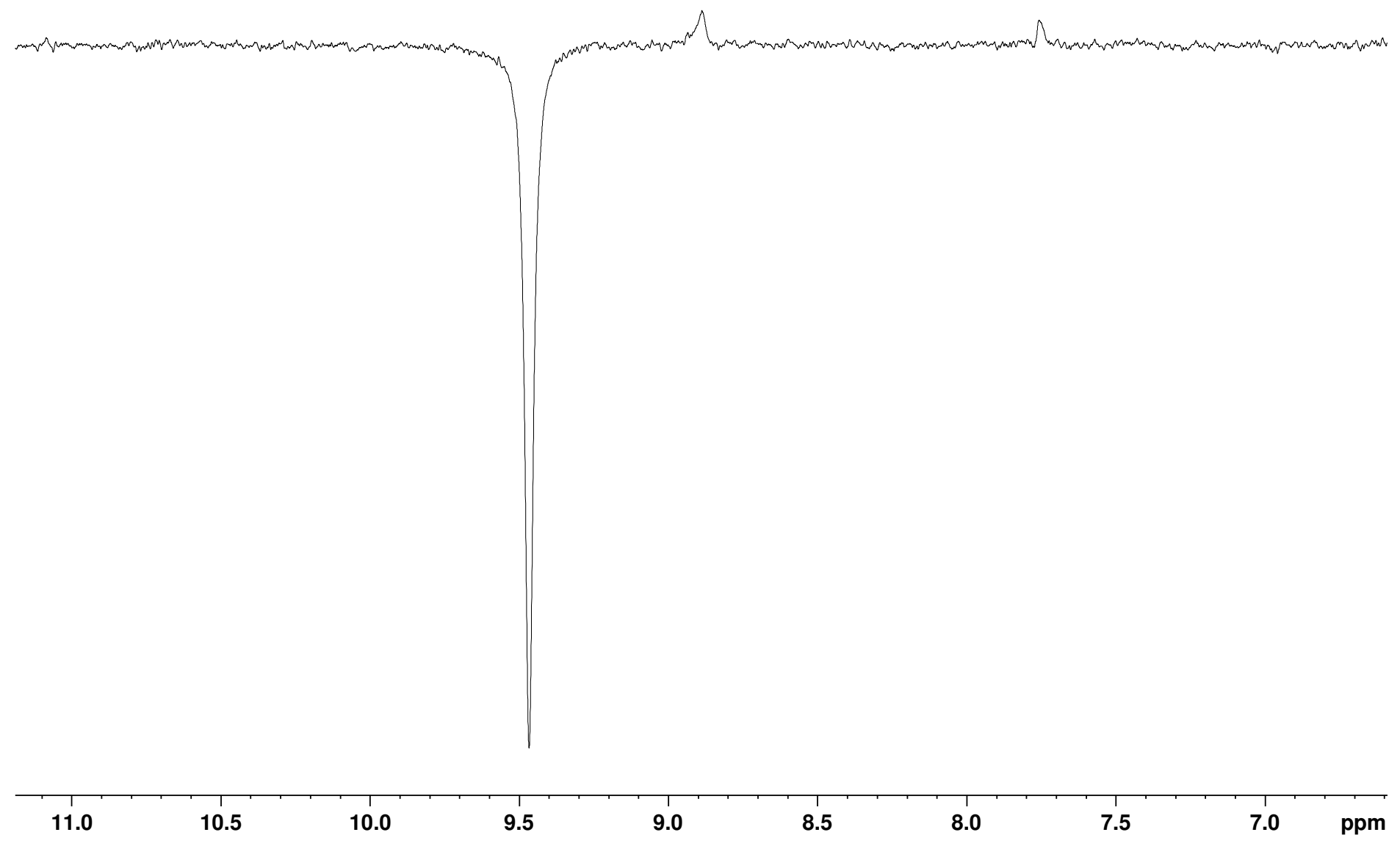


1D NOESY NMR Spectrum of Thiourea $1 \cdot \mathrm{BF}_{4}^{-}$with Irradiation at the N(4)-H Signal (600 $\left.\underline{\mathrm{MHz}, \mathrm{CD}_{2}} \underline{\mathrm{Cl}}_{2}, 193.0 \mathrm{~K}\right)$

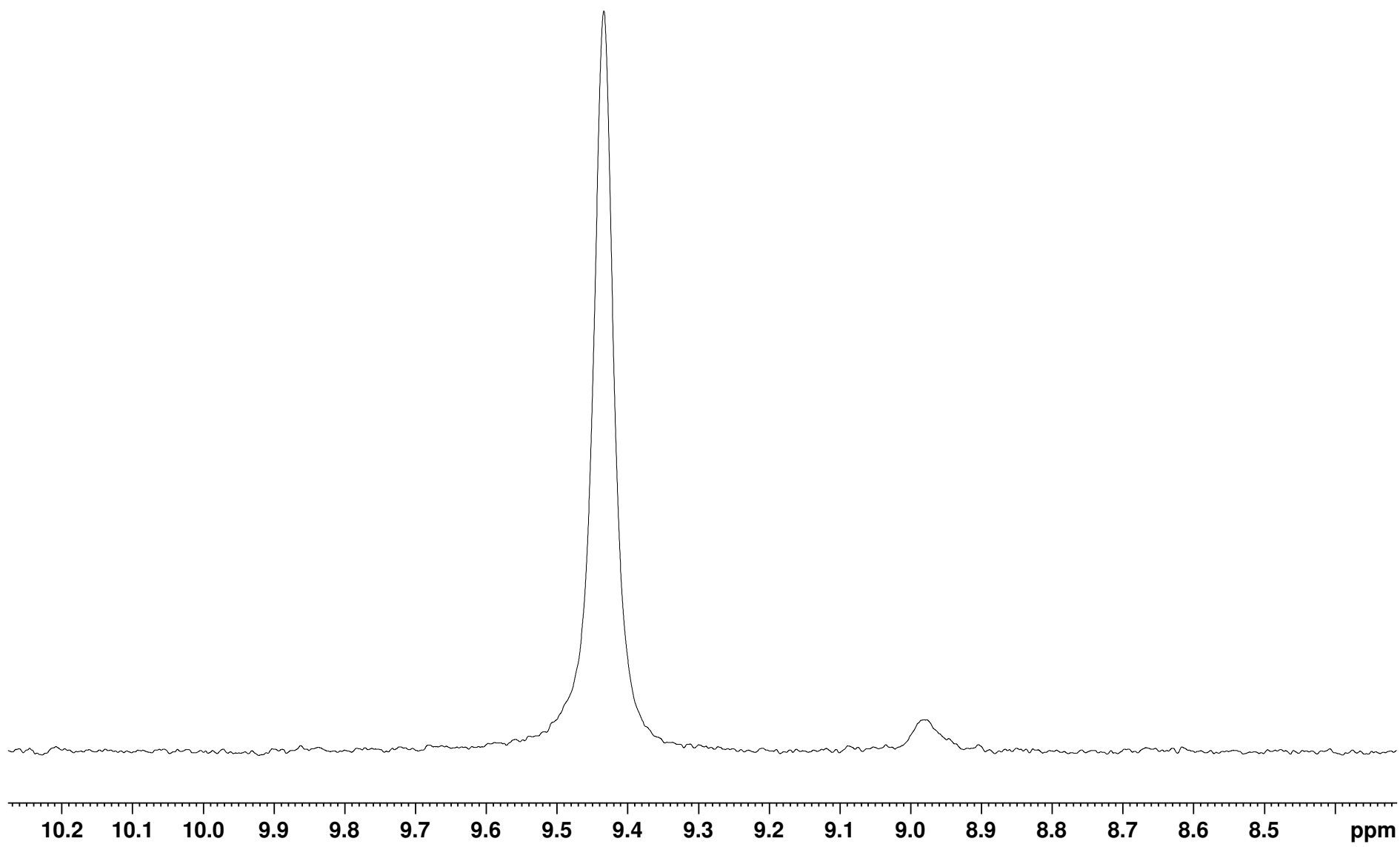


${ }^{1}$ H NMR Spectrum of Deuterated Mixture - syn and anti Product Isomers (S3a-c)

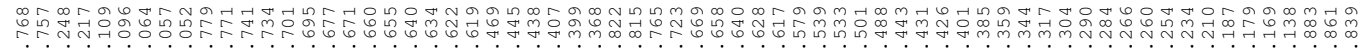

(n)

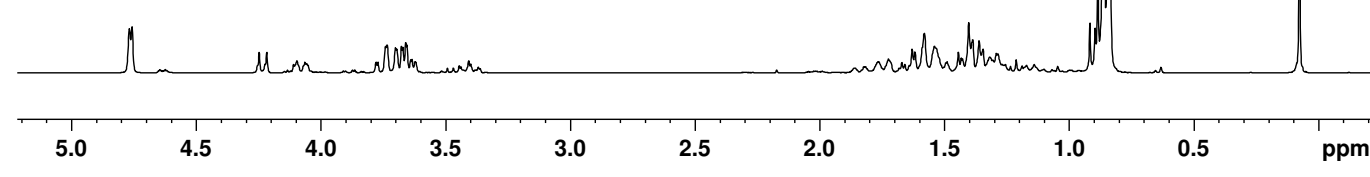

${ }^{13} \mathrm{C}$ NMR Spectrum of a Deuterated Mixture - syn and anti Product Isomers (S3a-c)

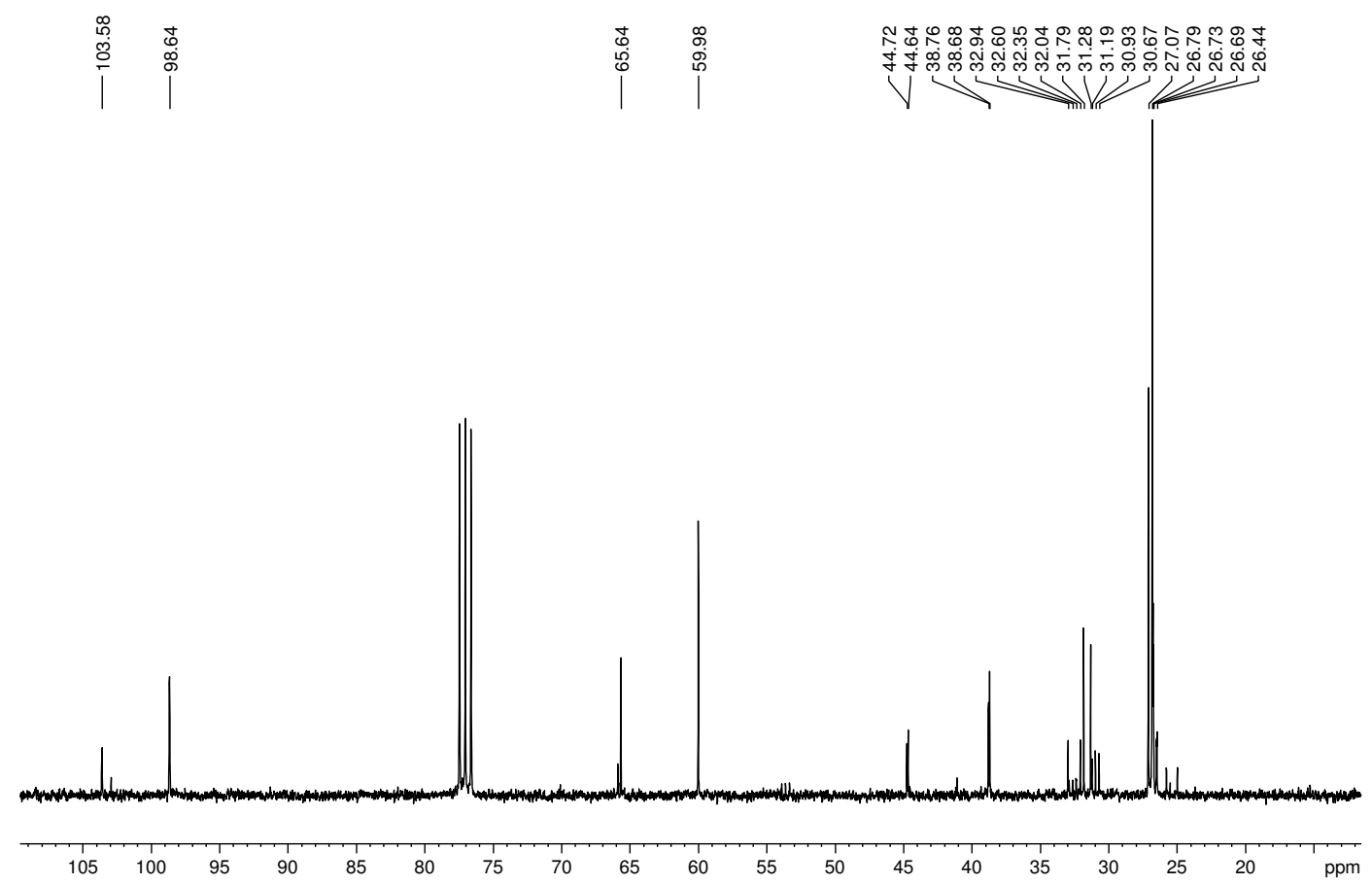




\section{$\underline{{ }^{1} \mathrm{H} \text { NMR Spectrum of } \mathbf{S 4}}$}

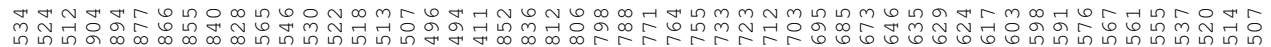

$\mathrm{H}_{3} \mathrm{C}$-OTHP

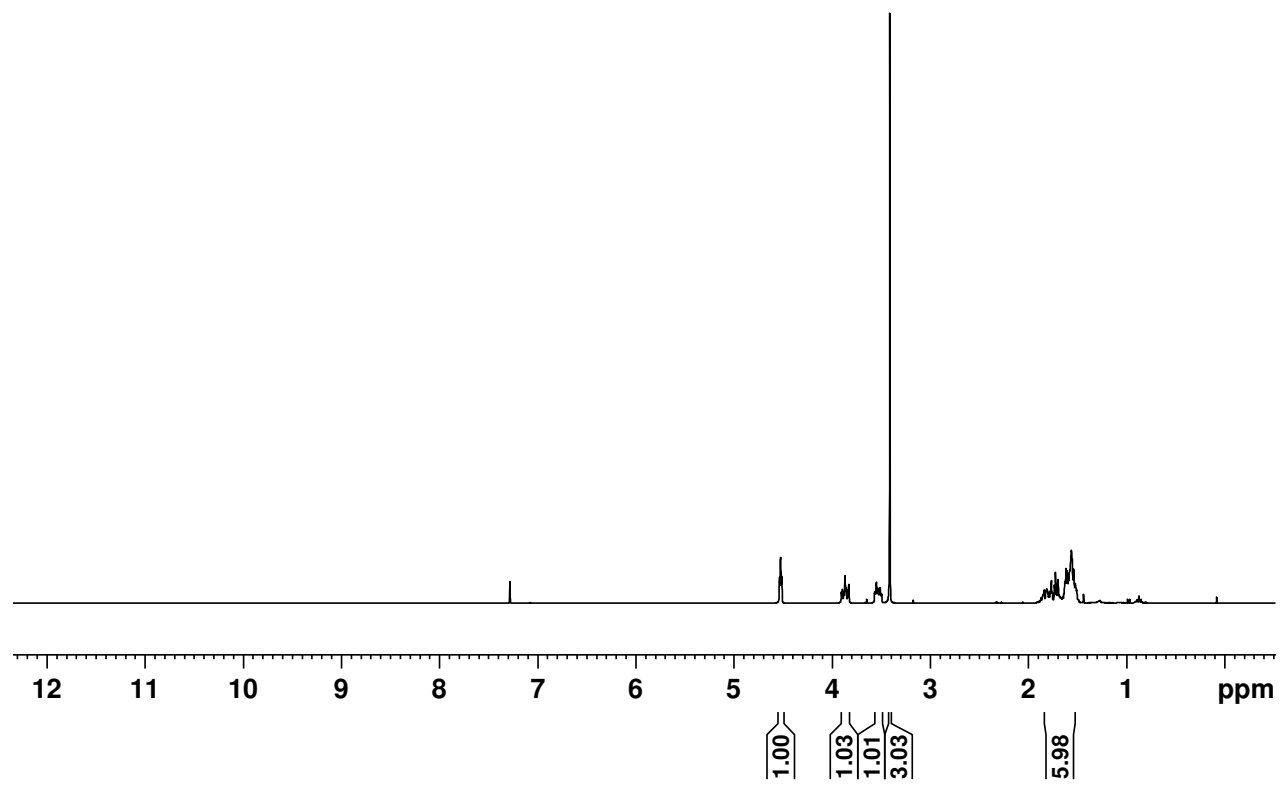

${ }^{1} \mathrm{H}$ NMR Spectrum of S4 and S5 (3:1)
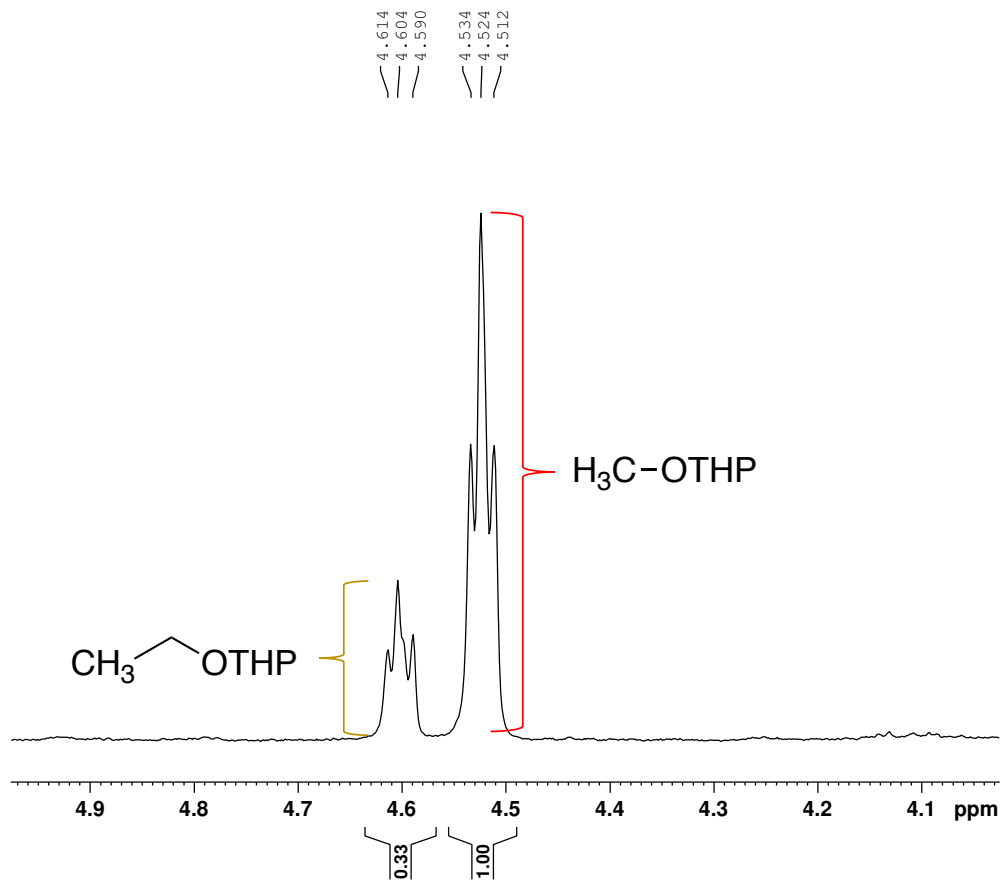
${ }^{1} \mathrm{H}$ NMR Spectrum of S4 (Minor), S6, and S7 (Major)

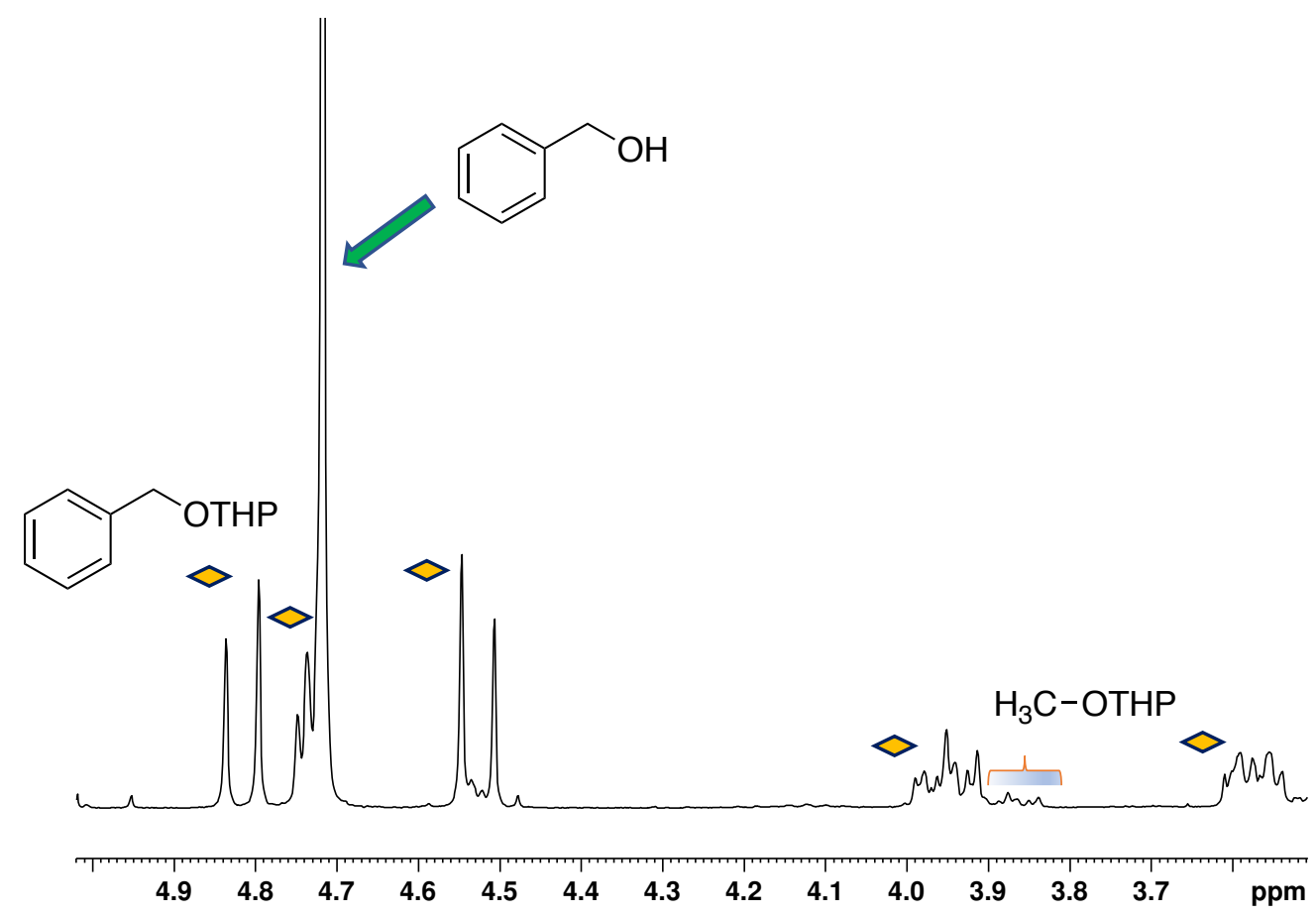

${ }^{1} \mathrm{H}$ NMR Spectrum of Deuterated Mixture - syn and anti Product Isomers (S9a-c)

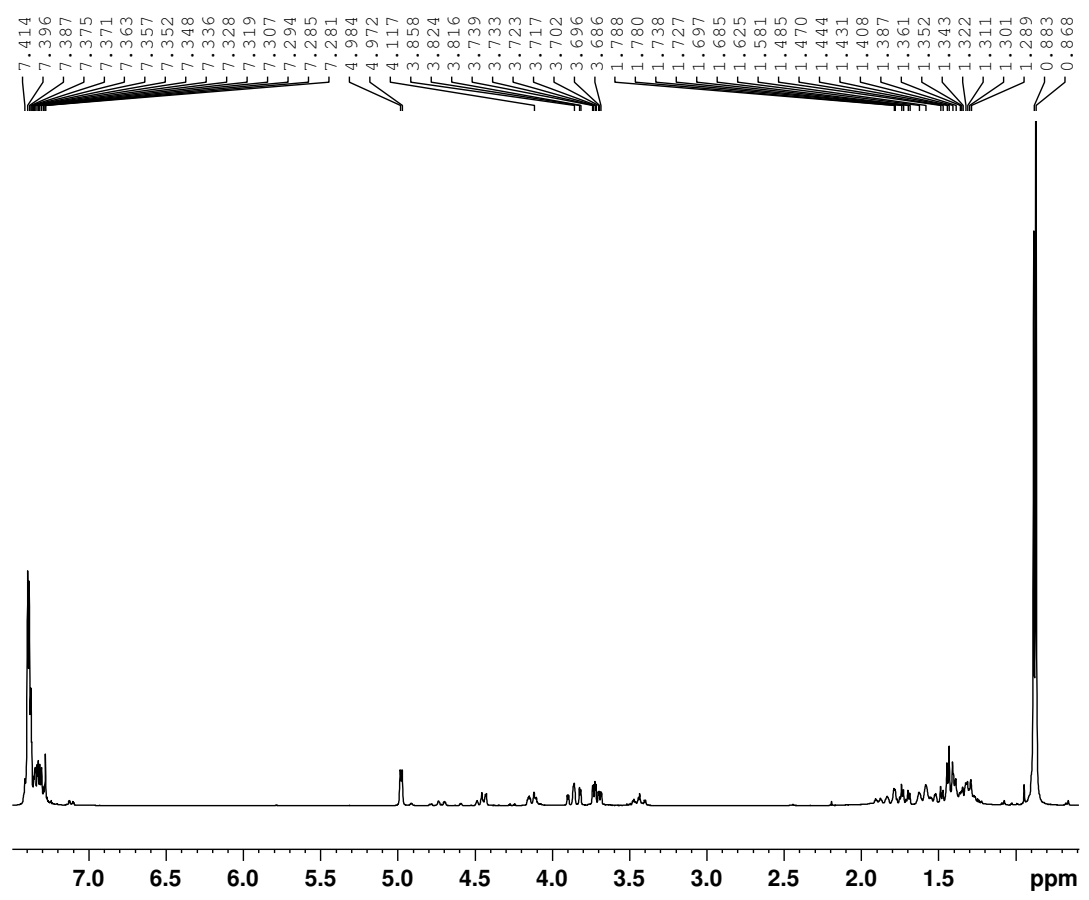


${ }^{13} \mathrm{C}$ NMR Spectrum of Deuterated Mixture - syn and anti Product Isomers (S9a-c)
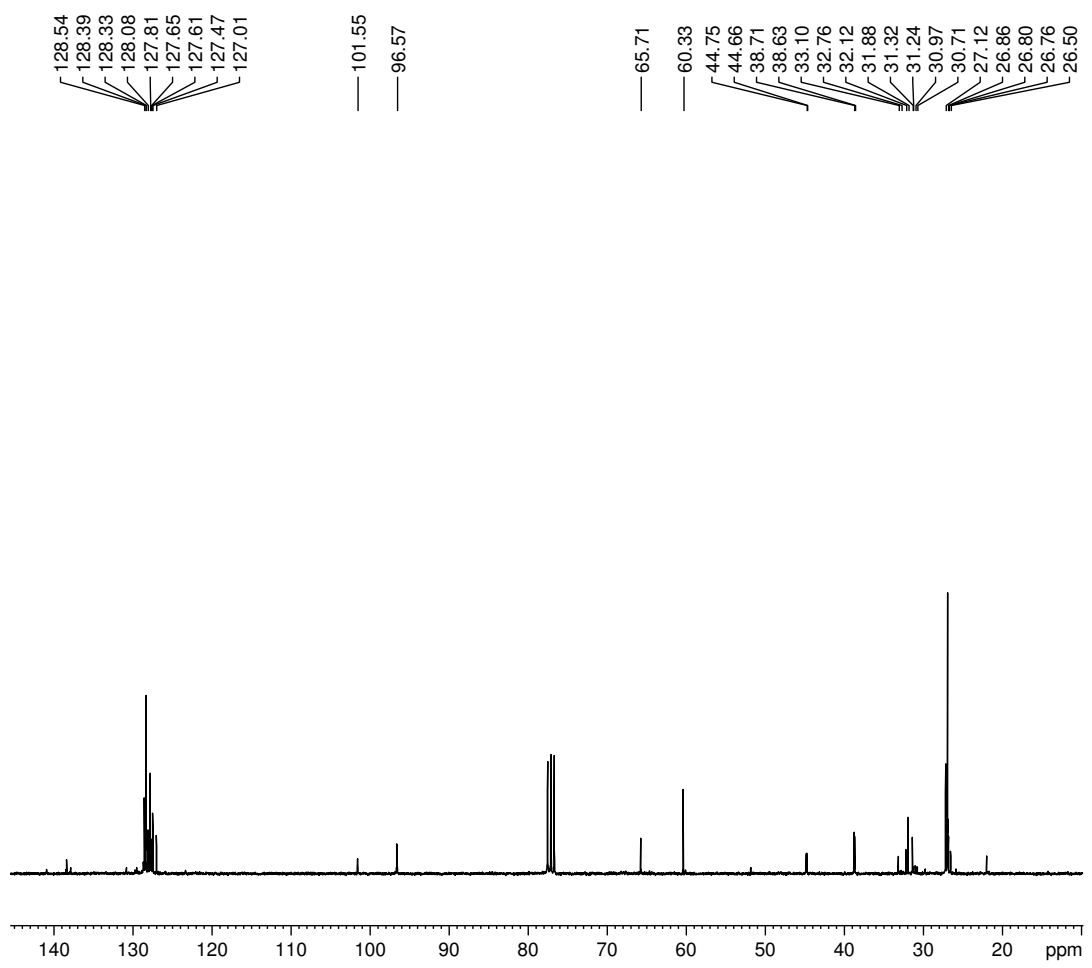

DEPT-135 NMR Spectrum of Deuterated Mixture - syn and anti Product Isomers (S9a-c)

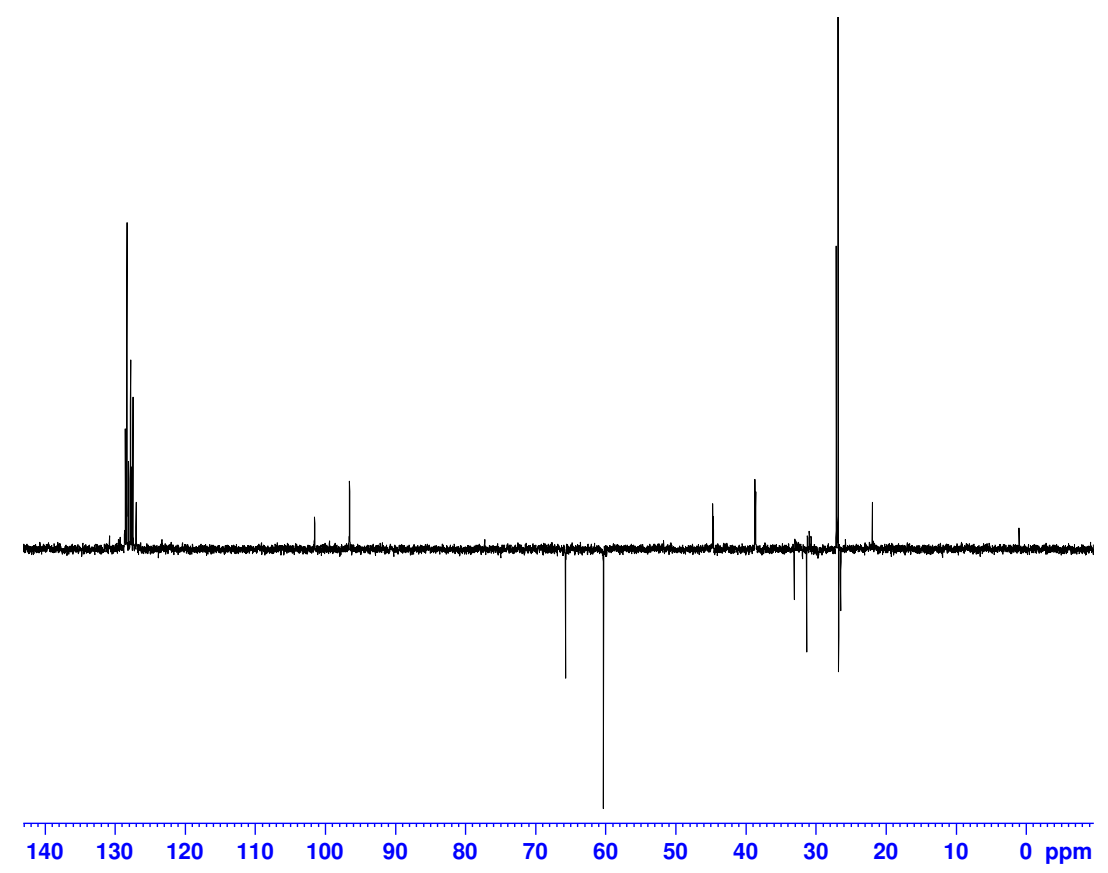


2D HSQC NMR Spectrum of Deuterated Mixture - syn and anti Product Isomers (S9a-c)

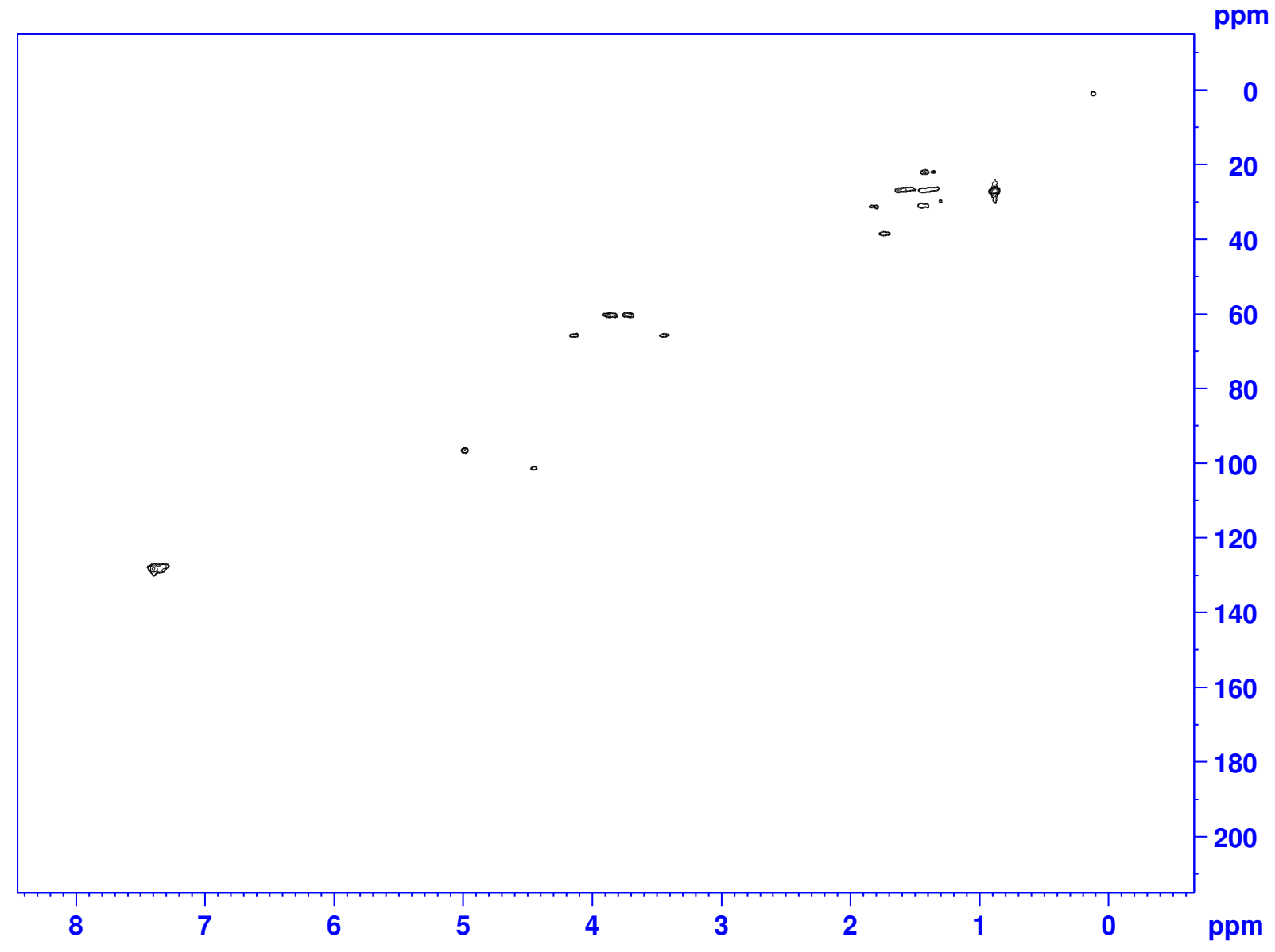


2D COSY NMR Spectrum of Deuterated Mixture - syn and anti Product Isomers (S9a-c)

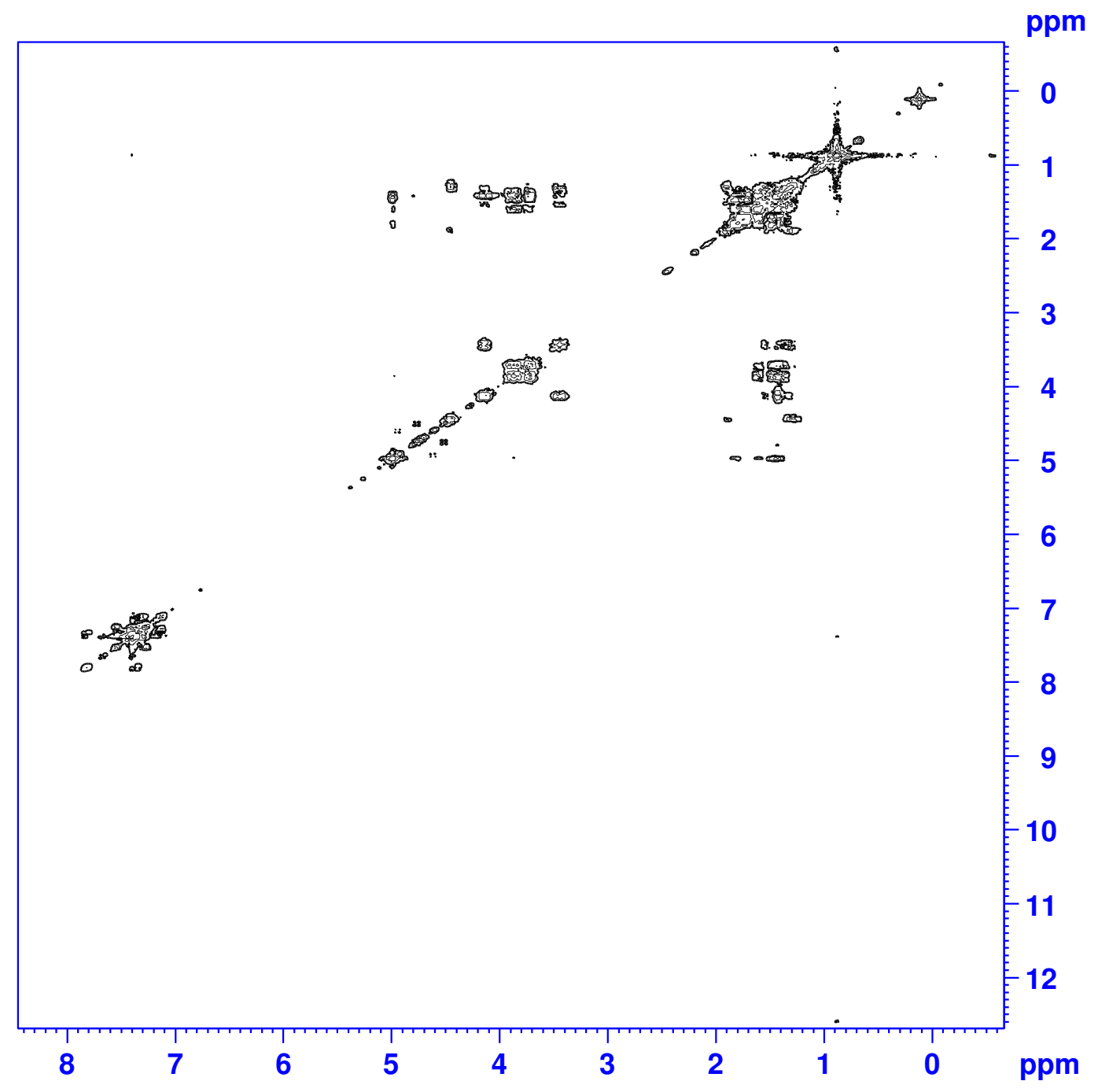




\section{D COSY NMR Spectrum of $\mathbf{S 7}$}

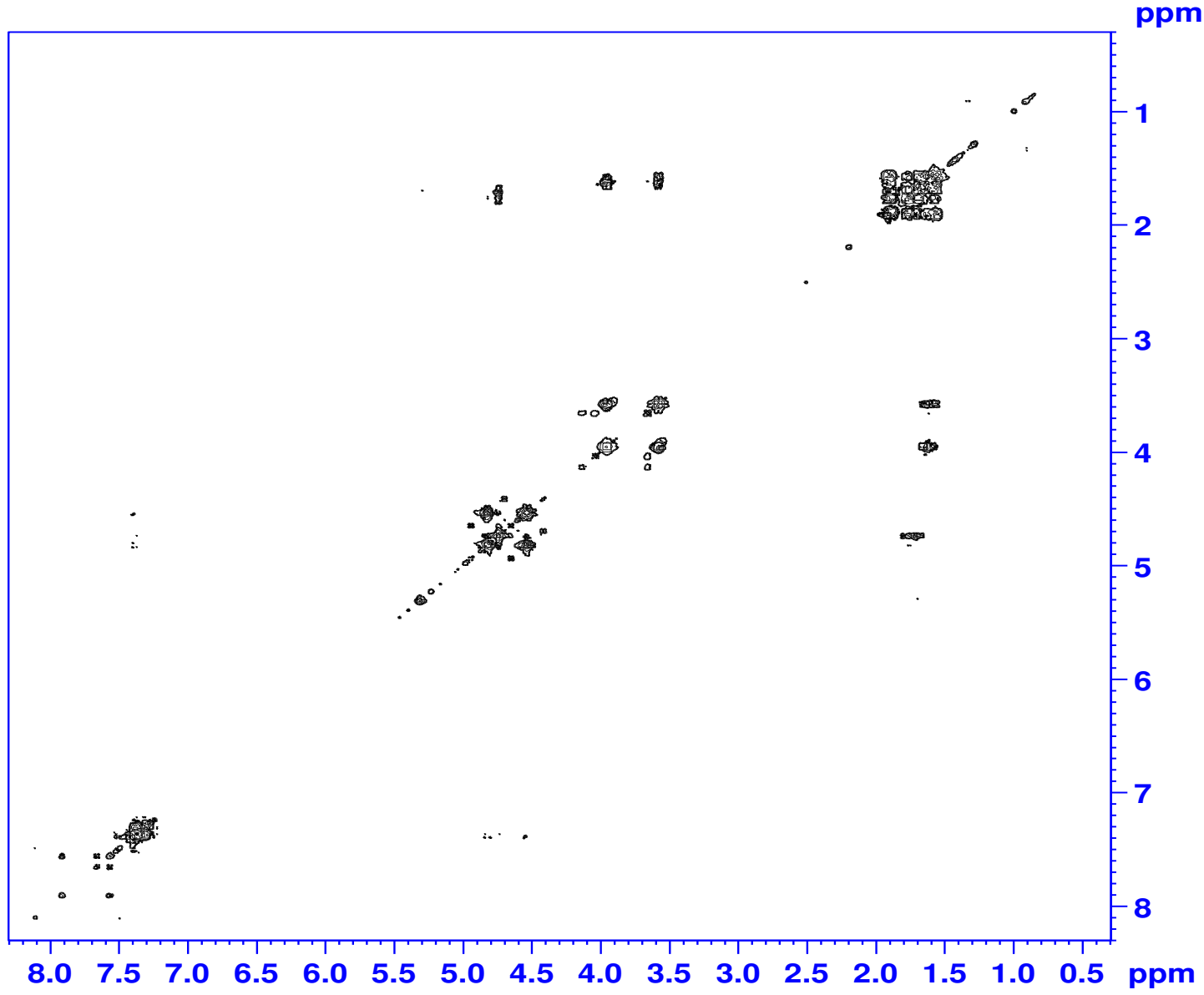




\section{D HSQC NMR Spectrum S7}

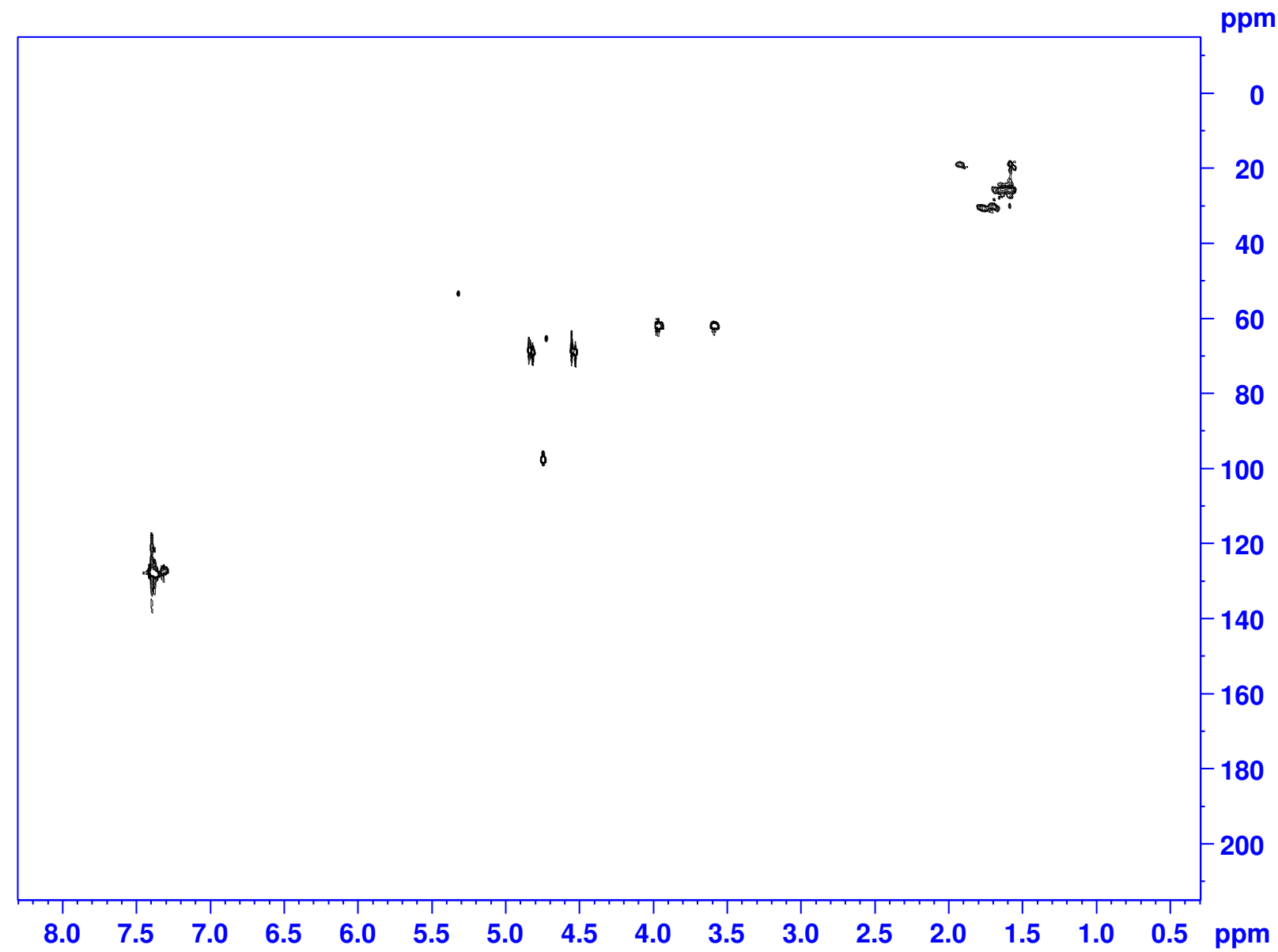


DEPT-55 NMR Spectrum of $\mathbf{S 7}(100 \%$ conversion to product)
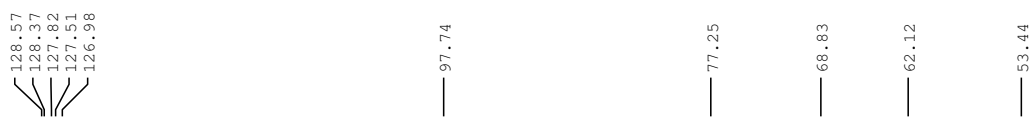

Integral 4

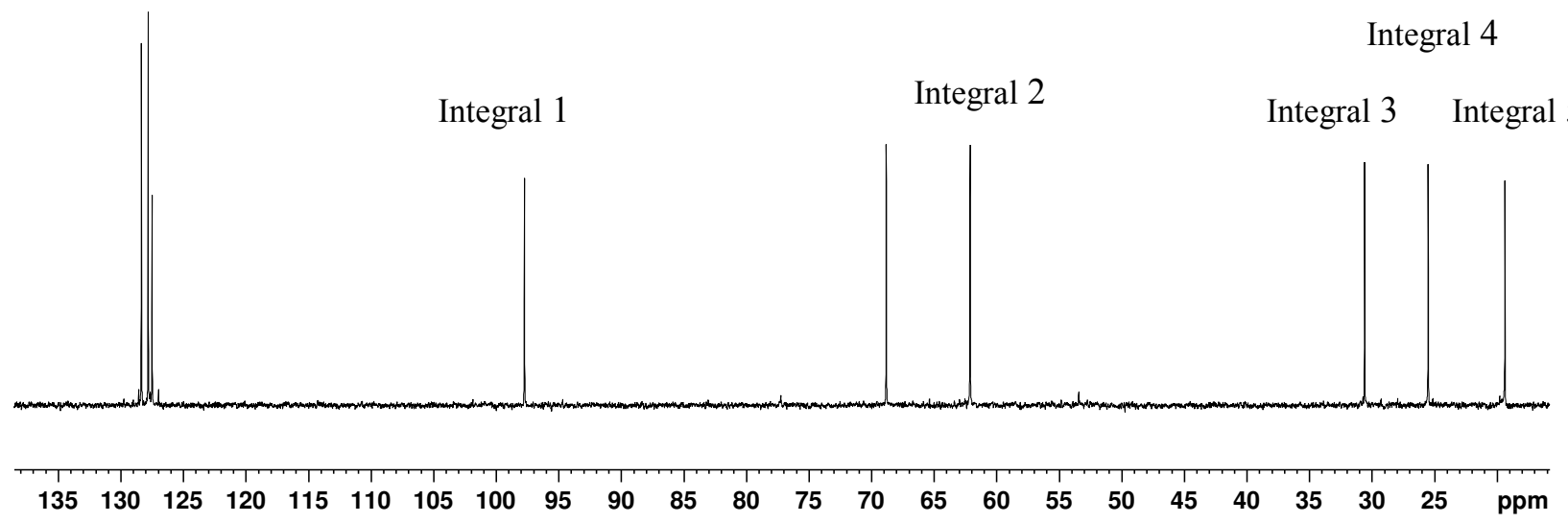

Table S5. Integration values denoted for the peaks of interest at 100\% conversion.

\begin{tabular}{|l|r|r|r|r|r|r|}
\hline Object & Integral [abs] & Integral [rel] & Peaks & Range (F1) from & Range (F1) to & (F1) [ppm] \\
\hline Integral 1 & 413486340.5 & 0.7528 & 1 & 97.892 & 97.591 & 97.7418 \\
\hline Integral 2 & 549266376.3 & 1 & 1 & 62.316 & 61.931 & 62.1233 \\
\hline Integral 3 & 439738826.3 & 0.8006 & 1 & 30.74 & 30.45 & 30.5949 \\
\hline Integral 4 & 461883042.8 & 0.8409 & 1 & 25.868 & 25.272 & 25.5701 \\
\hline Integral 5 & 481648078.3 & 0.8769 & 1 & 19.68 & 19.09 & 19.3847 \\
\hline
\end{tabular}




\section{DEPT-55 NMR Spectrum of S7 ( $2 \%$ conversion to product)}
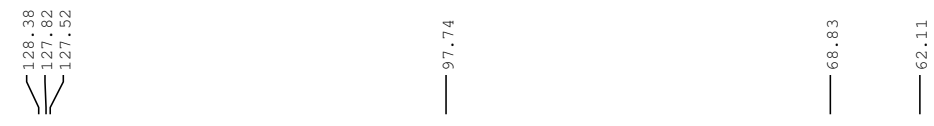

Integral 4

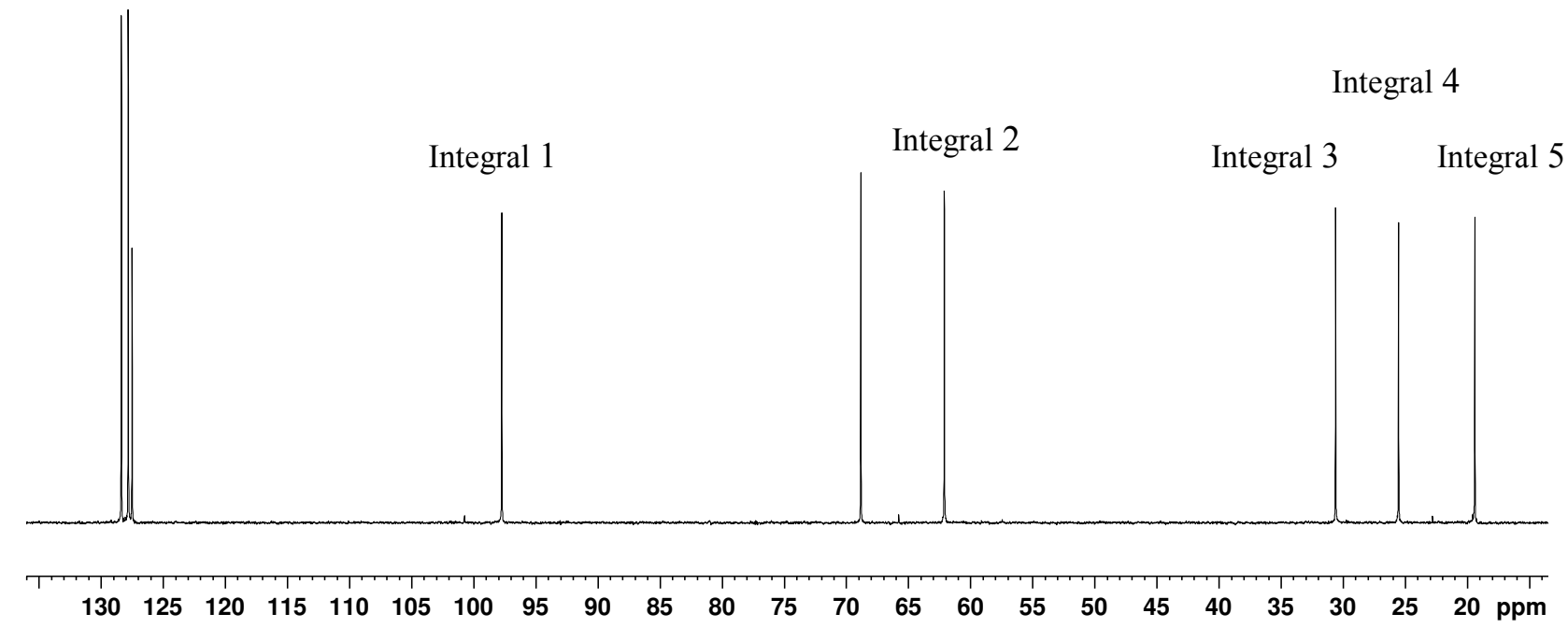

Table S6. Integration values denoted for the peaks of interest at $2 \%$ conversion.

\begin{tabular}{|l|r|r|r|r|r|r|}
\hline Object & \multicolumn{1}{|c|}{ Integral [abs] } & Integral [rel] & Peaks & Range (F1) from & Range (F1) to & (F1) [ppm] \\
\hline Integral 1 & 1450629516 & 0.8404 & 1 & 97.733 & 97.226 & 97.7556 \\
\hline Integral 2 & 1726021660 & 1 & 1 & 62.503 & 61.709 & 62.1059 \\
\hline Integral 3 & 1539357156 & 0.8919 & 1 & 30.954 & 30.16 & 30.5569 \\
\hline Integral 4 & 1452714348 & 0.8417 & 1 & 25.815 & 25.213 & 25.5137 \\
\hline Integral 5 & 1504962524 & 0.8719 & 1 & 19.74 & 19.07 & 19.405 \\
\hline
\end{tabular}




\section{$\underline{{ }^{1} \mathrm{H}}$ NMR Spectrum of $\mathbf{3}$}
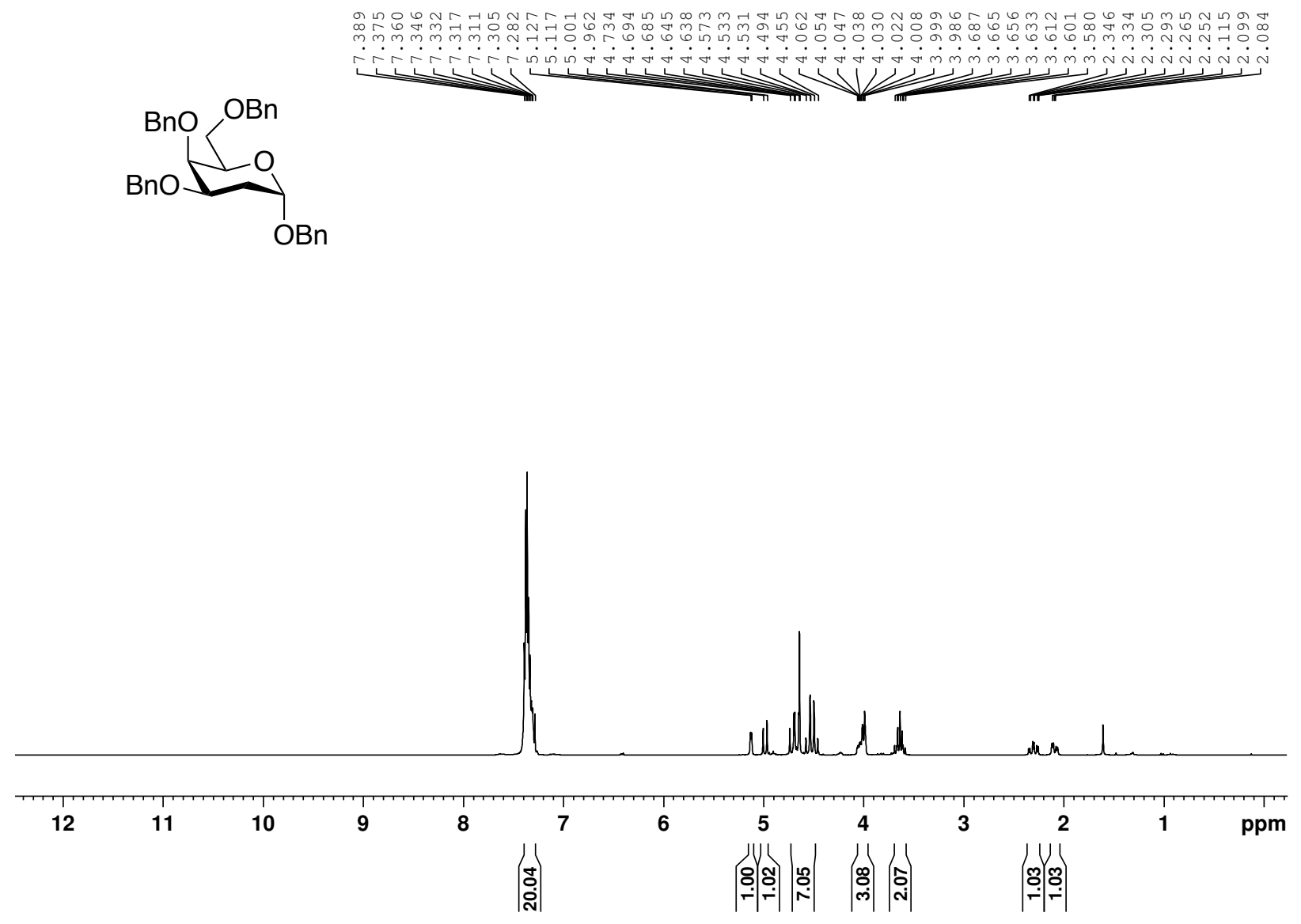


\section{References}

(1) Frisch, M. J.; Trucks, G. W.; Schlegel, H. B.; Scuseria, G. E.; Robb, M. A.; Cheeseman, J. R.; Scalmani, G.; Barone, V.; Mennucci, B.; Petersson, G. A.; Nakatsuji, H.; Caricato, M.; Li, X.; Hratchian, H. P.; Izmaylov, A. F.; Bloino, J.; Zhang, G.; Sonnenberg, J. L.; Hada, M.; Ehara, M.; Toyota, K.; Fukuda, R.; Hasegawa, J.; Ishida, M.; Nakajima, T.; Honda, Y.; Kitao, O.; Nakai, H.; Vreven, T.; Montgomery, J. A.; Peralta, Jr., J. E.; Ogliaro, F.; Bearpark, M.; Heyd, J. J.; Brothers, E.; Kudin, K. N.; Staroverov, V. N.; Kobayashi, R.; Normand, J.; Raghavachari, K.; Rendell, A.; Burant, J. C.; Iyengar, S. S.; Tomasi, J.; Cossi, M.; Rega, N.; Millam, J. M.; Klene, M.; Knox, J.e.; Cross, J. B.; Bakken, V.; Adamo, C.; Jaramillo, J.; Gomperts, R.; Stratmann, R. E.; Yazyev, O.; Austin, A. J.; Cammi, A. R.; Pomelli, C.; Ochterski, J. W.; Martin, R. L.; Morokuma, K.; Zakrzewski, V. G.; Voth, G. A.; Salvador, P.; Dannenberg, J. J.; Dapprich, S.; Daniels, A. D.; Farkas, Ö.; Foresman, J. B.; Ortiz, J. V.; Cioslowski, J.; Fox, D. J. Gaussian 09, Revision D.02; Gaussian, Inc., Wallingford, CT, 2009.

(2) Chai, J.-D.; Head-Gordon, M. Long-range corrected hybrid density functionals with damped atom-atom dispersion corrections. Phys. Chem. Chem. Phys., 2008, 10, 6615-6620.

(3) Krishnan, R.; Binkley, J. S.; Seeger, R.; Pople, J. A. Self-consistent molecular orbital methods. XX. A basis set for correlated wave functions. J. Chem. Phys., 1980, 72, 650-654.

(4) Scott, A. P.; Radom, L. Harmonic Vibrational Frequencies: An Evaluation of Hartree-Fock, Møller-Plesset, Quadratic Configuration Interaction, Density Functional Theory, and Semiempirical Scale Factors. J. Phys. Chem., 1996, 100, 16502-16513.

(5) Peng, C. Y.; Ayala, P. Y.; Schlegel, H. B.; Frisch, M. J. Using redundant internal coordinates to optimize equilibrium geometries and transition states. J. Comp. Chem., 1996, 17, 49-56.

(6) Hratchian, H. P.; Schlegel, H. B Using Hessian Updating To Increase the Efficiency of a Hessian Based Predictor-Corrector Reaction Path Following Method. J. Chem. Theory Comput., 2005, 1, 61-69.

(7) Hratchian, H. P.; Schlegel, H. B. Accurate reaction paths using a Hessian based predictorcorrector integrator. J. Chem. Phys., 2004, 120, 9918-9924.

(8) Marenich, A. V.; Cramer, C. J.; Truhlar, D. G. Universal solvation model based on solute electron density and on a continuum model of the solvent defined by the bulk dielectric constant and atomic surface tensions. J. Phys. Chem. B, 2009, 113, 6378-6396.

(9) Tomasi, J.; Mennucci, B.; Cammi, R. Quantum Mechanical Continuum Solvation Models. Chem. Rev., 2005, 105, 2999-3094.

(10) C.Y. Legault. CYLview. Universite de Sherbrooke, Quebec, Canada, 2009, version 1.0b, http://www.cylview.org

(11) Dennington, R.; Keith, T.; Millam, J. GaussView. Semichem Inc., Shawnee Mission, KS, 2009, Version 5.

(12) Storer, J. W.; Giesen, D. J.; Cramer, C. J.; Truhlar, D. G., Class IV charge models: a new semiempirical approach in quantum chemistry. J. Comput. Aided. Mol. Des., 1995, 9, 87-110.

(13) Schrödinger Release 2019-2: MacroModel, Schrödinger, LLC, New York, NY, 2019.

(14) Smajlagic, I.; Durán, R.; Pilkington, M.; Dudding, T. Cyclopropenium Enhanced Thiourea Catalysis. J. Org. Chem. 2018, 83, 13973-13980.

(15) Wheeler, S. E.; Seguin, T. J.; Guan, Y.; Doney, A. C. Noncovalent Interactions in Organocatalysis and the Prospect of Computational Catalyst Design. Acc. Chem. Res. 2016, 49, 1061-1069. 
(16) Kennedy, C. R.; Lin, S.; Jacobsen, E. N. The Cation- $\pi$ Interaction in Small-Molecule Catalysis. Angew. Chem. Int. Ed. 2016, 55, 12596-12624. 